Florida International University

FIU Digital Commons

FIU Electronic Theses and Dissertations

University Graduate School

$1-9-2020$

\title{
Towards UAV Assisted 5G Public Safety Network
}

Abhaykumar Kumbhar

Florida International University, akumb004@fiu.edu

Follow this and additional works at: https://digitalcommons.fiu.edu/etd

Part of the Systems and Communications Commons

\section{Recommended Citation}

Kumbhar, Abhaykumar, "Towards UAV Assisted 5G Public Safety Network" (2020). FIU Electronic Theses and Dissertations. 4448.

https://digitalcommons.fiu.edu/etd/4448

This work is brought to you for free and open access by the University Graduate School at FIU Digital Commons. It has been accepted for inclusion in FIU Electronic Theses and Dissertations by an authorized administrator of FIU Digital Commons. For more information, please contact dcc@fiu.edu. 


\section{FLORIDA INTERNATIONAL UNIVERSITY \\ Miami, Florida}

TOWARDS UAV ASSISTED 5G PUBLIC SAFETY NETWORK

A dissertation submitted in partial fulfillment of the

requirements for the degree of DOCTOR OF PHILOSOPHY

in

ELECTRICAL ENGINEERING

by

Abhaykumar Kumbhar 
To: Dean John L. Volakis

College of Engineering and Computing

This dissertation, written by Abhaykumar Kumbhar, and entitled Towards UAV Assisted 5G Public Safety Network, having been approved in respect to style and intellectual content, is referred to you for judgment.

We have read this dissertation and recommend that it be approved.

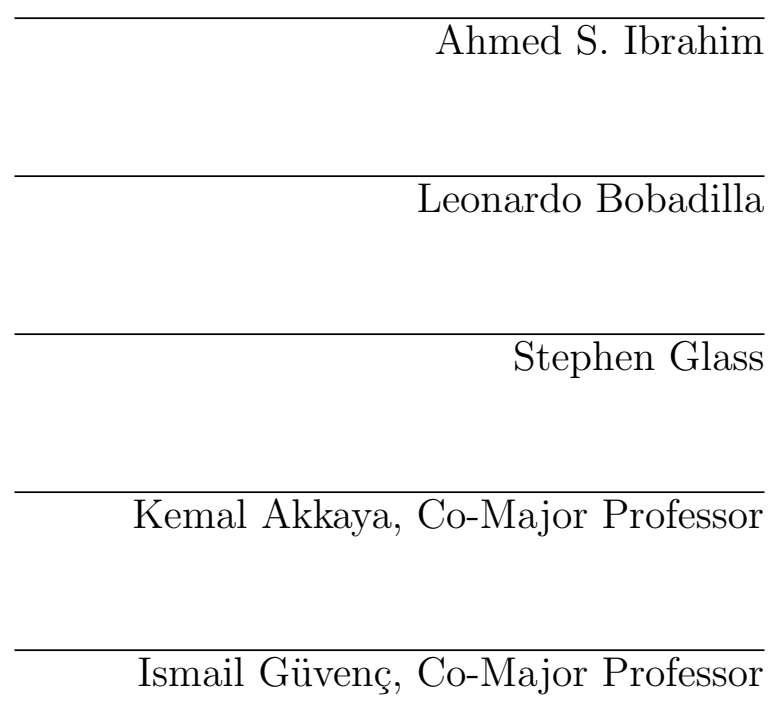

Date of Defense: January 9, 2020

The dissertation of Abhaykumar Kumbhar is approved.

Dean John L. Volakis
College of Engineering and Computing

Andrés G. Gil

Vice President for Research and Economic Development and Dean of the University Graduate School

Florida International University, 2020 
(C) Copyright 2020 by Abhaykumar Kumbhar All rights reserved. 


\section{DEDICATION}

I dedicate this dissertation to my parents, Pushpa Kumbhar and Madan Kumbhar and wife Shubhangi Kumbhar, who have been my support system. 


\section{ACKNOWLEDGMENTS}

Being competitive at Motorola Solutions and meeting the research expectation of the Ph.D. program at Florida International University was challenging and required lots of dedication, hard work, and perseverance.

The foremost, I would like to thank my advisor Dr. Ismail Güvenç. His help, support, encouragement, and guidance made this dissertation possible. He mentored me in the right direction during my research and has always been the guiding light. I have learned a lot from him; how to write technical papers with good flow, drafting your ideas, and mainly how to stay focused and dedicated. I would also like to thank and pay my gratitude to the members of my committee for their insightful comments, encouragement, and generous support.

I would also like to thank my managers and colleagues at Motorola Solutions facility in Plantation for being supportive and flexible with the work schedule. Special Thanks to Motorola Solutions. Inc for being generous and partially funding my Ph.D. at Florida International University.

I would like to thank my fellow lab mates at FIU MPACT for the stimulating discussions, and for all the fun we had. I am also thankful to my roommates and friends for their continuous support outside the university environment.

The last but not the least, I want to express my appreciation towards my parents for all the sacrifices they made for bringing me to this point. Finally, my deepest gratitude goes to my wife for being patient and sharing the joys and disappointments. 


\author{
ABSTRACT OF THE DISSERTATION \\ TOWARDS UAV ASSISTED 5G PUBLIC SAFETY NETWORK \\ by \\ Abhaykumar Kumbhar \\ Florida International University, 2020 \\ Miami, Florida \\ Professor Ismail Güvenç, Co-Major Professor \\ Professor Kemal Akkaya, Co-Major Professor
}

Ensuring ubiquitous mission-critical public safety communications (PSC) to all the first responders in the public safety network is crucial at an emergency site. The first responders heavily rely on mission-critical PSC to save lives, property, and national infrastructure during a natural or human-made emergency. The recent advancements in LTE/LTE-Advanced/5G mobile technologies supported by unmanned aerial vehicles (UAV) have great potential to revolutionize PSC.

However, limited spectrum allocation for LTE-based PSC demands improved channel capacity and spectral efficiency. An additional challenge in designing an LTE-based PSC network is achieving at least 95\% coverage of the geographical area and human population with broadband rates. The coverage requirement and efficient spectrum use in the PSC network can be realized through the dense deployment of small cells (both terrestrial and aerial). However, there are several challenges with the dense deployment of small cells in an air-ground heterogeneous network (AG-HetNet). The main challenges which are addressed in this research work are integrating UAVs as both aerial user and aerial base-stations, mitigating inter-cell interference, capacity and coverage enhancements, and optimizing deployment locations of aerial base-stations. 
First, LTE signals were investigated using NS-3 simulation and software-defined radio experiment to gain knowledge on the quality of service experienced by the user equipment (UE). Using this understanding, a two-tier LTE-Advanced AG-HetNet with macro base-stations and unmanned aerial base-stations (UABS) is designed, while considering time-domain inter-cell interference coordination techniques. We maximize the capacity of this AG-HetNet in case of a damaged PSC infrastructure by jointly optimizing the inter-cell interference parameters and UABS locations using a meta-heuristic genetic algorithm (GA) and the brute-force technique. Finally, considering the latest specifications in 3GPP, a more realistic three-tier LTE-Advanced AG-HetNet is proposed with macro base-stations, pico base-stations, and ground UEs as terrestrial nodes and UABS and aerial UEs as aerial nodes. Using metaheuristic techniques such as GA and elitist harmony search algorithm based on the GA, the critical network elements such as energy efficiency, inter-cell interference parameters, and UABS locations are all jointly optimized to maximize the capacity and coverage of the AG-HetNet. 


\section{TABLE OF CONTENTS}

CHAPTER

PAGE

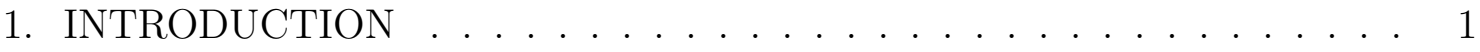

1.1 Public Safety Network . . . . . . . . . . . . . . . . . . . . 1

1.1 .1 Contributions . . . . . . . . . . . . . . . . . 2

1.1 .2 Publications . . . . . . . . . . . . . . . . . 3

1.2 Role of UAVs in Public Safety HetNets . . . . . . . . . . . . . . . 3

1.2 .1 Contributions . . . . . . . . . . . . . . . . 4

1.2 .2 Publications . . . . . . . . . . . . . . . . . . 6

1.3 Capacity and Coverage Enhancement . . . . . . . . . . . . 6

1.3 .1 Contributions . . . . . . . . . . . . . . . . . . 7

1.3 .2 Publications . . . . . . . . . . . . . . . . . . . 7

1.4 Joint Optimization of UABS Locations and ICIC Parameters . . . . . . . 8

1.4 .1 Contributions . . . . . . . . . . . . . . . . . . 8

1.4 .2 Publications . . . . . . . . . . . . . . . . . . . . 9

2. LITERATURE REVIEW . . . . . . . . . . . . . . . . . . . . 10

2.1 Public Safety Networks . . . . . . . . . . . . . . . . . . . . . . 10

2.2 Interference Coordination and Capacity Analysis of two-tier AG-HetNet 10

2.3 Heuristics Approach used in Joint Optimization of Interference Coordination and UABS Locations in AG-HetNet . . . . . . . . . . . . 12

2.4 Interference Coordination and Capacity and Coverage Analysis of threetier AG-HetNet . . . . . . . . . . . . . . . . . . . 13

3. PUBLIC SAFETY NETWORKS . . . . . . . . . . . . . . . . . . . . . 19

3.1 Introduction . . . . . . . . . . . . . . . . . . . . . . . . . . . . 19

3.2 LMRS Network . . . . . . . . . . . . . . . . . . . . . . 20

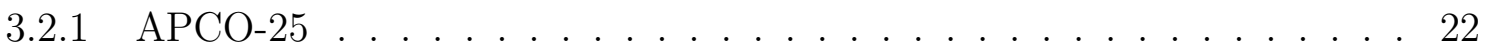

3.2 .2 TETRA . . . . . . . . . . . . . . . . . . 23

3.3 LTE Broadband Network . . . . . . . . . . . . . . . . . . . . . . 25

3.4 Major Challenges in PSNs . . . . . . . . . . . . . . . . . 26

3.4 .1 Network Congestion . . . . . . . . . . . . . . . . . . . . 26

3.4.2 Maintaining Ubiquitous Throughput and Connectivity . . . . . . . . 27

3.4 .3 Interoperability . . . . . . . . . . . . . . . . . . . . 28

3.5 Case Study: Public Safety Spectrum Allocation in the United States . . 29

3.5.1 $700 \mathrm{MHz}$ Public Safety Spectrum . . . . . . . . . . . . . . . . 29

3.5.2 $800 \mathrm{MHz}$ Public Safety Spectrum . . . . . . . . . . . . . . . . 32

3.5.3 $900 \mathrm{MHz}$ Public Safety Spectrum . . . . . . . . . . . . . . . . . 33

3.5.4 VHF and UHF Public Safety Spectrum . . . . . . . . . . . . . . 33

3.5.5 4.9 GHz Public Safety Spectrum _. . . . . . . . . . . . . . 34

3.6 Case Study: LTE-based FirstNet PSN _ . . . . . . . . . . . . . . 35

3.6 .1 FirstNet Architecture . . . . . . . . . . . . . . . . . 37 
3.7 LMRS and LTE Convergence . . . . . . . . . . . . . . . . . . . . . . 39

3.8 Mission-Critical PTT over LTE . . . . . . . . . . . . . . . . . . . . . 41

3.9 Comparison of LMRS and LTE PSC . . . . . . . . . . . . . . . 43

3.9.1 Network Architecture . . . . . . . . . . . . . . . . . . . . 43

3.9.2 Channel Configuration and Frame Structure . . . . . . . . . . . . . . 45

3.10 Emerging Technologies for PSC . . . . . . . . . . . . . . . 48

3.10 .1 Multimedia Broadcast Multicast Service (MBMS) . . . . . . . . . . . 49

3.10 .2 Millimeter Wave (mmWave) . . . . . . . . . . . . . . . . . . . 50

3.10 .3 Massive MIMO . . . . . . . . . . . . . . . . . . . . . . . . 51

3.10 .4 Small Cells . . . . . . . . . . . . . . . . . . . . . 52

3.10 .5 Unmanned Aerial Vehicles (UAVs) _ . . . . . . . . . . . . . 54

3.10 .6 LTE-based V2X Communication . . . . . . . . . . . . . . 55

3.10 .7 License Assisted Access (LAA) . . . . . . . . . . . . . . . 56

3.10 .8 Spectrum sharing and cognitive radio . . . . . . . . . . . . . 58

3.10 .9 Wireless Sensor Networks . . . . . . . . . . . . . . . . . . 59

3.10 .10 Internet of Things (IoT) . . . . . . . . . . . . . . . . . 60

3.10.11 Cybersecurity Enhancements and Data Analytics for PSNs . . . . . . . 61

3.11 Public safety broadband deployments in other regions of the world. . . . 63

3.12 Issues and Open Research Directions . . . . . . . . . . . . . . . 65

4. APCO-25 AND PUBLIC SAFETY LTE NS-3 SIMULATION . . . . . . 69

4.1 NS-3 Simulation of LTE Band Class 14 . . . . . . . . . . . . . . . . . 69

4.1.1 Scenario:Average Downlink and Uplink Throughput . . . . . . . . . . 70

4.1.2 Scenario: Aggregate throughput vs. Distance . . . . . . . . . . . . 71

4.1.3 Scenario: Signal quality measurement . . . . . . . . . . . . . . . 72

4.1.4 Scenario: Inter-cell interference measurement . . . . . . . . . . . . 73

4.2 NS-3 Simulation Setup for APCO-25 . . . . . . . . . . . . . . 75

4.2.1 Scenario: Throughput and SINR Simulation of APCO-25 Portable and

Mobile Devices . . . . . . . . . . . . . . . . . 77

4.3 NS-3 Simulation Result Analysis . . . . . . . . . . . . . . . . 80

5. SOFTWARE-DEFINED RADIO EXPERIMENTATIONS IN APCO-25 AND PUBLIC SAFETY LTE FREQUENCY BANDS . . . . . . . . . . . . . 81

5.1 Capturing APCO-25 signals . . . . . . . . . . . . . . . . 81

5.2 Capturing LTE Band Class $14 \ldots \ldots$. . . . . . . . . . . . . 83

6. ANALYSIS OF FEICIC IN TWO-TIER AG-HETNET $\ldots \ldots \ldots$. . . . 86

6.1 System Model . . . . . . . . . . . . . . . . . . . . . 8 86

6.1 .1 Path Loss Model . . . . . . . . . . . . . . . . . . . . . . . 88

6.1.2 3GPP Release 10/11 Inter-Cell Interference Coordination . . . . . . . . 91

6.1 .3 UE Association and Scheduling . . . . . . . . . . . . . . . . . . . 93

6.2 UABS Deployment Optimization . . . . . . . . . . . . . . . 96

6.2.1 Genetic Algorithm based UABS Deployment Optimization . . . . . . . 96 
6.2.2 UABS Deployment in a Hexagonal Grid . . . . . . . . . . . . . . . . 98

6.3 Simulation Results . . . . . . . . . . . . . . . . . . . . . . . 100

6.3.1 5pSE with UABSs Deployed on a Hexagonal Grid . . . . . . . . . . . 100

6.3.2 5pSE with GA Based UABS Deployment Optimization . . . . . . . . 106

6.3.3 Performance Comparison Between Fixed (Hexagonal) and Optimized UABS Deployment with eICIC and FeICIC . . . . . . . . . . 109

6.3.4 Comparison of Computation Times for Different UABS Deployment Algorithms . . . . . . . . . . . . . . . . . 111

7. HEURISTIC APPROACH FOR JOINTLY OPTIMIZING FEICIC AND UAV LOCATIONS IN MULTI-TIER LTE-ADVANCED PUBLIC SAFETY

HETNET . . . . . . . . . . . . . . . . . . . . . . . . 114

7.1 System Model . . . . . . . . . . . . . . . . . . . . . . . . . . . . . . 114

7.1 .1 Path Loss Model . . . . . . . . . . . . . . . . . . . . . . . . . 116

7.1.2 Spectral Efficiency with 3GPP Release-10/11 ICIC . . . . . . . . . . 120

7.2 UABS Locations and ICIC Parameter Optimization in AG-HetNet . . . 123

7.2 .1 Heuristic Algorithms . . . . . . . . . . . . . . . . . . . 126

7.3 Simulation Results . . . . . . . . . . . . . . . . . . . . . . . . . . 129

7.3.1 KPI Optimization using Brute Force Technique . . . . . . . . . . . . 130

7.3.2 KPI Optimization using Genetic Algorithm . . . . . . . . . . . . . 134

7.3 .3 KPI optimization using eHSGA . . . . . . . . . . . . . . . . . 137

7.3.4 Performance Comparison of the Algorithms . . . . . . . . . . . . . . 140

8. CONCLUDING REMARKS AND FUTURE WORKS . . . . . . . . . . 145

VITA . . . . . . . . . . . . . . . . . . . . . . 182 


\section{LIST OF FIGURES}

FIGURE

PAGE

1.1 Example for current public safety network structure. . . . . . . . . . . 2

1.2 The PSC scenario with MBS and UABSs constituting an two-tier AGHetNet infrastructure. The MBS can use inter-cell interference coordination techniques defined in LTE-Advanced. The UABSs can dynamically change their position to maintain good coverage and can utilize range expansion bias to take over MBS UEs. . . . . . . .

1.3 The terrestrial nodes (MBS, PBS, and GUE) and aerial nodes (UABS and AUE) constitute the three-tier AG-HetNet. The MBS and PBS can use inter-cell interference coordination techniques defined in LTEAdvanced. The PBSs and mobile UABSs can utilize range expansion bias to offload UEs in network congested areas. . . . . . . . . . . . 5

3.1 An example for a LMRS network [1] . . . . . . . . . . . . . . . . 20

3.2 APCO-25 portable and mobile radios in the United States. The radios are from (a), (b) Motorola Solutions [2], (c) Kenwood [3], and (d) Harris [4]. The radios (a), (c) and (d) are LMRS capable whereas radio (b) is a hybrid equipment with LMRS and LTE capabilities . . 21

3.3 TETRA portable and mobile radios from (a) Motorola Solutions [5], (b) Sepura $[6]$, and (c) Hytera $[7] \ldots \ldots \ldots \ldots \ldots . \ldots \ldots$

3.4 $700 \mathrm{MHz}$ band plan for public safety services in the United States. A, $\mathrm{B}, \mathrm{C}$, and $\mathrm{D}$ denote different bands in the $700 \mathrm{MHz}$ spectrum. . . . . 30

3.5 Band class 14 plan for public safety services. D block will be reallocated for use by public safety entities as directed by Congressional mandate [8]. $758 \mathrm{MHz}-768 \mathrm{MHz}$ would be the downlink and $788 \mathrm{MHz}-798$ $\mathrm{MHz}$ would be uplink public safety frequency allocation in band class 14. Bands A and B are the guard bands of $1 \mathrm{MHz}$ each. . . . . . . . 30

3.6 Current $800 \mathrm{MHz}$ band plan for public safety services [9], [10] . . . . . . 32

3.7 SMR, industrial, scientific and medical (ISM), paging, and fixed microwave are the radio bands in $900 \mathrm{MHz}$ spectrum plan [10], [11]. . . 33

3.8 FirstNet PSC architecture proposed by $[12] . \ldots \ldots$. . . . . . . 36

3.9 LTE band class 14 architecture for FirstNet [13] . . . . . . . . . . . . 38

3.10 VIDA from Harris Corporation is a converge platform that integrates legacy and broadband PSC into a core network [14]. . . . . . . . . 40 
3.11 Proximity service examples as proposed in 3GPP LTE Release 12[15]. Path (a) shows current conventional LTE communication path, (b) shows direct communication with proximity services and (c) shows locally routed communication with proximity services. . . . . . . . 42

3.12 System level representation of the LMRS and the LTE networks for PSC. 44

3.13 APCO-25 channel configuration. . . . . . . . . . . . . . . 44

3.14 LTE channel configuration. . . . . . . . . . . . . . . . . . . 45

3.15 In a single frequency network, base stations transmit the same signal at the same time and over the same frequency channel to UE. In this example MBSFN area, the group of cells perform synchronized eMBMS transmission. These transmitted signals appear as multipath components to the UE. . . . . . . . . . . . . . . 49

3.16 Small cell example for PSC during an emergency scene. . . . . . . . . . 53

4.1 LTE model for NS-3 simulations, based on the LENA project [16]. All the important details of the LTE PHY and MAC protocols are implemented in the NS-3 simulations. . . . . . . . . . . . . 70

4.2 The CDF plot of simulated LTE NS-3 model; plot illustrates average uplink and downlink throughput (Mbits/s) with 25 and 50 UEs. . . . 70

4.3 Aggregated uplink and downlink throughput observed by 20 UEs in the LTE band class 14. UEs experience better throughput when in close proximity with macro-eNodeB. . . . . . . . . . . . . .

4.4 The CDF plot of DL SINR values measured by the UEs placed at cell center, post cell center, pre cell-edge, and cell-edge regions. . . . . . 73

4.5 Inter-cell interference simulation scenario with cell-edge UEs with interference from neighboring cell. Such a scenario may e.g. correspond to two fire trucks or police cars that utilize LTE small cells, and are parked next to each other during an emergency incident. . . . . . . . 74

4.6 The CDF plot of DL SINR values measured by the UEs placed on the edge of a LTE cell 1 of Fig. 4.5. . . . . . . . . . . . . . . . . . . . . . 74

4.7 APCO-25 suite of standards for public safety communication referenced in NS-3 simulation. . . . . . . . . . . . . . . . . 76

4.8 The maximum observed throughput in case of data communication is approximately $580 \mathrm{Kbits} / \mathrm{s}$ for both portable and mobile public safety devices. . . . . . . . . . . . . . . . 77

4.9 SINR CDF plots for APCO-25 portable subscriber units, with increasing distance between the subscriber units. . . . . . . . . . . . . . 78 
4.10 SINR CDF plots for APCO-25 mobile subscriber units, with increasing distance between the subscriber units. . . . . . . . . . . . . . . 78

5.1 Broward County, FL, APCO-25 spectrum monitored using SDR\# software and captured using a HackRF receiver $[17,18]$. . . . . . . . . . 82

5.2 Setup for analyzing band class 14 spectrum. . . . . . . . . . . . . 83

5.3 Public Safety Band class 14 cell detection using LTE-Tracker open source

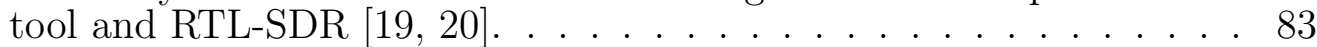

5.4 LTE cell tracker transfer function observed during various RTL-SDR experimentations. The variation in the channel magnitude and phase is observed due to the mobility of user terminal equipped with SDR setup. . . . . . . . . . . . . . . .

6.1 Wireless network coverage before/after a disaster with fixed terrestrial infrastructure. . . . . . . . . . . . . . 8 87

6.2 The CDF of the combined path loss observed from all the base stations in a two-tier AG-HetNet. Dashed lines correspond to the scenario with $50 \%$ of the MBS destroyed, while solid lines correspond to the scenario with $97.5 \%$ of the MBS destroyed.

6.3 LTE-Advanced frame structures for time-domain ICIC in two-tier AGHetNet. Certain UABS subframes are protected from MBS. . . . . . 92

6.4 An example of a chromosome for FeICIC simulation in two-tier AGHetNet, where the UABS locations, ICIC parameter $\tau, \alpha, \rho$, and $\rho^{\prime}$ are optimized. The ICIC parameter $\beta$ is not optimized and is fixed at $50 \%$ duty cycle. . . . . . . . . . . . . . . . . 96

6.5 Two-tier PSC AG-HetNet after a disaster with UABS locations optimized using the GA (UAV height: $100 \mathrm{~m}$ ) . . . . . . . . . . . . . . . 98

6.6 Two-tier PSC AG-HetNet after a disaster with UABS deployed on a fixed hexagonal grid (UAV height: $100 \mathrm{~m}$ ). . . . . . . . . . . . . . . 100

6.7 5pSE versus $\mathrm{CRE}$ for eICIC and FeICIC techniques in two-tier AGHetNet with SPLM (UABSs deployed on a hexagonal grid). . . . . . 102

6.8 Peak observations for the 5pSE with SPLM (UABSs deployed on a hexagonal grid). . . . . . . . . . . . . . . 103

$6.95 \mathrm{pSE}$ versus CRE for eICIC and FeICIC technique in two-tier with $\mathrm{OH}$ PLM (UABSs deployed on a hexagonal grid). . . . . . . . . . . . . . 104

6.10 Peak observations for the 5pSE with OHPLM (UABSs deployed on a hexagonal grid). . . . . . . . . . . . . . . . 105 
6.11 Peak 5pSE versus optimized CRE for eICIC and FeICIC techniques in two-tier AG-HetNet with SPLM, when the UABS locations and ICIC parameters are optimized using the GA. . . . . . . . . . . . . . 106

6.12 SIR observations for eICIC and FeICIC in two-tier HetNet with OHPLM, when UABS locations are optimized using the GA. . . . . . . 107

6.13 Peak 5pSE versus optimized CRE for eICIC and FeICIC techniques with OHPLM in two-tier AG-HetNet, when the UABS locations and ICIC parameters are optimized using the GA. . . . . . . . . . . . . . 108

$6.145 p S E$ comparisons for eICIC and FeICIC with SPLM in two-tier AGHetNet, when the UABS locations are optimized using the GA and when the UABSs are deployed in a fixed hexagonal grid. . . . . . . . 109

$6.155 \mathrm{pSE}$ comparisons for eICIC and FeICIC with OHPLM in the two-tier AG-HetNet, when the UABS locations are optimized using the GA and when the UABSs are deployed in a fixed hexagonal grid. . . . . 110

6.16 GA simulation runtime using FeICIC and eICIC technique with OHPLM and SPLM for the proposed two-tier AG-HetNet model. . . . . . . . 112

6.17 Fixed hexagonal grid simulation runtime using FeICIC and eICIC technique with OHPLM and SPLM for the proposed two-tier AG-HetNet model. . . . . . . . . . . . . . . . . . . . 112

7.1 The CDF of path loss observed for the communication link between UEs (AUE and GUE) and base-stations (MBS, PBS, and UABS) in a three-tier AG-HetNet. . . . . . . . . . . . . . . 119

7.2 The proposed three-tier reduced power USF/CSF LTE subframes of MBS, PBS, and UABS. Certain UABS subframes are protected from both MBS and PBS, while certain PBS subframes are protected from MBS. . . . . . . . . . . . . . . . 120

7.3 Cell selection and UE association in USF/CSF subframes of MBS, PBS, and UABS in three-tier AG-HetNet. . . . . . . . . . . . . . . . 122

7.4 Three dimensional distribution of ground UEs (GUEs), aerial UEs (AUEs), macro base-stations (MBSs), pico base-stations (PBSs), and unmanned aerial base-stations (UABSs) in three-tier AG-HetNet. The densities and deployment heights each of the wireless nodes are specified in Table 7.2. . . . . . . . . . . . . . . . . . . . . 12

7.5 An example of a chromosome for ICIC simulation in three-tier AGHetNet, where the UABS locations and ICIC parameters $\mathbf{S}_{\mathrm{mbs}}^{\mathrm{ICIC}}, \mathbf{S}_{\mathrm{pbs}}^{\mathrm{ICIC}}$, and $\mathbf{S}_{\text {uabs }}^{\mathrm{ICIC}}$ are jointly optimized. . . . . . . . . . . 126

7.6 The effects of combined CRE at PBS and UABS on the two KPIs of the network, with and without ICIC in three-tier AG-HetNet; when the UABS are deployed at height of $25 \mathrm{~m} . \ldots . . . . . . . . .131$ 
7.7 The effects of combined CRE at PBS and UABS on the two KPIs of the network, with and without ICIC in three-tier AG-HetNet; when

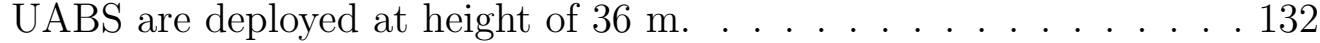

7.8 The effects of combined CRE at PBS and UABS on the two KPIs of the network, with and without ICIC in three-tier AG-HetNet; when UABS are deployed at height of $50 \mathrm{~m} . \ldots 133$

7.9 A combined effect of CRE at PBS and UABS on the two KPIs of the network, with and without ICIC in three-tier AG-HetNet. When UABS are deployed at the height of $25 \mathrm{~m}, 36 \mathrm{~m}$, and $50 \mathrm{~m}$ and while considering GA. . . . . . . . . . . . . . . 136

7.10 A combined effect of CRE at PBS and UABS on the two KPIs of the network, with and without ICIC in three-tier AG-HetNet. When UABS are deployed at the height of $25 \mathrm{~m}, 36 \mathrm{~m}$, and $50 \mathrm{~m}$ and while considering eHSGA. . . . . . . . . . . . . . 139

7.11 Simulation runtime for evaluating a single KPI with/without ICIC techniques in three-tier AG-HetNet; when UABSs are deployed at different heights and using different optimization techniques. . . . . . . . 141 


\section{CHAPTER 1 \\ INTRODUCTION}

\subsection{Public Safety Network}

Public safety organizations protect the well-being of the public in case of natural and man-made disasters, and are tasked with preparing for, planning for, and responding to emergencies. The emergency management agencies include law enforcement agencies, fire departments, rescue squads, emergency medical services (EMS), and other entities that are referred to as emergency first responders (EFR). The ability of EFR to communicate amongst themselves and seamlessly share critical information directly affects their ability to save lives. The communication technologies such as legacy radio system, commercial network $(2 \mathrm{G} / 3 \mathrm{G})$, and broadband (LTE/WiFi) are largely used by the public safety organizations as shown in Fig. 1.1.

Over the recent years, there has been increasing interest in improving the capabilities of public safety communications (PSC) systems. For example, in [21-23], efficient spectrum management techniques, allocation models, and infrastructure options are introduced for PSC scenarios. Reforms on PSC policy have been discussed in [24] and [25], which study the decoupling of spectrum licenses for spectrum access, a new nationwide system built on open standards with consistent architecture, and fund raising approach for the transition to a new nationwide system. As explained in [26], communication of time critical information is an important factor for emergency response. In [27] and [28], insights on cognitive radio technology are presented, which plays a significant role in making the best use of scarce spectrum in public safety scenarios. Integration of other wireless technologies into PSC is studied in [29], with a goal to provide faster and reliable communication capability 


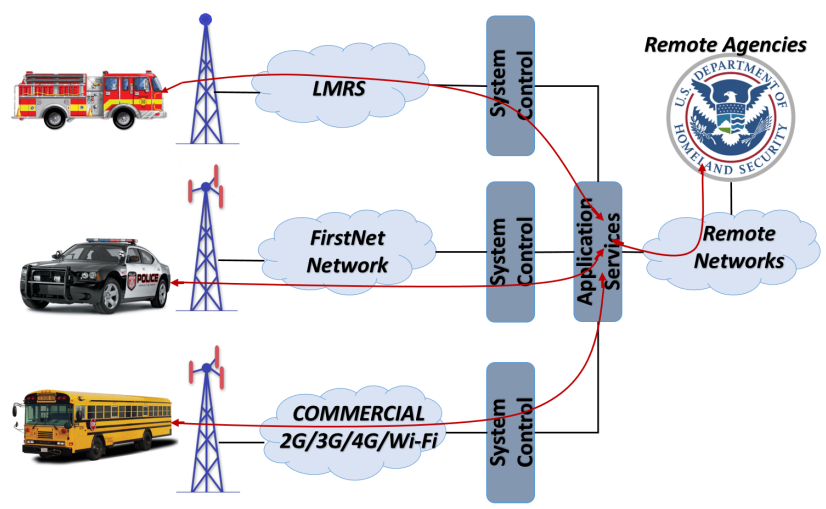

Figure 1.1: Example for current public safety network structure.

in a challenging environment where infrastructure is impacted by the unplanned emergency events.

To further enhance the capabilities of next generation PSC networks, countries such as the United States, the United Kingdom, and Canada are building a 4G Long Term Evolution (LTE) based broadband public safety network [30-32]. In particular, 4G mobile networks have great potential to revolutionize PSC during emergency situations by providing much needed high-speed real-time data, video, and multimedia services along with mission-critical communication.

\subsubsection{Contributions}

In this dissertation, a comprehensive survey and comparative analysis on public safety Land Mobile Radio System (LMRS) and LTE public safety network (PSN) is discussed and covers the following aspects in details.

- Case study of public safety spectrum allocation in the United States, and present an overview of spectrum allocation in VHF, UHF, $700 \mathrm{MHz}, 800$, $\mathrm{MHz}, 900 \mathrm{MHz}$, and $4.9 \mathrm{GHz}$ bands for various public safety entities. 
- Review the LTE-based PSN with the first responders network authority (FirstNet) in the United States as an example, the convergence of LTE-LMR technologies, support for mission-critical PTT (MCPTT) over LTE, and current status of broadband PSNs in different regions of the world.

- Comparative analysis, NS-3 simulation study, and software-defined radio experimentation of LMRS and LTE PSC.

- Comprehensive perspective on how emerging wireless technologies can shape PSN and discuss open research problems for different public safety technologies.

\subsubsection{Publications}

The above works have been published in peer reviewed journal [30] and conference proceeding [33].

\subsection{Role of UAVs in Public Safety HetNets}

Recent developments in reliability and cost-effective hardware have enhanced the drones or unmanned aerial vehicles (UAV) capabilities such as mobility, locationaware connectivity, deployment flexibility in three-dimensional (3D) space, and enabling ubiquitous and non-line-of-sight connectivity. In particular, with minimum interdependencies and at low cost UAVs are deployed as unmanned aerial basestations (UABSs) to meet the mobile data and coverage demands and to restore damaged infrastructure by relieving the pressure on the terrestrial networks and reducing the cost of dense small cell deployments [30, 34-36]. For example, in the aftermath of Hurricane Maria, AT\&T deployed cell on wings (COW) drone to restore 
long term evolution (LTE) cell coverage in Puerto Rico [37-39]. On the other hand, Verizon has been testing a flying cell site that provides LTE coverage of one-mile range [39].

In a broader context, the use of UAV as aerial user equipment (AUEs) has enabled smart city applications such as traffic monitoring, data collection from Internet-of-Things (IoT) nodes, and public safety applications such as search and rescue, and remote location sensing. In the most recent Kilauea volcano eruption, the first responders were able to search and rescue a Hawaiian man using a UAV [40]. Such vast applications have enabled the recent works to study the feasibility of deploying AUEs in collaboration with existing LTE-Advanced infrastructure in [40-49]

\subsubsection{Contributions}

In this dissertation, we integrate the UAVs as both AUEs and UABSs into the existing LTE-Advanced terrestrial network infrastructure and provide system-level understanding to both modify and extend this air-ground HetNet (AG-HetNet). We define and simulate an AG-HetNet system model for an urban environment with public safety LTE band class 14 in the following scenarios:

- In a two-tier AG-HetNet with macro base stations (MBSs), UABS, and ground user equipment (GUEs), we simulate a mock emergency situation by randomly removing MBSs as shown in Fig. 1.2, to study the impact of interference and cell range expansion (CRE) when the UABSs are deployed.

- In a three-tier AG-HetNet with MBS, pico base stations (PBSs) UABS, GUEs, and ground user equipment (GUEs), we study the feasibility of deploying UAVa as both UABS and AUEs with an existing LTE-Advanced terrestrial infrastructure. Furthermore, the investigation of critical aspects such as the 


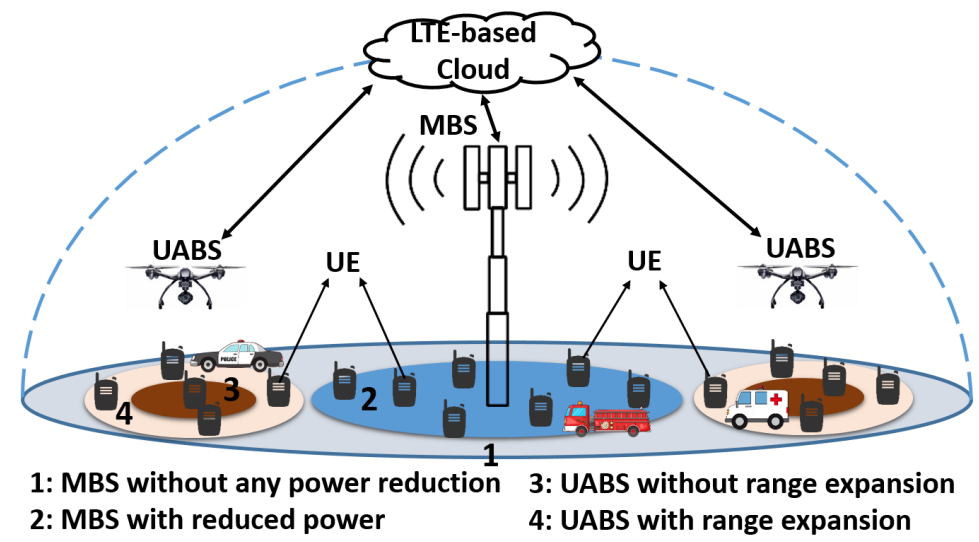

Figure 1.2: The PSC scenario with MBS and UABSs constituting an two-tier AGHetNet infrastructure. The MBS can use inter-cell interference coordination techniques defined in LTE-Advanced. The UABSs can dynamically change their position to maintain good coverage and can utilize range expansion bias to take over MBS UEs.

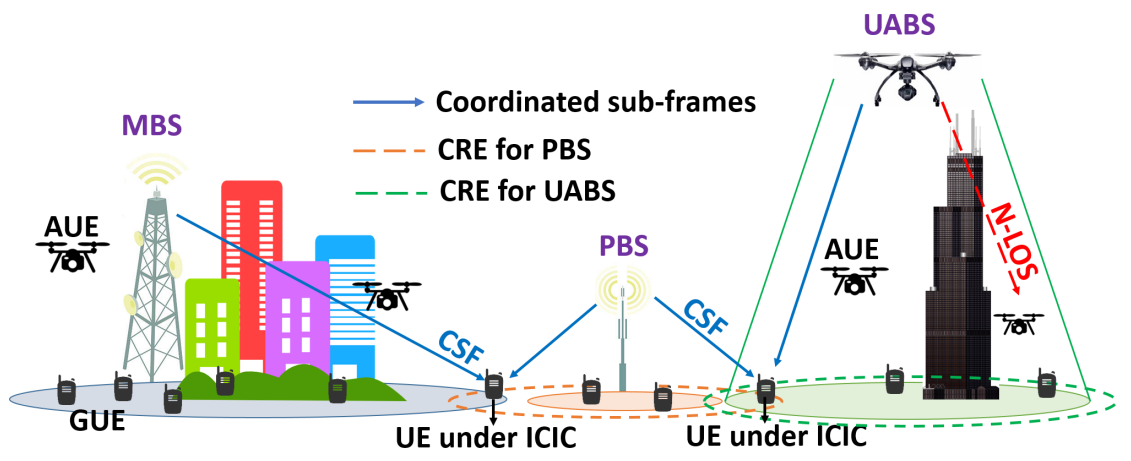

Figure 1.3: The terrestrial nodes (MBS, PBS, and GUE) and aerial nodes (UABS and AUE) constitute the three-tier AG-HetNet. The MBS and PBS can use intercell interference coordination techniques defined in LTE-Advanced. The PBSs and mobile UABSs can utilize range expansion bias to offload UEs in network congested areas.

inter-cell interference, channel modeling support, spectral efficiency, and coverage probability is extended to cover both UABSs and AUEs as part of the AG-HetNet 


\subsubsection{Publications}

The above works have been published in peer reviewed journal $[30,36]$ and conference proceeding $[47,50]$. One of the works has been submitted to peer-review journal and is under review [51]. The author has jointly worked as a co-author in two other relevant studies [35, 42, 49, 52].

\subsection{Capacity and Coverage Enhancement}

Coverage probability and fifth percentile spectral efficiency (5pSE) are the key performance indicators (KPIs) that are considered when designing a AG-HetNet for PSC. For example, FirstNet in the United States is designing a PSC network, which is required to minimize coverage gaps and attain at least $95 \%$ coverage of the geographical area and human population [53] with broadband rates. However, given the limited spectrum allocation for LTE-based PSC, the usage of high-speed real-time data, video, and multimedia services would need improved channel capacity quality, and spectral efficiency (SE) [30, 54].

To this end, the deployment of LTE-Advanced small cells such as the PBS and UABS are increasingly becoming popular to provide improved spectral capacity and extend network coverage $[35,55-58]$. Due to their low transmission power, the small cells are unable to associate a substantial number of UEs compared to that of MBSs. Therefore, we consider the CRE technique defined in 3GPP Release-8 at small cells to extend the network coverage and increase capacity by offloading traffic from congested cells. Although an adverse side effect of CRE includes increased interference at UEs in the cell-edge or CRE region and also limit the overall PSC network SE $[35,58-60]$ 


\subsubsection{Contributions}

In this dissertation, the following innovative techniques for capacity and coverage enhancement of a AG-HetNet will be discussed and analyzed in details

- Inter-cell interference coordination techniques defined in the 3GPP Release10/11 have the ability to improve the channel conditions for the users (AUEs

and GUEs) experiencing server interference form the neighbouring base-stations. The investigation in this dissertation mainly focus on time-domain ICIC techniques in 3GPP. Enhanced ICIC (eICIC) technique defined in 3GPP Release10 uses ABSs which require the MBS to completely blank the transmit power on the physical downlink shared channel (PDSCH) resource elements. This separates the radio frames into coordinated subframes (CSF) and uncoordinated subframes (USF). On the other hand, 3GPP Release-11 defines furtherenhanced ICIC (FeICIC), where the data on PDSCH is still transmitted but at a reduced power level.

- UABSs have the ability to dynamically reposition itself in AG-HetNet environment. By deploying the UABS at optimal location in a AG-HetNet can further enhance the overall capacity and coverage gains. To to end, the contribution of the study is to address this challenge of interference mitigation by using and jointly optimizing the UABS location and AG-HetNet parameters (ICIC and CRE) using the brute-force technique and heuristics approach to achieve maximum capacity and coverage gains.

\subsubsection{Publications}

The above works have been published in peer reviewed journal [36] and conference proceeding $[47,50]$. One of the works has been submitted to peer-review journal 
and is under review [51]. The author has jointly worked as a co-author in two other relevant studies $[35,52]$.

\subsection{Joint Optimization of UABS Locations and ICIC Pa- rameters}

Recent advancements in UAV technology has enabled the possibility of deploying small cells such as UABSs mounted with a communication system. UABSs such as balloons, quadcopters, and gliders equipped with LTE-Advanced capabilities can be utilized to further enhance the capabilities of LTE-based HetNets. The ability of UABSs to dynamically reposition in a HetNet environment can improve the overall SE of the network by filling the coverage gaps and offloading UEs in high-traffic

regions. However, due to their low transmission power, the UABSs are unable to associate a larger number of UEs compared to that of MBSs. By using the cell range expansion (CRE) technique defined in 3GPP Release-8, UABSs can associate a large number of UEs by offloading traffic from the MBSs/PBSs. A negative side effect of CRE includes increased interference in the downlink of cell-edge UEs or the UEs in CRE region of the UABS. Hence, for effective utilization of air-borne small cell, it is of critical nature to optimize the locations of UABSs and ICIC parameters of AG-HetNet to maximize the overall spectral efficiency and coverage probability gains.

\subsubsection{Contributions}

In this dissertation, the following optimization techniques will be considered for joint optimization of UABS locations and ICIC parameters of a AG-HetNet. The 
performance comparison in terms of KPI and computational complexity gains is also discussed in detail.

- Brute-force technique is a lower complexity alternative to optimizing UABS locations. We consider deploying the UABSs on a hexagonal grid, where the positions of UABSs are deterministic and optimize the ICIC parameters of the AG-HetNet using the brute-force.

- Genetic Algorithm (GA) is a suitable meta-heuristic technique that relies on bio-inspired approach that uses mutations, crossovers, and selections of chromosomes, for finding optimum or close to optimum solution of a search problem. However, GA has limitations in terms of low convergence speed and requires high computation time

- Elitist harmony search algorithm based on the genetic algorithm (eHSGA) is a hybrid approach between harmony search and GA to acquire potential enhancements in performance.

\subsubsection{Publications}

The above works have been published in peer reviewed journal [36] and conference proceeding $[47,50]$. One of the works has been submitted to peer-review journal and is under review [51]. The author has jointly worked as a co-author in two other relevant studies $[35,52]$. 


\section{CHAPTER 2}

\section{LITERATURE REVIEW}

\subsection{Public Safety Networks}

There have been relatively limited studies in the literature on PSC that present a comprehensive survey on public safety LMRS and LTE systems. In [61], authors present a discussion on voice over LTE as an important aspect of PSC and then provide a high-level overview of LMRS and LTE technologies for their use in PSC scenario. In $[62,63]$, authors survey the status of various wireless technologies in public safety network (PSN), current regulatory standards, and the research activities that address the challenges in PSC. The ability of LTE to meet the PSN requirements, and classifying possible future developments to LTE that could enhance its capacity to provide the PSC is discussed in [64, 65].

Some of the key references studied in Chapter 3, 4, and 5 related to LMRS, LTE, SDR, and emerging PSC technologies are classified in Table 2.1 and Table 2.2, along with possible research directions.

\subsection{Interference Coordination and Capacity Analysis of two- tier AG-HetNet}

LTE-based HetNets are a well-researched topic, and numerous studies have been carried to enhance the network performance, improve coverage, and mitigate network interference. In [214], a fractional frequency reuse method is used to mitigate interference in a fixed HetNet, improve the indoor coverage, and maximize the network SE by minimizing the UE's outage probability to cell-edge UEs in an OFDMA- 
based LTE HetNet. However, [214] did not consider any of the 3GPP Release-10 and Release-11 ICIC techniques for fixed HetNet deployments.

3GPP Release-10 eICIC and Release-11 FeICIC techniques have been studied in [58, 215, 216] for HetNets. For example, [215] proposes algorithms that jointly optimizes the eICIC parameters, UE cell association rules, and the spectrum resources shared between the macro and fixed small cells. Nevertheless, 3GPP Release-11 FeICIC technique for better radio resource utilization and CRE for offloading a larger number of UEs to small cells was not considered in [215]. The effectiveness of 3GPP Release-10 and Release-11 ICIC techniques with ICIC parameter optimization have been studied in [58], without considering any mobility for small cells.

Recent advancements in UAV technology has enabled the possibility of deploying small cells as UABSs mounted with a communication system. UABSs such as balloons, quadcopters, and gliders equipped with LTE-Advanced capabilities can be utilized to further enhance the capabilities of LTE-based HetNets. The ability of UABSs to dynamically reposition in a HetNet environment can improve the overall SE of the network by filling the coverage gaps and offloading UEs in high-traffic regions. Hence, it is of critical nature to optimize the locations of UABSs in a UAV-based HetNet to optimize SE gains.

Recent studies [217-222] are mainly focused on finding an optimal location of UAVs in the geographical area of interest to meet traffic demands. In [217-220], UAV location optimization have been explored; however, inter-cell interference coordination techniques are not explicitly taken into account. Authors in [35, 150] explore UABS-assisted LTE HetNets, where the UABSs use CRE for offloading users from a macrocell but do not consider any ICIC in the cell expanded region. To maximize the 5pSE of the HetNet, a brute force method is used to find the opti- 
mal UAV locations in [150], while the genetic algorithm is used for optimizing UAV locations in [35].

The effect of interference in a UAV-based network is investigated in [223]. By calculating the optimal distance between the two interfering UAVs, each UAV is positioned at fixed height to maximize the coverage area. However, this UAVbased network is not designed for LTE-Advanced HetNets. A priority-based UE offloading and UE association with mobile small cells for PSC is studied in [224]. To improve the overall system throughput, 3GPP Release-10 eICIC and CRE is taken into account. However, using almost blank subframes (ABS) at an MBS results in under-utilization of radio resources when compared to the use of reduced power FeICIC.

The use of 3GPP Release-10/11 inter-cell interference coordination techniques in a two-tier LTE-Advanced AG-HetNet has not been adequately studied in the literature. The contribution of Chapter 6 is to address this challenge of interference mitigation by using and optimizing FeICIC and CRE techniques and at the same time, optimize the UAV locations using the genetic algorithm to achieve maximum SE.

\subsection{Heuristics Approach used in Joint Optimization of In- terference Coordination and UABS Locations in AG- \\ HetNet}

There have been relatively limited studies in the literature that use heuristic techniques for the joint optimization ICIC parameters and the UABS locations in the AG-HetNet. The feasibility of deploying UAV as both UABS and AUEs with an 
existing LTE-Advanced terrestrial infrastructure and investigating the critical aspects such as the inter-cell interference, channel modeling support, SE, and coverage probability is extended to cover both UABSs and AUEs as part of the AG-HetNet is studied in Chapter 6 and 7. The specific contributions of our work in the context of the existing literature is summarized in Table 2.3.

\subsection{Interference Coordination and Capacity and Coverage Analysis of three-tier AG-HetNet}

The UABS-based communications and networking present research challenges in the field of network planning, optimal 3D deployment, interference management, performance characterization, handover management, and integrating a suitable channel model. However, the existing literature has focused mostly on particular aspects of UABS-based communications and not the air-ground HetNet (AG-HetNet) system as a whole. In particular, [35, 217-220, 220-222, 225-228] have explored UABS location optimization and deployment height, but key aspects such as inter-cell interference coordination (ICIC) techniques and air-ground path loss model are not explicitly taken into account. The effect of interference in a UABS-based network is investigated in [223], by measuring the optimal distance between the two interfering UABSs and positioning each UABS at a fixed height to maximize the coverage area. Whereas, in [214], a fractional frequency reuse method is used to mitigate interference in a fixed HetNet, to improve the indoor coverage and maximize the network SE by minimizing the user equipment (UE) outage probability. Moreover, a priority-based UE offloading and UE association with mobile small cells for public safety communication (PSC) is studied in [224]. However, [214, 223, 224] did 
not consider any of the 3GPP Rel-10 and Rel-11 ICIC techniques for the HetNet deployments.

The effectiveness of 3GPP Rel-10 enhanced ICIC (eICIC) and Rel-11 furtherenhanced ICIC (FeICIC) techniques while taking cell range expansion (CRE) into account has been studied in $[36,52,58,215,216]$ for LTE-Advanced HetNet. Authors in [215] propose algorithms that jointly optimizes the eICIC parameters, UE cell association rules, and spectrum resources shared between the macro base-stations (MBSs) and fixed small cells. However, in [215], the 3GPP Rel-11 FeICIC technique is not considered, which provides better utilization of radio resources and can offload a larger number of UEs to small cells through CRE. The benefits of 3GPP Rel-10 and Rel-11 ICIC techniques with CRE has been investigated in [58], but for a terrestrial LTE-Advanced HetNet. UABS-assisted LTE-Advanced HetNet has been explored in $[36,52]$, where the UABSs uses CRE for offloading users from a macrocell while considering 3GPP Rel-10 and Rel-11 ICIC techniques in the cell expanded region. Furthermore, a brute-force technique and heuristic algorithm is also used to maximize the spectral efficiency gains by optimizing UABS locations and ICIC network parameters. However, the coverage probability of the wireless network is not investigated, and channel modeling designed for aerial vehicles is not taken into account.

When AUEs are deployed as part of existing terrestrial infrastructure, they experience the same interference issues in the downlink and signal degradation due to path loss. And to address these concerns, a relevant investigation is needed, if AUEs has to coexist with LTE-Advanced HetNet effectively. In particular, AUEs applications for smart cities has been studied in [41, 43, 44], and the IoT data are transmitted into LTE base-station or via device-to-device multi-hop communications. However, the coexistence of AUEs with existing terrestrial and aerial nodes is 
not considered. On the other hand, the coverage probability of AG-HetNet serving AUEs is evaluated while considering appropriate aerial propagation model in [234], but does not consider 3GPP Rel-15 enhanced support for aerial vehicles and the impact of interference on the AUEs in AG-HetNet. The effectiveness of 3GPP Rel-15 enhanced support for aerial vehicles, and the interference mitigation to improve the data capacity is investigated in $[45,46]$, while the AUEs coexist in the AG-HetNet. Nonetheless, the study does not investigate 3GPP Rel-10/11 interference mitigation techniques in the downlink of the AUEs and the influence of CRE on AUEs while mitigating interference. 
Table 2.1: Literature overview on existing and emerging PSC technologies and open research directions.

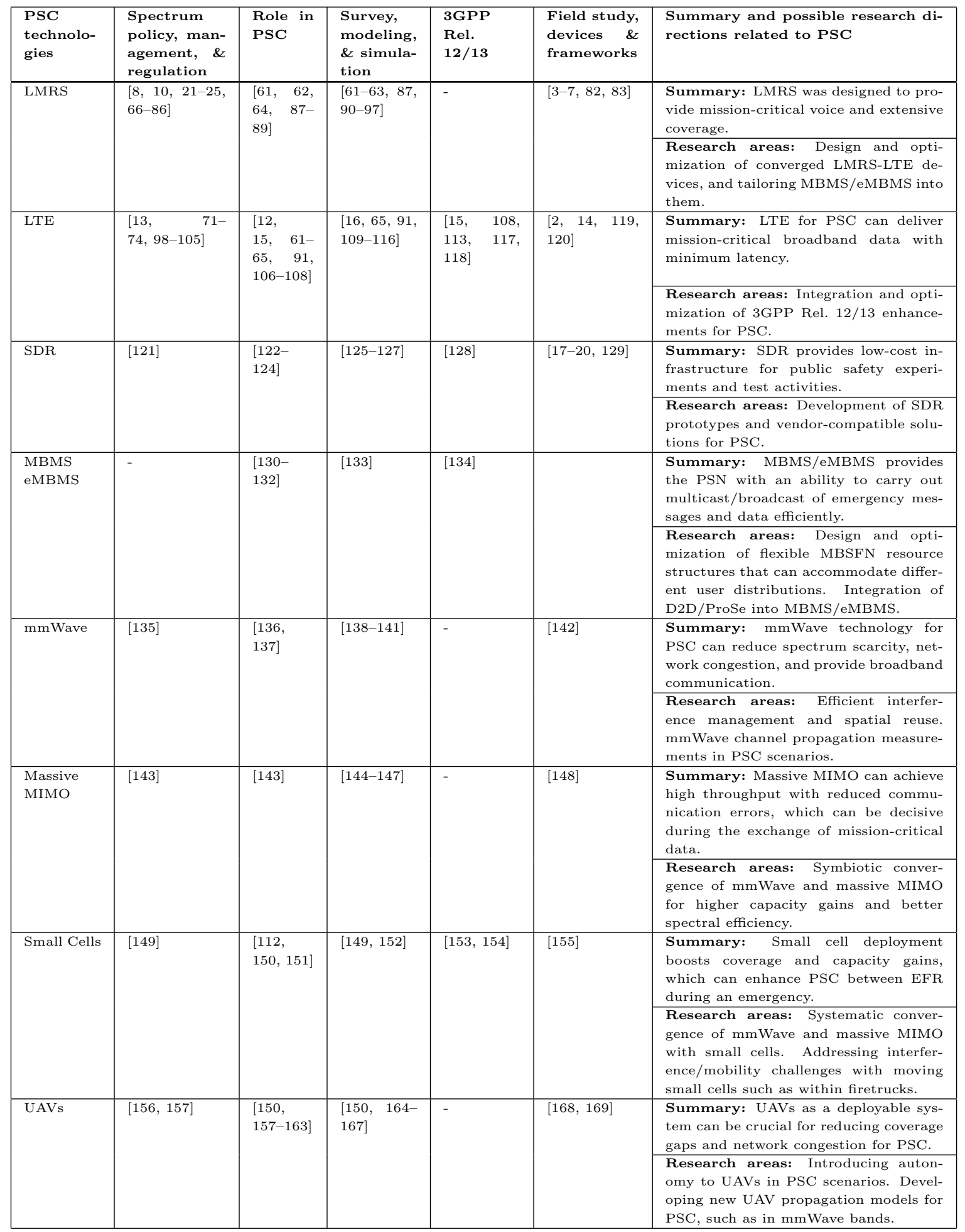


Table 2.2: (Continued) Literature overview on existing and emerging PSC technologies and open research directions.

\begin{tabular}{|c|c|c|c|c|c|c|}
\hline $\begin{array}{l}\text { PSC tech- } \\
\text { nologies }\end{array}$ & $\begin{array}{l}\text { Spectrum } \\
\text { policy, man- } \\
\text { agement, \& } \\
\text { regulation }\end{array}$ & $\begin{array}{l}\text { Role in } \\
\text { PSC }\end{array}$ & $\begin{array}{l}\text { Survey, } \\
\text { modeling, } \\
\& \text { simula- } \\
\text { tion }\end{array}$ & $\begin{array}{l}\text { 3GPP } \\
\text { Rel. } \\
12 / 13\end{array}$ & $\begin{array}{l}\text { Field study, } \\
\text { devices \& } \\
\text { frameworks }\end{array}$ & $\begin{array}{l}\text { Summary and possible research di- } \\
\text { rections related to PSC }\end{array}$ \\
\hline \multirow[t]{2}{*}{$\begin{array}{l}\text { LTE-based } \\
\text { V2X }\end{array}$} & \multirow[t]{2}{*}{-} & \multirow[t]{2}{*}{$\begin{array}{l}{[111,} \\
170-172]\end{array}$} & \multirow[t]{2}{*}[173,174]{} & \multirow[t]{2}{*}[175-177]{} & \multirow[t]{2}{*}{-} & $\begin{array}{l}\text { Summary: LTE-based V2X communi- } \\
\text { cation can assist EFR to be more effi- } \\
\text { cient during disaster management and } \\
\text { rescue operations. }\end{array}$ \\
\hline & & & & & & $\begin{array}{l}\text { Research areas: Evolving eMBMS to } \\
\text { LTE-based V2X needs. Improved pri- } \\
\text { vacy preservation schemes for V } 2 \mathrm{X} \text { par- } \\
\text { ticipants. Interference and mobility } \\
\text { management. }\end{array}$ \\
\hline \multirow[t]{2}{*}{ LAA } & \multirow[t]{2}{*}[178]{} & \multirow[t]{2}{*}[127]{} & \multirow[t]{2}{*}{$\begin{array}{ll}{[151,} & 179- \\
183] & \end{array}$} & \multirow[t]{2}{*}{ [184-189] } & & $\begin{array}{l}\text { Summary: LAA can complement PSC- } \\
\text { that are deployed in licensed bands and } \\
\text { avoid any possibility of network conges- } \\
\text { tion. }\end{array}$ \\
\hline & & & & & & $\begin{array}{l}\text { Research areas: Policies to ensure fair } \\
\text { access to all technologies while coexist- } \\
\text { ing in the unlicensed spectrum. Proto- } \\
\text { cols for carrier aggregation of licensed } \\
\text { and unlicensed bands. }\end{array}$ \\
\hline \multirow[t]{2}{*}{$\begin{array}{l}\text { Cognitive ra- } \\
\text { dio }\end{array}$} & \multirow[t]{2}{*}[68-70,190]{} & \multirow[t]{2}{*}[191]{} & \multirow[t]{2}{*}{ [191-195] } & \multirow[t]{2}{*}{-} & \multirow[t]{2}{*}{$\begin{array}{l}66] \\
66]\end{array}$} & $\begin{array}{l}\text { Summary: Cognitive radio technology } \\
\text { is a viable solution for efficiently using } \\
\text { public safety spectrum. }\end{array}$ \\
\hline & & & & & & $\begin{array}{l}\text { Research areas: Spectral/energy ef- } \\
\text { ficient spectrum sensing and sharing. } \\
\text { Database assisted spectrum sharing. } \\
\text { Prioritized spectrum access. }\end{array}$ \\
\hline \multirow[t]{2}{*}{ WSNs } & \multirow[t]{2}{*}{-} & \multirow[t]{2}{*}{$\begin{array}{l}196, \\
197]\end{array}$} & \multirow[t]{2}{*}{ [198-201] } & \multirow[t]{2}{*}{-} & \multirow[t]{2}{*}{-} & $\begin{array}{l}\text { Summary: Deployment of large-scale } \\
\text { WSN into PSN can increase situational } \\
\text { awareness of EFR and assist in evading } \\
\text { any potential disaster. }\end{array}$ \\
\hline & & & & & & $\begin{array}{l}\text { Research areas: Robust models for } \\
\text { multi-hop synchronization. Tethering } \\
\text { wireless sensor data attached to EFR } \\
\text { equipment. }\end{array}$ \\
\hline \multirow[t]{2}{*}{ IoT } & \multirow[t]{2}{*}{-} & \multirow[t]{2}{*}{$\begin{array}{l}{[202-} \\
204]\end{array}$} & \multirow[t]{2}{*}[205-207]{} & \multirow[t]{2}{*}{-} & \multirow[t]{2}{*}[208]{} & $\begin{array}{l}\text { Summary: Intelligent analysis of real- } \\
\text { time data from IoT devices can enhance } \\
\text { decision-making ability of EFR. }\end{array}$ \\
\hline & & & & & & $\begin{array}{l}\text { Research areas: Tailoring public } \\
\text { safety wearables into IoT, considering } \\
\text { also openness, security, interoperability, } \\
\text { and cost. Formulating policies and reg- } \\
\text { ulations to strike right balance between } \\
\text { privacy and security. }\end{array}$ \\
\hline \multirow[t]{2}{*}{$\begin{array}{l}\text { Cybersecurity } \\
\text { enhance- } \\
\text { ments }\end{array}$} & \multirow[t]{2}{*}{-} & \multirow[t]{2}{*}{$\begin{array}{l}{[209-} \\
212]\end{array}$} & \multirow[t]{2}{*}[213]{} & \multirow[t]{2}{*}{-} & \multirow[t]{2}{*}{-} & $\begin{array}{l}\text { Summary: Securing mission-critical } \\
\text { information has become critical with } \\
\text { real-time data readily flowing through } \\
\text { PSN. Concrete techniques and policies } \\
\text { can help secure mission-critical data } \\
\text { over PSN. }\end{array}$ \\
\hline & & & & & & $\begin{array}{l}\text { Research areas: Securing emergency } \\
\text { medical services and law enforcement } \\
\text { data operating across the LTE-based } \\
\text { PSN. }\end{array}$ \\
\hline
\end{tabular}


Table 2.3: Literature review on heuristics approach used for joint optimization of interference coordination and UABS locations placement in AG-HetNet.

\begin{tabular}{|c|c|c|c|c|}
\hline Reference & $\begin{array}{l}\text { Wireless } \\
\text { nodes }\end{array}$ & $\begin{array}{l}\text { Path loss } \\
\text { model }\end{array}$ & $\begin{array}{l}\text { Optimization } \\
\text { techniques }\end{array}$ & $\begin{array}{l}\text { Optimization } \\
\text { goal }\end{array}$ \\
\hline [35] & MBS, UABS, GUE & Log distance & Brute-force, Genetic algorithm & $\begin{array}{l}\text { UABS locations, spectral efficiency, } \\
\text { coverage }\end{array}$ \\
\hline$[220]$ & MBS, UABS, GUE & Log distance & Neural model & UABS locations \\
\hline$[52]$ & MBS, UABS, GUE & Log distance & $\begin{array}{l}\text { Q-learning, Deep Q-learning, } \\
\text { Brute-force, Sequential algo- } \\
\text { rithm }\end{array}$ & $\begin{array}{l}\text { UABS locations, spectral efficiency, } \\
\text { energy efficiency, interference coor- } \\
\text { dination }\end{array}$ \\
\hline [225] & UABS, GUE & $\begin{array}{l}\text { Log distance, Close- } \\
\text { in mmWave model }\end{array}$ & - & $\begin{array}{l}\text { Spectral efficiency, coverage proba- } \\
\text { bility }\end{array}$ \\
\hline$[226]$ & UABS, GUE & ITU-R P.1410-2 & $\begin{array}{l}\text { Region partition } \\
\text { Backtracking line } \\
\text { algorithm }\end{array}$ & $\begin{array}{l}\text { UABS locations, GUE load balanc- } \\
\text { ing }\end{array}$ \\
\hline$[227]$ & UABS swarm & MIMO channel & $\begin{array}{l}\text { Brute-force, Gradient descent } \\
\text { location optimization }\end{array}$ & UABS locations, spectral efficiency \\
\hline [228] & MBS, GUE, UABS & 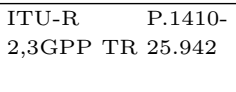 & Deep reinforcement learning & $\begin{array}{l}\text { UABS locations, energy efficiency, } \\
\text { wireless latency, interference coor- } \\
\text { dination }\end{array}$ \\
\hline [229] & UABS, GUE & ITU-R P.1410-2 & Centralized machine learning & UABS locations, energy efficiency \\
\hline [230] & MBS, UABS, GUE & ITU-R P.1410-2 & $\begin{array}{l}\text { Wavelet transform machine } \\
\text { learning }\end{array}$ & $\begin{array}{l}\text { UABS locations, GUE load balanc- } \\
\text { ing }\end{array}$ \\
\hline [231] & MBS, GUE, UABS & ITU-R P.1410-2 & Greedy approach & $\begin{array}{l}\text { UABS 3D-locations, GUE load bal- } \\
\text { ancing }\end{array}$ \\
\hline$[232]$ & UABS, GUE & Free space & $\begin{array}{l}\text { Alternating optimization, Suc- } \\
\text { cessive convex programming }\end{array}$ & $\begin{array}{l}\text { UABS locations, bandwidth alloca- } \\
\text { tion, energy Efficiency }\end{array}$ \\
\hline$[233]$ & UABS, GUE, MBS & MISO channel & $\begin{array}{l}\text { Hybrid fixed-point iteration, } \\
\text { particle swarm optimization }\end{array}$ & $\begin{array}{l}\text { UABS 3D-locations, coverage prob- } \\
\text { ability, interference management, } \\
\text { spectral efficiency }\end{array}$ \\
\hline Chapter 6 & UABS, & $\begin{array}{l}\text { Log distance, } \\
\text { Okumura-Hata }\end{array}$ & $\begin{array}{l}\text { Fixed hexagonal, Brute- } \\
\text { force, Genetic algorithm }\end{array}$ & $\begin{array}{l}\text { UABS locations, spectral effi- } \\
\text { ciency, energy efficiency, inter- } \\
\text { ference coordination }\end{array}$ \\
\hline Chapter 7 & $\begin{array}{l}\text { MBS, } \\
\text { UABS, } \\
\text { AUE }\end{array}$ & $\begin{array}{l}\text { Okumura-Hata, } \\
\text { ITU-R P.1410-2, } \\
\text { 3GPP RP-170779 }\end{array}$ & $\begin{array}{l}\text { Brute-force, Genetic algo- } \\
\text { rithm, eHSGA }\end{array}$ & $\begin{array}{l}\text { UABS locations, spectral ef- } \\
\text { ficiency, coverage probability, } \\
\text { energy efficiency, interference } \\
\text { coordination }\end{array}$ \\
\hline
\end{tabular}




\section{CHAPTER 3}

\section{PUBLIC SAFETY NETWORKS}

Effective emergency and natural disaster management depend on the efficient mission-critical voice and data communication between first responders and victims. LMRS is a legacy narrowband technology used for critical voice communications with limited use for data applications. Recently LTE emerged as a broadband communication technology that has a potential to transform the capabilities of public safety technologies by providing broadband, ubiquitous, and mission-critical voice and data support. For example, in the United States, FirstNet is building a nationwide coast-to-coast public safety network based of LTE broadband technology. This chapter presents a comparative survey of legacy and the LTE-based public safety networks, and discusses the LMRS-LTE convergence as well as mission-critical pushto-talk over LTE. A simulation study of LMRS and LTE band class 14 technologies is provided using the NS-3 open source tool. An experimental study of APCO-25 and LTE band class 14 is also conducted using software-defined radio, to enhance the understanding of the public safety systems. Finally, emerging technologies that may have strong potential for use in public safety networks are reviewed.

\subsection{Introduction}

A PSN is a dedicated wireless network used by emergency services such as police, fire rescue, and emergency medical services (EMS). This network gives better situational awareness, quicker response time to the EFRs, and speed up the disaster response. The scope of a PSN can span over a large geographical area, with vital data flowing into broadband wireless mesh network such as Wi-Fi and LTE. The PSNs are also networked with mobile computing applications to improve the efficiency of the EFR and public well-being. In this chapter, we broadly classify PSNs 


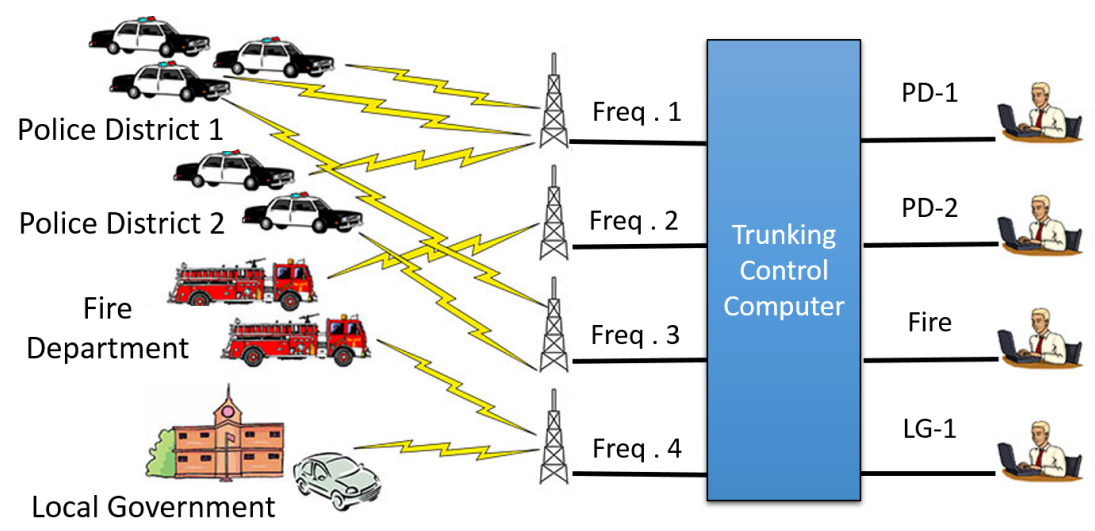

Figure 3.1: An example for a LMRS network [1].

into two categories: LMRS and broadband networks. APCO-25 and TETRA suite of standards falls under LMRS network, while LTE-based broadband PSC network falls under broadband network.

\section{$3.2 \quad$ LMRS Network}

LMRS is a wireless communication system intended for terrestrial users comprised of portables and mobiles, such as two-way digital radios or walkie-talkies. LMRS networks and equipment are being used in military, commercial, and EFR applications as shown in Fig. 3.1. The main goal of LMRS systems are to provide mission critical communications, to enable integrated voice and data communications for emergency response, and to maintain ruggedness, reliability, and interoperability.

APCO-25 and European Terrestrial Trunked Radio (TETRA) are widely used suite of standards for LMRS based digital radio communications. APCO-25 also known as Project 25 (P-25), and it is widely used by federal, state/province, and local public safety agencies in North America. The APCO-25 technology enables public safety agencies enabling them to communicate with other agencies and mutual aid response teams in emergencies. On the other hand, TETRA fulfills the 

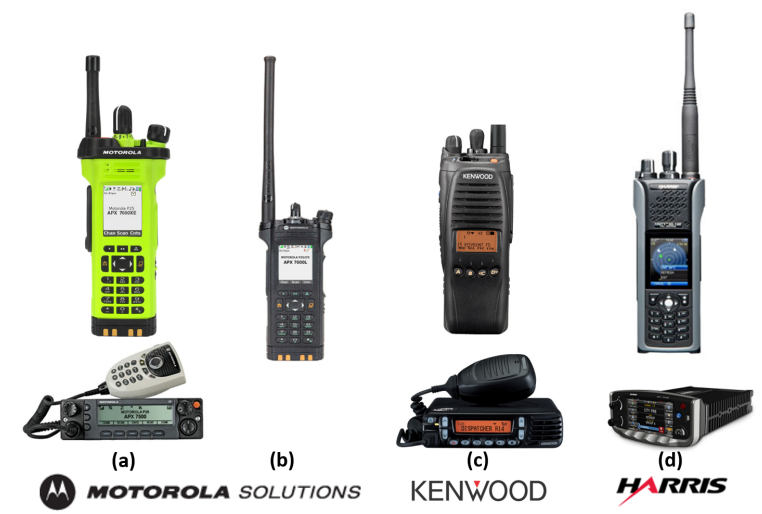

Figure 3.2: APCO-25 portable and mobile radios in the United States. The radios are from (a), (b) Motorola Solutions [2], (c) Kenwood [3], and (d) Harris [4]. The radios (a), (c) and (d) are LMRS capable whereas radio (b) is a hybrid equipment with LMRS and LTE capabilities

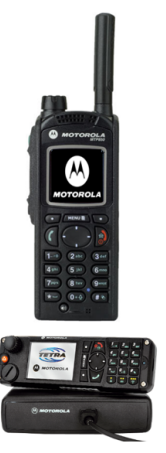

(a)
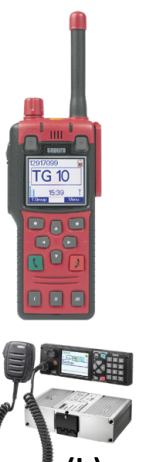

(b)

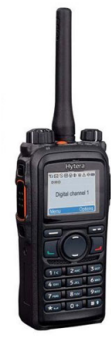

(c)

Figure 3.3: TETRA portable and mobile radios from (a) Motorola Solutions [5], (b) Sepura [6], and (c) Hytera [7].

same role for European and Asian countries. However, these two standards are not interoperable. Fig. 3.2 showcases some of the available APCO-25 portable and mobile digital radios in the market, while Fig. 3.3 showcases TETRA portable and mobile digital radios, procured from the product sheet and websites of the respective companies. 


\subsubsection{APCO-25}

Public safety radios have been upgraded from analog to digital since the 1990s due to the limitations of analog transmission, and implement technological advances by expanding the capabilities of digital radio. Radios can communicate in analog mode with legacy radios, and in either digital or analog mode with other APCO-25 radios. Additionally, the deployment of APCO-25 compliant systems will allow for a high degree of equipment interoperability and compatibility. APCO-25 compliant technology has been deployed in three different phases, where advancements have been gradually introduced [235].

1. In Phase 1, radio systems operate in $12.5 \mathrm{KHz}$ band analog, digital, or mixed mode. Phase 1 radios use continuous 4-level frequency modulation (C4FM) technique, which is a non-linear modulation for digital transmissions. C4FM is a special type of 4FSK modulation as explained in [95] and was developed for the Telecommunications Industry Association (TIA) 102 standard for digital transmission in $12.5 \mathrm{KHz}$. C4FM results in a bit rate of 9600 bits/s. Phase 1 P25-compliant systems are backward compatible and interoperable with legacy systems. P25-compliant systems also provide an open interface to the radio frequency $(\mathrm{RF})$ subsystem to facilitate interlinking between different vendor systems.

2. The goal of Phase $\mathbf{2}$ was to improve the spectrum utilization. Phase 2 introduces a 2 -slot TDMA system which provides two voice traffic channels in a 12.5 KHz band allocation, and doubles the call capacity. It also lays emphasis on interoperability with legacy equipment, interfacing between repeaters and other subsystems, roaming capacity, and spectral efficiency/channel reuse. 
3. Project MESA (Mobility for Emergency and Safety Applications) is a collaboration between the European Telecommunications Standards Institute (ETSI) and the TIA to define a unified set of requirements for APCO - 25 Phase 3. Initial agreement for project MESA was ratified in year 2000. Phase 3 planning activities address the need for high-speed data for public-safety use [235]. Project MESA also aims to facilitate effective, efficient, advanced specifications, and applications that will address public safety broadband communication needs [236].

\subsubsection{TETRA}

TETRA is a digital mobile radio system, which is essentially confined to layers 1-3 of the OSI model. The TETRA system is intended to operate in existing VHF and UHF professional mobile radio frequencies [237], and it has been developed by the European Telecommunications Standards Institute (ETSI). The user needs and technological innovations have led the TETRA standard to evolve with release 1 and release 2 [237].

1. Release $\mathbf{1}$ is the original TETRA standard, which was known as TETRA $\mathrm{V}+\mathrm{D}$ standard. Under this release TETRA radio system supports three modes of operation which are voice plus data $(\mathrm{V}+\mathrm{D})$, direct operation mode $(\mathrm{DMO})$, and packet data optimized (PDO) [238].

The $\mathrm{V}+\mathrm{D}$ is the most commonly used mode, which allows switching between voice and data transmission. Voice and data can be transmitted on the same channel using different slots. The main characteristics of $\mathrm{V}+\mathrm{D}$ are: 1) support for independent multiple concurrent bearer services, 2) support for transmitter preemption, 3) support for several grades of handover, 4) crossed calls are 
minimized by labeling an event on the air interface, 5) support for slot stealing during voice/data transmission, 6) support for different simultaneous access priorities [238].

The DMO, on the other hand, supports direct voice and data transmission between the subscriber units without the base stations, especially when the users are in the outside coverage area. Calls in the DMO can be either clear or encrypted, and full duplex radio communication is not supported under DMO [239].

As a third mode of operation, the PDO standard has been created for occasional data-only, to cater the demands for high volume of data in near future. Location based services and voice are necessary for mission-critical communications and need high volume of data, which can be the beneficiaries of the PDO standard [239].

2. Release $\mathbf{2}$ provided additional functions and improvements to already existing functionality of TETRA. The major enhancement provided by TETRA release 2 is TETRA enhanced data services, which provides more flexibility and greater levels of data capacity [88]. With adaptive selection of modulation schemes, RF channel bandwidths, and coding user bit rates can vary between $10 \mathrm{Kbits} / \mathrm{s}$ to $500 \mathrm{Kbits} / \mathrm{s}$.

Data rate plays a important role in relaying mission-critical information during the emergency situation in timely manner. For example, an emergency scenario monitoring a remote victim would require data rate to support real-time duplex voice/video communication and telemetry. In such a mission-critical scenario, TETRA enhanced data service would play an important role by supporting the applications that need high data rate such as mulitmedia and 
Table 3.1: Emerging wireless broadband communication technologies for creating a PSN[106]. QoS stands for Quality of service, whereas CQI for channel quality indicator.

\begin{tabular}{|l|l|l|l|}
\hline Feature & Wi-Fi & UMTS & LTE \\
\hline Channel width & $20 \mathrm{MHz}$ & $5 \mathrm{MHz}$ & $\begin{array}{l}1.4,3,5,10,15 \\
\text { and 20 MHz }\end{array}$ \\
\hline Frequency bands & $\begin{array}{l}2.4-2.483 \mathrm{GHz}, \\
5.15-5.25 \mathrm{GHz}, \\
5.25-5.35 \mathrm{GHz}\end{array}$ & $700-2600 \mathrm{MHz}$ & $700-2690 \mathrm{MHz}$ \\
\hline Max. data rate & $54 \mathrm{Mbits} / \mathrm{s}$ & $42 \mathrm{Mbits} / \mathrm{s}$ & Up to 300 Mbits/s \\
\hline Range & Up to $100 \mathrm{~m}$ & Up to $10 \mathrm{~km}$ & Up to 30 km \\
\hline Data capacity & Medium & Medium & High \\
\hline Coverage & Intermittent & Ubiquitous & Ubiquitous \\
\hline Mobility support & Low & High & Up to 350 km/h \\
\hline QoS support & $\begin{array}{l}\text { Enhanced dis- } \\
\text { tributed channel } \\
\text { access }\end{array}$ & $\begin{array}{l}\text { QoS classes and } \\
\text { bearer selection }\end{array}$ & $\begin{array}{l}\text { QCI and bearer } \\
\text { selection }\end{array}$ \\
\hline
\end{tabular}

location services. Other TETRA improvements also include adaptive multi-

ple rate voice codec, mixed excitation linear predictive enhanced voice codec, and trunked mode operation range extension, which extended the range for air-ground-air services to 83 kilometers when compared to 58 kilometers in TETRA release 1 [89].

\subsection{LTE Broadband Network}

LTE is a broadband technology that will allow high data rate applications that are not possible to support with LMRS. LTE will enable unprecedented broadband service to public safety agencies and will bring the benefits of lower costs, consumer-driven economies of scale, and rapid evolution of advanced communication capabilities [13].

The Table 3.1, showcases various potential wireless communication standards, that can be used for public safety broadband networks. LTE standard, is developed 
by the $3 \mathrm{GPP}$ as a $4 \mathrm{G}$ broadband mobile communication technology. The main goal of LTE is to increase the capacity and high-speed data over wireless data networks. The technological advances has brought LTE to a performance level close to Shannon's capacity bound [62]. Due to limited code block length in LTE, full SNR efficiency is not feasible. More specifically, LTE performance is less than $1.6 \mathrm{~dB}-2$ $\mathrm{dB}$ off from the Shannon capacity bound as discussed in [240]. With steady increase of investment in broadband services, the LTE technology is soon expected to become most widely deployed broadband communication technology ever. An LTE-based broadband PSN, dedicated solely for the use of PSC can deliver the much needed advanced communication and data capabilities. As an example for LTE-based PSN, we briefly study FirstNet deployment in the United States in Section 3.6.

\subsection{Major Challenges in PSNs}

Even with several technical advancements in PSN, there are major challenges that can hinder efficient operation of EFR. For instance, during a large-scale disaster, different public safety organizations are bound to use different communications technologies and infrastructure at the same time. This can cause major challenges such as, network congestion, low data rates, interoperability problems, spectrum scarcity, and security problems.

\subsubsection{Network Congestion}

In the case of an emergency, network activity spikes up, causing traffic congestion in public safety and commercial networks. Many EFR agencies using IP-based the commercial data services in case of emergency would result in a competition of air time with the general public [87]. The probability of any natural or man- 
made emergency can be assumed as a discrete random event. In case of such events, providing reliable communication becomes critical. Therefore, ensuring that network congestion will not happen in public safety networks during emergency situations is becoming increasingly more important. Emerging technologies such as licensed assisted access (LAA) of LTE to the unlicensed bands, small cells, and UAVs can be used as supplementary solutions during emergency situations to reduce the impact of network congestion [179-181]. Furthermore, addressing the open challenges in the field of mmWave, massive MIMO, and vehicular network system can further help to lower the network congestion. Nevertheless, effective mitigation of network congestion problems during emergency situations remains an open issue.

\subsubsection{Maintaining Ubiquitous Throughput and Connectiv- ity}

The data rate plays a critical role in relaying the information (i.e., voice and data) during the emergency situation in a timely manner. For example, an emergency scenario monitoring a remote victim would require data rate to support real-time duplex voice/video communication, telemetry, and so forth. Another example needing a real-time situational awareness is during fires for firefighters, through the use of streaming video and mission critical voice/data. These examples of mission-critical scenarios as per [24] would need higher data rates and require broadband allocation

of spectrum. Lower data rate limits the usage of data applications, such as multimedia services which have a great potential to improve the efficiency of disaster recovery operations $[92,241]$. The traditional public safety systems was designed to provide better coverage, mission-critical voice, but not peak data capacity. With the advent of $3 \mathrm{GPP}$ specifications, the data rates have been steadily increasing 
and will help in enhancing the capabilities of PSC systems. These data rates can be further increased by making technologies such as mmWave and massive MIMO commercially available. However, 3GPP systems deliver less coverage area when compared to LMRS. Furthermore, they can also experience relatively low data rates and dropped communications at the cell-edge. The coverage and fringe condition in LTE-based PSN can be addressed by the deployment of UAVs or vehicular network system to set up a small cell or virtual cell site. Regardless of the advancements in PSC, maintaining ubiquitously high throughput and connectivity during the emergency situation is still a challenge.

\subsubsection{Interoperability}

Traditionally, interoperability of a radio network means coordinating operating parameters and pre-defined procedures between the intended operators in the network. As discussed in [85], the interoperability failure that occurred during the September 11, 2001 incident was due to the presence of various independent public safety agencies, which resulted in entanglement of systems that were not interoperable. This led to communication failures between various EFRs, and posed a risk to public lives. A possible solution for avoiding any potential interoperability issue includes EFR carrying multiple devices to be effective, which is an expensive strategy and an inefficient use of spectrum resources. Alternatively, communication infrastructure and policies can be evolved for EFR which is a more efficient approach. As per [86], FCC is taking steps in resolving the lack of interoperability in $700 \mathrm{MHz}$ band in a cost-effective manner by leveraging on commercial technologies and infrastructure. 


\subsection{Case Study: Public Safety Spectrum Allocation in the United States}

Public safety operations can be classified mainly based on the applications and usage scenarios, such as mission-critical, military, transportation, utilities, and corporations with large geographical footprint. In this section, we provide a comprehensive overview of VHF, UHF, $700 \mathrm{MHz}, 800 \mathrm{MHz}, 900 \mathrm{MHz}$, and $4.9 \mathrm{GHz}$ public safety band plans and spectrum allocation in the United States. The Federal Communications Commission (FCC) in the United States is a regulatory body for regulating communication by radios, television, wire, satellite, and cable. The FCC has taken steps to ensure that 911 and other critical communication remain operational when a disaster strikes. A major goal of FCC is to enable an operable and interoperable public safety communications (PSC) system in narrowband and broadband spectrum [8].

\subsubsection{MHz Public Safety Spectrum}

The $700 \mathrm{MHz}$ spectrum auction by FCC is officially know as Auction 73 [71]. The signals in $700 \mathrm{MHz}$ spectrum travel longer distances than other typical cellular bands, and penetrate well. Therefore the $700 \mathrm{MHz}$ band is an appealing spectrum to build systems for both commercial and PSN networks. Fig. 3.4a, Fig. 3.4b, Fig. 3.5, and along with Table 3.2 depict the spectrum allocated in the $700 \mathrm{MHz}$ band in the United States. The lower $700 \mathrm{MHz}$ range covers channels (CH) 5259 and 698-746 $\mathrm{MHz}$ frequency, while the upper $700 \mathrm{MHz}$ range covers $\mathrm{CH}$ 60-69 and 746-806 MHz frequency. The 3GPP standards have created four different band classes within $700 \mathrm{MHz}$ band, i.e., band class 12, 13, 14, and 17 . 


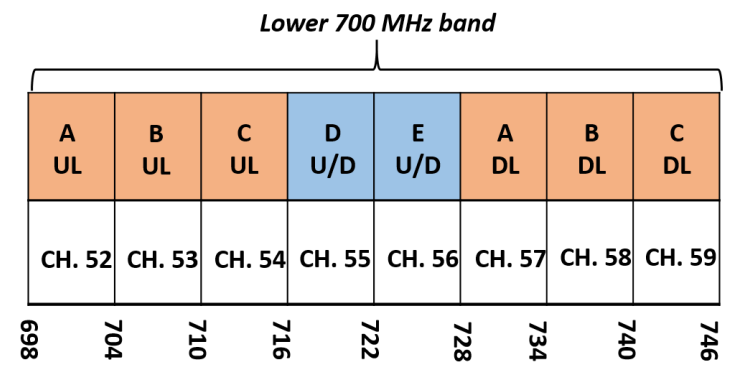

(a) $700 \mathrm{MHz}$ lower band plan. Bands $\mathrm{A}$, $\mathrm{B}$, and $\mathrm{C}$ represent narrowband public safety bands, which are used for voice communications.

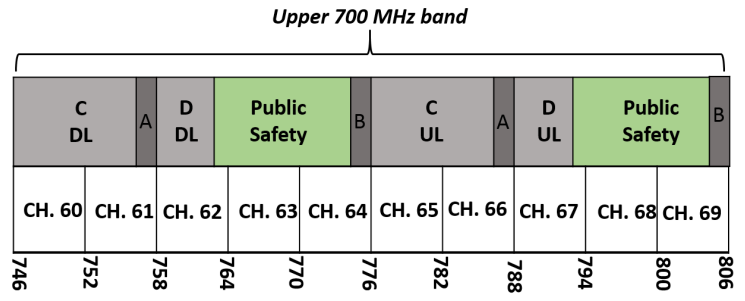

(b) $700 \mathrm{MHz}$ upper band plan. Broadband public safety spectrum is explicitly illustrated, which dedicated for LTE communications.

Figure 3.4: $700 \mathrm{MHz}$ band plan for public safety services in the United States. A, $\mathrm{B}, \mathrm{C}$, and $\mathrm{D}$ denote different bands in the $700 \mathrm{MHz}$ spectrum.

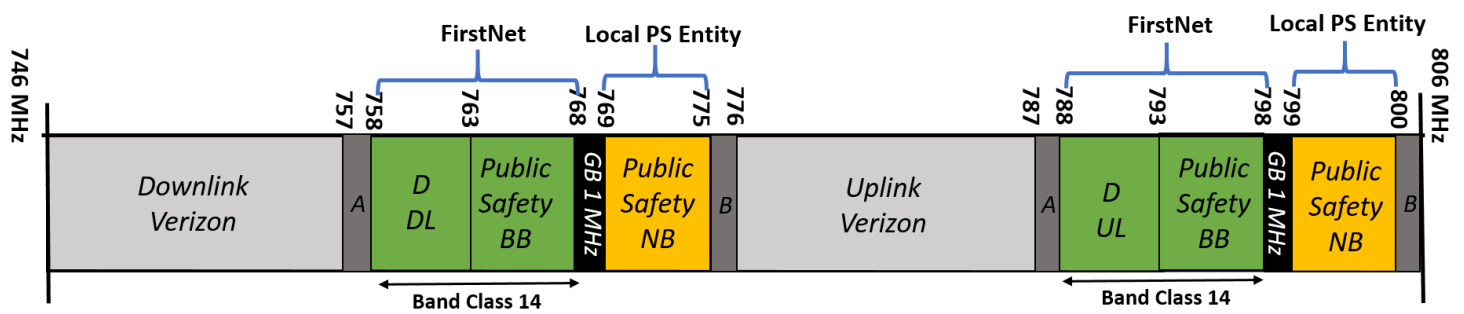

Figure 3.5: Band class 14 plan for public safety services. D block will be reallocated for use by public safety entities as directed by Congressional mandate [8]. $758 \mathrm{MHz}$ - $768 \mathrm{MHz}$ would be the downlink and $788 \mathrm{MHz}$ - $798 \mathrm{MHz}$ would be uplink public safety frequency allocation in band class 14. Bands A and B are the guard bands of $1 \mathrm{MHz}$ each.

Under the current framework, $20 \mathrm{MHz}$ of dedicated spectrum is allocated to PSN in the $700 \mathrm{MHz}$ band as shown in Fig. 2. Green color blocks represent public safety broadband ranging from $758 \mathrm{MHz}$ to $768 \mathrm{MHz}$ with $763 \mathrm{MHz}$ as the center 
Table 3.2: Spectrum allocation of $700 \mathrm{MHz}$ : band class 12, 13, 14, and 17 [242].

\begin{tabular}{|c|c|c|c|c|}
\hline $\begin{array}{c}\text { Band } \\
\text { class }\end{array}$ & $\begin{array}{c}\text { Spectrum } \\
\text { block }\end{array}$ & $\begin{array}{c}\text { Uplink } \\
(\mathbf{M H z})\end{array}$ & $\begin{array}{c}\text { Downlink } \\
(\mathbf{M H z})\end{array}$ & $\begin{array}{c}\text { Band gap } \\
(\mathbf{M H z})\end{array}$ \\
\hline 12 & $\begin{array}{l}\text { Lower block A } \\
\text { Lower block B } \\
\text { Lower block C }\end{array}$ & $698-716$ & $728-746$ & 12 \\
\hline 13 & Upper C block & $777-787$ & $746-756$ & 41 \\
\hline 14 & $\begin{array}{c}\text { Upper D Block and } \\
\text { Public safety allocation }\end{array}$ & $788-798$ & $758-768$ & 40 \\
\hline 17 & $\begin{array}{l}\text { Lower B block } \\
\text { Lower C block }\end{array}$ & $704-716$ & $734-746$ & 18 \\
\hline
\end{tabular}

frequency and $788 \mathrm{MHz}$ to $798 \mathrm{MHz}$ with $793 \mathrm{MHz}$ as the center frequency. Narrowband spectrum is represented in orange color blocks ranging from $769 \mathrm{MHz}$ to $775 \mathrm{MHz}$ and $799 \mathrm{MHz}$ to $805 \mathrm{MHz}$. This part of $700 \mathrm{MHz}$ public safety band is available for local public safety entity for voice communication. Guard bands (GB) of $1 \mathrm{MHz}$ are placed between broadband, narrowband and commercial carrier spectrum to prevent any interference. A part of the $700 \mathrm{MHz}$ public safety narrowband spectrum is also available for nationwide interoperable communications. In particular, interoperability is required to enable different governmental agencies to communicate across jurisdictions and with each other, and it is administered at state level by an executive agency [243].

The deployment strategy of EFR varies depending on the magnitude of the emergency. As the emergency grows, the demand for additional tactical channels would grow too. These tactical channels would render necessary short-range communications at the emergency scene, without taxing the main dispatch channels. This implies the need for additional bandwidth allocation to EFR. However, before reallocation of D block, there was none to little bandwidth available for PSC in 700 $\mathrm{MHz}$ spectrum. The only option for the EFR would be to use commercial network for PSC. However, commercial networks are not fully capable of mission-critical 


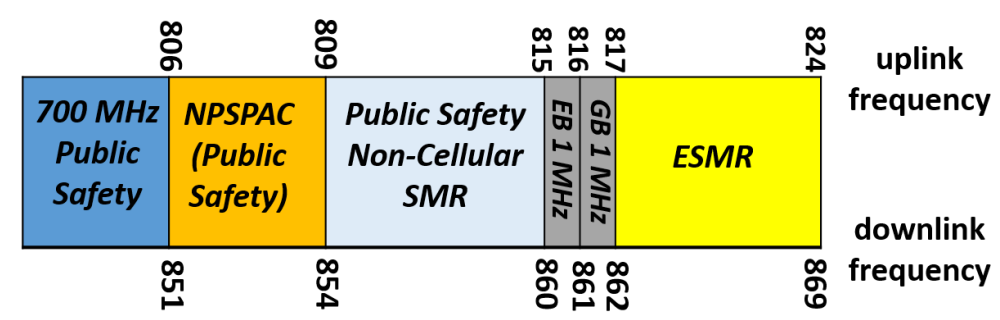

Figure 3.6: Current $800 \mathrm{MHz}$ band plan for public safety services [9], [10].

communication. Therefore, the full allocation of D Block i.e., $20 \mathrm{MHz}$ of $700 \mathrm{MHz}$ was critical to public safety [72]. With reallocation of the D Block to public safety, LTE-based PSN in the $700 \mathrm{MHz}$ would be beneficial to both commercial and PSN. Conversely, the interoperability and roaming between a commercial and PSN during the emergencies without seriously compromising quality of service for commercial users is an ongoing discussion [73].

\subsection{2 $800 \mathrm{MHz}$ Public Safety Spectrum}

The $800 \mathrm{MHz}$ public safety band is currently configured as illustrated in Fig. 3.6. It houses public safety spectrum allocation, commercial wireless carriers, and private radio systems. National Public Safety Planning Advisory Committee (NPSPAC) has come up with guidelines for $800 \mathrm{MHz}$ band and has uplink channels allocated in $806 \mathrm{MHz}-809 \mathrm{MHz}$ and downlink in $851 \mathrm{MHz}-854 \mathrm{MHz}$. Various regional public safety planning committees administer $800 \mathrm{MHz}$ NPSPAC spectrum. Specialized mobile radio (SMR) and enhanced specialized mobile radio (ESMR) can be either analog or digital trunked two-way radio system. SMR channels are allocated in $809 \mathrm{MHz}-815 \mathrm{MHz}$ and downlink in $854 \mathrm{MHz}$ - $860 \mathrm{MHz}$. Expansion band (EB) and GB are of $1 \mathrm{MHz}$ each, which are allocated between $815 \mathrm{MHz}-817 \mathrm{MHz}$. The 


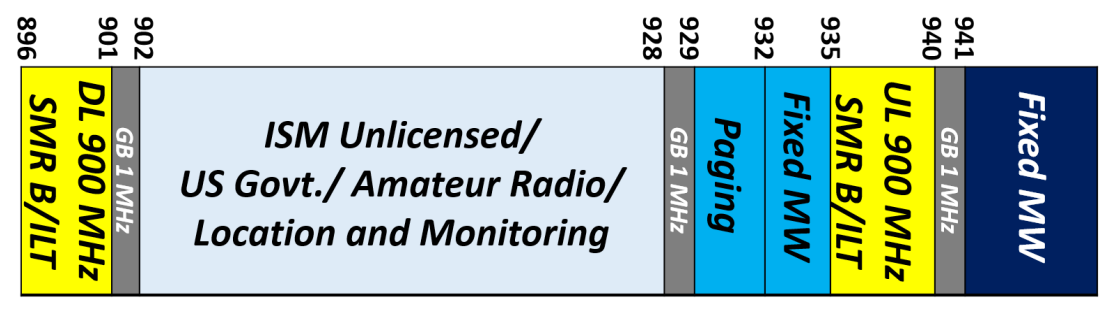

Figure 3.7: SMR, industrial, scientific and medical (ISM), paging, and fixed microwave are the radio bands in $900 \mathrm{MHz}$ spectrum plan [10], [11].

ESMR channels are allocated in the $817 \mathrm{MHz}-824 \mathrm{MHz}$ uplink and $862 \mathrm{MHz}-869$ $\mathrm{MHz}$ for the downlink [9], [10].

\subsubsection{MHz Public Safety Spectrum}

The FCC allows utilities and other commercial entities to file licenses in $900 \mathrm{MHz}$ business and industrial land transport (B/ILT) and the $900 \mathrm{MHz}$ spectrum band plan is shown in Fig. 3.7. The new licenses in the $900 \mathrm{MHz}$ B/ILT band are now allowed on a site-by-site basis for base mobile operator for various commercial (manufacturing, utility, and transportation) and non-commercial (medical, and educational) activities [8], [76].

\subsubsection{VHF and UHF Public Safety Spectrum}

All public safety and business industrial LMRS are narrowbands and operate using $12.5 \mathrm{KHz}$ technology [8]. The operating frequencies for VHF low band ranges between $25 \mathrm{MHz}$ - $50 \mathrm{MHz}$, VHF high band between $150 \mathrm{MHz}$ - $174 \mathrm{MHz}$, and UHF band between $421 \mathrm{MHz}-512 \mathrm{MHz}$ [8]. Licensees in the private land mobile VHF and UHF bands have previously operated on channel bandwidth of $25 \mathrm{KHz}$. As of January 1, 2013 FCC has mandated that all existing licenses in VHF and UHF bands must operate on $12.5 \mathrm{KHz}$ channel bandwidth. Narrowbanding of VHF 
and UHF bands has ensured channel availability, and creation of additional channel capacity within the same radio spectrum. Narrowbanding will result in efficient use of spectrum, better spectrum access for public safety and non-public safety users, and support more users [76-78].

Previously, the frequencies from $470 \mathrm{MHz}$ - $512 \mathrm{MHz}$ were designated as UHF-TV sharing frequencies and were available in certain limited areas of the United States. Public safety in urbanized area such as Boston (MA), Chicago (IL), Cleveland (OH), Dallas/Fort Worth (TX), Detroit (MI), Houston (TX), Los Angeles (CA), Miami (FL), New York (NY), Philadelphia (PA), San Francisco/Oakland (CA), and Washington DC were allowed to share UHF-TV frequencies and were governed by FCC rules 90.301 through 90.317. However, as of February 22, 2012 legislation was enacted to reallocate spectrum in the $\mathrm{D}$ Block within the $700 \mathrm{MHz}$ band for public safety broadband operation. The legislative act prompted FCC to impose a freeze on new T-Band licenses or any modifications to existing licenses. Furthermore, public safety operation is needed to vacate the T-Band spectrum by the year 2023 [81].

\subsection{GHz Public Safety Spectrum}

In year 2002, FCC allocated $50 \mathrm{MHz}$ of spectrum in the $4940 \mathrm{MHz}-4990 \mathrm{MHz}$ band (i.e., $4.9 \mathrm{GHz}$ band) for fixed and mobile services. This band supports a wide variety of broadband applications such as Wireless LAN for incident scene management, mesh network, WiFi hotspots, VoIP, temporary fixed communications, and permanent fixed point-to-point video surveillance [76, 82-84].

Table 3.3, illustrates the public safety spectrum bands, frequencies and the respective users as an example in the state of Florida. 
Table 3.3: Public safety spectrum bands, frequency, and the respective users in the state of Florida. Primary control channels are denoted by (c) and alternate control channels by (a) [79-81].

\begin{tabular}{|c|c|c|}
\hline Spectrum & Frequency $(\mathrm{MHz})$ & Description \\
\hline \multirow[t]{2}{*}{ VHF } & 155.70000 & $\begin{array}{l}\text { Law Dispatch, City of Homestead, FL. } \\
\text { Police main channel } 1 .\end{array}$ \\
\hline & $\begin{array}{l}151.07000,154.81000, \\
155.01000,155.65500, \\
\text { and } 156.21000\end{array}$ & $\begin{array}{l}\text { Law Tactical, City of Homestead, FL. } \\
\text { Police channel } 2-6 .\end{array}$ \\
\hline \multirow[t]{2}{*}{ UHF } & & $\begin{array}{l}\text { Fire-Talk, and local government entity. Miami-Dade county, } \\
\text { FL. }\end{array}$ \\
\hline & $\begin{array}{l}453.13750 \\
453.45000\end{array}$ & $\begin{array}{l}\text { Digital-A channel. } \\
\text { Digital-B channel. }\end{array}$ \\
\hline UHF-TV & $470-476$ & $\begin{array}{l}\text { Miami public safety operations. TV channel } 14 \text { is designated } \\
\text { for LMRS use in Miami urban area. }\end{array}$ \\
\hline \multirow[t]{2}{*}{$700 \mathrm{MHz}$} & & $\begin{array}{l}\text { FL Statewide, Statewide Law Enforcement Project } 25 \text { Radio } \\
\text { System (SLERS P25). System type APCO-25 Phase I, sup- } \\
\text { porting voice over common air interface. }\end{array}$ \\
\hline & $\begin{array}{l}773.59375(\mathrm{c}), 773.84375(\mathrm{a}) \\
\text { and } 774.09375(\mathrm{a})\end{array}$ & Orange county, Fort White, FL. \\
\hline $800 \mathrm{MHz}$ & $\begin{array}{l}851.05000, \quad 853.87500, \\
855.11250, \quad 856.16250, \quad \text { and } \\
858.81250(\mathrm{c})\end{array}$ & $\begin{array}{l}\text { Broward county, Coral Springs FL has Coral Springs public } \\
\text { safety (Project-25). System type APCO- } 25 \text { Phase I, support- } \\
\text { ing voice over common air interface. }\end{array}$ \\
\hline \multirow[t]{2}{*}{$900 \mathrm{MHz}$} & $902-928$ & $\begin{array}{l}\text { Radio spectrum is allocated to amateur radio i.e., amateur } 33 \\
\text { centimeters band. It is also used by ISM and low power unli- } \\
\text { censed devices. Example Motorola DTR650 FHSS (frequency- } \\
\text { hopping spread spectrum) digital two-way radio. }\end{array}$ \\
\hline & $932-935$ & Government and private shared operation, fixed system. \\
\hline $4.9 \mathrm{GHz}$ & $4940-4990$ & $\begin{array}{l}\text { Mission Critical Solutions, has designed a dual radio Wi-Fi } \\
\text { mesh network for the city of Hollywood, FL called as "Wire- } \\
\text { less Hollywood". Each of the strategically located internet } \\
\text { access points is a two-radio system that uses a } 4.9 \mathrm{GHz} \text { for } \\
\text { law enforcement and emergency first responders. The mesh } \\
\text { network showed a } 96.3 \text { percent street level coverage on the } \\
4.9 \mathrm{GHz} \text { safety band, and each access point had a RSSI value } \\
\text { above }-79 \mathrm{dBm}[83] \text {. }\end{array}$ \\
\hline
\end{tabular}

\subsection{Case Study: LTE-based FirstNet PSN}

In January 26, 2011, the FCC in the United States adopted a Third Report and Order and Fourth Further Notice of Proposed Rulemaking (FNPRM). In the third report and order, the FCC mandates the $700 \mathrm{MHz}$ public safety broadband network operators to adopt LTE (based of 3GPP Release 8 specifications) as the broadband technology for nationwide public safety broadband network (NPSBN). The Fourth 


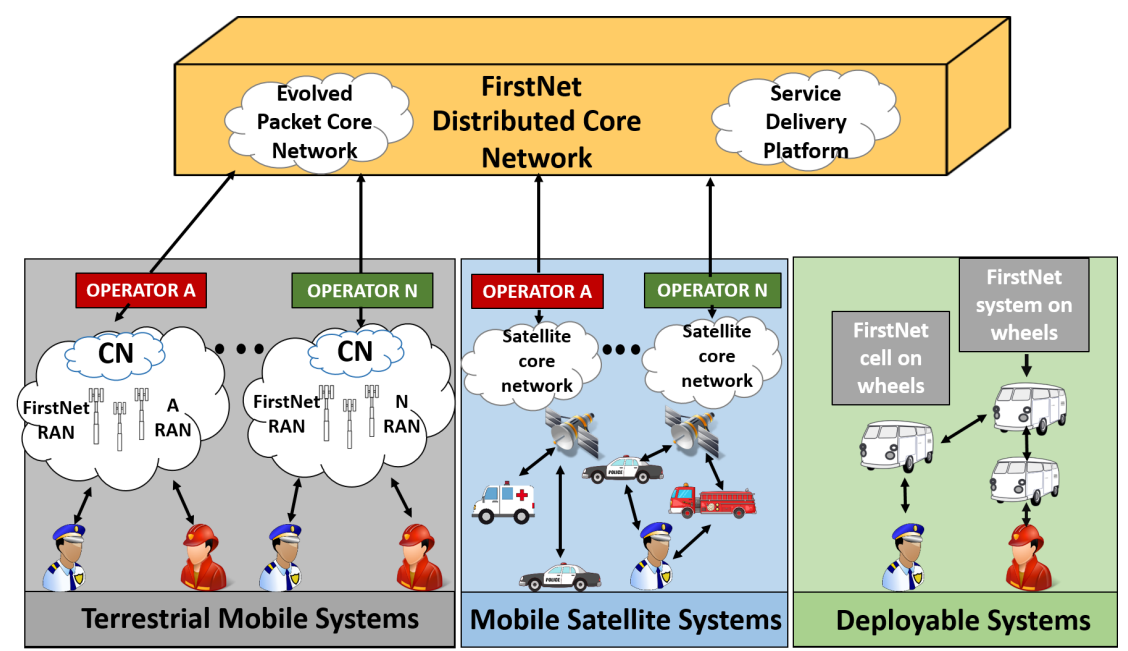

Figure 3.8: FirstNet PSC architecture proposed by [12].

Table 3.4: A variety of deployable technologies for FirstNet [172].

\begin{tabular}{|c|c|c|c|c|c|}
\hline Characteristics & VNS & COLTS & COW & SOW & DACA \\
\hline Capacity & Low/medium & Medium & High & High & Low/medium \\
\hline Coverage & $\begin{array}{lr}\text { Small } & \text { cells } \\
\text { such } & \text { as } \\
\text { pico } & \text { and } \\
\text { femto } & \end{array}$ & $\begin{array}{l}\text { Cell size can be } \\
\text { either macro, or } \\
\text { micro, or pico, or } \\
\text { femto }\end{array}$ & $\begin{array}{l}\text { Cell size can be } \\
\text { either macro, or } \\
\text { micro, or pico, or } \\
\text { femto }\end{array}$ & $\begin{array}{l}\text { Cell size can be } \\
\text { either macro, or } \\
\text { micro, or pico, or } \\
\text { femto }\end{array}$ & $\begin{array}{l}\text { Small cells such } \\
\text { as pico and femto }\end{array}$ \\
\hline $\begin{array}{l}\text { Band class } 14 \text { ra- } \\
\text { dio }\end{array}$ & Yes & Yes & Yes & Yes & Yes \\
\hline Standalone & Yes & No & No & Yes & No \\
\hline Availability & Immediate & $\begin{array}{l}\text { Vehicular drive } \\
\text { time }\end{array}$ & $\begin{array}{l}\text { Vehicular drive } \\
\text { time }\end{array}$ & $\begin{array}{l}\text { Vehicular drive } \\
\text { time and system } \\
\text { deployment time }\end{array}$ & $\begin{array}{l}\text { Aerial launch } \\
\text { time }\end{array}$ \\
\hline Power & $\begin{array}{l}\text { Limited to } \\
\text { vehicle bat- } \\
\text { tery }\end{array}$ & Generator & Generator & Generator & $\begin{array}{l}\text { Limited to the } \\
\text { airframe }\end{array}$ \\
\hline Deployment time & Zero to low & Low & Medium & Long & Long \\
\hline $\begin{array}{l}\text { Deployment na- } \\
\text { ture }\end{array}$ & $\begin{array}{l}\text { EFR vehi- } \\
\text { cles }\end{array}$ & $\begin{array}{l}\text { Dedicated } \\
\text { Trunks }\end{array}$ & $\begin{array}{l}\text { Dedicated Trail- } \\
\text { ers }\end{array}$ & $\begin{array}{l}\text { Dedicated Trucks } \\
\text { with Trailer }\end{array}$ & $\begin{array}{l}\text { Aerial such as } \\
\text { UAVs and bal- } \\
\text { loons }\end{array}$ \\
\hline $\begin{array}{l}\text { Deployment } \\
\text { quantity }\end{array}$ & Thousands & Hundreds & Hundreds & Dozens & $\begin{array}{l}\text { Dozens (based on } \\
\text { experimentations } \\
\text { and simulations) }\end{array}$ \\
\hline Incident duration & Low & Medium & Medium & Long & Long \\
\hline
\end{tabular}

FNPRM focuses on the overall architecture and proposes additional requirements to promote and enable nationwide interoperability among public safety broadband networks operating in the $700 \mathrm{MHz}$ band [74]. 


\subsubsection{FirstNet Architecture}

As per [13], Fig. 3.8 shows the proposed FirstNet architecture. The FirstNet is a LTE-based broadband network dedicated to public safety services. Core features of FirstNet will include direct communication mode, push-to-talk (PTT), full duplex voice system, group calls, talker identification, emergency alerting, and audio quality. Band class 14 (D Block and Public Safety Block) shown in Fig. 3.5 is the dedicated band and will be used by FirstNet PSN [98]. The FirstNet is composed of distributed core, terrestrial mobile system, mobile satellite system, and deployable systems. Distributed core consists of an evolved packet core (EPC) network and a service delivery platform to provide various services to end users such as EFR. The terrestrial mobile system comprises terrestrial based communication, while mobile satellite system will use a satellite communication link to communicate with distributed core network for services. Deployable systems are cells on wheels, providing services in network congestion areas or filling coverage holes.

In [172], FirstNet vehicular PSNs were divided into 5 categories: vehicle network system (VNS), cells on light trucks (COLTS), cells on wheels (COW), system on wheels (SOW), and deployable aerial communications architecture (DACA). These different vehicular FirstNet PSN systems are expected to play a significant role in providing coverage extension for NPSBN deployment. Thus, these deployments will deliver greater coverage, capacity, connectivity, and flexibility in regions which are outside of terrestrial coverage, or where traditional coverage become unavailable due to a natural or man-made disaster $[61,65]$. Table 3.4 discusses the characteristics of the five different vehicular PSN architectures in FirstNet.

As per [13], FirstNet broadly defines LTE network in distinct layers: Core network, transport backhaul, radio access network (RAN) and public safety devices as seen in Fig. 3.9. Public safety devices can be smartphones, laptops, tablets or any 


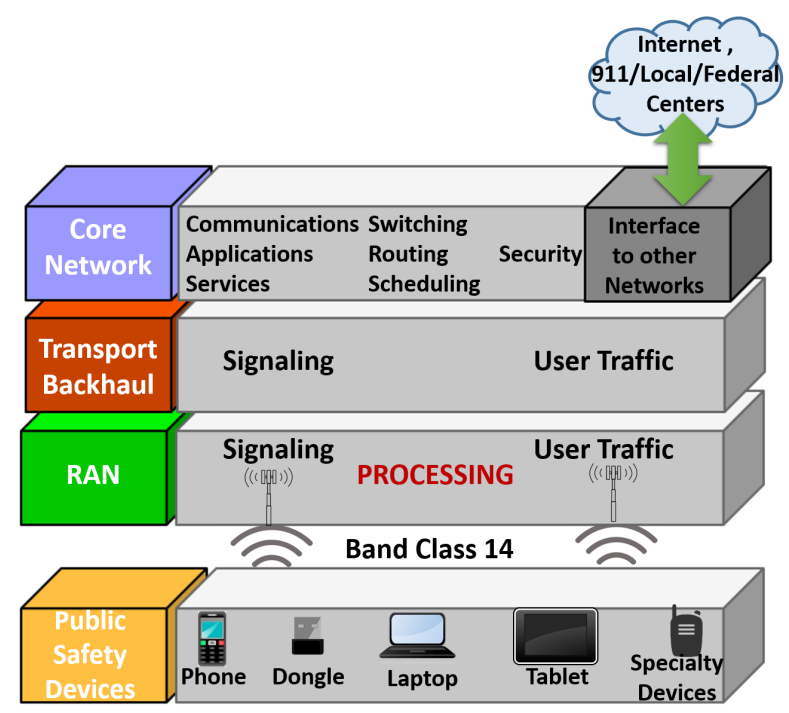

Figure 3.9: LTE band class 14 architecture for FirstNet [13].

other LTE-based user equipment. RAN implements radio access technology, which conceptually resides between public safety devices and provides a connection to its $\mathrm{CN}$. The transport backhaul comprises intermediate CN and subnetworks, wherein $\mathrm{CN}$ is the central part of a telecommunication network that provides various services to public safety devices across access network.

The band class 14 communication will be based on the LTE commercial standards and is solely dedicated to PSC in North America region. Table 3.5 shows the available downlink and uplink frequencies and the E-UTRA Absolute Radio Frequency Channel Number (Earfcn) for a band class 14 system. As per 3GPP specifications, the carrier frequency is specified by an absolute radio-frequency channel number (ARFCN). The Earfen is a designated code pair for physical radio carrier for both transmission and reception of the LTE system i.e., one Earfcn for the uplink and one for the downlink. The Earfcn also reflects the center frequency of an LTE carriers, and falls within range of 0 to 65535 . The bandwidth allocated for band class 14 is $10 \mathrm{MHz}$. 
Table 3.5: Band class 14 parameters.

\begin{tabular}{|l|l|l|l|l|l|l|}
\hline & \multicolumn{3}{|c|}{ Downlink (MHz) } & \multicolumn{3}{c|}{ Uplink (MHz) } \\
\hline & Low & Middle & High & Low & Middle & High \\
\hline Frequency & 758 & 763 & 768 & 788 & 793 & 798 \\
\hline Earfcn & 5280 & 5330 & 5379 & 23280 & 23330 & 23379 \\
\hline
\end{tabular}

\subsection{LMRS and LTE Convergence}

Since data is just as important as voice in PSC scenario, mission-critical voice and data communication together can deliver the much required reliable intelligence and support to EFR during any emergency [119]. For example, in the United States, FCC has been taking steps to achieve this LMRS-LTE convergence by setting various policies and a framework for a NPSBN. A private network like NPSBN would include FirstNet (a single licensed operator), $700 \mathrm{MHz}$ band class 14 (single frequency band), and LTE (a common operating technology). With large investment in LMRS over the last decade or so, LMRS has gained a national footprint in the United States [75]. During this time the United States has relied on LMRS technology to provide mission critical communication to the EFR. LTE deployments for replacing $2 \mathrm{G}$ and $3 \mathrm{G}$ infrastructure had no issues in relative measures, whereas the integration of LTE with LMRS is going to be a challenging task. As the complete roll out of the NPSBN by FirstNet would take few years to meet all mission-critical EFR requirements, it is inevitable for LMRS and LTE to coexist for some time in the near future.

There are many deployment models available today for converging LMRS and LTE in PSC. This includes the combination of mission-critical radios on PSN, broadband devices on dedicated broadband networks, and consumer grade devices on commercial carriers. The major challenges would be 1) providing an efficient end-user experience to EFR using a multitude of different device, networks, and infrastructure 


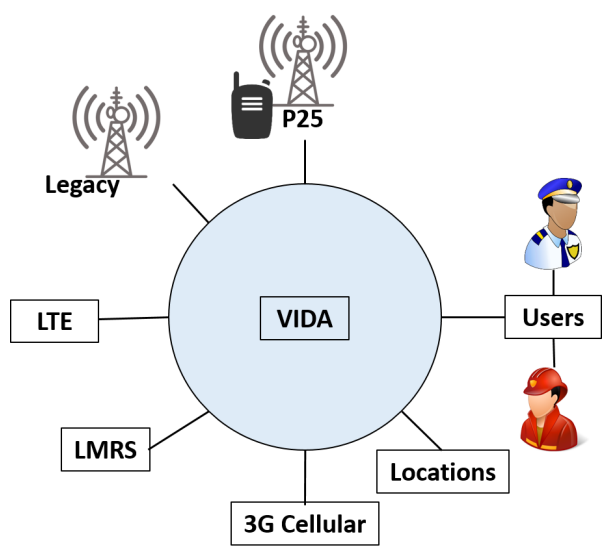

Figure 3.10: VIDA from Harris Corporation is a converge platform that integrates legacy and broadband PSC into a core network [14].

and 2) sharing mission-critical information regardless of device or network, without compromising security.

As a step towards the next generation public safety networks, Motorola Solutions has envisioned LMRS taking advantage of public and private LTE broadband networks. APX7000L, as shown in Fig. 6(b), is a portable APCO-25 digital radio converged with LTE data capabilities in a public-safety-grade LMR handheld device [2]. In order to have a seamless transition between LMRS and broadband networks, Motorola VALR mission critical architecture is utilized [120]. Harris Corporation's VIDA mission-critical platform provides an ability to support narrowband and broadband PSC technologies [14] as illustrated in Fig. 3.10. Similarly, Etherstack's LTE25 solution bridges LTE with existing APCO-25 narrowband networks with integrated push-to-talk solutions and interoperability in multi-vendor APCO25/LTE networks [244]. These developments build flexibly on technological advances, without compromising requirements, security, and coverage need for missioncritical communication. The converged solutions and frameworks have a common goal to provide mission-critical solutions, location services, and priority/emergency call support. 
These devices, solutions, and frameworks would provide EFR, the most need continuous connection to mission-critical voice, and data communications, and would be a first step in providing a necessary infrastructure for NPSBN. The LMRS and LTE both have individual strengths, and their convergence would result in a nationwide PSN and therefore enable a better collaboration amongst EFR, interoperability, data capability, security, robust coverage, and provide mission critical communications.

\subsection{Mission-Critical PTT over LTE}

The main goal of mission-critical voice is to enable reliable communication between various EFR. The essential attributes of mission -critical voice is to enable fast-call setup and group communication amongst the EFR, push-to-talk for various group talks, high audio quality, emergency alerting, and support secure/encrypted voice communication. The mission -critical encryption uses data encryption standard (DES) and advanced encryption standard (AES) for over-the-air-rekeying application $[108,113]$. To support mission-critical voice, LTE needs to incorporate all the essential attributes mentioned in this section. The objective of mission-critical PTT over LTE would be to emulate the functions by LMRS.

The 3GPP group is actively working to produce technical enhancement for LTE that support public safety applications. The main objective of $3 \mathrm{GPP}$ is to protect the quality of LTE while including the features needed for public safety. In LTE Release 12, 3GPP has focused on two main areas to address public safety applications. These focus areas include proximity services (ProSe) that enable optimized communication between the mobiles in physical proximity and group call system that support operations such as one-to-many calling and dispatcher services which enable efficient and dynamic group communication [15]. 
Path (a) in Fig. 3.11 shows the current conventional LTE communication whereas a network assisted discovery of users in close physical proximity is shown in Fig. 3.11 path (c). The direct communication, between these users with or without the supervision of the network is illustrated in Fig. 3.11 as path (b). In direct communication a radio can establish a communication link between the mobile users without going through a network, thus saving network resources and also enable mission-critical communication amongst the EFR even outside the network coverage $[15,111,112]$.

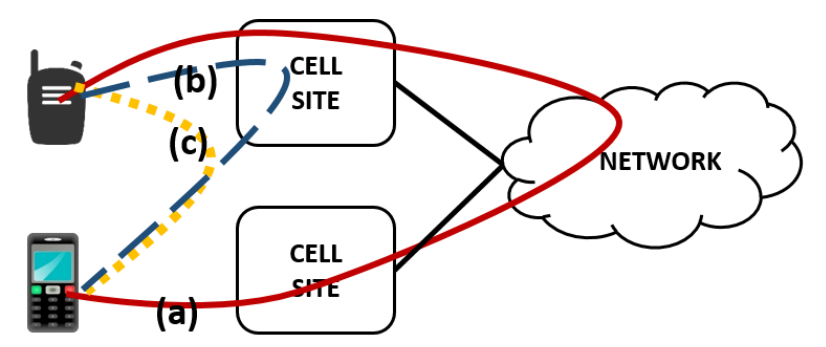

Figure 3.11: Proximity service examples as proposed in 3GPP LTE Release 12[15]. Path (a) shows current conventional LTE communication path, (b) shows direct communication with proximity services and (c) shows locally routed communication with proximity services.

The requirement definitions of mission-critical PTT over LTE are ongoing and will be finalized in LTE Release 13, which will enhance the D2D/ProSe framework standardized in Release 12 and support more advanced ProSe for public safety applications $[110,117,118]$. Release 12 added basic discovery and group communications functionality specific to D2D/ProSe. ProSe direct discovery, ProSe UE-to-network relay, ProSe UE-to-UE relay, and group communication among members via network, and a ProSe UE-to-network relay for public safety application will be included as part of Release 13 [117]. Isolated E-UTRAN operation for public safety is an another public safety feature included in Release 13 specifications. It would support locally routed communications in E-UTRAN for nomadic eNodeBs operating without a backhaul for critical communication [117], as shown in Fig. 3.11. 


\subsection{Comparison of LMRS and LTE PSC}

The LMRS technology has been widely used in PSC for over a decade with significant technological advancements. On the other hand, with the emerging of the LTE push-to-talk technology, focus has been shifting towards high speed low latency LTE technology. With various advancements in commercial networks, it can be another possible viable solution for PSC. Consider an emergency scenario, where different public safety organization using different communications technologies arrive at the incident scene. As shown in Fig. 1.1, these public safety organizations can be using different types of PSNs. In particular, the PSN deployed in the United States is a mixture of LMRS, FirstNet, and commercial networks. The LMRS provides push-to-talk mission critical voice capability over narrowband, with restricted data rates for voice/data services. However, LMRS has a wide area coverage, which can be achieved through the deployment of high-power base stations and handsets, portable base stations, repeaters, and/or peer-to-peer communication [107]. FirstNet provides high-speed network and can transform EFR capabilities with real-time updates. EFR can still rely on LMRS for mission-critical voice communication alongside FirstNet for high-speed data.

\subsubsection{Network Architecture}

Commercial networks are IP-based technologies such as $2 \mathrm{G} / 3 \mathrm{G} / 4 \mathrm{G} / \mathrm{Wi}-\mathrm{Fi}$. These technologies need adequate signal coverage and network scaling to support PSC services between EFR and victims. Due to existing commercial infrastructure, these networks are beneficial for providing PSC services. However, commercial networks are vulnerable to network congestion due to higher intensity of inbound and outbound communication at the incident scene by the public safety organizations and 


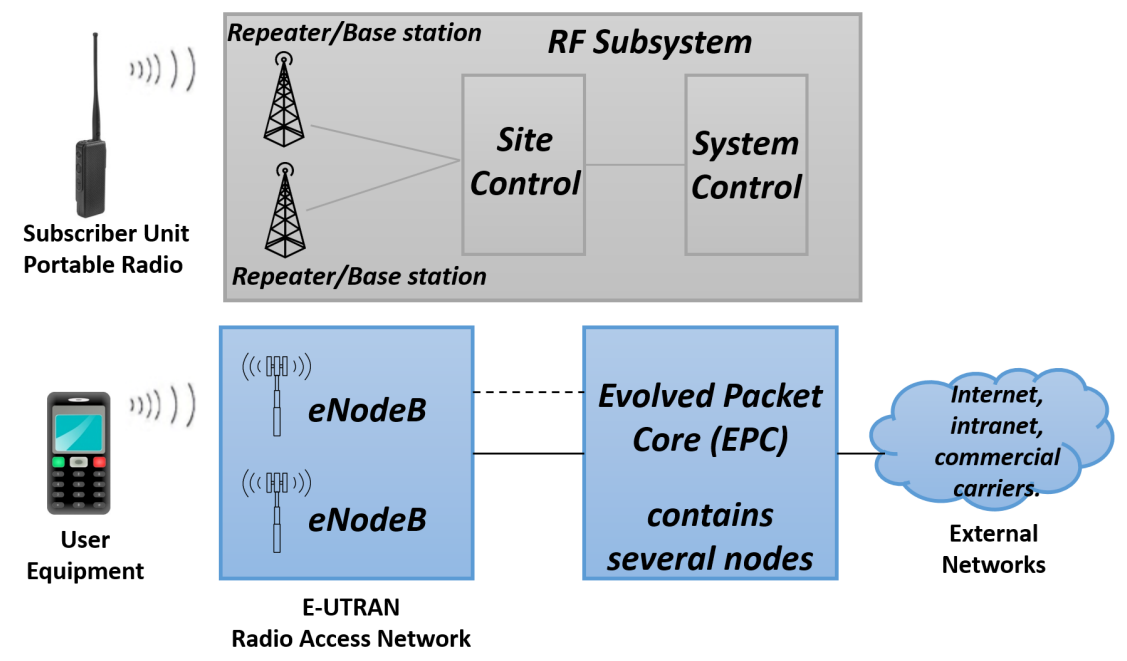

Figure 3.12: System level representation of the LMRS and the LTE networks for PSC.

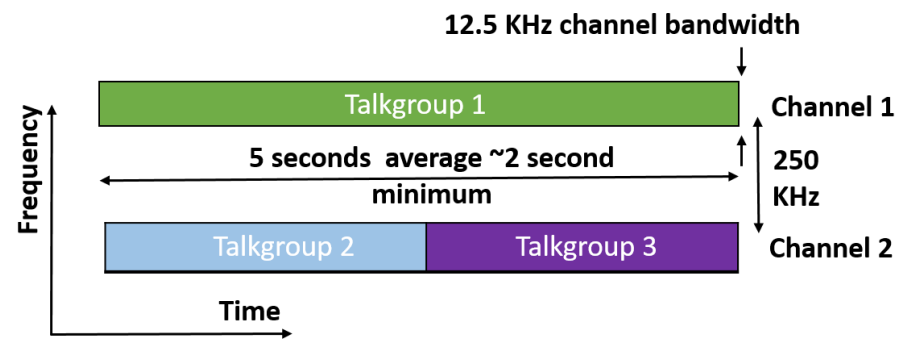

Figure 3.13: APCO-25 channel configuration.

victims. Such vulnerability can result in disrupted communications services and bring harm to victims.

System level block representation of the LMRS and LTE system are as shown in Fig. 3.12. The LMRS comprises of a mobile/portable subscriber units, repeater or a base station which is connected to an RF subsystem and then to a customer enterprise network. On other hand the LTE system includes user equipment (UE), eNodeB, and EPC which is connected to external Network. EPC, also known as system architecture evolution (SAE), may also contain several nodes. Key components of EPC are mobility management entity (MME), serving gateway (SGW), public data network gateway (PGW), and policy and charging rules function (PCRF). 


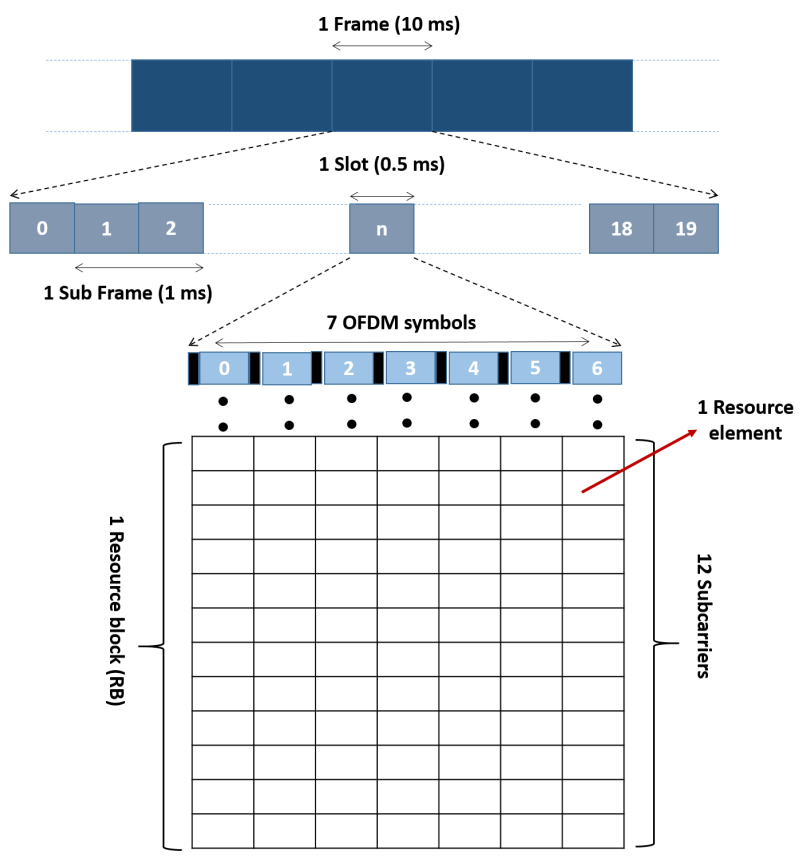

(a) LTE frame structure.

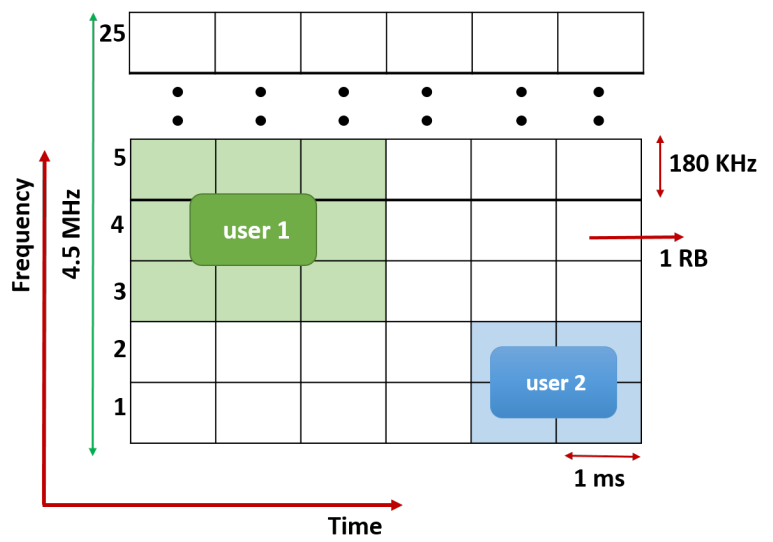

(b) LTE channel configuration. Each block represents a $\mathrm{RB}$, an UE can be scheduled in one or more RBs. LTE FirstNet gives more flexibility in scheduling users to their best channels, and hence enables better QoS.

Figure 3.14: LTE channel configuration.

\subsubsection{Channel Configuration and Frame Structure}

Channel configuration for the LMRS is shown in Fig. 3.13. In an LMRS the supported bandwidth are $6.25 \mathrm{KHz}, 12.5 \mathrm{KHz}$, and $30 \mathrm{KHz}$, wherein $12.5 \mathrm{KHz}$ is the 
standard bandwidth. The separation between two channels is usually $250 \mathrm{KHz}$ or higher. The talkgroups channel assigned during a transmission can last for a duration between 2 to 5 seconds. The data channel has bandwidth of $12.5 \mathrm{KHz}$ and is FDMA and/or TDMA channel. The data channel is designed with an aim to provide functionality like over the air rekeying, mission-critical voice etc,. On the other hand, the control channel is used solely for resource control. The main task for control channel is to allocate resources, digital communication message bearer, and message handler between RF subsystem and SU. The APCO-25 control channel uses C4FM modulation scheme and supports a baud rate of 9600 bits/s [96].

The LTE frame structure as seen in Fig. 3.14a, has one radio frame of $10 \mathrm{~ms}$ duration, 1 sub-frame $1 \mathrm{~ms}$ duration, and 1 slot of $0.5 \mathrm{~ms}$ duration. Each slot comprises of 7 OFDM symbols. The LTE UL and DL transmission is scheduled by resource blocks (RB). Each resource block is one slot of duration $0.5 \mathrm{~ms}$, or $180 \mathrm{KHz}$, and composed of 12 sub-carriers. Furthermore, one RB is made up of one slot in the time domain and 12 sub-carriers in the frequency domain. The smallest defined unit is a resource element, which consists of one OFDM subcarrier. Each UE can be scheduled in one or more RBs as shown in Fig. 3.14b. The control channel in LTE is provided to support efficient data transmission, and convey physical layer messages. LTE is provided with three DL physical control channels, i.e., physical control format indicator channel, physical HARQ indicator channel, and physical downlink control channel. Physical uplink control channel is used by UE in UL transmission to transmit necessary control signals only in subframes in which UE has not been allocated any RBs for the physical uplink shared channel transmission. Physical downlink shared channel, physical broadcast channel, and physical multicast channel are the DL data channels, whereas physical uplink shared channel and physical random access channel are the UL data channels. 
Table 3.6: Comparison between the LMRS and the LTE system.

\begin{tabular}{|c|c|c|}
\hline Parameters & LMRS & LTE \\
\hline Applications & $\begin{array}{l}\text { Mission-critical voice, data, location } \\
\text { services, and text. }\end{array}$ & $\begin{array}{l}\text { Multimedia, location services, text, and real-time } \\
\text { voice and data. }\end{array}$ \\
\hline Data Rates & $\begin{array}{l}\text { APCO- } 25 \text { has a fixed data rate of } 4.4 \\
\text { Kbits/s for voice communications and } \\
9.6 \text { to } 96 \text { Kbits/s for data only system. }\end{array}$ & $\begin{array}{l}\text { Depends on various factors such as signal-to- } \\
\text { interference-plus-noise-ratio (SINR), bandwidth allo- } \\
\text { cation, symbol modulation, forward error correction } \\
\text { code, and number of UEs attached to the eNodeB. As } \\
\text { per } 3 \mathrm{GPP} \text { specifications, LTE-Advanced provides an } \\
\text { increased peak data rate of downlink (DL) } 3 \mathrm{Gbits} / \mathrm{s} \text {, } \\
\text { and } 1.5 \mathrm{Gbits} / \mathrm{s} \text { in the uplink (UL). }\end{array}$ \\
\hline SINR & $\begin{array}{l}\text { DAQ is Delivered Audio Quality rep- } \\
\text { resenting the signal quality for digital } \\
\text { radios. The DAQ scale ranges from } 1 \\
\text { to } 5 \text {, with } 1 \text { being unintelligible to } 5 \\
\text { being perfect [90]. Public safety radio } \\
\text { uses a DAQ of } 3.4 \text {, which corresponds } \\
\text { to speech that is understandable and } \\
\text { rarely needs repetition, i.e., SINR of } \\
17.7 \mathrm{~dB} \text {. }\end{array}$ & $\begin{array}{l}\text { Does not depend on cell load but relies on factors such } \\
\text { as channel quality indicator (CQI) feedback, trans- } \\
\text { mitted by the UE. CQI feedback lets eNodeB se- } \\
\text { lect between QPSK, 16QAM, and 64QAM modula- } \\
\text { tion schemes and a wide range of code rates. A SINR } \\
\text { greater than } 20 \mathrm{~dB} \text { denotes excellent signal quality } \\
\text { (UE is closer to eNodeB), whereas SINR less than } 0 \\
\text { dB indicates poor link quality (UE is located at cell } \\
\text { edge). }\end{array}$ \\
\hline $\begin{array}{l}\text { Modulation } \\
\text { type }\end{array}$ & $\begin{array}{l}\text { Fixed modulation scheme with } 2 \text { bits } \\
\text { per symbol. }\end{array}$ & $\begin{array}{l}\text { Based on CQI feedback eNodeB can select between } \\
\text { QPSK, 16QAM, and 64QAM modulation scheme } \\
\text { where higher modulation scheme needs higher SINR } \\
\text { value. }\end{array}$ \\
\hline $\begin{array}{l}\text { Forward } \\
\text { Error Correc- } \\
\text { tion (FEC) }\end{array}$ & FEC rate is fixed as per DAQ3.4. & $\begin{array}{l}\text { FEC rate is variable and dependent on the SINR val- } \\
\text { ues. Higher rate codes (less redundant bits added) can } \\
\text { be used when SINR is high. }\end{array}$ \\
\hline $\begin{array}{l}\text { Antenna } \\
\text { Configura- } \\
\text { tion }\end{array}$ & $\begin{array}{l}\text { Single input single output (SISO) node } \\
\text { with an omni-directional pattern. }\end{array}$ & $\begin{array}{l}\text { MIMO has been an integral part of LTE with a goal } \\
\text { of improving data throughput and spectral efficiency. } \\
\text { LTE-Advanced introduced } 8 \times 8 \text { MIMO in the DL and } \\
4 \times 4 \text { in the UL. }\end{array}$ \\
\hline $\begin{array}{l}\text { Transmission } \\
\text { Power }\end{array}$ & $\begin{array}{l}\text { subscriber unit always transmits at full } \\
\text { power. As per [2] Subscriber unit } \\
\text { transmit power is within range set of } \\
1-5 \text { Watts and for base station out- } \\
\text { put power } 100-125 \mathrm{Watts} \text { depending } \\
\text { on } 700 \mathrm{MHz}, 800 \mathrm{MHz}, \mathrm{VHF} \text {, or UHF } \\
\text { frequency. }\end{array}$ & $\begin{array}{l}\text { UE's transmission power can be controlled with a } \\
\text { granularity of } 1 \mathrm{~dB} \text { within the range set of }-40 \mathrm{dBm} \text { to } \\
+23 \mathrm{dBm} \text { (corresponding to maximum power trans- } \\
\text { mission power of } 0.2 \text { Watts). As per } 3 \mathrm{GPP} \text { Release } \\
9 \text { notes, maximum transmission power is } 46 \mathrm{dBm} \text { for } \\
\text { micro base station and } 24 \mathrm{dBm} \text { for pico base station. }\end{array}$ \\
\hline Cell range & $\begin{array}{l}\text { Based on the antenna height above } \\
\text { the ground the maximum transmission } \\
\text { range can be up to } 43 \mathrm{~km}[91] .\end{array}$ & $\begin{array}{l}\text { In theory, GSM base station can cover an area with a } \\
\text { radius of } 35 \mathrm{~km} \text {. In case of dense urban environment, } \\
\text { coverage can drop to area with radius between } 3 \mathrm{~km} \text { to } \\
5 \mathrm{~km} \text { and less than } 15 \mathrm{~km} \text { in dense rural environment } \\
\text { [245]. Small cells such as microcell, picocell, femtocell } \\
\text { have even smaller range and cover up to } 2 \mathrm{~km}, 200 \mathrm{~m} \text {, } \\
\text { and } 10 \mathrm{~m} \text { respectively. }\end{array}$ \\
\hline $\begin{array}{l}\text { Multicast } \\
\text { and Unicast }\end{array}$ & $\begin{array}{l}\text { Multicast for group voice calls and uni- } \\
\text { cast for data. }\end{array}$ & $\begin{array}{l}\text { Primarily unicast for data, MBMS can support multi- } \\
\text { cast data. }\end{array}$ \\
\hline Security & 256-bit AES encryption $[246,247]$. & Standardized using 128-bit key [109]. \\
\hline
\end{tabular}


Some further differences between the LMRS and the LTE networks are summarized in Table 3.6 [246].

\subsection{Emerging Technologies for PSC}

LTE technology has been standardized by the 3GPP in 2008, and has been evolving since then. In response to growing commercial market demands, LTE-Advanced was specified as 3GPP Release 10. 3GPP has developed Release 11 and Release 12 with the aim of extending the functionality and raise the performance of LTEAdvanced [248]. With a vision of 2020, currently LTE has become the target platform for machine-to-machine communications, PSC, and device-to-device services [249]. Since the deployment of LTE, 4G is reaching its maturity and relatively incremental improvements are to be expected. The 5G technology is expected to have fundamental technological components that will transform the capabilities of broadband networks [250]. For example, full-fledged efforts from researchers at the University of Surrey's 5G Innovation Centre managed to attain one terabit per sec-

ond (Tbps) of data speed [251]. The three big technologies described in [250] that will be shaping $5 \mathrm{G}$ are ultra-densification of base station deployments, millimeter wave (mmWave) communications, and massive MIMO.

This section of paper is an attempt to emphasize the potential of the technological advancement achieved through LTE, LTE-Advanced, and 5G for transforming the PSC capabilities. In particular, use of eMBMS, millimeter wave, massive MIMO techniques, small cells, unmanned aerial vehicles (UAVs), LTE-based V2X, unlicensed operation of LTE in the context of PSC, cognitive radio, wireless sensor networks, internet of things, and cybersecurity for PSN will be reviewed. 


\subsubsection{Multimedia Broadcast Multicast Service (MBMS)}

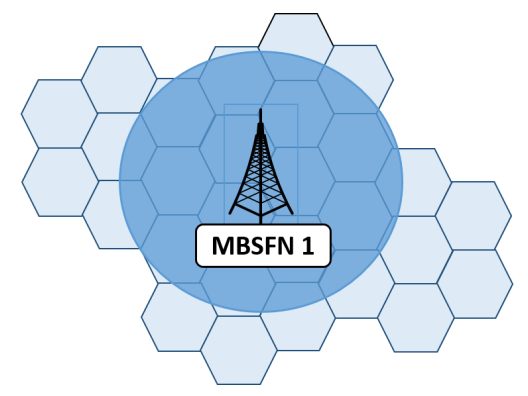

Figure 3.15: In a single frequency network, base stations transmit the same signal at the same time and over the same frequency channel to UE. In this example MBSFN area, the group of cells perform synchronized eMBMS transmission. These transmitted signals appear as multipath components to the UE.

The MBMS is a point-to-point interface specification for upcoming 3GPP cellular networks $[130,133,134]$. The MBMS is designed to provide efficient delivery of broadcast and multicast services with a cell and core network. Broadcast transmission across multiple cells is via single-frequency network configuration. The MBMS feature can be divided into the MBMS Bearer Service and the MBMS User Service $[133,134]$. Support for evolved MBMS (eMBMS) will be also included in Release 13, which has important applications for PSC. The eMBMS is designed to improve the efficiency of legacy MBMS. The LTE technology is backward compatible with legacy $3 \mathrm{G}$ MBMS and supports new features of the broadcast networks such as digital video broadcast-terrestrial networks.

The eMBMS is a point-to-multipoint interface where multiple LTE cells can be grouped into Multicast/Broadcast Single Frequency Network (MBSFN) and all of the cells in an MBSFN will simulcast the same information for each bearer as shown in Fig. 3.15. The eMBMS broadcast bearers provide advantages of unlimited group sizes within a cell, better cell edge performance due to simulcast, and is independent of the talkgroups size. The eMBMS would provide PSC services with 
an ability to use a single set of resources for the traffic destined to multiple public safety devices. The public safety eMBMS broadcast applications would be group calls, PTT, real-time emergency video broadcast, surveillance video broadcast, and non-real-time multimedia services. The eMBMS broadcast coverage improvement would be similar to that of LMRS simulcast[131, 132].

\subsubsection{Millimeter Wave (mmWave)}

The severe spectrum shortage in conventional cellular bands has attracted attention towards mmWave frequencies that occupy the frequency spectrum for $30 \mathrm{GHz}$ to $300 \mathrm{GHz}$. It is also considered as a possible candidate from next-generation smallcellular networks $[135,142]$. The availability of huge bandwidth in the mmWave spectrum can alleviate the concerns of wireless traffic congestion which is observed in conventional cellular networks $[139,252]$. The mmWave transceivers can cover up to about a kilometer communication range in some scenarios [252]. Moreover, densely laid out mmWave small cells in congested areas can expand the data capacity and provide a backhaul alternative to cable [136]. The mmWave's ability opens up possibilities for new indoor and outdoor wireless services [135].

However, the key limitation of the mmWave technology is its limited range, due to the excessively absorbed or scattered signal by the atmosphere, rain, and vegetation. This limitation causes the mmWave signals to suffer very high signal attenuation, and to have reduced transmission distance. The researchers and scientists have overcome this loss with good receiver sensitivity, high transmit power, beamforming, and high antenna gains [135-138, 141, 142, 252, 253].

A possible solution for extending the mmWave range is proposed in [140]. Due to limited propagation range of mmWave, a reliable communication over mmWave 
requires a substantial number of access points. The authors discuss the transmission of radio frequency signals between the mmWave small cell base stations and radio access points using optical fibers to increase the coverage. This radio over fiber communication is possible by applying advanced optical upconversion techniques. In [141], various beamforming techniques are introduced for indoor mmWave communication.

A well augmented mmWave system can position itself as a reasonable solution for PSC needs, by addressing the concerns and requirements such as network congestion and high data rates. A densely populated mmWave small-cell networks can also address the coverage needs during emergency situations.

\subsubsection{Massive MIMO}

MIMO is an antenna technology that can be used to reduce communication errors and scale the capacity of a radio link using multiple transmit and receive antennas. Recently massive MIMO is an emerging technology that uses large number of service antennas when compared to current MIMO technology [148]. Since the introduction of massive MIMO in [144], some researchers have shifted focus on solving related traditional problems [143, 145-148].

Massive Massive MIMO is typically considered in time-division duplex systems to exploit channel reciprocity since it eliminates the need for channel feedback [147, 254]. However, the massive MIMO system is limited by pilot contamination, which is an impairment that is observed during channel estimation stage in TDD networks. The pilot contamination is caused due to the non-orthogonal nature transmission of pilots from neighboring cells $[147,254]$. Nevertheless, the effects of pilot 
contamination can be reduced by using mitigation techniques such as pilot-based and subspace-based estimation approaches [147].

Overall, massive MIMO can provide benefits such as $100 \times$ radiated energy efficiency, $10 \times$ capacity increase over traditional MIMO, extensive use of inexpensive low-power components, reduced latency, simplification of the medium access control, spectrum efficiency, reliability, and robustness [146-148].

Massive MIMO can be used for achieving high throughput communications in an emergency situation. For example, real-time situational awareness via high definition multimedia can be enabled via massive MIMO techniques. Massive MIMO can also provide better penetration into buildings due to directional transmissions. This ability of deep penetration can be utilized by the EFR during emergency scenario such as building fire.

\subsubsection{Small Cells}

Heterogeneous network (HetNet) topology blends macrocells, picocells, and femtocells. HetNet topology aims at providing uniform broadband experience to all users ubiquitously in the network $[149,152,153,155]$. Shrinking cell size benefits by reuse of spectrum across the geographical area and eventually reduction in number of users competing for resources at each base station. Small cells can be deployed both with standalone or within macro coverage, and they can be sparsely or densely distributed in indoor or outdoor environments[149]. They transmit at substantially lower power than macro cells and can be deployed to improve the capacity in hot-

pots, eliminate coverage holes, and ease the traffic load at the macro base station $[152]$. 


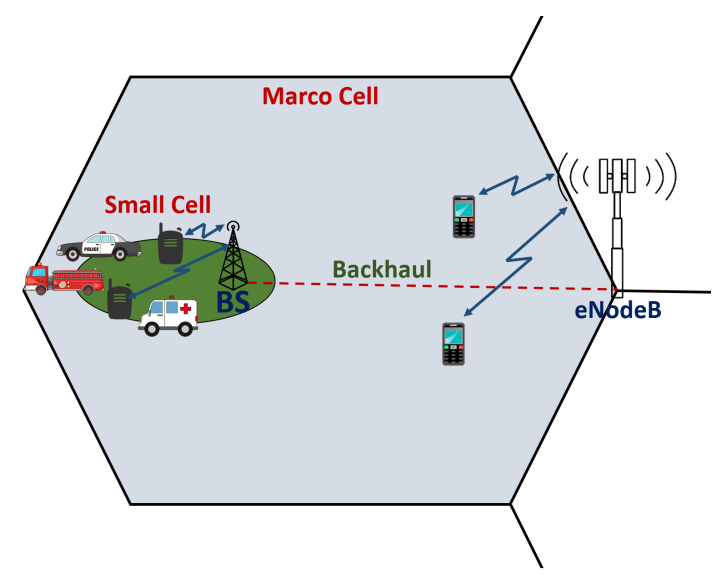

Figure 3.16: Small cell example for PSC during an emergency scene.

LTE based small cells can be deployed in an emergency scenario to improve PSC between EFR as shown in Fig. 3.16. This hotspot will improve the capacity of PSC, and as a result EFR do not have to compete with general public for eNodeB resources. The base stations used as small cells in such scenario can be portable/deployable systems. The backhual to these base stations can be via the closest eNodeB, via satellite link, or via its own dedicated backhaul. A flexible and low cost PSC deployment strategy using small cells can be effective and enhance the ability of the EFR to save lives.

The indoor LTE coverage is becoming a pressing concern as LTE deployments evolve. For instance, EFR would need communication coverage in various parts of a building such as the basement. However, building basements may have weak coverage since the existing LTE deployments does not always consider it crucial. This issue can be resolved using LTE mini-towers to boost indoor communication coverage. Nevertheless, indoor coverage remains ones of the pitfalls for LTE and needs to be addressed for an effective broadband PSN [255]. 


\subsubsection{Unmanned Aerial Vehicles (UAVs)}

In the United States, Hurricane Sandy affected 24 states, and particularly severe damage was observed in NJ and NY. Electrical power, terrestrial communication systems, and utilities cease to exist due to devastation caused by the Hurricane Sandy. Along with disruption of PSC and commercial wireless services, high winds caused damage to point-to-point backhaul links and fiber-optic cables were cut. The service disruption was observed over at least 3-5 days before any services began to restore. Hurricane Sandy exposed flaw in public-safety LTE plan as discussed in [256], due to loss of commercial cell sites and no backup coverage from commercial LTE carriers.

The deployment of LTE-equipped UAVs in such an emergency scenario would provide necessary voice and data coverage $[150,159,162]$. In [157], a similar use case is discussed where LTE-equipped UAVs is deployed as a relay node. UAV will share the same bandwidth allocated for public safety broadband network and PSN could also backhaul the traffic from UAV. The concept of using UAV from wireless communication has been a proven technology. UAV are easy to deploy and can quickly restore critical communications during an emergency operation. Alcatel-Lucent conducted a test with an ad-hoc LTE network using 4G smartphone and USB Modem. The UAV flew one kilometer while streaming 720p video [168]. Higher heights may occasionally result in line-of-sight and stronger connectivity. UAVs have limited battery powers, and therefore may have limited flight times, which may introduce operational constraints.

The use of UAVs in PSC networks pose several challenges that need to be re-

solved. For example, mobility model, topology formation, energy constraints, and cooperation between UAVs in a multi-UAV network are surveyed in [164]. Further- 
more, spectrum scarcity specific challenges and integration of cognitive radio into UAVs are addressed in [165].

UAVs can complement the capabilities of PSN and can operate in the same public safety spectrum block. They can also achieve a radio link with nearest functioning eNodeB [157]. They can be deployed in high-risk areas to assist EFR and used as a relay node to extend network coverage, as discussed in[158, 159, 169, 183]. With clearer rulemaking and policies on usage of UAVs in civilian space [156], UAVs can be inducted into PSN and making it a viable solution in filling coverage holes.

\subsubsection{LTE-based V2X Communication}

LTE-based V2X communication involves the communication of information from vehicle into devices of interest, and vice versa. The V2X communication has many applications such as navigation, driver assistance, travel information, congestion avoidance, and fleet management. It can occur in the form of vehicle to vehicle $(\mathrm{V} 2 \mathrm{~V})$, vehicle to infrastructure (V2I), and vehicle to pedestrian (V2P) communication [173-177]. The ProSe can find good application in V2X communication. In Release 13, the ProSe has been designed for pedestrian mobility speed, and further research and standardization is needed for extensions to be used directly for V2X $[177]$.

V2X communication can also find application in PSC. In an emergency situation Firefighters, police cars, ambulances vehicles can broadcast/multicast information to other traffic elements and pave way to the EFR vehicles and avoid any traffic congestion. The V2X communications can also be used during disaster management such as city evacuation. The vehicular ecological system can gather information such as accident points and traffic patterns from multiple sources. Furthermore, 
V2X communication can also broadcast this information to other vehicular nodes. The effective V2X communication can play critical role during disaster management and minimize loss to human life $[170,171]$.

\subsubsection{License Assisted Access (LAA)}

With the limited spectrum availability and ever increasing traffic demands, researcher and cellular companies are motivated to find complementary solutions to the licensed spectrum. Although the licensed spectrum is preferable to provide better user experience, exploring LTE in unlicensed spectrum can lead to higher spectral efficiency [184]. LTE in the unlicensed band (LTE-U) can complement the services provided by the LTE in licensed spectrum. As per the regulation, LTE signals transmitted in unlicensed spectrum must be accompanied by a licensed carrier. Primary carrier always uses licensed spectrum (either TDD or FDD) for both control signals and user signals. The secondary carrier would use the unlicensed spectrum for downlink only or both uplink and downlink [127, 178, 180, 181, 185]. $5 \mathrm{GHz}$ unlicensed spectrum is a top candidate for the operation that is globally available while it should be ensured that LTE-U design is forward-compatible with upcoming 3GPP specifications [178]. A robust design solutions for unlicensed bands, clearer

guidelines for operators, and coexistence framework would be necessary, if LTE and Wi-Fi have to coexist on fair share basis $[178,186,187,257]$.

LAA is an aggregation of LTE in the licensed and unlicensed spectrum. The main goal of LTE and LTE-Advanced for a licensed-assisted access to unlicensed spectrum would be to achieve better traffic offloading, achieve spectral efficiency, and provide a higher user experience in a cost-effective manner $[179,184]$. The Wi-Fi performance is maintained while coexisting with licensed-assisted access to 
unlicensed spectrum as discussed in $[181,183,257]$. The LAA benefits to all user, by increasing the capacity to support the indoor applications, providing seamless indoor/outdoor mobility, better coverage experience, efficient spectrum usage, and better network performance as outlined by the technical report [257]. However, operating on unlicensed spectrum may have design implications on LTE that are not yet well understood. In particular, co-existence between multiple LTE operators sharing the unlicensed band and co-existence with WLAN should be studied and addressed [188, 189].

PSC examples discussed earlier under Section 3.10.2 and Section 3.10.3 can benefit from licensed-assisted access to unlicensed spectrum. In Fig. 3.16, the small cell operating in unlicensed spectrum can be deployed outdoor and indoor. Extending the benefits of small cells with LTE-U would lead to better network performance, enhanced user experience by supplementing the downlink, and provide seamless mobility [154]. These benefits can enhance the EFR capabilities, provide much needed mission-critical communication, and improved data capacity to support real-time data/multimedia/voice [257]. In an emergency scenario, as discussed in Section IX.C where all the terrestrial infrastructure has been devastated. LTE-U based UAV operation can be considered [160, 161]. In [160], the feasibility of using UAV in unlicensed for the purpose of search and rescue operations of avalanche victims has been discussed. Based on the simulation results, it can be deduced that UAV operations in unlicensed spectrum can be used by EFR during search and rescue missions. 


\subsubsection{Spectrum sharing and cognitive radio}

With the explosion of various wireless technologies such as 5G, Internet of Things, home automation, and connected cars. The finite radio spectrum, must accommodate cellular calls and data traffic from various wireless devices. As a result, the radio spectrum is quickly becoming a scarce commodity. For example, according to FCC, United States will be soon running out of radio spectrum [68].

As the spectrum gets increasingly scarce, public safety agencies have to compete with commercial entities for the radio spectrum resources. If not properly addressed, problem of spectrum scarcity can result PSN to be less dependable in the future, and may face interoperability challenges [69, 70]. In [67], a group of experts from Australia and Europe provide opinions on recent research and policy options about spectrum management, including new approaches to valuation and sharing. Furthermore, effective detection and utilization of white spaces in PSC spectrum are crucial for minimizing outages and maximizing throughput [192]. In [190], pervasive spectrum sharing is envisioned as an enabler for PSNs with broadband communication capabilities.

Cognitive radio technology presents a viable solution to use spectrum more efficiently, which can potentially address the spectrum scarcity problem. A cognitive radio senses and learns the radio frequency spectrum in order to operate in unused portions of licensed spectrum or whitespaces without causing interference to primary licensed users $[66,191,194]$. Furthermore, a cognitive radio network offers support for heterogeneity, reconfigurability, self-organization, and interoperability with existing networks as discussed in [191, 195]. These ability of cognitive ra-

dio can be instrumental during disaster scenarios, with partially/fully destroyed networks. Moreover, the convergence of cognitive radio technology and device-to- 
device communication can improve the spectrum utilization [193] and also benefit PSC by mitigating spectrum scarcity.

\subsubsection{Wireless Sensor Networks}

A sensor network is composed of spatially distributed autonomous sensors with the ability to monitor and control physical environment. A typical sensor network has many sensor nodes and a gateway sensor node. Modern day sensor networks are usually bi-directional which cooperatively pass their data through the networks to the main location. Lately, sensor network received extensive interest for their use cases in PSN [196-198].

A sensor network is composed of spatially distributed autonomous sensors with the ability to monitor and control physical environment. A typical sensor network has many sensor nodes and a gateway sensor node. Modern day sensor networks are usually bi-directional which cooperatively pass their data through the networks to the main location. Lately, sensor networks received extensive interest for their use cases in PSN [196-198].

Wireless sensor networks (WSN) have the ability for situational awareness of a large physical environment, and this ability can support the decision makers with early detection of a possible catastrophic event [196]. Furthermore, the localization algorithms can provide key support for many location-aware protocols and enhance the situational awareness [199]. In a large-scale WSN deployment for situational awareness, the WSN is vulnerable to traffic patterns and node convergence as discussed in [200]. Further analyzes present the sink mobility as a potential solution to enhance the network performance in large-scale WSN with varying traffic patterns. 
A traditional multi-hop WSN is resources-constrained and uses fixed spectrum allocation. Therefore, by equipping a sensor node with cognitive radio capabilities, a WSN can reap the benefits of dynamic spectrum access as discussed in [198]. Furthermore, applying channel bonding schemes to cognitive-WSNs can result in better bandwidth utilization and higher channel capacity as surveyed in [201].

The deployment of large-scale WSNs into PSN, alongside the LTE-based PSN, have important advantages. With sensor gateway node connected to LTE-based PSN, data can seamlessly flow into public safety data centers. This real-time data can raise the situational awareness of the public safety agencies and reduces the EFR response time.

However, the integration of WSN into IP-based network makes it vulnerable to various threats as discussed in Section IX-K. The various security aspects of WSN in PSN are discussed in $[196,197]$.

\subsubsection{Internet of Things (IoT)}

The IoT revolves around the pervasive presence of objects such as radio-frequency identification tags, sensors, actuators, and mobile phones having machine-to-machine (M2M) communication either through a virtual or instantaneous connection. The IoT infrastructure is built using cloud computing services and networks of datagathering sensors. The IoT offers an ability to measure, infer, and understand the surrounding environment and delicate ecologies [205-207]. Ubiquitous sensing enabled by the wireless sensor networks also cuts across PSC frontiers. The M2M and connected devices can collect real-time data from EFR devices, such as body-worn cameras, sensors attached to gun holsters, and magazine/baton/radio pouches [208]. This ecosystem of EFR's personal area network can send the real-time alert from 
these IoT devices to increase the situational awareness. With the availability of broadband services, such as LTE and WiFi, this real-time data can be uploaded and stored using cloud services. The real-time analysis of this data at crime centers using artificial intelligence and decision-making algorithms can be used to respond easily in any emergency situations [204]. Furthermore, measures can be taken to prevent a situation turning into an emergency [202, 203, 258].

The IoT can change the way EFR execute their missions, helping them to be more effective and responsive. The real-time tracking of EFR using IoT, can relay the right information to the right person at the right time. Furthermore, this information will enhance real-time decision making capabilities of EFR and avoid any potential disasters. However, as these devices become more integrated into cloud services, they are exposed to vulnerabilities that pose risk to public safety mission-critical data. The important major challenge involves managing the complex threats created by the IoT, while taking advantage of newly discovered capabilities of IoT to enhance PSC [259].

\subsubsection{Cybersecurity Enhancements and Data Analytics for PSNs}

The public safety data available from public safety agents such as IoT, sensor nodes, and specialized public safety devices can play a crucial role in disaster management. With the help of intelligent learning techniques, the available data can be analyzed to understand the dynamics of disaster prone areas and plan the rescue operation. Moreover, the real-time data flowing in from different public safety agents can be analyzed to predict and issue an early warning in case of natural or man-made disaster. This data analysis can enhance the capabilities of PSN. The importance of 
data analytics and related case studies using big data have been surveyed in article [213].

Furthermore, the public safety entities access public safety data such as medical records, site information, and other video and data information useful to disaster management and emergency response. Such critical information is transmitted in the form of voice, data, or multimedia using wireless laptops, handheld public safety devices, and mobile video cameras. However, this public safety data can be exploited by criminals with proper technology. Therefore, PSN infrastructure must protect the integrity of all the public safety data that is being accessed and transmitted. With extensive elevated network privileges given to EFR during an emergency scenario, having a reliable communication becomes of critical importance [209, 211, 212].

In [209], authors discuss various PSN security threats such as, protection against evesdropping, protection against corrupt information, securing network interfaces between network elements, protection against data exfiltration, and protection against denial of service attacks. Identity management of every public safety device and EFR accessing the critical information is an absolute necessity. As discussed in [209-211] identity management can be characterized as identification, authentication, and authorization associated with individuals, public safety devices, or public safety applications. There are several proven security methods/techniques for identification, authentication, and authorization. However, the public safety agencies need to develop the technical and policy requirements for use of security techniques in PSN by considering the scope and context of their applicability to PSC. 
Table 3.7: PDPR identified bands/ranges by international telecommunication union (ITU) [101].

\begin{tabular}{|l|l|l|}
\hline ITU Region 1 & $\begin{array}{l}\text { Europe (including Rus- } \\
\text { sia and Middle East) } \\
\text { and Africa }\end{array}$ & $\begin{array}{l}380-470 \mathrm{MHz} \quad(380-385 / 390- \\
395 \mathrm{MHz})\end{array}$ \\
\hline ITU Region 2 & Americas & $\begin{array}{l}746-806 \mathrm{MHz}, 806-869 \mathrm{MHz}, \\
4940-4990 \mathrm{MHz}\end{array}$ \\
\hline ITU Region 3 & Asia-Pacific & $\begin{array}{l}406.1-430 \mathrm{MHz}, 440-470 \mathrm{MHz}, \\
\end{array}$ \\
& & $\begin{array}{l}806-824 / 851-869 \mathrm{MHz}, 4940- \\
\end{array}$ \\
& $\mathrm{MHz} \mathrm{MHz}$, and 5850-5925 \\
\hline
\end{tabular}

\subsection{Public safety broadband deployments in other regions of the world.}

Broadband deployment for PSC has been grabbing the spotlight in different parts of the world. The global standards collaboration emergency communications task force is investigating standards for a globally coordinated approach for PSC. The world radio conference (WRC-15) held in Geneva in November 2015, provided a platform to address public protection and disaster relief (PPDR) requirements. The WRC-15 Resolution 646 is an international agreement between UN and ITU. This agreement encourages the public safety entities to use frequency range $694-894 \mathrm{MHz}$, when outlining a draft for nationwide broadband PSN for PPDR applications [102]. The public safety entities across different countries have different operational frequency range and spectrum requirements.

Table 3.7 shows the frequency allocation for PPDR in various parts of the world. The amount of spectrum needed for PSC by different EFR agencies and applications on a daily basis differs significantly. To enable spectrum harmonization for nationwide and cross-border operations would need interoperability between various EFR 
systems used for PPDR. As a result, having a dedicated broadband spectrum for PPDR has been one of the WRC-15 agenda items [260].

Europe: The ECC Report 199 [103] addresses the broadband spectrum in Europe for PSC networks, which considers the $400 \mathrm{MHz}$ and $700 \mathrm{MHz}$ frequency bands. The report also concludes that $10 \mathrm{MHz}$ of spectrum for the uplink and another $10 \mathrm{MHz}$ for the downlink would provide sufficient capacity to meet the core requirements of PSC.

United Kingdom: The public safety agencies in the United Kingdom use narrowband TETRA PSN for data and mission-critical voice services. Furthermore, the United Kingdom also plans on building emergency services mobile communications programme (ESMCP), also referred as the emergency service network (ESN), which will deliver future mobile communication for the country's emergency services using 4G LTE network [261]. The United Kingdom is preparing for an orderly transition from TETRA to LTE-based ESN by the year 2020 [262]. However, there is no spectrum available in $700 \mathrm{MHz}$ at-least until 2019. Therefore, the ESN will latch onto $800 \mathrm{MHz}$ to implement voice calls over LTE, PTT capabilities, and satellite backhaul in hard-to-reach areas [32].

Canada: Industry Canada has initiated a framework and policy for public safety broadband spectrum in the bands $758-763 \mathrm{MHz}$ and $788-793 \mathrm{MHz}$, i.e., band class 14 as illustrated in Fig. 3.5. Canadian public safety band plan in upper $700 \mathrm{MHz}$ is shown in [104] and it corresponds to FirstNet's band class 14. Recently, the EFR in the City of Calgary, have been testing the first public safety LTE network in Canada [263]. With the help of high-speed broadband capabilities Calgary Police Services were able to quickly access data, analyze the information, and securely multicast data to EFR. The Calgary public safety LTE network is composed of 
cellular sites and uses LTE public safety devices such as VML750 and LEX missioncritical handhelds [263].

Australia and China: Similarly, in countries like Australia and China, LTE technology is fast becoming a primary choice for professional digital trunking command and dispatching services. The main goal is to build a rapid, flexible, and effective wireless multimedia communications network, which can be used to improve PDPR applications [101, 264].

\subsection{Issues and Open Research Directions}

While the technologies for PSC are ever evolving, given the discovery-delivery gap, not all advanced aspects of the public safety technology are commercially available. In particular, a full-fledged deployment of mission-critical LTE-based PSN is unattainable in the short term. This has resulted in the amalgamation of legacy and emerging public safety technologies. The convergence of LMRS-LTE public safety technologies can provide mission-critical voice and broadband data. However, designing and optimizing LMRS-LTE converged devices which can support mature 3GPP techniques such as MBMS is an open challenge for the engineers $[14,119]$.

The integration and optimization of 3GPP Release- 12/13 enhancements such as eMBMS, HetNets, LAA, and LTE-based V2X for PSC is an open challenge to both academic and engineering community. Currently, the MBSFN area and subframes in eMBMS are static and cannot be adjusted according to user distribution. Therefore, design and optimization of flexible MBSFN resource structures that can accommodate different user distributions have become a challenge and an important area of research. The integration of Release- 13 enhancements for D2D/ProSe framework into MBMS/eMBMS is an another open area of research [133]. Small 
cells need better assessment of attributes such as grid configuration, alignment with macro network to avoid interference, handover analysis, and backhauling to meet the capacity requirements of cell-edge users. Furthermore, a systematic convergence of mmWave, massive MIMO, and UAVs with small cells are an open areas of research $[149,152,155]$. LTE-U and LAA coexisting fairly in the unlicensed spectrum with other broadband technologies has become an intense debate. Therefore, having policies to ensure fair access to all technologies is increasingly becoming a popular area of investigation. Furthermore, designing protocols for carrier aggregation of licensed and unlicensed bands is an another important area of research [181, 257]. The integration of ProSe and LTE-based broadcast services into LTE-based V2X can ensure connectivity between EFR vehicles, roadside infrastructure, and people in PSN. This open area of research can help to further strengthen the mobility management, public warning systems, and disaster management. However, resource allocation, latency, interference management and mobility management are important issues in V2X communication that need some study [170, 171].

The mmWave, massive MIMO, and UAVs are advanced technologies with potential applications for PSC. Each of these technologies have their own metrics such as throughput provided, latency, the cost of providing higher throughput, ease of deployment, and commercial viability for assessing success in PSN. By addressing challenges such as interference management, spatial reuse, and modulation and coding schemes for dynamically changing channel user states, mmWave can result in more practical and affordable system for the development of cellular and PSN networks [136-139]. Similarly, efficient model of massive MIMO in PSN needs more accurate analysis of computational complexity, processing algorithms, synchronization of antenna units, and channel model [143]. The application of UAVs in PSN is shrouded by public's privacy concerns and lack of comprehensive policies, reg- 
ulations, and governance for UAVs [212]. Investigating the role of mmWave and massive MIMO with UAVs for higher throughput gains for robust UAV deployment [167]. Furthermore, developing new propagation model for better autonomy and quicker deployment of UAVS as aerial base stations is an open research direction $[150,157-163,166,183]$.

SDR and cognitive radio technologies provide additional flexibility and efficiency for overall spectrum use. These technologies can be combined or deployed autonomously for PSC. However, PSN using SDR or cognitive radio technologies must operate in compliance with radio and spectrum regulations. Furthermore, a comprehensive implementation of these technologies for PSC present technical and operational challenges. An SDR for PSC, which is interoperable across vendor infrastructures, frameworks, and radio bands is an area for future research [122-124]. Similarly, cognitive radio technology needs extensive research in areas of energy efficient spectrum sensing and sharing to be more effective. Extensive research over application of LAA, database-assisted spectrum sharing, and prioritized spectrum access in cognitive radio technology can further enhance the spectrum efficiency $[127,180,181,183]$.

Public safety wearables have become increasingly popular amongst EFR and are an important area of research. Tailoring public safety wearables and wireless sensors into IoT can help in real-time data aggregation and situation analysis. Therefore, WSNs and IoT needs to evolve within the wider context of PSN and address issues related to infrastructure, design, cost, interoperability, data aggregation, regulations, policies, and information security [196-198]. Furthermore constructing robust models for multi-hop synchronization and tethering real-time wireless sensor data attached to EFR equipment have become a contemporary area of research [202, 203]. 
The EFR access public safety data such as medical records, site information, multimedia, and other information useful for disaster management and emergency response. The perpetrators with proper technology can exploit this public safety data. Therefore, given the scope of next generation PSN, securing PSN infrastructures, public safety device integrity, and public safety data is an important issue and open area of research within cybersecurity space [204, 209, 211, 212]. Furthermore, the policy requirements for the use of public safety devices and information operating in PSN is an another major issue and area of research for the public safety agencies and federal regulatory bodies [210]. 


\section{CHAPTER 4}

\section{APCO-25 AND PUBLIC SAFETY LTE NS-3 SIMULATION}

The purpose of this chapter is to simulate LTE band class 14 system and APCO25 using NS-3 open source tool. The LTE band class 14 simulation is based on LENA LTE model [16] whereas LMRS simulation is based on the definition of APCO-25 portrayed in [95-97, 235]. We chose to use NS-3 simulator and LENA LTE model due to their effective characterization of LTE protocol details.

In order to be consistent and to provide a better comparative analysis between LMRS and LTE band class 14, we implement a single cell scenario, and similar user density. The main simulation goal is to compute aggregated throughput capacity and signal quality measurement with increasing cell size in case of both LTE and LMRS. Simulation setup, assumptions, and simulation parameters for LTE band class 14 system and LMRS are discussed in Section 4.2 and Section 4.1, respectively. Furthermore, we analyze these simulation results in Section 4.3 to strengthen the conclusion of the comparative study.

\subsection{NS-3 Simulation of LTE Band Class 14}

The simulation study of LTE band class 14 includes, throughput computation in up-

link and downlink, cell signal quality measurement in terms of SINR, and measuring signal quality in a inter-cell interference scenario. Fig. 4.1 shows the throughput simulation environment implemented in this chapter. Simulation environment consists of randomly placed multiple UEs and one eNodeB (eNB). Other environment entity includes remote host which provides service to end user. Remote host will provide IP-based services to UEs and user datagram protocol (UDP) application is installed on both the remote host and the UEs to send and receive a burst of packets. 


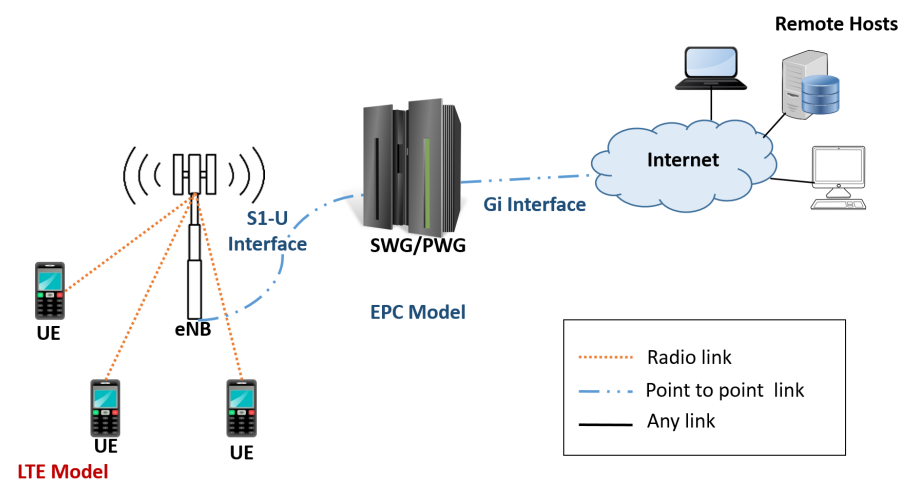

Figure 4.1: LTE model for NS-3 simulations, based on the LENA project [16]. All the important details of the LTE PHY and MAC protocols are implemented in the NS-3 simulations.

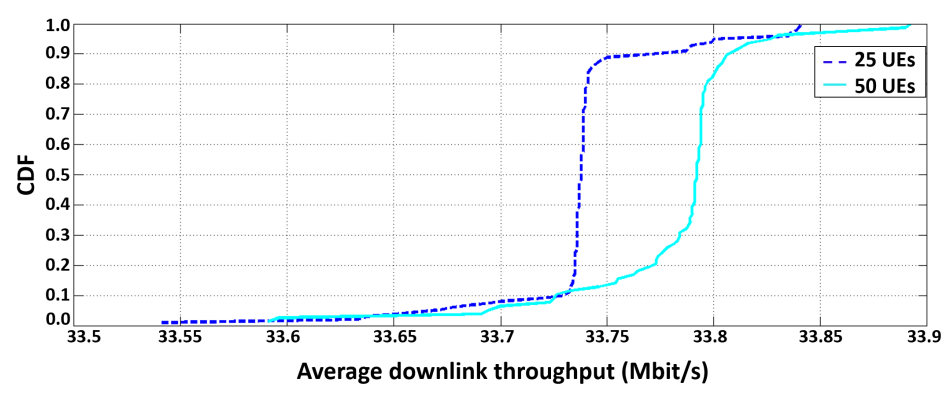

(a) Average downlink throughput (Mbits/s).

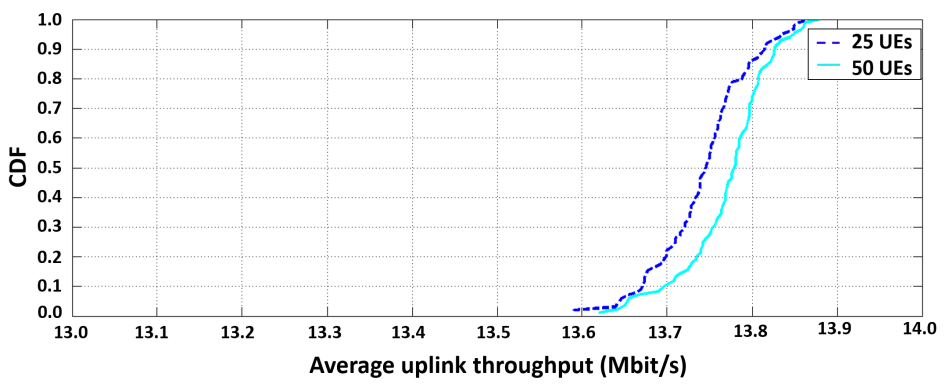

(b) Average uplink throughput (Mbits/s).

Figure 4.2: The CDF plot of simulated LTE NS-3 model; plot illustrates average uplink and downlink throughput (Mbits/s) with 25 and 50 UEs.

\subsubsection{Scenario:Average Downlink and Uplink Throughput}

In the constructed LTE simulation model, UEs were randomly distributed throughout the cell and follow a random walk mobility model in 2D. Fig. 4.2(a) and 
Fig. 4.2(b), show the CDF plot of the average downlink and uplink throughput seen across the 25, and 50 UEs. By intuition, it can be seen that in a relatively dense populated cell (with 25, and 50 UEs) the DL throughput is approximately $33 \mathrm{Mbit} / \mathrm{s}$ whereas the UL throughput is $13 \mathrm{Mbit} / \mathrm{s}$. A Monte Carlo experimental approach was used to obtain average throughput in each simulation iteration of 25 , and 50 UEs. These data rates can support applications such as IP-based multimedia services and also provide mission-critical real-time voice/video communications in PSC scenarios[265].

\subsubsection{Scenario: Aggregate throughput vs. Distance}

In this simulation scenario, we implement a macro-eNodeB site with a transmission power of $46 \mathrm{dBm}$. The simulation comprises $20 \mathrm{UEs}$ being randomly distributed throughout the cell, which has transmission power of $23 \mathrm{dBm}$, and follows random walk 2D mobility model. The round robin MAC scheduler is used to distribute the resources equally to all the users. The communication channel included in the simulation is trace fading loss model in a pedestrian scenario. The LTE band class 14 simulation uses a bandwidth of $10 \mathrm{MHz}$ and the frequency values is shown in Table 3.5.

Result observation: In this simulation scenario for data only transmission and reception, the aggregated DL throughput can be as large as $33 \mathrm{Mbit} / \mathrm{s}$ whereas the aggregated UL throughput can be as large as $11 \mathrm{Mbit} / \mathrm{s}$ as seen in Fig. 4.3. Further-

more, it is also observed that the throughput experience by the UEs is diminished as the distance the between the UE and macro-eNodeB increases. 


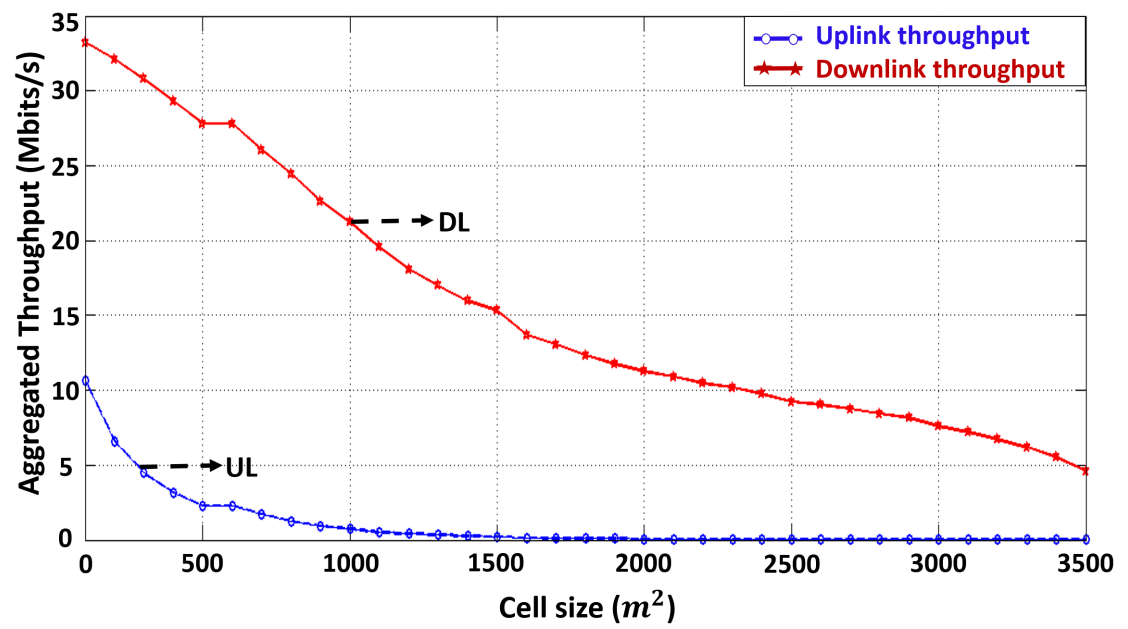

Figure 4.3: Aggregated uplink and downlink throughput observed by 20 UEs in the LTE band class 14. UEs experience better throughput when in close proximity with macro-eNodeB.

Table 4.1: LTE RF condition classification in [266].

\begin{tabular}{|c|c|c|c|}
\hline & RSRP $\mathbf{( d B m})$ & RSRQ $\mathbf{~ d B m ) ~}$ & SINR (dB) \\
\hline Excellent & $\geq-80$ & $\geq-10$ & $\geq 20$ \\
\hline Good & -80 to -90 & -10 to -15 & 13 to 20 \\
\hline Mid-cell & -90 to -100 & -15 to -20 & 0 to 13 \\
\hline Cell-edge & $\leq-100$ & $<-20$ & $\leq 0$ \\
\hline
\end{tabular}

\subsubsection{Scenario: Signal quality measurement}

In this simulation scenario, the UEs are placed at the cell center, post cell center, pre cell-edge and cell-edge regions of a macro-eNB site. The simulation model uses the simulation parameters and their attributes as shown in Table 3.5. The signal quality is a measurement of three quantities: namely RSRP (reference signal received power), RSRQ (reference signal received quality), and SINR. SINR is a measure of signal quality defined by a UE vendor but never reported to the network. The UEs report the CQI to the network indicating DL transmission rate. The CQI is a 4-bit integer and is based on the observed SINR at the UE. In this simulation scenario, SINR factor is used to measure the link quality and the chapter assumes the link quality is poor if the CQI value approaches 0. 


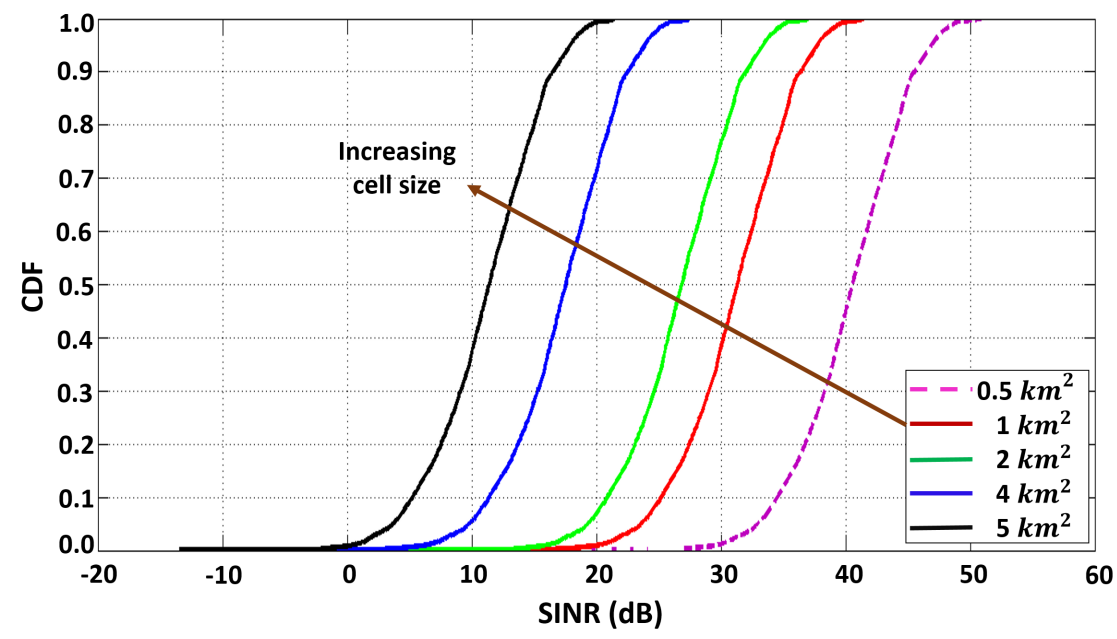

Figure 4.4: The CDF plot of DL SINR values measured by the UEs placed at cell center, post cell center, pre cell-edge, and cell-edge regions.

Result observation: The simulation cell size varies between $0.5 \mathrm{~km}^{2}$ to $5 \mathrm{~km}^{2}$. As the distance between the eNB and the UE gradually increases, a change in signal quality is observed. Fig. 4.4 shows the CDF plots of DL SINR values measured by the UEs throughout the cell, which range from $-10 \mathrm{~dB}$ to $50 \mathrm{~dB}$. These SINR values recorded by the UEs fit between the range of excellent to cell-edge RF conditions as specified in Table 4.1. The UEs when positioned nearby to the base station they experience better signal quality.

\subsubsection{Scenario: Inter-cell interference measurement}

The inter-cell interference in our simulation occurs in a homogeneous network with eNodeBs having the same capability, and same transmit power class. The inter-cell interference between two adjacent cell results in signal degradation and the UEs experience poor link quality at the cell-edge [114-116]. In the simulation model of the inter-cell interference measurement, 20 UEs are placed beyond the mid-cell region, and along the cell-edge region. The simulation model includes, two homogeneous 


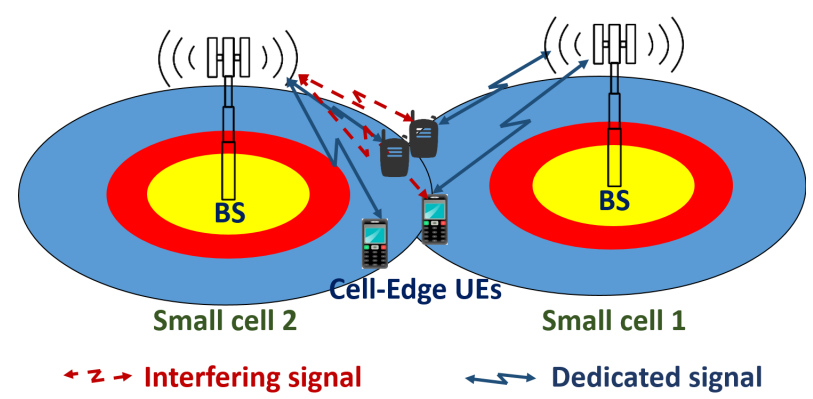

Figure 4.5: Inter-cell interference simulation scenario with cell-edge UEs with interference from neighboring cell. Such a scenario may e.g. correspond to two fire trucks or police cars that utilize LTE small cells, and are parked next to each other during an emergency incident.

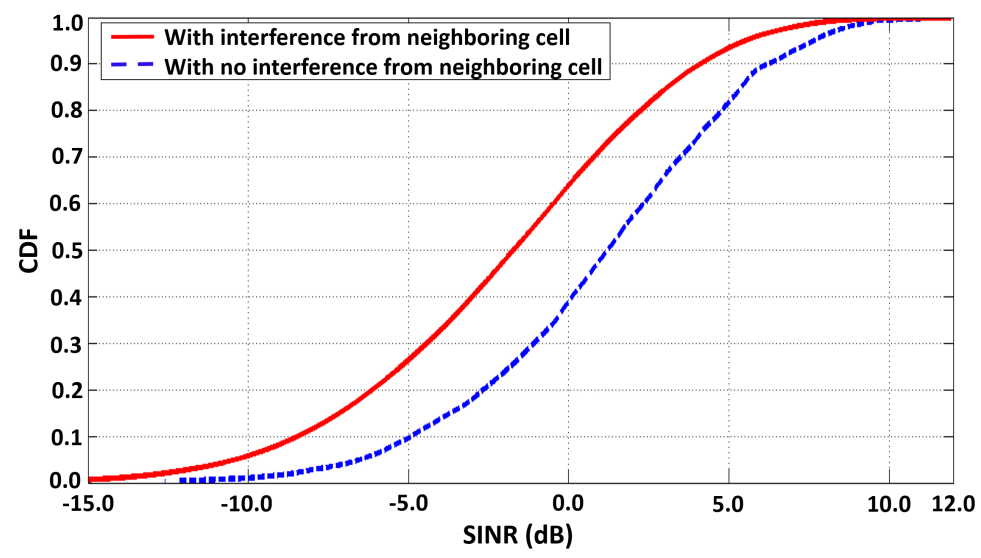

Figure 4.6: The CDF plot of DL SINR values measured by the UEs placed on the edge of a LTE cell 1 of Fig. 4.5.

eNodeBs with fixed location and transmission power of $23 \mathrm{dBm}, 20 \mathrm{UEs}$, round robin MAC scheduler, pedestrian fading model, MIMO 2-layer spatial multiplexing, a bandwidth of $10 \mathrm{MHz}$, and the other simulation parameters defined in Table 3.5. The simulation scenario is as shown in Fig. 4.5, which shows two adjacent homogeneous small cells and cell-edge UEs. The cell-edge UEs in cell 1 observe interference from the neighboring cell 2. The main goal of inter-cell interference simulation is to measure the signal degradation observed by UEs at cell-edge in cell 1 .

Result observation: The intuitive analysis of Fig. 4.6 conclude that the celledge UEs in Fig. 4.5 observe signal degradation and experience poor link quality 
when compared to cell-edge UEs with no interference. The SINR values measured by the cell-edged UEs in both the scenario are plotted in Fig. 4.6 in form of a CDF. The DL SINR values experienced by the UEs range from $-10 \mathrm{~dB}$ to $10 \mathrm{~dB}$ in the cell with no interference. On the other hand, the SINR values measured by the UEs in cell 1 fall in the range of $-15 \mathrm{~dB}$ to $10 \mathrm{~dB}$ due to the signal degradation caused by the adjacent cell.

\subsection{NS-3 Simulation Setup for APCO-25}

The main goal of APCO-25 simulation study is to compute the throughput, and the SINR distribution. Fig. 4.7 shows the APCO-25 simulation environment implemented in this chapter. The subscriber units in the simulation environment operate in direct mode, also known as talk-around, which enables EFR to have radio-to-radio direct communications by selecting a channel that bypasses their repeater/base systems. However this direct mode of communication limits the distance between mobile/portable subscriber units and spectrum reuse [93, 97]. A portable subscriber units can be described as a hand held device, while mobile subscriber units are mounted in EFR vehicle for mission-critical communication, to operates vehicle siren and lights. The APCO-25 Phase I and Phase II both can employ direct mode operations. Phase II TDMA systems require timing reference from the base station via system control channel and traffic channel for slot synchronization. The direct mode operation is challenging in case of Phase II TDMA due to the unavailability of master timing reference from the base station or a repeater [97]. In direct operation mode, as the distance between the subscriber units increases, the SINR value decreases. 


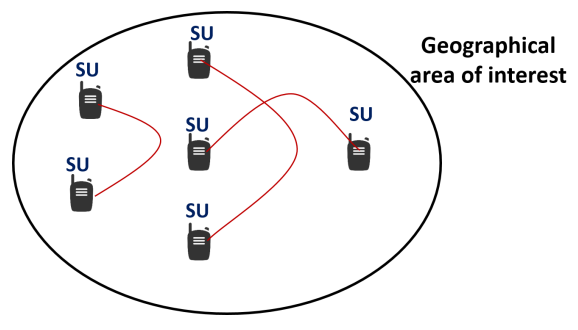

(a) The lines indicate direct communication mode between the subscriber units.

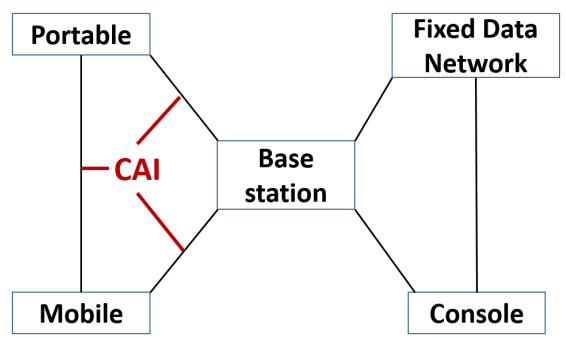

(b) APCO-25 CAI digital voice modulation standard in a radio system.

Figure 4.7: APCO-25 suite of standards for public safety communication referenced in NS-3 simulation.

The APCO-25 common air interface (CAI) is a specified standard for digital voice modulation used by the compliant radios as shown in Fig. 4.7b. Public safety devices form different vendors using APCO-25 CAI can communicate with each other. APCO-25 CAI has a control channel with a rate of 9600 bps and it uses improved multi-band excitation (IMBE) for voice digitization. The IMBE voice encoder-decoder (vocoder) samples the audio input into digital stream for transmission. At the receiver, vocoder produces synthetic equivalent audio from the digital stream [94, 97]. 


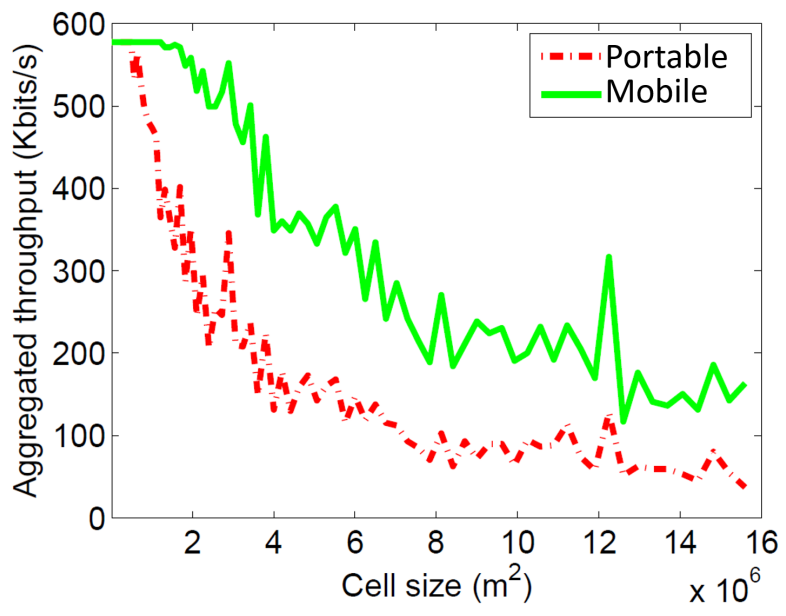

Figure 4.8: The maximum observed throughput in case of data communication is approximately $580 \mathrm{Kbits} / \mathrm{s}$ for both portable and mobile public safety devices.

\subsubsection{Scenario: Throughput and SINR Simulation of APCO- 25 Portable and Mobile Devices}

The NS-3 simulation for APCO-25, consist a square simulation area and with a uniform random distribution of 20 subscriber units across the cell. These 20 subscriber units in direct communication mode use APCO-25 CAI for digital voice modulation and Friis propagation model. The portable subscriber units have transmission power of 5 Watts, mobile subscriber units have transmission power of 10 Watts.

Result observation: In the direct communication mode, maximum throughput is possible when subscriber units are in proximity. As the geographical distance between the portable and mobile public safety devices increases, the throughput decreases as shown in Fig. 4.8. The simulation of APCO-25 for data only system and direct communication mode demonstrates an aggregate throughput of $580 \mathrm{Kbits} / \mathrm{s}$ as shown in Fig. 4.8. Whereas, Fig. 4.9 and Fig. 4.10 showcases the SINR observed by the portable and mobile public safety devices in the geographical area of interest. From Fig. 4.9 and Fig. 4.10 it is observed that the SINR values decreases with the 


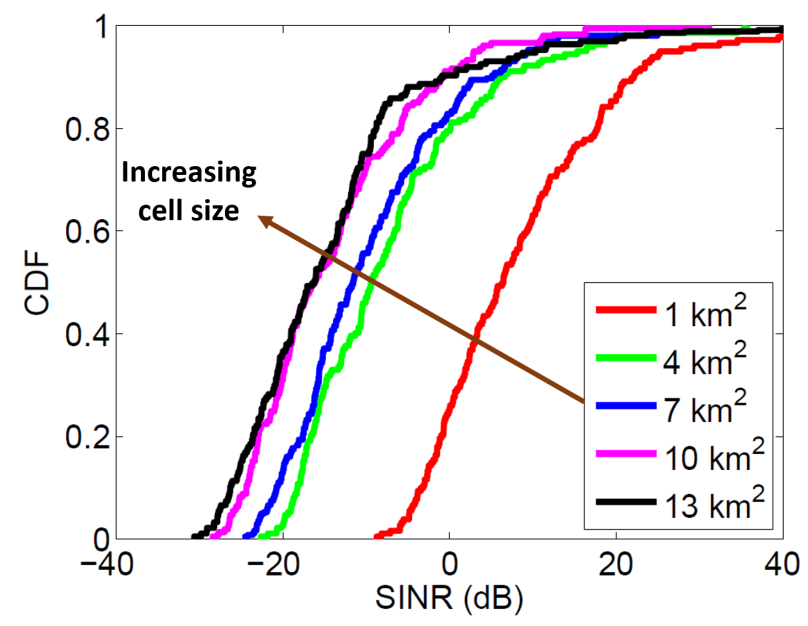

Figure 4.9: SINR CDF plots for APCO-25 portable subscriber units, with increasing distance between the subscriber units.

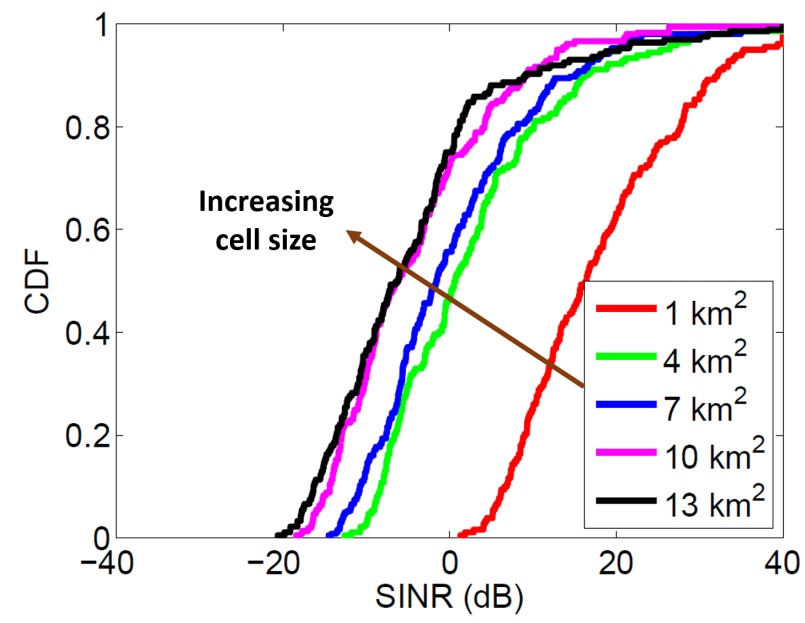

Figure 4.10: SINR CDF plots for APCO-25 mobile subscriber units, with increasing distance between the subscriber units.

increasing distance between two portable and mobile subscriber units. The observed SINR values range from $-30 \mathrm{~dB}$ to $40 \mathrm{~dB}$ for portable devices, whereas mobile devices observe SINR value between $-20 \mathrm{~dB}$ and $40 \mathrm{~dB}$.

Since the SINR experienced by the communicating subscriber units depends on the proximity between the subscriber units, it can be concluded that the SINR value decreases with increasing remoteness between the communicating subscriber units. Furthermore, it can also be also noticed, the increasing distance and poor link quality 
Table 4.2: Results summary of NS-3 simulation for LTE band class 14 and APCO25.

\begin{tabular}{|c|c|}
\hline Simulation scenario & Results observation \\
\hline $\begin{array}{l}\text { Band class 14: aggregate } \\
\text { throughput computation }\end{array}$ & $\begin{array}{l}\text { The main goal of this simulation scenario is to com- } \\
\text { pute aggregated data throughput for DL and UL. As } \\
\text { observed, the peak aggregated throughout for DL is } \\
33 \mathrm{Mbit} / \mathrm{s} \text { and for UL is } 11 \mathrm{Mbit} / \mathrm{s} \text { as seen in Fig. } 4.3 \text {. }\end{array}$ \\
\hline $\begin{array}{l}\text { Band class 14: signal quality } \\
\text { measurement }\end{array}$ & $\begin{array}{l}\text { The main goal of this simulation scenario is to measure } \\
\text { SINR experienced by UEs in a cell. As observed in } \\
\text { Fig. } 4.4 \text {, the signal quality degrades with increasing } \\
\text { distance between eNB and UE. The UEs experience } \\
\text { SINR value between }-10 d B \text { to } 50 d B \text {. }\end{array}$ \\
\hline $\begin{array}{l}\text { Band class 14: inter-cell inter- } \\
\text { ference measurement }\end{array}$ & $\begin{array}{l}\text { The main goal of this simulation is to calculate the } \\
\text { SINR experienced by the UE in cell-edge region with } \\
\text { and without interference from the adjacent cell. The } \\
\text { SINR experienced by UEs range from }-10 d B \text { to } 10 d B \\
\text { when no interference present. Whereas, in case of in- } \\
\text { terference from adjacent cell the UEs experience lower } \\
\text { link quality and range from }-15 d B \text { to } 10 d B \text { as seen } \\
\text { in Fig. } 4.5 \text {. }\end{array}$ \\
\hline $\begin{array}{l}\text { APCO-25: } \\
\text { throughput computation }\end{array}$ & $\begin{array}{l}\text { The main goal of this simulation scenario is to com- } \\
\text { pute aggregated data throughput for portable and mo- } \\
\text { bile subscriber units in direct communication mode. A } \\
\text { peak throughput of } 580 \mathrm{Kbits} / \mathrm{s} \text { is observed in case of } \\
\text { both portable and mobile subscriber units as seen in } \\
\text { Fig. } 4.8 \text {. }\end{array}$ \\
\hline $\begin{array}{lll}\text { APCO-25: } & \text { signal quality } \\
\text { measurement } & & \end{array}$ & $\begin{array}{l}\text { The main goal of this simulation scenario is to measure } \\
\text { SINR experienced by portable and mobile subscriber } \\
\text { units in a cell. As observed in Figs. } 4.9 \text { and } 4.10 \text {, } \\
\text { the signal quality degrades with increasing distance } \\
\text { between eNB and UE. The portable devices experience } \\
\text { SINR values ranging from }-30 \mathrm{~dB} \text { to } 40 \mathrm{~dB} \text {, whereas } \\
\text { mobile devices observe SINR value between }-20 \mathrm{~dB} \\
\text { to } 40 \mathrm{~dB} \text {. }\end{array}$ \\
\hline
\end{tabular}

lead to dropped packet which results in lower aggregated cell throughput. The mobile subscriber units have relatively higher transmission power compared to portable subscriber units. Therefore Fig. 4.8 also conclude that the mobile subscriber units demonstrate relatively higher throughput when compared to portable subscriber units. Furthermore, mobile subscriber units experience better SINR compared to portable subscriber units as shown in Fig. 4.9 and Fig. 4.10. 


\subsection{NS-3 Simulation Result Analysis}

The key factors for successful PSC can be broadly classified into mission-critical voice communication and wider coverage for public safety devices. From the comparison of Figs. 4.4, 4.9, and 4.10, it can be deduced that LMRS public safety devices experience larger cell coverage when compared to the band class 14 LTE devices. This volume of cell coverage is critical during the emergency scenario and is necessary to cover the maximum amount emergency prone area. Another rising factor in mission-critical PSC is real-time video communications, high data rate is needed to support real-time multimedia applications [265]. The aggregated throughput of LMRS infrastructure is less when compared to the aggregated throughput of band class 14 infrastructure and is shown in Fig. 4.3 and Fig. 4.8. These data rates experienced by the LTE UEs can definitely support the much needed real-time video communications in several of the PSC scenarios.

An adaptation of successful edition of mission-critical PTT over LTE would take some time [267]. In the meantime, applying the individual strength of LMRS and LTE into a converged public safety device to mission-critical voice and much needed mission-critical real-time data support can be beneficial to PSC. 


\section{CHAPTER 5}

\section{SOFTWARE-DEFINED RADIO EXPERIMENTATIONS IN APCO-25 AND PUBLIC SAFETY LTE FREQUENCY BANDS}

This chapter discusses the experiments conducted using SDR receiver such as the RTL-SDR RTL2832U [19] and HackRF [18]. SDR is an implementation of a radio communication system where the components that have normally implemented in hardware such as amplifiers, mixers, filters, modulators/demodulators, and detectors are instead implemented by means of software [125, 126, 268]. Most SDR receivers use a variable-frequency oscillator, mixer, and filter. The flexible SDR receiver helps in tuning to the desired frequency or a baseband [125, 269]. These flexible tuning characteristics can be used to tune into public safety signals.

SDR provides a low-cost infrastructure and more or less computer-driven environment which makes it an integral part of the public safety research, development, and test activities $[123,124]$. The continual development of SDR related hardware and software have assisted PSC researchers in gaining a better understanding of public safety signals. Furthermore, in this section we capture and analyze LMRS and public safety LTE signals and is shown via examples.

\subsection{Capturing APCO-25 signals}

The setup for the detection of APCO-25 signals consists HackRF connected to a personal computer equipped with a sound card and installed with SDRSharp freeware tool $[17,129]$. This setup is used as radio scanner to observe unencrypted APCOP25 digital radio voice spectrum using the instructions provided in [122]. Fig. 5.1 shows the observed Broward County public safety radio spectrum for frequency of 851.68 MHz with signal strength of $-36.59 \mathrm{~dB}$. The second embedded window is

a spectrogram which is a visual representation of the frequency spectrum of the 

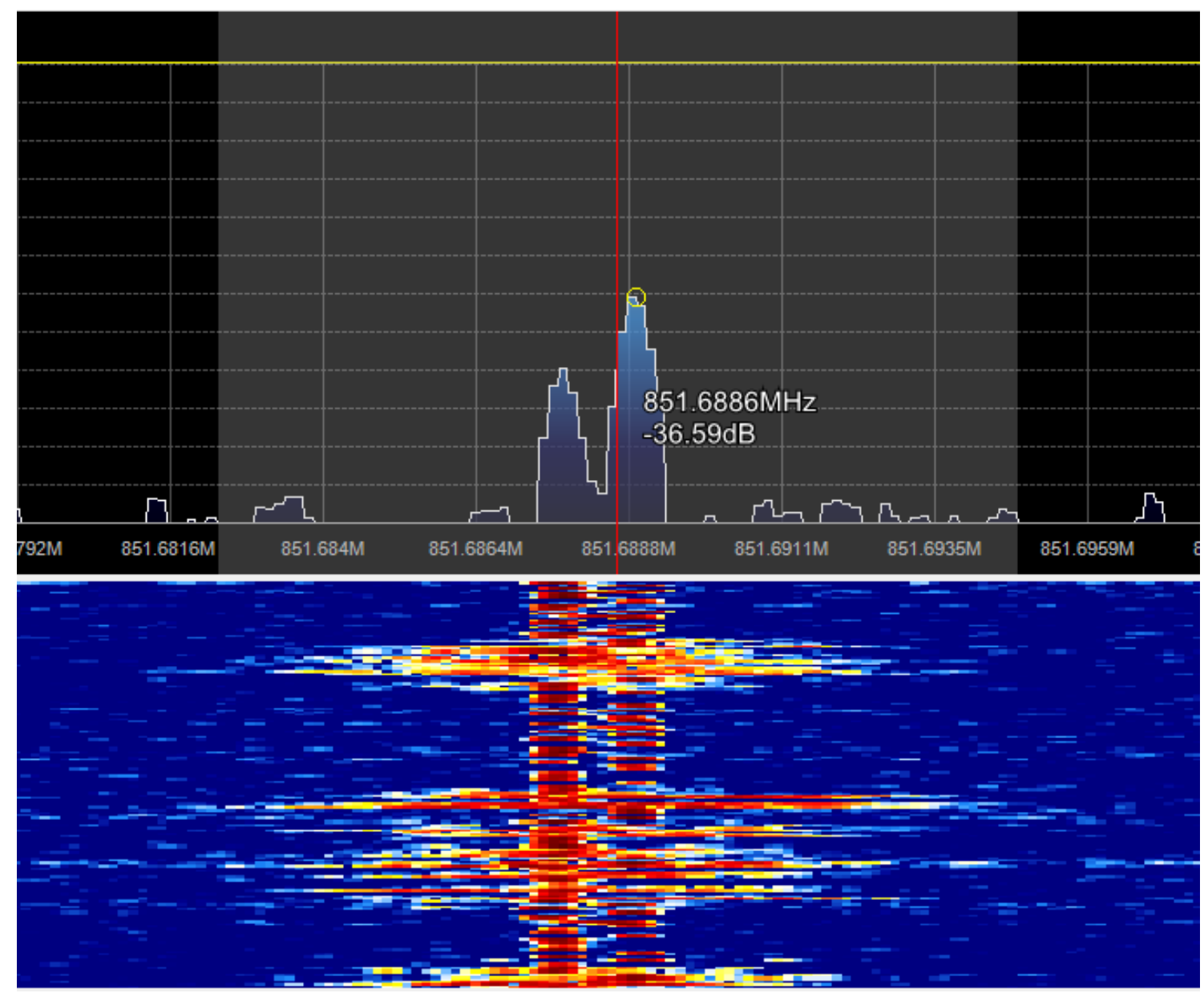

Figure 5.1: Broward County, FL, APCO-25 spectrum monitored using SDR\# software and captured using a HackRF receiver [17, 18].

received signal. HackRF has three receiver gain i.e., RF amplifier between $0 \mathrm{~dB}$ or $14 \mathrm{~dB}$, IF low noise amplifier $0 \mathrm{~dB}$ to $40 \mathrm{~dB}$, and baseband variable gain amplifier between $0 \mathrm{~dB}$ to $62 \mathrm{~dB}$. RF amplifier gain is set high to receive string signal, while keeping noise floor as low as possible. In this experiment the RF amplifier gain is set to $9 \mathrm{~dB}$. The two primary factors that describes window function are width of the main lobe and attenuation of the side lobes. 4-term Blackman-Harris window is better suited for spectral analysis [270] and therefore used in the current APCO-25 SDR experiments. 


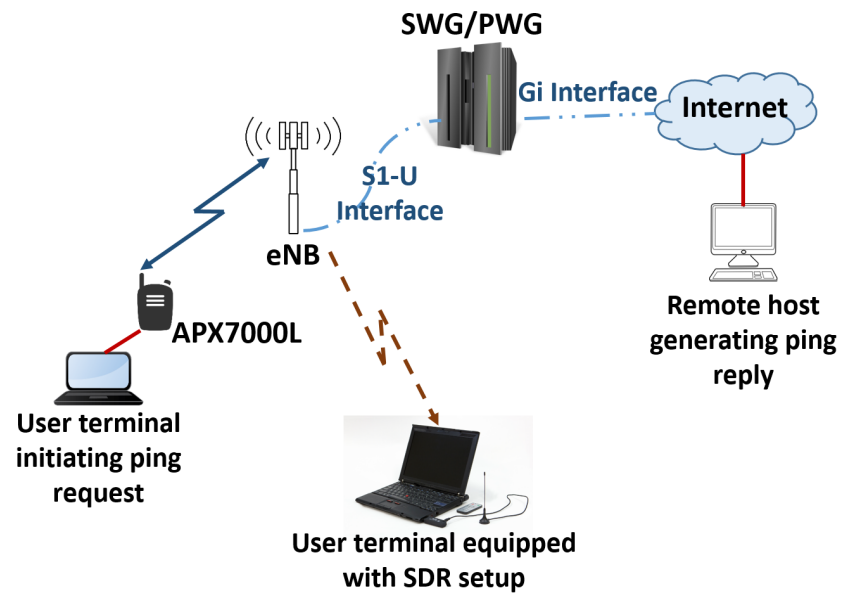

Figure 5.2: Setup for analyzing band class 14 spectrum.

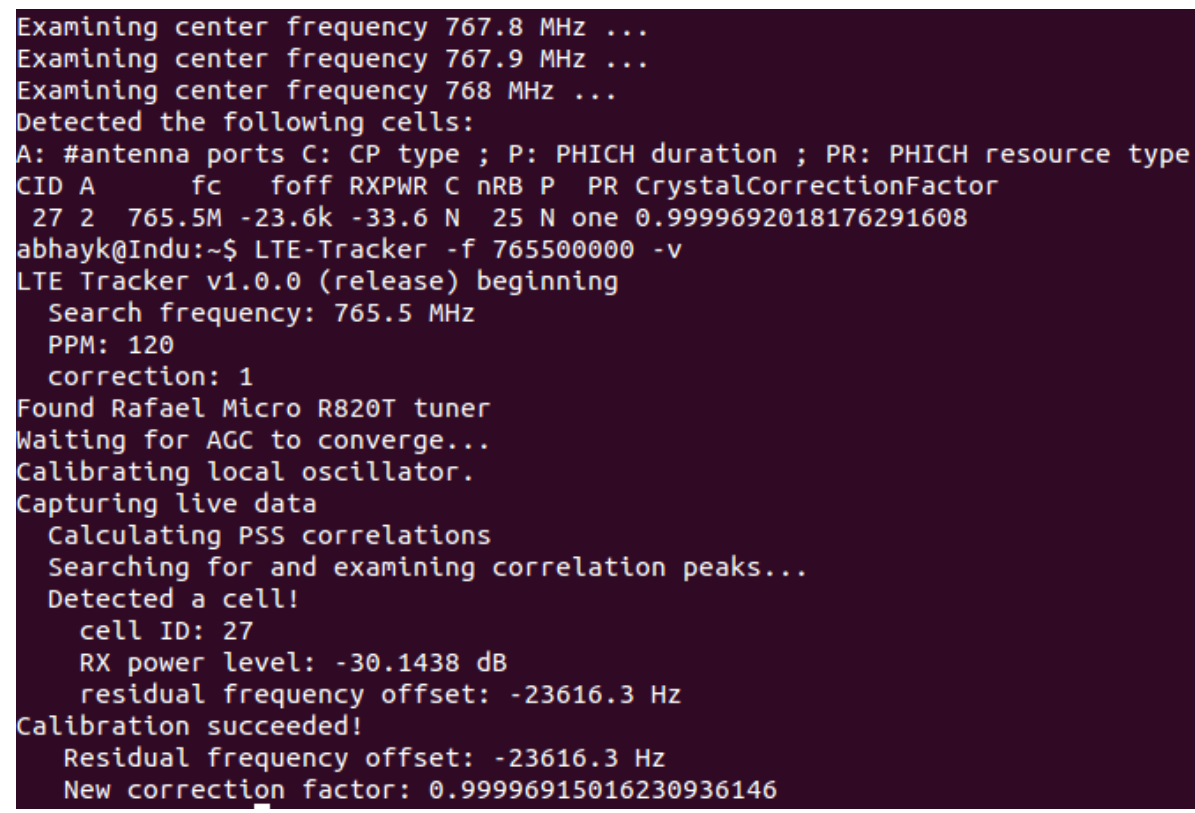

Figure 5.3: Public Safety Band class 14 cell detection using LTE-Tracker open source tool and RTL-SDR [19, 20].

\subsection{Capturing LTE Band Class 14}

The SDR experiment for analysis of band class 14 signals is conducted using the Motorola Solutions band class 14 infrastructure. The band class 14 base station in this scenario has DL frequency range of $758 \mathrm{MHz}$ to $768 \mathrm{MHz}$ whereas the UL fre- 


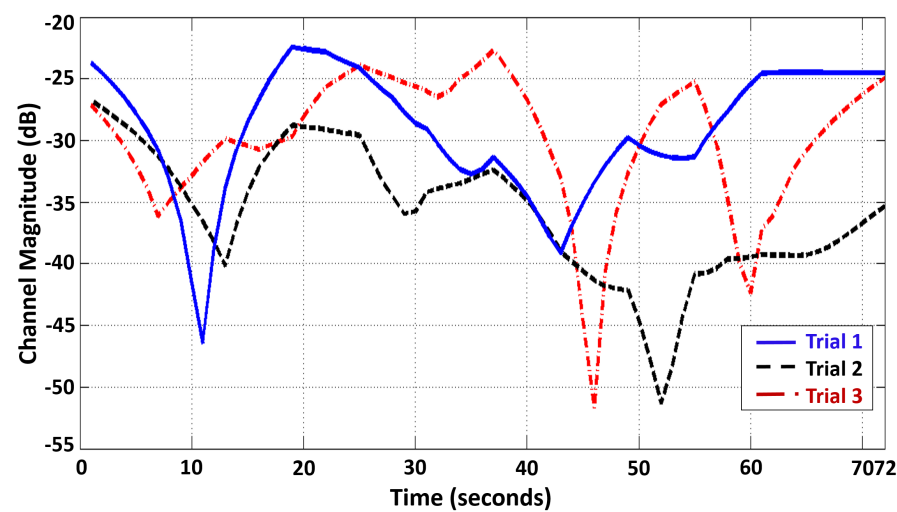

(a) Magnitude plot for the detected cell.

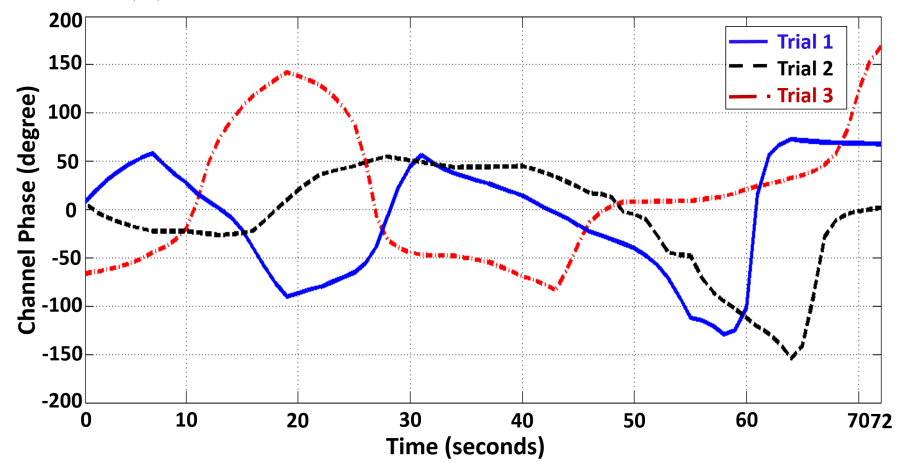

(b) Phase plot for the detected cell.

Figure 5.4: LTE cell tracker transfer function observed during various RTL-SDR experimentations. The variation in the channel magnitude and phase is observed due to the mobility of user terminal equipped with SDR setup.

quency range is $788 \mathrm{MHz}$ to $798 \mathrm{MHz}$ as specified in Fig. 3.5. Motorola APX7000L, an LTE-LMR converged device is treated as a UE in this scenario. Ping requests are initiated over a user terminal is routed via APX7000L to reach the remote host which generates the ping replies. An another user terminal equipped with SDR setup is used to capture these band class 14 signals as shown in Fig. 5.2. The user terminal equipped with SDR setup is running an open source LTE cell scanner and LTE tracker [20] tool to identify and track the band class 14 cell. Fig. 5.3 shows the identified band class 14 cell with cell ID 27, with two transmit antennas, center frequency of $765.5 \mathrm{MHz}$, and receive power level of $-33.6 \mathrm{~dB}$. Overall 25 resource blocks are occupied by the cell (channel bandwidth of $5 \mathrm{MHz}$ ), while the PHICH has 
normal duration and the resource type one. The transfer functions of the wireless channel from that port on the BS to the dongle's antenna are shown in Fig. 5.4. Fig. 5.4a shows the instantaneous magnitude transfer function of port 0 of cell 27 whereas Fig. 5.4b shows the phase plot.

These analyses of LTE band class 14 signals can assist PSC researchers in detecting coverage holes in PSN and gain knowledge on the quality of service experienced by the UEs. 


\section{CHAPTER 6}

\section{ANALYSIS OF FEICIC IN TWO-TIER AG-HETNET}

In this chapter, we investigate the performance of inter-cell interference coordination (ICIC) and cell range expansion (CRE) techniques for an LTE band class 14 PSC network as shown in Fig. 1.2 for a suburban environment. By randomly removing macro base stations (MBSs), we simulate a mock emergency situation to study the impact of interference and CRE when the UABSs are deployed. The two-tier AG-HetNet simulation model considers Rayleigh fading and simplified path loss model and Okumura-Hata model for calculating the propagation losses. Subsequently, we explore potential gains in 5pSE from the use of 3GPP Release 10/11 ICIC techniques. Furthermore, we jointly optimize the UABSs locations, CRE parameter of the UABSs, and ICIC parameters for both MBSs and UABSs.

\subsection{System Model}

In the two-tier AG-HetNet model, we consider a wireless network after a disaster as shown in Fig. 6.1. In particular, Fig. 6.1(a) shows that most of the geographical area in a typical PSC network is under SE coverage before a disaster. In the event of a disaster, the PSC network infrastructure is destroyed, and the first responders and victim users experience SE outage as illustrated by white areas in Fig. 6.1(b). In this scenario, the existing MBSs get overloaded with many UEs, and as a result, these UEs begin to experience poor QoS. Subsequently, at the site of emergency, the first responders and victim users located in the spectral efficiency (SE) outage regions will observe very low SE or possibly complete outage.

To address the SE problems in a scenario as in Fig. 6.1(b), We consider a two-tier HetNet deployment with MBSs and UABSs as shown in Fig. 1.2, where all the MBS and UABS locations (in three dimensions) are captured in matrices $\mathbf{X}_{\mathrm{mbs}} \in \mathbb{R}^{N_{\mathrm{mbs}} \times 3}$ 


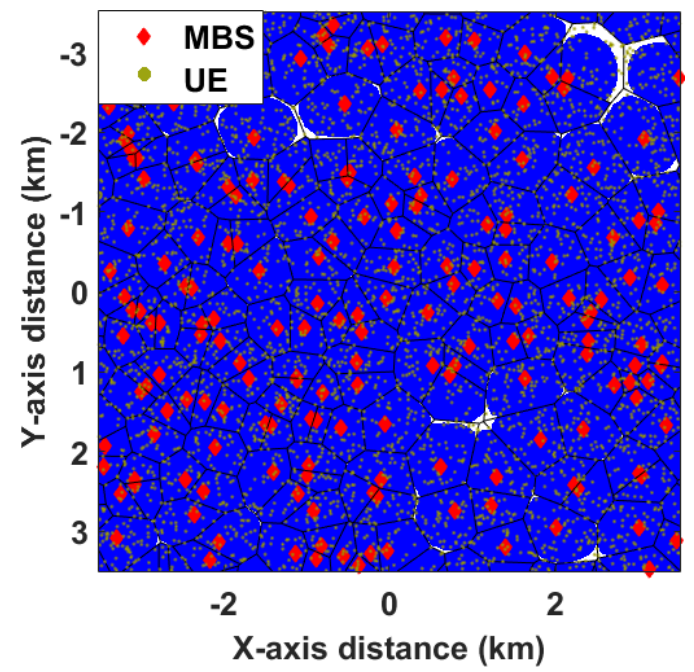

(a) Typical PSC network.

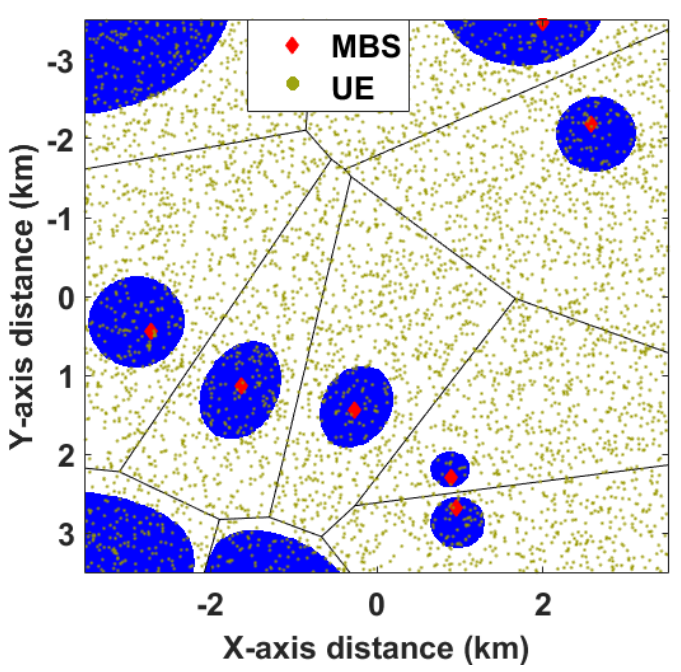

(b) PSC network after a disaster.

Figure 6.1: Wireless network coverage before/after a disaster with fixed terrestrial infrastructure.

and $\mathbf{X}_{\text {uabs }} \in \mathbb{R}^{N_{\text {uabs }} \times 3}$, respectively, where $N_{\text {mbs }}$ and $N_{\text {uabs }}$ denote the number of MBSs and UABSs within the simulation area. The MBS and user equipment (UE) locations are each modeled using a two-dimensional Poisson point process (PPP) with intensities ${ }^{\sim}$ mbs and ${ }^{u}$ ue, respectively [58, 271]. The UABSs are deployed at fixed height and their locations are either optimized using the genetic algorithm, or they are deployed on a fixed hexagonal grid. The heights for each of these wireless nodes are specified in Table 6.1.

We assume that the MBSs and the UABSs share a common transmission bandwidth, round robin scheduling is used in all downlink transmissions, and full buffer traffic is used in every cell. The transmit power of the MBS and UABS are $P_{\text {mbs }}$ and $P_{\text {uabs }}$, respectively, while $K$ and $K^{\prime}$ are the attenuation factors due to geometrical parameters of antennas for the MBS and the UABS, respectively. Then, the effective transmit power of the MBS is $P_{\mathrm{mbs}}^{\prime}=K P_{\mathrm{mbs}}$, while the effective transmit power of the UABS is $P_{\text {uabs }}^{\prime}=K^{\prime} P_{\text {uabs }}$. 
An arbitrary UE $n$ is always assumed to connect to the nearest MBS or UABS, where $n \in\left\{1,2, \ldots, N_{\text {ue }}\right\}$. Then, for the $n$th UE the reference symbol received power (RSRP) from the macro-cell of interest (MOI) and the UAV-cell of interest (UOI) are given by [58]

$$
S_{\mathrm{mbs}}\left(d_{m n}\right)=\frac{P_{\mathrm{mbs}}^{\prime} H}{10^{\varphi / 10}}, S_{\mathrm{uabs}}\left(d_{u n}\right)=\frac{P_{\mathrm{uabs}}^{\prime} H}{10^{\varphi^{\prime} / 10}}
$$

where the random variable $H \sim \operatorname{Exp}(1)$ accounts for Rayleigh fading, $\varphi$ is the pathloss observed from MBS in $\mathrm{dB}, \varphi^{\prime}$ is the path-loss observed from UABS in $\mathrm{dB}, d_{m n}$ is the distance from the nearest MOI, and $d_{u n}$ is the distance from the nearest UOI. In this chapter, the variation in deployment height of the UABSs and the effects of dominant line-of-sight links on 5pSE of the network are not treated explicitly and left as a future work. Hence, Rayleigh fading channel is considered instead of Rican fading channel in this system model.

\subsubsection{Path Loss Model}

Given the complexity of signal propagation, an accurate path-loss modeling would require complex ray tracing models and empirical measurements. To measure the path-loss observed by the $n$th UE, we use the simplified path loss model (SPLM), which is an approximation to the real propagation channel. However, for more accurate analysis of the signal reliability requirements in PSC networks, we also implement the free space suburban Okumura-Hata path loss model (OHPLM) with LTE band class 14 frequency [30, 272].

\section{Simplified Path Loss Model}

The SPLM gives a coarse analysis of signal propagation and is a function of path loss exponent and the distance between the serving base station and the $n$th UE [273]. 


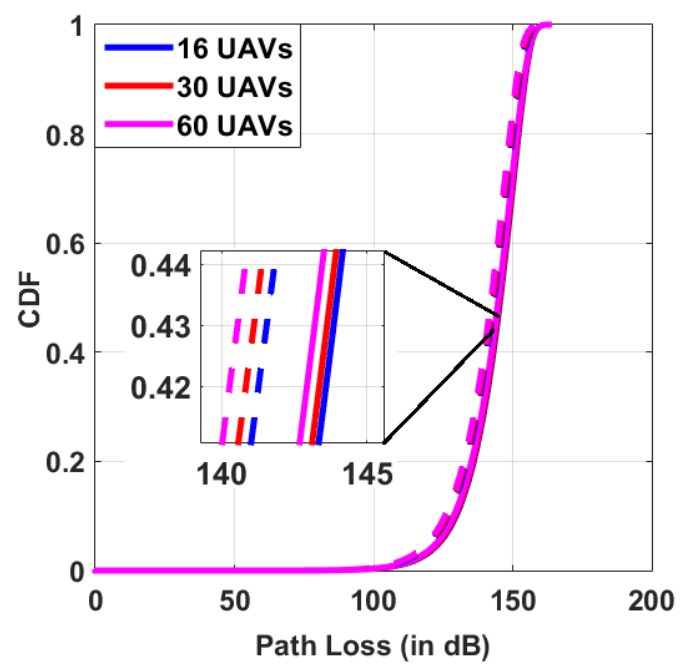

(a) CDF of simplified path loss model.

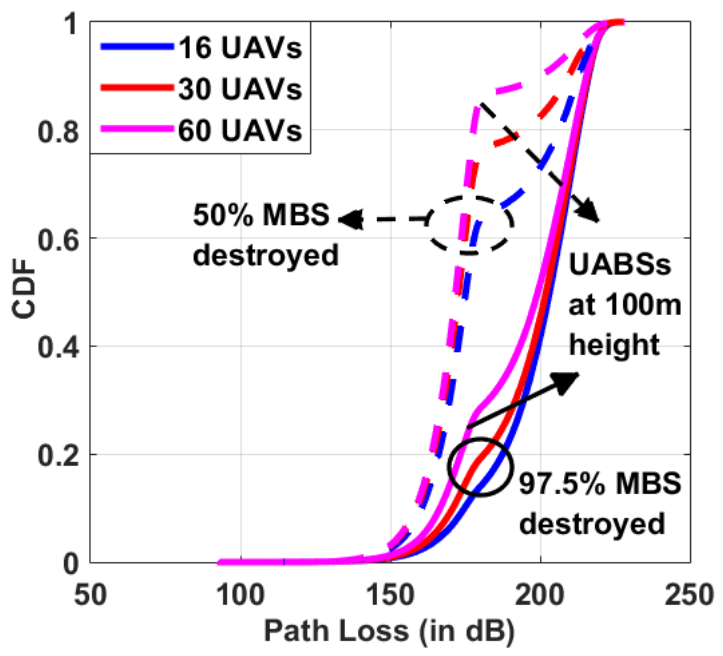

(b) CDF of Okumura-Hata model.

Figure 6.2: The CDF of the combined path loss observed from all the base stations in a two-tier AG-HetNet. Dashed lines correspond to the scenario with $50 \%$ of the MBS destroyed, while solid lines correspond to the scenario with $97.5 \%$ of the MBS destroyed.

The SPLM is a free space model and does not considered any physical structures or other obstacles which might affect the coverage of UABSs in real world deployments. Based on the SPLM, the path-loss (in $\mathrm{dB}$ ) observed by the $n$th UE from $m$ th MOI and $u$ th UOI is given by

$$
\varphi=10 \log _{10}\left(d_{m n}^{\delta}\right), \varphi^{\prime}=10 \log _{10}\left(d_{u n}^{\delta}\right)
$$

where $\delta$ is the path-loss exponent, and $d_{u n}$ depends on the locations of the UABSs that will be dynamically optimized.

Fig. 6.2(a) shows the empirical path-loss cumulative distribution functions (CDFs), calculated for all the distances between base stations $\left(\mathbf{X}_{\mathrm{mbs}}\right.$ and $\left.\mathbf{X}_{\mathrm{uabs}}\right)$ and UEs $\left(\mathbf{X}_{\mathrm{ue}}\right)$ using (6.2). For the path-loss exponent value defined in Table 6.1, we plot the CDFs for the cases when $50 \%$ and $97.5 \%$ of the MBS are destroyed. Inspection of Fig. 6.2(a) reveals the variation in CDFs is minimum for the different number of UABSs deployed and for a different number of the MBSs destroyed. This is because 
the SPLM does not consider external terrestrial factors. Nevertheless, the maximum allowable path-loss for the system is $160 \mathrm{~dB}$.

\section{Okumura-Hata Path Loss Model}

The OHPLM is more suited for a terrestrial environment with man-made structures and the environment in which the base-station height does not vary significantly $[274,275]$. This model is a function of the carrier frequency, distance between the UE and the serving cell, base station height, and UE antenna height $[274,276]$. Based on curve fitting of Okumura's original results, the path-loss (in dB) observed by the $n$th UE from MOI and UOI is given by $[277,278]$

$$
\begin{aligned}
& \varphi=A+B \log \left(d_{m n}\right)+C, \\
& \varphi^{\prime}=A+B \log \left(d_{u n}\right)+C,
\end{aligned}
$$

where the distances $d_{m n}$ and $d_{u n}$ are in $\mathrm{km}$, and the factors $A, B$, and $C$ depend on the carrier frequency and antenna height.

In a suburban environment, the factors $A, B, C$ are given by

$$
\begin{aligned}
& A=69.55+26.16 \log \left(f_{\mathrm{c}}\right)-13.82 \log \left(h_{\mathrm{bs}}\right)-a\left(h_{\mathrm{ue}}\right), \\
& B=44.9-6.55 \log \left(h_{\mathrm{bs}}\right) \\
& C=-2 \log \left(f_{\mathrm{c}} / 28\right)^{2}-5.4
\end{aligned}
$$

where $f_{\mathrm{c}}$ is the carrier frequency in $\mathrm{MHz}, h_{\mathrm{bs}}$ is the height of the base station in meter, and $a\left(h_{\mathrm{ue}}\right)$ is the correction factor for the UE antenna height $h_{\mathrm{ue}}$ in meter, which is defined as

$$
a\left(h_{\mathrm{ue}}\right)=1.1 \log \left(f_{\mathrm{c}}\right)-0.7 h_{\mathrm{ue}}-1.56 \log \left(f_{\mathrm{c}}\right)-0.8
$$

Furthermore, OHPLM assumes the carrier frequency $\left(f_{\mathrm{c}}\right)$ to be between 150 $\mathrm{MHz}$ to $1500 \mathrm{MHz}$, the height of the base station $\left(h_{\mathrm{bs}}\right)$ between $30 \mathrm{~m}$ to $200 \mathrm{~m}$, UE 
antenna height $\left(h_{\mathrm{ue}}\right)$ between $1 \mathrm{~m}$ to $10 \mathrm{~m}$, and the distances $d_{m n}$ and $d_{u n}$ between 1 $\mathrm{km}$ to $10 \mathrm{~km}[277,278]$. Nevertheless, the simulation values considered for OHPLM are defined in Table 6.1.

In Fig. 6.2(b), we plot the empirical path-loss CDFs using (6.3)-(6.8) and the OHPLM parameters in Table 6.1. Moreover, we plot the OHPLM path-loss CDFs for the cases when $50 \%$ and $97.5 \%$ of the MBS are destroyed. Inspection of Fig. 6.2(b) reveals a step-wise distribution of path-loss in the CDFs. This behavior is due to the variation in the height of base-stations, i.e., UABSs are deployed at the height of $100 \mathrm{~m}$ (larger path loss) while the height of MBSs is $30 \mathrm{~m}$ (smaller path loss). With $50 \%$ of the MBS destroyed, it can be seen that most UEs are connected to the MBSs, while with $97.5 \%$ of the MBS destroyed most UEs are served by the UABSs. Regardless, the maximum allowable path-loss when $50 \%$ and $97.5 \%$ of the MBSs are destroyed is $225 \mathrm{~dB}$ as shown in Fig. 6.2(b).

\subsubsection{GPP Release 10/11 Inter-Cell Interference Coordi- nation}

Due to their low transmission power, the UABSs are unable to associate a larger number of UEs compared to that of MBSs. However, by using the cell range expansion (CRE) technique defined in 3GPP Release-8, UABSs can associate a large number of UEs by offloading traffic from the MBSs. A negative side effect of CRE includes increased interference in the downlink of cell-edge UEs or the UEs in CRE region of the UABS, which is addressed by using ICIC techniques in LTE and LTEAdvanced [279-281].

3GPP Release-10 introduced a time-domain based enhanced ICIC (eICIC) technique to address interference problems. In particular, it uses ABSs which require the 


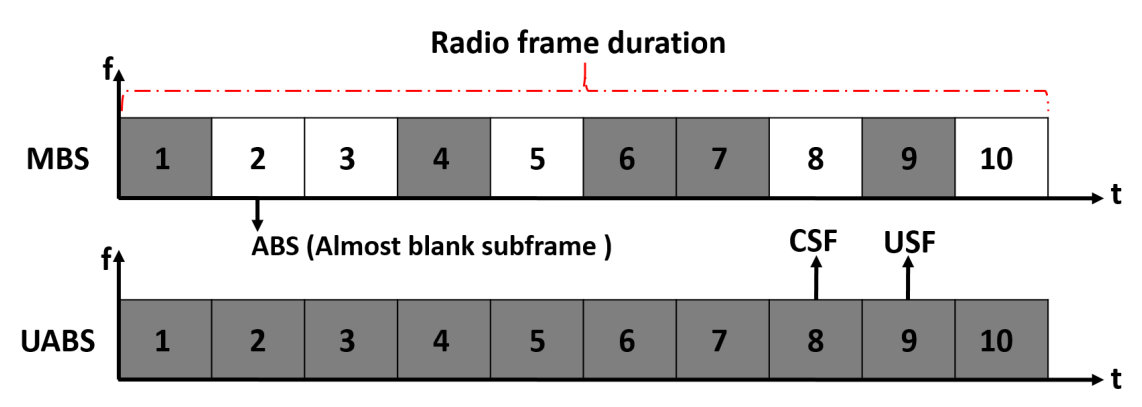

(a) 3GPP Release-10 eICIC with ABS.

Radio frame duration

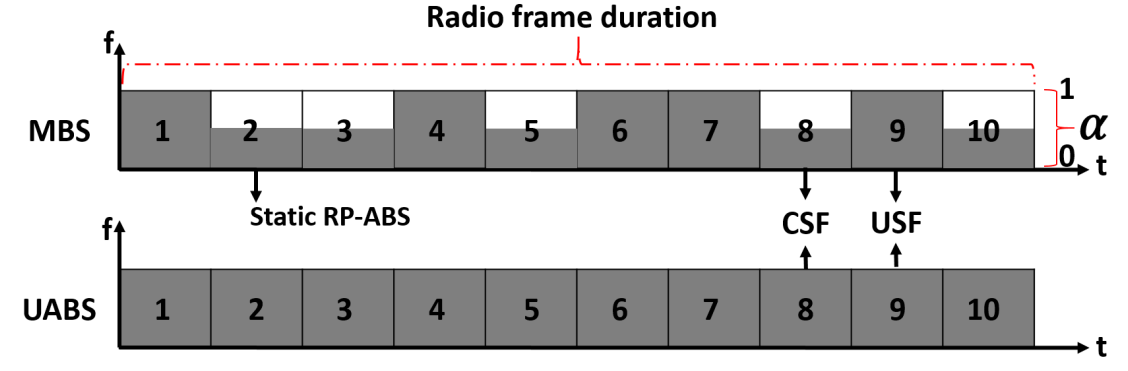

(b) 3GPP Release-11 FeICIC with reduced power ABS (RP-ABS).

Figure 6.3: LTE-Advanced frame structures for time-domain ICIC in two-tier AGHetNet. Certain UABS subframes are protected from MBS.

MBS to completely blank the transmit power on the physical downlink shared channel (PDSCH) resource elements as shown in Fig. 6.3(a). This separates the radio frames into coordinated subframes (CSF) and uncoordinated subframes (USF). On the other hand, 3GPP Release-11 defines further-enhanced ICIC (FeICIC), where the data on PDSCH is still transmitted but at a reduced power level as shown in Fig. 6.3(b).

The MBSs can schedule their UEs either in USF or in CSF based on the scheduling threshold $\rho$. Similarly, the UABSs can schedule their UEs either in USF or in CSF based on the scheduling threshold $\rho^{\prime}$. Let $\beta$ denote the USF duty cycle, defined as the ratio of number of USF subframes to the total number of subframes in a radio frame. Then, the duty cycle of CSFs is $(1-\beta)$. For ease of simulation, in this chapter, we consider that the USF duty cycle $\beta$ is fixed at 0.5 for all the MBSs, which is shown in [58] to have limited effect on system performance when $\rho$ and $\rho^{\prime}$ are 
optimized. Finally, let $0 \leq \alpha \leq 1$ denote the power reduction factor in coordinated subframes of the MBS for the FeICIC technique; $\alpha=0$ corresponds to Release-10 eICIC, while $\alpha=1$ corresponds to no ICIC (e.g., as in LTE Release-8). We assume that the ABS and reduced power pattern are shared via the X2 interface, which is a logical interface between the base stations.

Given the eICIC and FeICIC framework in 3GPP LTE-Advanced as in Fig. 6.3, and following an approach similar to that in [58] for a HetNet scenario, the signalto-interference ratio (SIR) experienced by an arbitrary UE can be defined for CSFs and USFs for the MOI and the UOI as follows:

$$
\begin{aligned}
\Gamma & =\frac{S_{\mathrm{mbs}}\left(d_{m n}\right)}{S_{\mathrm{uabs}}\left(d_{u n}\right)+Z}, \rightarrow \text { USF SIR from MOI } \\
\Gamma_{\mathrm{csf}} & =\frac{\alpha S_{\mathrm{mbs}}\left(d_{m n}\right)}{S_{\mathrm{uabs}}\left(d_{u n}\right)+Z} \rightarrow \text { CSF SIR from MOI, } \\
\Gamma^{\prime} & =\frac{S_{\mathrm{uabs}}\left(d_{u n}\right)}{S_{\mathrm{mbs}}\left(d_{m n}\right)+Z} \rightarrow \mathrm{USF} \text { SIR from UOI, } \\
\Gamma_{\mathrm{csf}}^{\prime} & =\frac{S_{\mathrm{uabs}}\left(d_{u n}\right)}{\alpha S_{\mathrm{mbs}}\left(d_{m n}\right)+Z} \rightarrow \mathrm{CSF} \text { SIR from UOI, }
\end{aligned}
$$

where $Z$ is the total interference power at a UE during USF or CSF from all the MBSs and UABSs, excluding the MOI and the UOI. In hexagonal grid UABS deployment model (and in [58]), locations of the UABSs (and small cells) are fixed. To maximize the 5pSE of the network, in this chapter we actively consider the SIRs in (6.9)-(6.12) while optimizing the locations of the UABSs using the genetic algorithm.

\subsubsection{UE Association and Scheduling}

The cell selection process relies on $\Gamma$ and $\Gamma^{\prime}$ in (6.9) and (6.11), respectively, for the MOI and UOI SIRs, as well as the CRE $\tau$. If $\tau \Gamma^{\prime}$ is less than $\Gamma$, then the 
UE is associated with the MOI; otherwise, it is associated with the UOI. After cell selection, the MBS-UE (MUE) and UABS-UE (UUE) can be scheduled either in USF or in CSF radio subframes as:

$$
\begin{aligned}
& \text { If } \Gamma>\tau \Gamma^{\prime} \text { and } \Gamma \leq \rho \rightarrow \mathrm{USF}-\mathrm{MUE}, \\
& \text { If } \Gamma>\tau \Gamma^{\prime} \text { and } \Gamma>\rho \rightarrow \mathrm{CSF}-\mathrm{MUE}, \\
& \text { If } \Gamma \leq \tau \Gamma^{\prime} \text { and } \Gamma^{\prime}>\rho^{\prime} \rightarrow \mathrm{USF}-\mathrm{UUE}, \\
& \text { If } \Gamma \leq \tau \Gamma^{\prime} \text { and } \Gamma^{\prime} \leq \rho^{\prime} \rightarrow \mathrm{CSF}-\mathrm{UUE} \text {. }
\end{aligned}
$$

Once a UE is assigned to a MOI/UOI and is scheduled within the USF/CSF radio frames, then the SE for this UE can be expressed for the four different scenarios in (6.13)-(6.16) as follows:

$$
\begin{aligned}
& C_{\mathrm{usf}}^{\mathrm{mbs}}=\frac{\beta \log _{2}(1+\Gamma)}{N_{\mathrm{usf}}^{\mathrm{mbs}}}, \\
& C_{\mathrm{csf}}^{\mathrm{mbs}}=\frac{(1-\beta) \log _{2}\left(1+\Gamma_{\mathrm{csf}}\right)}{N_{\mathrm{csf}}^{\mathrm{mbs}}}, \\
& C_{\mathrm{usf}}^{\mathrm{uabs}}=\frac{\beta \log _{2}\left(1+\Gamma^{\prime}\right)}{N_{\mathrm{usf}}^{\mathrm{uabs}}}, \\
& C_{\mathrm{csf}}^{\mathrm{uabs}}=\frac{(1-\beta) \log _{2}\left(1+\Gamma_{\mathrm{csf}}^{\prime}\right)}{N_{\mathrm{csf}}^{\mathrm{uabs}}},
\end{aligned}
$$

where $N_{\mathrm{usf}}^{\mathrm{mbs}}, N_{\mathrm{csf}}^{\mathrm{mbs}}, N_{\mathrm{usf}}^{\mathrm{uabs}}$, and $N_{\mathrm{csf}}^{\mathrm{uabs}}$ are the number of MUEs and UUEs scheduled in USF and CSF radio subframes, and $\Gamma, \Gamma_{\mathrm{csf}}, \Gamma^{\prime}, \Gamma_{\mathrm{csf}}^{\prime}$ are as in (6.9)-(6.12).

In this chapter, we consider only $5 \mathrm{pSE}$ is considered as the KPI for the two-tier AG-HetNet. The use of 5pSE which corresponds to the worst fifth percentile UE capacity among the capacities of all the $N_{\text {ue }}$ UEs (calculated based on (6.17)-(6.20)) within the simulation area. We believe it is a critical metric particularly for PSC scenarios to maintain a minimum QoS level at all the UEs in the environment. We 
define the dependency of the 5pSE to UABS locations and ICIC parameters as

$$
C_{5 \text { th }}\left(\mathbf{X}_{\text {uabs }}, \mathbf{S}_{\mathrm{mbs}}^{\mathrm{ICIC}}, \mathbf{S}_{\mathrm{uabs}}^{\mathrm{ICIC}}\right)
$$

where $\mathbf{X}_{\text {uabs }} \in \mathbb{R}^{N_{\text {uabs }} \times 3}$ captures the UABS locations as defined earlier, $\mathbf{S}_{\text {mbs }}^{\text {ICIC }}=$ $[\boldsymbol{\alpha}, \boldsymbol{\rho}] \in \mathbb{R}^{N_{\mathrm{mbs}} \times 2}$ is a matrix that captures individual ICIC parameters for each MBS, while $\mathbf{S}_{\text {uabs }}^{\text {ICIC }}=\left[\boldsymbol{\tau}, \boldsymbol{\rho}^{\prime}\right] \in \mathbb{R}^{N_{\text {uabs }} \times 2}$ is a matrix that captures individual ICIC parameters for each UABS. In particular,

$$
\boldsymbol{\alpha}=\left[\alpha_{1}, \ldots, \alpha_{N_{\mathrm{mbs}}}\right]^{T}, \quad \boldsymbol{\rho}=\left[\rho_{1}, \ldots, \rho_{N_{\mathrm{mbs}}}\right]^{T}
$$

are $N_{\mathrm{mbs}} \times 1$ vectors that include the power reduction factor and MUE scheduling threshold parameters for each MBS. On the other hand,

$$
\boldsymbol{\tau}=\left[\tau_{1}, \ldots, \tau_{N_{\text {uabs }}}\right]^{T}, \quad \boldsymbol{\rho}^{\prime}=\left[\rho_{1}^{\prime}, \ldots, \rho_{N_{\text {uabs }}}^{\prime}\right]^{T}
$$

are $N_{\text {uabs }} \times 1$ vectors that involve the CRE bias and UUE scheduling threshold at each UABS.

As noted in Section 6.1.2, the duty cycle $\beta$ of ABS and reduced power subframes is assumed to be set to 0.5 at all MBSs to reduce search space and complexity.

Considering that the optimum values of the vectors $\boldsymbol{\alpha}, \boldsymbol{\rho}, \boldsymbol{\rho}^{\prime}$, and $\boldsymbol{\tau}$ are to be searched over a multi-dimensional space, computational complexity of finding the optimum parameters is prohibitively high. Hence, to reduce the system complexity (and simulation runtime) significantly, we consider the same ICIC parameters are used for all MBSs and for all UABSs. In particular, we consider that for $i=$ $1, \ldots, N_{\text {mbs }}$ we have $\alpha_{i}=\alpha$ and $\rho_{i}=\rho$, while for $j=1, \ldots, N_{\text {uabs }}$ we have $\tau_{j}=\tau$ and $\rho_{j}^{\prime}=\rho^{\prime}$. Therefore, the dependence of the $5 \mathrm{pSE}$ on the UABS locations and ICIC parameters can be simplified as

$$
C_{5 \operatorname{th}}\left(\mathbf{X}_{\mathrm{uabs}}, \alpha, \rho, \tau, \rho^{\prime}\right)
$$




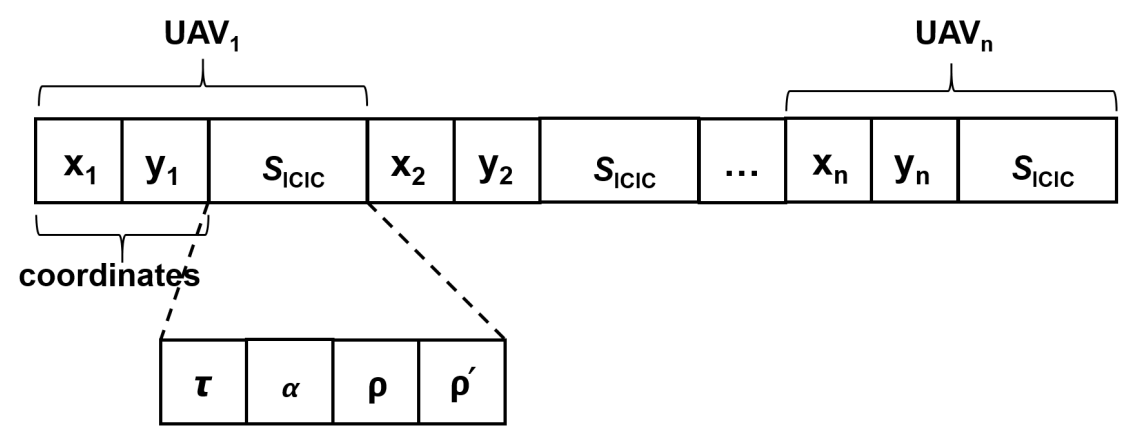

Figure 6.4: An example of a chromosome for FeICIC simulation in two-tier AGHetNet, where the UABS locations, ICIC parameter $\tau, \alpha, \rho$, and $\rho^{\prime}$ are optimized. The ICIC parameter $\beta$ is not optimized and is fixed at $50 \%$ duty cycle.

which we will seek ways to maximize in the next section.

The problem of individually optimizing ICIC parameters for the MBSs and UABSs is not considered as part of the dissertation due to the high computational complexity of the problem. The optimization of ICIC parameters for individual base-stations has been addressed in one of co-authored conference proceedings [52].

\subsection{UABS Deployment Optimization}

In this section, we discuss the UABS deployment using the genetic algorithm (GA) and the hexagonal grid model, where we use the 5pSE as an optimization metric to maximize for both scenarios.

\subsubsection{Genetic Algorithm based UABS Deployment Opti- mization}

The GA is a population-based optimization technique that can search a large environment simultaneously to reach an optimal solution [35]. In this chapter, the UABS coordinates and the ICIC parameters constitute the GA population, and a 


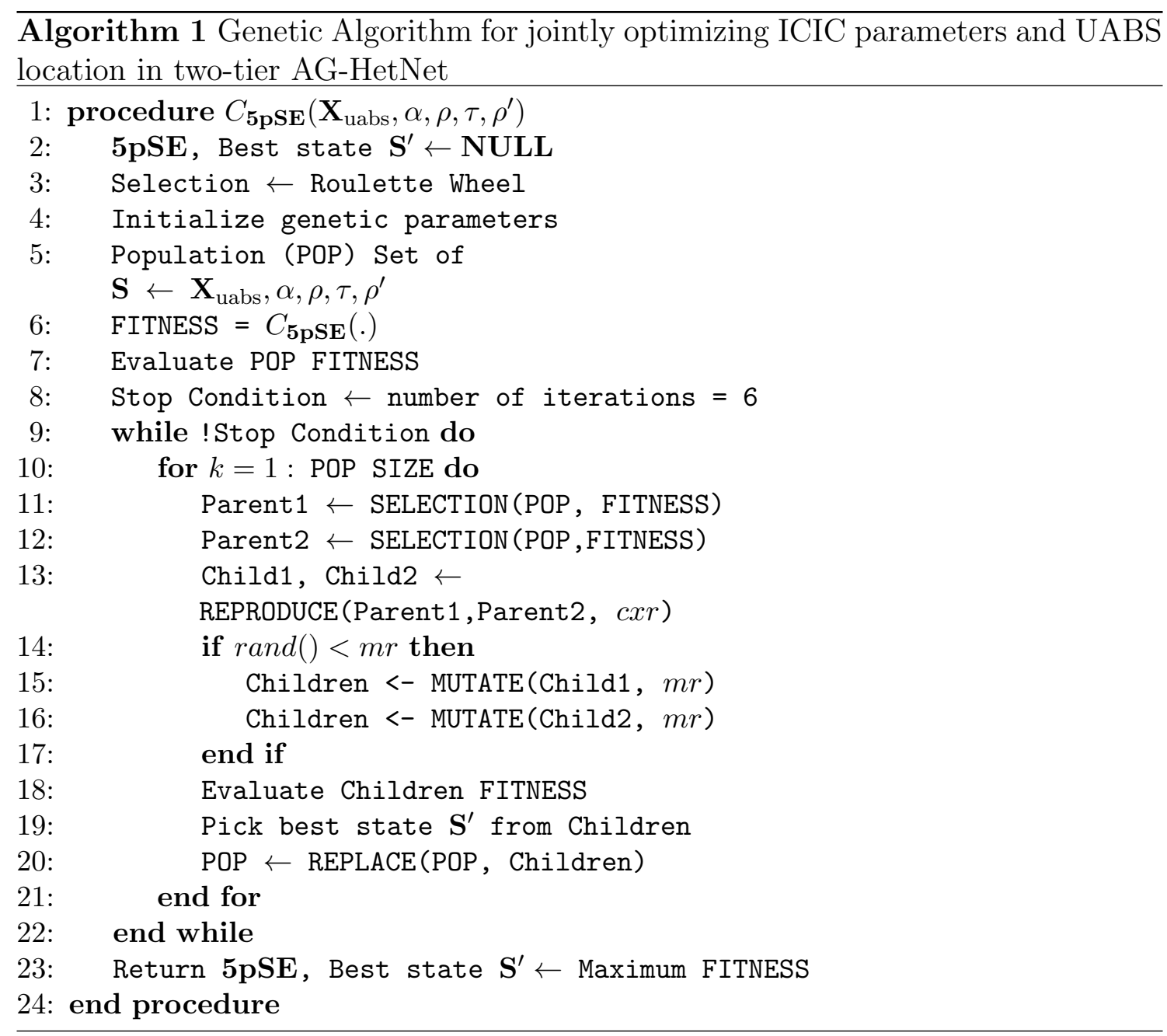

subsequent chromosome is illustrated in Fig. 6.4. Applying the detailed steps described in [35], Algorithm 1 describes the main steps used to optimize the UABS locations and ICIC parameters while computing the 5pSE.

We apply the GA to simultaneously optimize the UABS locations and ICIC network parameters in order to maximize the 5pSE of the network over a given geographical area of interest. The location of each UABS within a rectangular simulation area is given by $\left(x_{i}, y_{i}\right)$ where $i \in\left\{1,2, \ldots, N_{\text {uabs }}\right\}$. The UABS locations and the ICIC parameters that maximize the 5pSE objective function can be calculated 


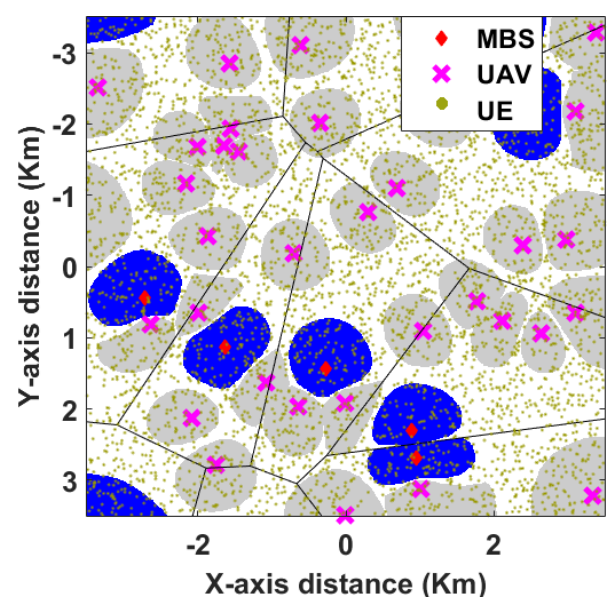

Figure 6.5: Two-tier PSC AG-HetNet after a disaster with UABS locations optimized using the GA (UAV height: $100 \mathrm{~m}$ ).

as

$$
\left[\hat{\mathbf{X}}_{\text {uabs }}, \hat{\alpha}, \hat{\rho}, \hat{\tau}, \hat{\rho^{\prime}}\right]=\arg \underset{X_{\text {uabs }}, \alpha, \rho, \tau, \rho^{\prime}}{\max } C_{5 \mathrm{th}}\left(\mathbf{X}_{\mathrm{uabs}}, \alpha, \rho, \tau, \rho^{\prime}\right)
$$

Since searching for optimal $\mathbf{X}_{\text {uabs }}$ and ICIC parameters through a brute force approach is computationally intensive, in this chapter we use the GA to find optimum UABS locations and the best-fit ICIC parameters $\tau, \alpha, \rho$, and $\rho^{\prime}$.

For the MBS locations shown in Fig. 6.1(b), an example outcome of UABS locations using the GA is shown in Fig. 6.5. Given the mobility and agility of UABSs, using the GA, the UAV positions can be dynamically rearranged to optimized locations to achieve the best network performance at the site of an emergency.

\subsubsection{UABS Deployment in a Hexagonal Grid}

As a lower complexity alternative to optimizing UABS locations, we consider deploying the UABSs on a hexagonal grid, where the positions of UABSs are deterministic. We assume that the UABSs are placed within the rectangular simulation area regardless of the existing MBS locations. The $5 \mathrm{pSE}$ for this network is determined 
Algorithm 2 Brute force algorithm jointly optimizing ICIC parameters in two-tier AG-HetNet and UABS deployed on fixed hexagonal grid.

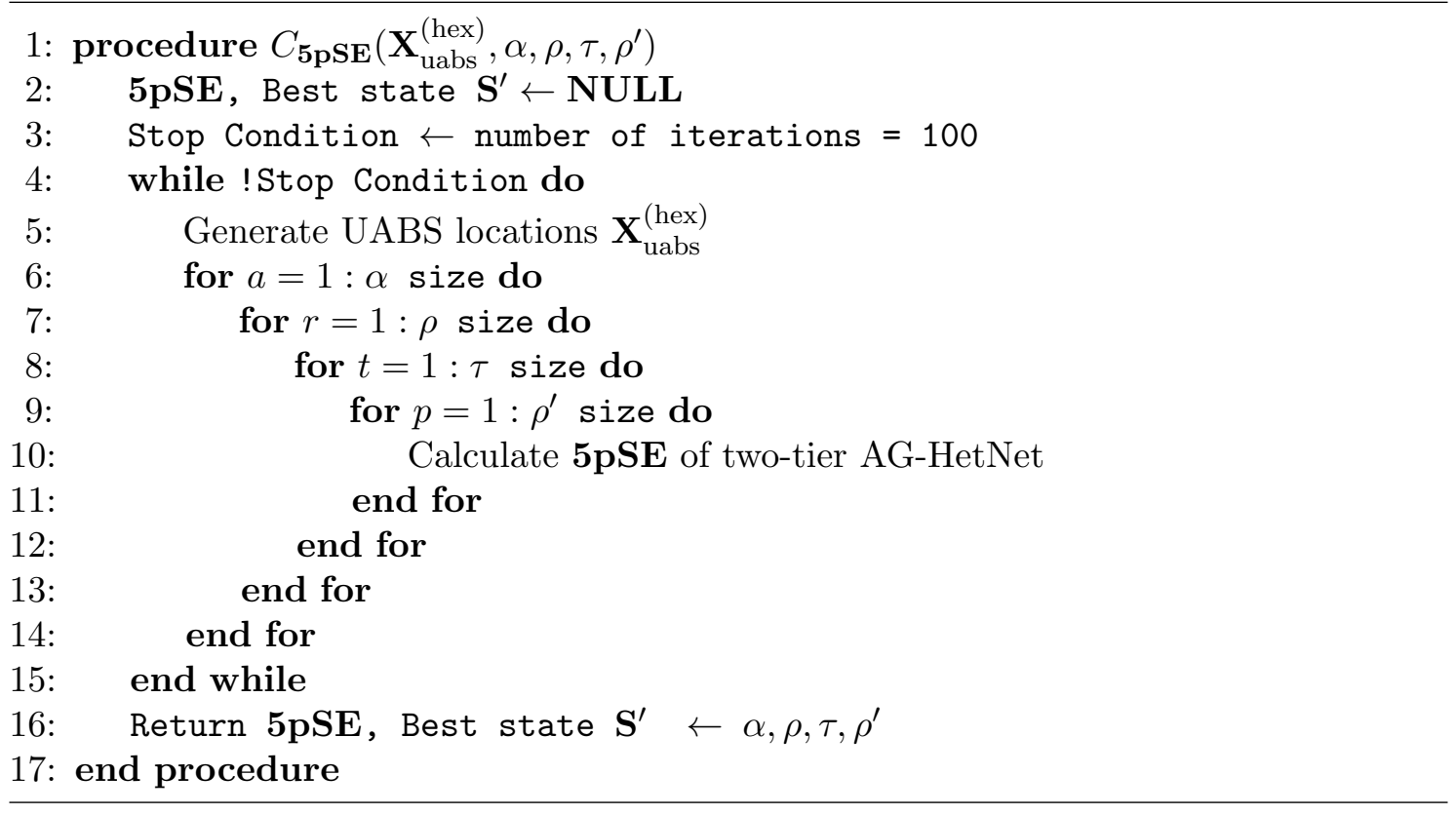

by using a brute force technique as described in the Algorithm 2 which only considers optimization of the ICIC parameters captured through the matrix $\mathbf{S}_{\text {ICIC }}$. In particular, the ICIC parameters that maximize the 5pSE can then be calculated as:

$$
\left[\hat{\alpha}, \hat{\rho}, \hat{\tau}, \hat{\rho}^{\prime}\right]=\arg \max _{\alpha, \rho, \tau, \rho^{\prime}} C_{5 \operatorname{th}}\left(\mathbf{X}_{\text {uabs }}^{\text {(hex) }}, \alpha, \rho, \tau, \rho^{\prime}\right),
$$

where $\mathbf{X}_{\text {uabs }}^{\text {(hex) }}$ are the fixed and known hexagonal locations of the deployed UABSs within the simulation area.

For the MBS locations shown in Fig. 6.1(b), an example outcome of the UABS locations using the hexagonal grid deployment is shown in Fig. 6.6. In the case of loss in PSC network infrastructure, the UABSs can be deployed rapidly on a fixed hexagonal grid as a primary deployment strategy to form new small cells and consequently improve the network coverage. 


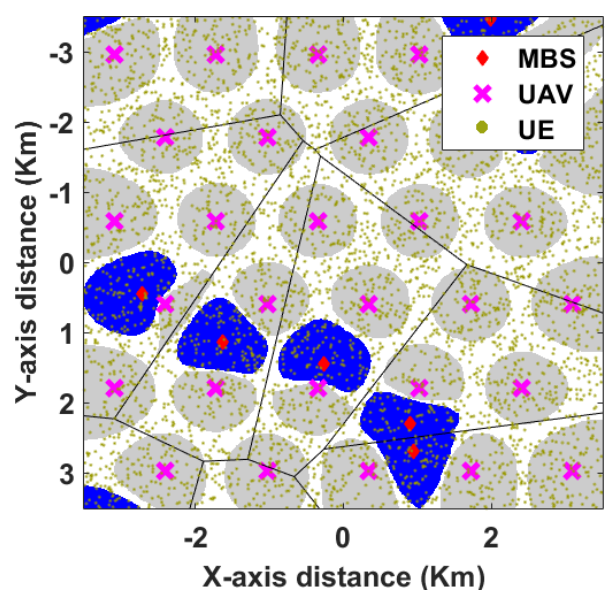

Figure 6.6: Two-tier PSC AG-HetNet after a disaster with UABS deployed on a fixed hexagonal grid (UAV height: $100 \mathrm{~m}$ ).

\subsection{Simulation Results}

In this section, using extensive computer simulations, we compare the 5pSE of a UABS-assisted PSC HetNet with and without ICIC techniques while considering different UABS deployment strategies and path loss models for all the UEs covered by the base stations. Unless otherwise specified, the system parameters for the simulations are set to the values given in Table 6.1.

\subsubsection{5pSE with UABSs Deployed on a Hexagonal Grid}

In the following we will discuss the key 5pSE observations when the UABSs are deployed on a hexagonal grid and utilizing optimized ICIC parameters (see (6.26) and Algorithm 2). In Fig. 6.7 and Fig. 6.9, we plot the variations in 5pSE with respect to CRE while using SPLM and OHPLM, respectively.

\section{5pSE with Simplified Path Loss model}

In Fig. 6.7(a), we plot the 5pSE variation with respect to CRE for no-ICIC mechanism (NIM). In the case of NIM, all the base stations (MBSs and UABSs) always 
Table 6.1: Simulation parameters for two-tier AG-HetNet.

\begin{tabular}{|l|l|}
\hline Parameter & Value \\
\hline MBS and UE intensity & 4 per $\mathrm{km}^{2}$ and $100 \mathrm{per} \mathrm{km}^{2}$ \\
\hline MBS and UABS transmit powers & $46 \mathrm{dBm}$ and $30 \mathrm{dBm}$ \\
\hline Path-loss exponent & 4 \\
\hline Altitude of MBSs & $30 \mathrm{~m}$ \\
\hline Altitude of UABSs & $100 \mathrm{~m}$ \\
\hline Height of UE & $3 \mathrm{~m}$ \\
\hline PSC LTE Band 14 center frequency & $763 \mathrm{MHz}$ for downlink and 793 \\
& $\mathrm{MHz}$ for uplink \\
\hline $\mathrm{d}_{\mathrm{m} n}^{\text {min }}, \mathrm{d}_{\mathrm{mu}}^{\text {min }}$ & $30 \mathrm{~m}, 10 \mathrm{~m}$ \\
\hline Simulation area & $10 \times 10 \mathrm{~km}$ \\
\hline GA population size and generation number & 60 and 100 \\
\hline GA crossover and mutation probabilities & 0.7 and 0.1 \\
\hline Cell range expansion $(\tau)$ in dB & 0 to $15 \mathrm{~dB}$ \\
\hline Power reduction factor for MBS during $(\alpha)$ & 0 to 1 \\
\hline Duty cycle for the transmission of USF $(\beta)$ & 0.5 or $50 \%$ \\
\hline Scheduling threshold for MUEs $(\rho)$ & $20 \mathrm{~dB}$ to $40 \mathrm{~dB}$ \\
\hline Scheduling threshold for UUEs $(\rho \prime)$ & $-20 \mathrm{~dB}$ to $-5 \mathrm{~dB}$ \\
\hline MBS destroyed sequence & $50 \%$ and $97.5 \%$ \\
\hline
\end{tabular}

transmit at full power $\left(P_{\text {mbs }}^{\prime}\right.$ and $\left.P_{\text {uabs }}^{\prime}\right)$. The close evaluation of Fig. 6.7(a), shows that the peak value of $5 \mathrm{pSE}$ for NIM is observed at around $0 \mathrm{~dB}$ CRE. This is because, with no CRE, the number of UEs associated with the UABSs and the interference experienced by these UEs is minimal. Moreover, as the CRE increases, the number of UEs associated with the UABSs increases and so does the interference experienced by these UEs. Hence, with NIM the 5pSE decreases with increasing CRE as seen in Fig. 6.7(a).

The performance of 3GPP Release-10 and Release-11 ICIC techniques in terms of 5pSE and the variation in CRE are plotted in Fig. 6.7(b) and Fig. 6.7(c). As noted in Section 6.1.2, the transmission power during blank subframes at the MBSs for eICIC is 0, and power reduction of the CSFs at the MBSs for FeICIC is $\alpha P_{\mathrm{mbs}}^{\prime}$. Using this understanding, the analysis Fig. 6.7(b) and Fig. 6.7(c) shows that the 5pSE for ICIC techniques at $0 \mathrm{~dB}$ CRE are relatively lower. On the other hand, the 


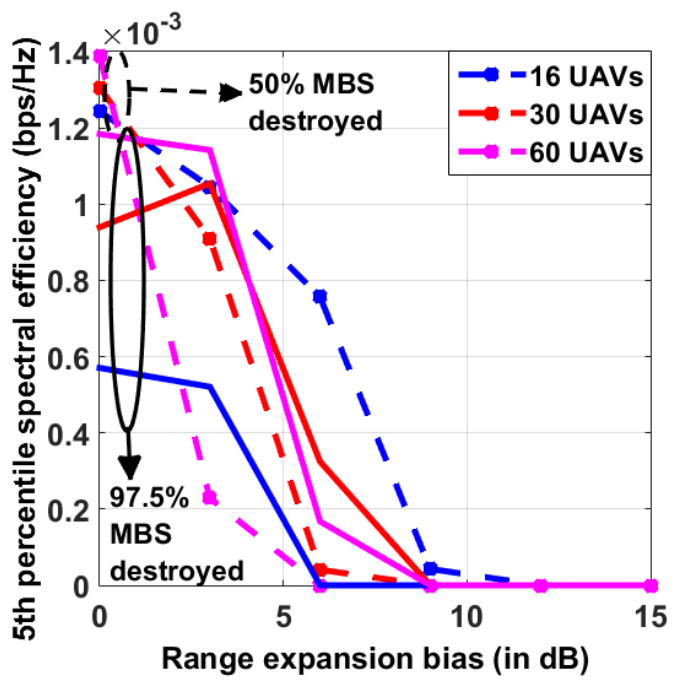

(a) 5 pSE without any ICIC.

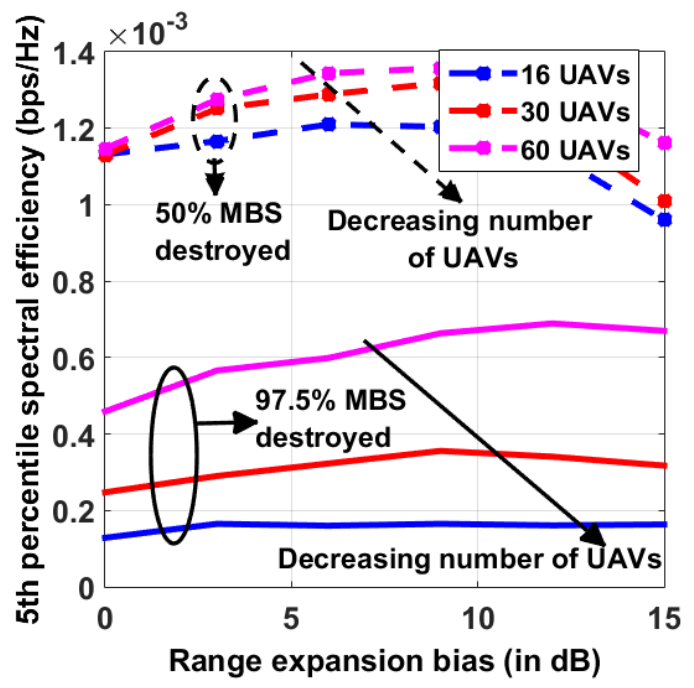

(b) $5 \mathrm{pSE}$ with eICIC.

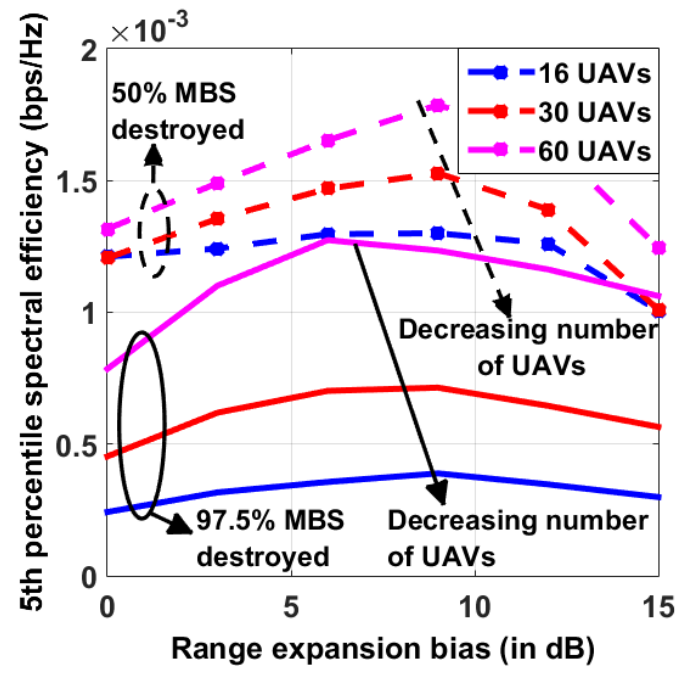

(c) 5 pSE with FeICIC.

Figure 6.7: 5pSE versus CRE for eICIC and FeICIC techniques in two-tier AGHetNet with SPLM (UABSs deployed on a hexagonal grid).

ICIC techniques observe improvement in 5pSE performance with increasing CRE and the peak values of the 5pSE for the ICIC techniques is observed when the CRE is between $6-9 \mathrm{~dB}$. This influence of CRE on the 5pSE for NIM and 3GPP Release-10 and Release-11 ICIC techniques are summarized in Fig. 6.8. 


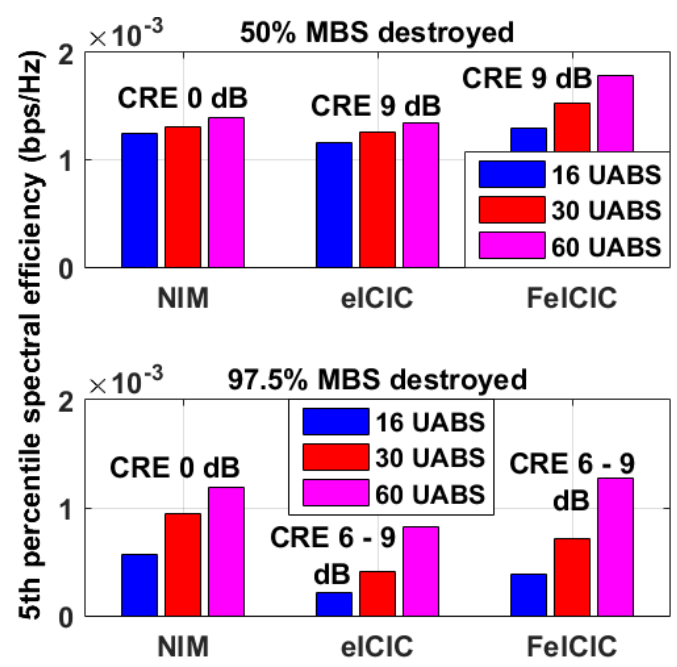

Figure 6.8: Peak observations for the 5pSE with SPLM (UABSs deployed on a hexagonal grid).

\section{5pSE with Okumura-Hata Path Loss Model}

In Fig. 6.9(a), we plot the 5pSE variation with respect to CRE for NIM. In the case of NIM, all the base stations (MBSs and UABSs) always transmit at full power $\left(P_{\mathrm{mbs}}^{\prime}\right.$ and $P_{\text {uabs }}^{\prime}$. The peak value of $5 \mathrm{pSE}$ for NIM is observed at around $3 \mathrm{~dB}$ CRE when $50 \%$ of the MBSs are destroyed. On the other hand, when $97.5 \%$ of the MBSs are destroyed, even though the number of existing MBSs are small and the interference is minimum, the higher path-loss presents higher probability for a cell-edge UE to fall out of coverage area. Moreover, in the absence of any ICIC, using CRE can magnify the impact of interference. Hence, the 5pSE gains with NIM are close to zero.

In Fig. 6.9(b) and Fig. 6.9(c), we plot the variation of 5pSE with respect to CRE for 3GPP Release-10/11 ICIC techniques. The analysis of Fig. 6.9(b) and Fig. 6.9(c) shows that the ICIC techniques observe improvement in 5pSE performance with increasing CRE. When 50\% MBS are destroyed, the 5pSE peak values for the eICIC and FeICIC are observed when the CRE is between $6-9 \mathrm{~dB}$ and $3-6 \mathrm{~dB}$, re- 


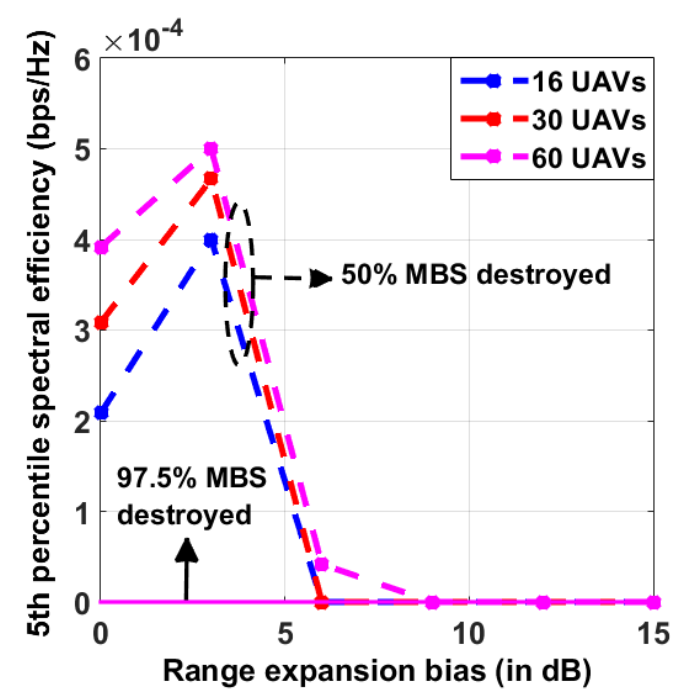

(a) $5 \mathrm{pSE}$ without any ICIC.

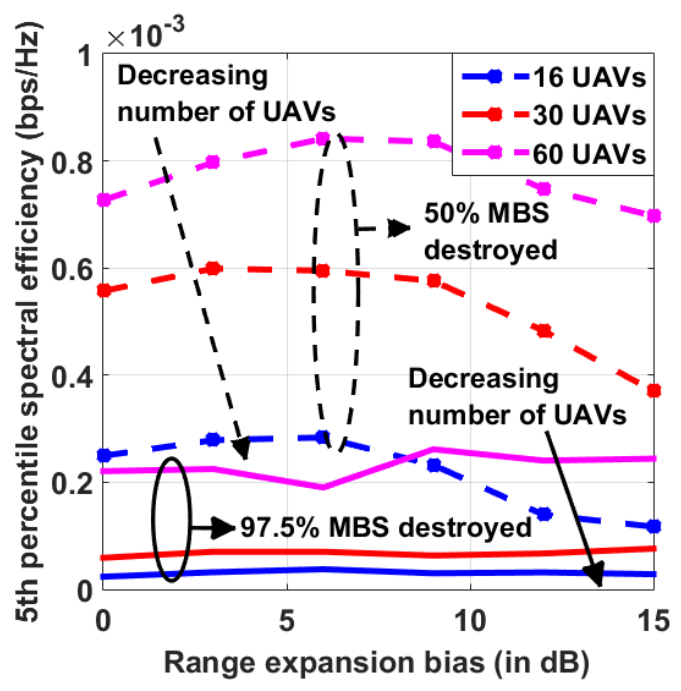

(b) $5 \mathrm{pSE}$ with eICIC.

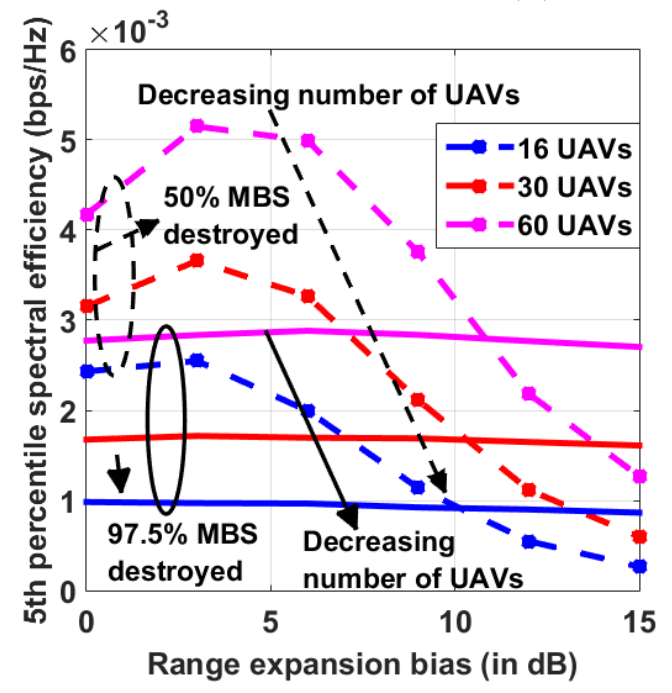

(c) 5 pSE with FeICIC.

Figure 6.9: 5pSE versus $\mathrm{CRE}$ for eICIC and FeICIC technique in two-tier with OHPLM (UABSs deployed on a hexagonal grid).

spectively. When $97.5 \%$ of the MBSs are destroyed, even though the cell-edge UEs observe higher path loss, using the 3GPP Release-10/11 ICIC techniques along with CRE can decrease the probability of cell-edge UE going out of coverage. Thus sustaining the 5pSE of the network as seen in Fig. 6.9(b) and Fig. 6.9(c). Further analysis show that the FeICIC technique observes significant improvement in 


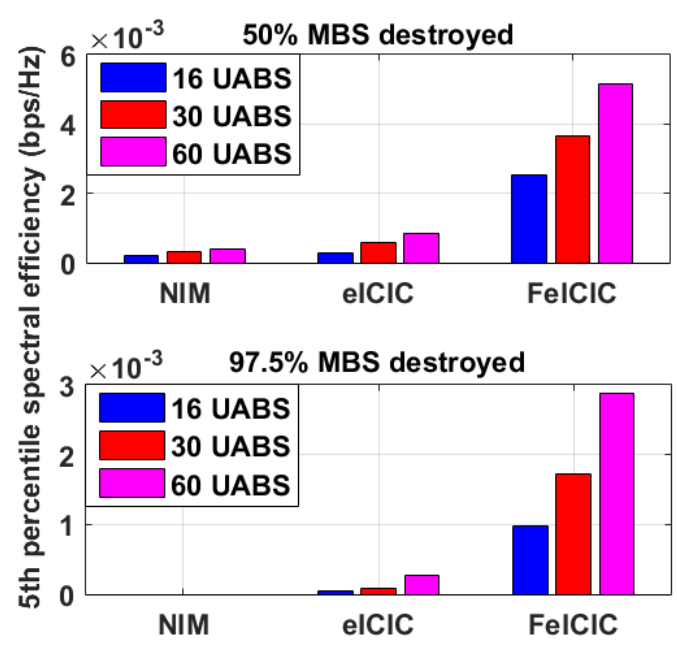

Figure 6.10: Peak observations for the 5pSE with OHPLM (UABSs deployed on a hexagonal grid).

SE performance when compared to NIM and eICIC. This influence of CRE on the 5pSE for NIM and 3GPP Release-10/11 ICIC techniques is summarized in Fig. 6.10.

On comparison of Fig. 6.8 and Fig. 6.10, we observe modest deviation in peak values of 5pSE between NIM, eICIC, and FeICIC with SPLM. This is because UEs experience better SIR with lower path-losses. Whereas, with OHPLM we observe significant deviation in the peak values of 5pSE due to higher path-losses. However, the higher path-losses in OHPLM can be compensated by using modest/higher CRE values and 3GPP Release-10/11 ICIC techniques.

Overall, with hexagonal grid deployment for both the path-loss models, the 5pSE for the network is higher when larger number of UABSs are deployed and when fewer MBSs are destroyed. Also, the 5pSE decreases with the increasing number of destroyed MBSs as seen in Fig. 6.7 and Fig. 6.9. 


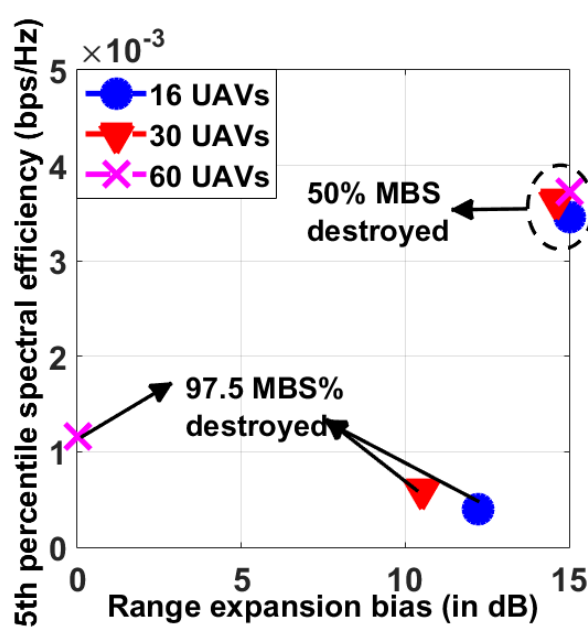

(a) $5 \mathrm{pSE}$ with eICIC.

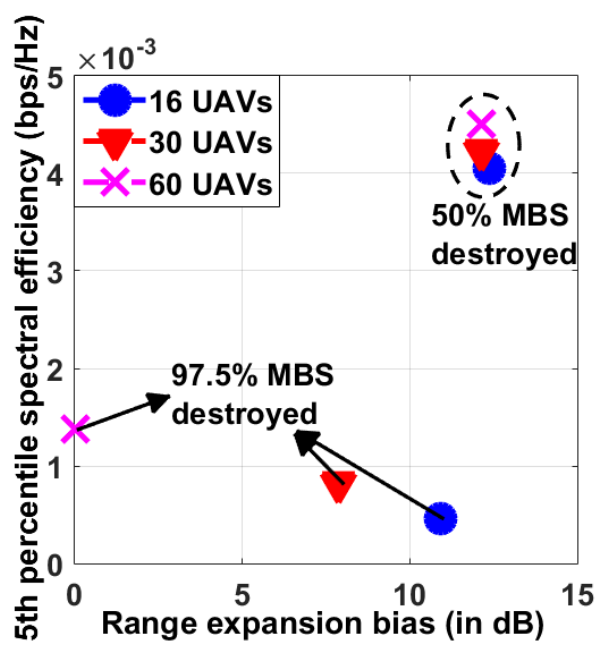

(b) 5 pSE with FeICIC.

Figure 6.11: Peak 5pSE versus optimized CRE for eICIC and FeICIC techniques in two-tier AG-HetNet with SPLM, when the UABS locations and ICIC parameters are optimized using the GA.

\subsubsection{5pSE with GA Based UABS Deployment Optimiza- tion}

In the following, we will discuss the key 5pSE observations, when UABS locations and ICIC parameters optimized through the GA as in (6.25) and Algorithm 1. In Fig. 6.11 and Fig. 6.13, we plot the peak 5pSE for the network using the GA, versus the optimized CRE value while using SPLM and OHPLM, respectively. In the GA based simulations, the optimum CRE value is directly related to the locations of the UABSs with respect to the MBSs, the number of UEs offloaded to the UABSs, and the amount of interference observed by the UEs.

\section{5pSE with Simplified Path Loss Model}

In Fig. 6.11(a) and Fig. 6.11(b), we plot the peak 5pSE with respect to the optimized CRE value for eICIC and FeICIC, respectively, for SPLM. Inspection of Fig. 6.11(a) 
and Fig. 6.11(b) shows higher values of CRE when 50\% of the MBSs are destroyed and implies the presence of substantial interference from these large number of MBSs. Hence, offloading a large number of UEs from MBSs to UABSs with higher values of CRE is necessary for achieving better 5pSE gains.

On the other hand, when most of the infrastructure is destroyed (i.e., when 97.5\% of the MBSs destroyed), the interference observed from the MBSs is limited, and a larger number of UEs need to be served by the UABSs. Therefore, with fewer UABSs deployed, higher CRE is required to serve a larger number of UEs and achieve better 5pSE. On the other hand, when a larger number of UABSs are deployed, smaller values of CRE will result in better 5pSE gains. We record these behavior in Fig. 6.11(a) and Fig. 6.11(b) for eICIC and FeICIC, respectively.

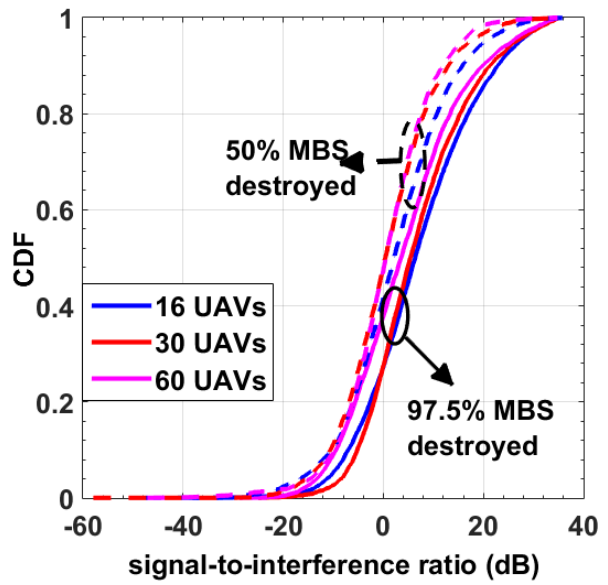

(a) SIR observations for eICIC.

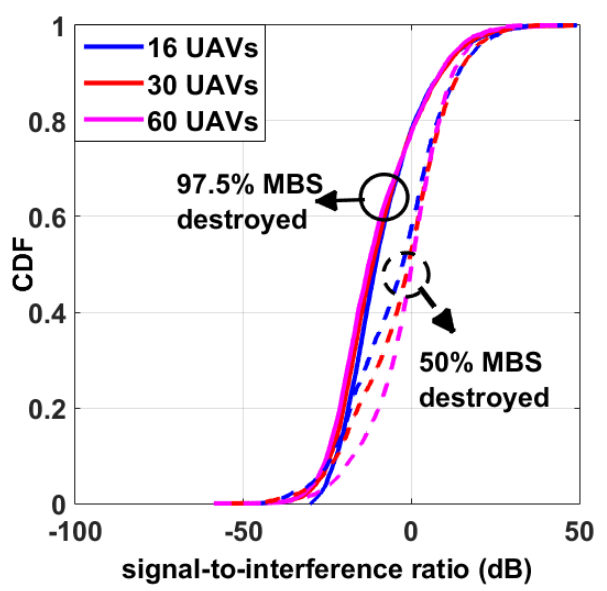

(b) SIR observations FeICIC.

Figure 6.12: SIR observations for eICIC and FeICIC in two-tier HetNet with OHPLM, when UABS locations are optimized using the GA.

\section{5pSE with Okumura-Hata Path Loss model}

Using (6.9)-(6.12), we plot the SIR observations in Fig. 6.12 for 3GPP Release10/11 ICIC techniques. As illustrated in Fig. 6.2(a), the higher path-loss results in lower SIR values as seen in Fig. 6.12. Using this understanding, we inspect the 


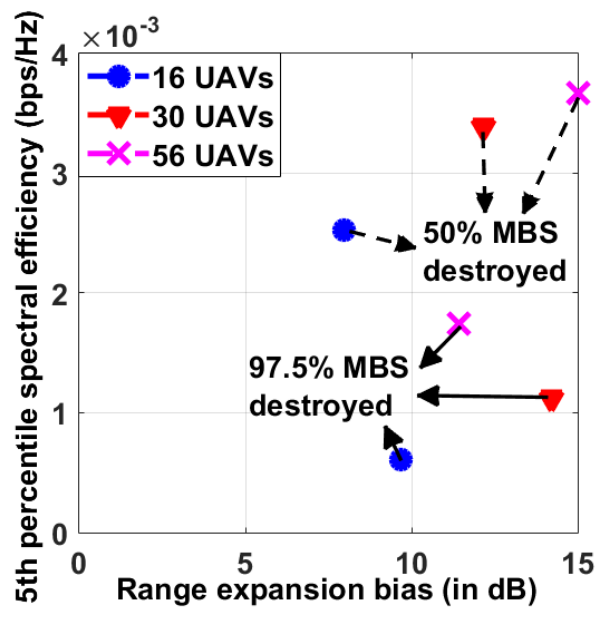

(a) $5 \mathrm{pSE}$ with eICIC.

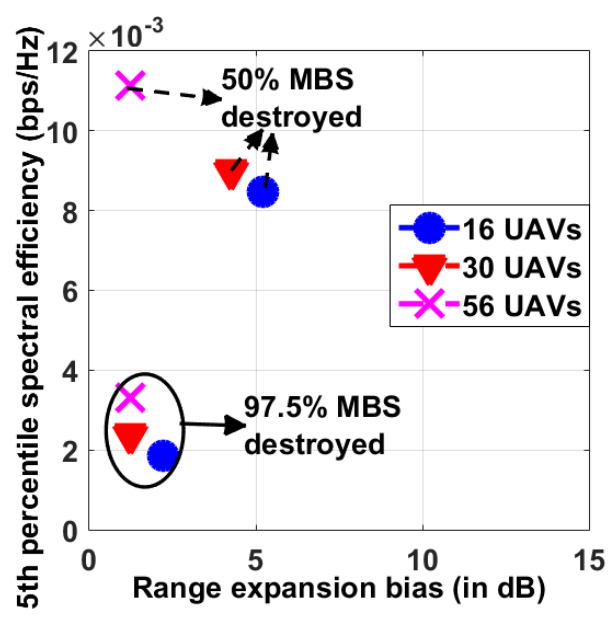

(b) 5 pSE with FeICIC.

Figure 6.13: Peak 5pSE versus optimized CRE for eICIC and FeICIC techniques with OHPLM in two-tier AG-HetNet, when the UABS locations and ICIC parameters are optimized using the GA.

peak 5pSE with respect to the optimized CRE for the eICIC and FeICIC as shown in Fig. 6.13(a) and Fig. 6.13(b), respectively.

With 3GPP Release-10 ABS, higher values of CRE are required for UABSs to compensate for the high path-loss and under-utilization of radio resources by the MBSs in CSF radio subframes. When $50 \%$ and $97.5 \%$ of the MBSs are destroyed, the peak 5pSE values for eICIC are achieved with minimal SIR values, and by offloading a large number of UEs from MBSs to UABSs as seen in Fig. 6.13(a).

On the other hand, with 3GPP Release-11 reduced power subframes (FeICIC), MBSs can establish and maintain connectivity with sufficient number of cell-edge MUEs, while offloading the out-of-coverage UEs to UABSs for better QoS. When $50 \%$ and $97.5 \%$ of the MBSs are destroyed, the peak 5pSE values for FeICIC are achieved with minimal SIR and moderate CRE values as shown in Fig. 6.13(b).

To summarize, using GA for both path-loss models, FeICIC in Release-11 is seen to outperform Release-10 eICIC in terms of the overall 5pSE of the network. When larger number of UABSs are deployed and when fewer MBSs are destroyed, 5pSE of 


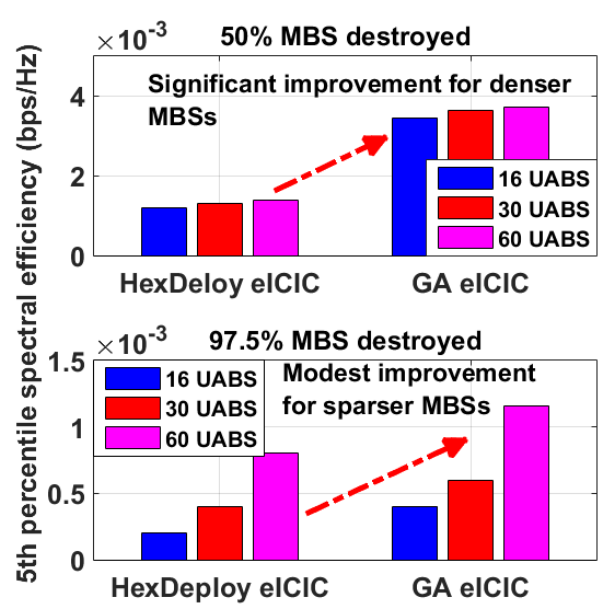

(a) 5pSE with eICIC.

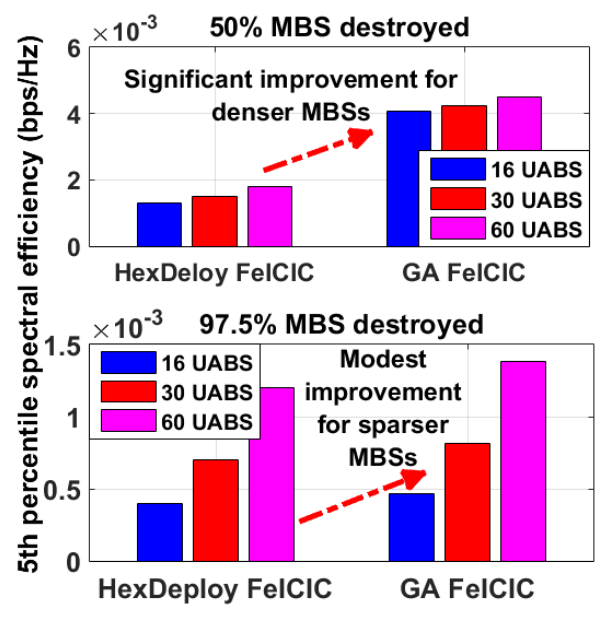

(b) 5pSE with FeICIC.

Figure 6.14: 5pSE comparisons for eICIC and FeICIC with SPLM in two-tier AGHetNet, when the UABS locations are optimized using the GA and when the UABSs are deployed in a fixed hexagonal grid.

the network is higher. On the other hand, the 5pSE decreases with the increasing number of destroyed MBSs as seen in Fig. 6.13.

\subsubsection{Performance Comparison Between Fixed (Hexagonal) and Optimized UABS Deployment with eICIC and FeICIC}

We summarize our key results from earlier simulations in Fig. 6.14 and Fig. 6.15 for both path-loss models and compare the key trade-offs between fixed (hexagonal) deployment and GA based deployment of UABSs.

\section{Influence of SPLM on 5pSE}

In Fig. 6.14, we compare the 5pSE observations with SPLM shown in Fig. 6.7 and

Fig. 6.11. The comparative analysis reveals that UABSs deployment with optimized 


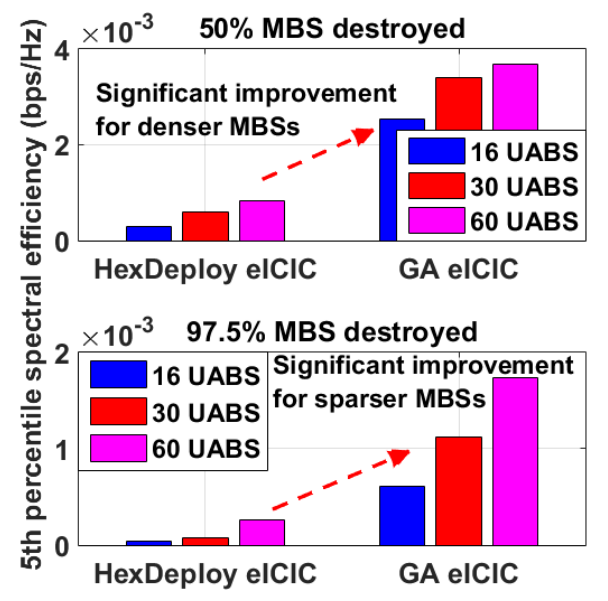

(a) $5 \mathrm{pSE}$ with eICIC.

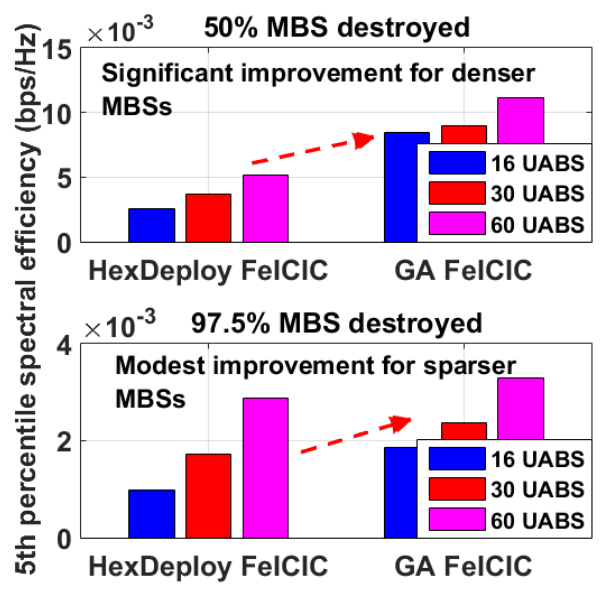

(b) $5 \mathrm{pSE}$ with FeICIC.

Figure 6.15: 5pSE comparisons for eICIC and FeICIC with OHPLM in the two-tier AG-HetNet, when the UABS locations are optimized using the GA and when the UABSs are deployed in a fixed hexagonal grid.

CRE and optimized location provides a better 5pSE than the UABSs deployed on a fixed hexagonal grid. Furthermore, Fig. 6.14 shows that the 5pSE gains from the optimization of UABS locations are more significant when $50 \%$ of the MBSs are destroyed and less significant when $97.5 \%$ of the MBSs are destroyed.

When $50 \%$ MBSs are destroyed, there are still a large number of MBSs present which causes substantial interference. Hence, in such interference driven scenario it is important to optimize the locations of the UABSs, and use of larger number of UABSs to provide significant gains in the 5pSE. On the other hand, with $97.5 \%$ of the MBSs destroyed, the interference from the MBSs is small, and deploying the UABSs on a hexagonal grid will perform close to optimum UABS deployment.

\section{Influence of OHPLM on 5pSE}

In Fig. 6.15, we compare the 5pSE observations with OHPLM shown in Fig. 6.9 and Fig. 6.13. The comparative analysis reveals that UABSs deployment with op- 
timized CRE and optimized location provides a better 5pSE than the UABSs that are deployed on a fixed hexagonal grid.

With eICIC in Release-10, when $50 \%$ and $97.5 \%$ of the MBSs are destroyed, the 5pSE gains from the optimized UABS locations are significant as shown in Fig. 6.15(a). On the other hand, with FeICIC in Release-11, the 5pSE gains from the optimized UABS locations are more significant when $50 \%$ of the MBSs are destroyed as seen in Fig. 6.15(b). However, the difference between the hexagonal deployment and optimized deployment is especially small since the power reduction factor $\alpha$ in the MBS CSFs provides an additional optimization dimension for improving the 5pSE. Use of a larger number of UABSs when $97.5 \%$ of the MBSs are destroyed is shown to provide modest gains in the 5pSE, in contrast to significant gains in the $5 \mathrm{pSE}$ when $50 \%$ of the MBSs are destroyed.

\subsubsection{Comparison of Computation Times for Different UABS}

\section{Deployment Algorithms}

In this subsection, we compare the computation times for the GA and hexagonal grid deployment techniques with ICIC optimization. Using an Intel Core i7-4810 central processing unit operating at $2.8 \mathrm{GHz}, 24 \mathrm{~GB}$ of random access memory, and Monte-Carlo experimental approach, we calculate the mean runtime for the Matlab simulations. In Fig. 6.16, we plot the mean runtime required for calculating the optimal ICIC network parameters and optimized UABS locations using (6.25), Algorithm 1, and the simulation values defined in Table 6.1. Inspection of Fig. 6.16 reveals FeICIC technique requires similar computational time when compared to the eICIC technique for GA based optimization. The main reason for this is that

the large search space for UABS locations, which are common in both FeICIC and 

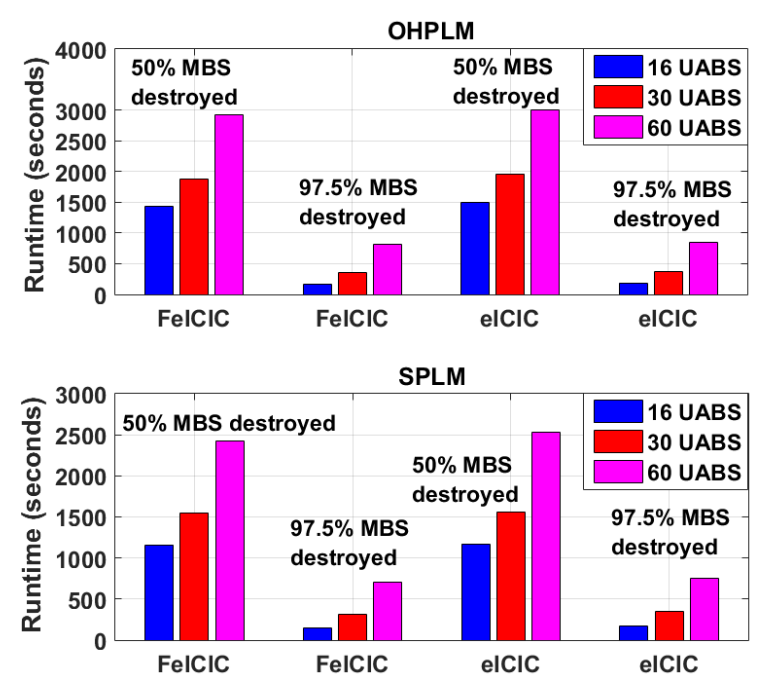

Figure 6.16: GA simulation runtime using FeICIC and eICIC technique with $\mathrm{OH}-$ PLM and SPLM for the proposed two-tier AG-HetNet model.
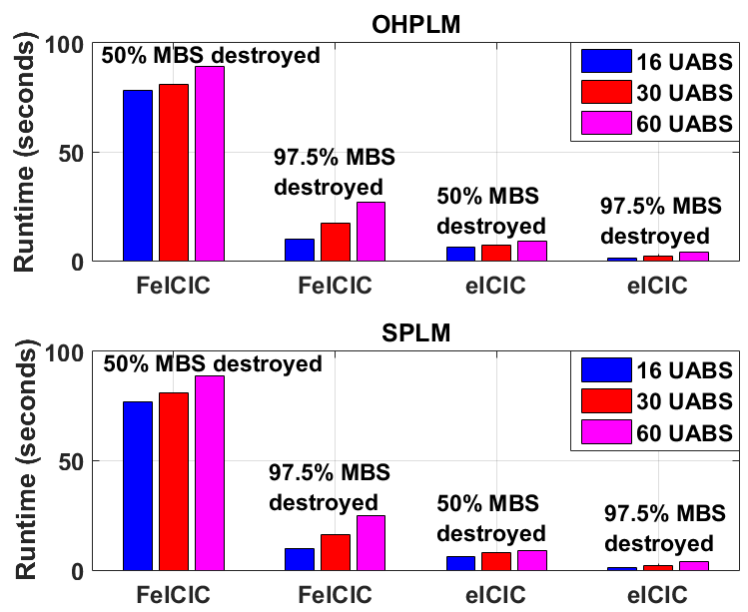

Figure 6.17: Fixed hexagonal grid simulation runtime using FeICIC and eICIC technique with OHPLM and SPLM for the proposed two-tier AG-HetNet model.

eICIC based approaches, dominates the computation time when compared with the optimization of ICIC parameters.

On the other hand, in Fig. 6.17, we plot the mean runtime required for UABS deployment on a hexagonal grid using (6.26), the simulation values defined in Table 6.1, and with fixed step size for the ICIC parameters. Inspection of Fig. 6.17 reveals FeICIC technique requires significantly higher computational time when compared 
to the eICIC technique. The main reason for this behavior is due to additional computation required for optimizing the power reduction factor $\alpha$ for the FeICIC approach. In general, with the GA and the hexagonal grid deployment, when larger number of UABSs are deployed, and larger number of the MBSs are present, the mean runtime is the largest. On the other hand, the mean runtime decreases with smaller number of UABSs deployed and when a smaller number of MBSs are present. Moreover, the comparative analysis of Fig. 6.16 and Fig. 6.17 reveals that optimization (ICIC parameters and UABS locations) using GA requires significantly more computational time when compared to UABSs deployment on a hexagonal grid.

To summarize, the GA is a suitable meta-heuristic technique that relies on bioinspired approach that uses mutations, crossovers, and selections of chromosomes, for finding optimum or close to optimum solution of a search problem. On the other hand, the computational complexity required to optimize the considered UAV deployment optimization problems in real world using the GA techniques require further investigations. 


\section{CHAPTER 7}

\section{HEURISTIC APPROACH FOR JOINTLY OPTIMIZING FEICIC AND UAV LOCATIONS IN MULTI-TIER LTE-ADVANCED PUBLIC SAFETY HETNET}

In order to integrate the UAVs as both AUEs and UABSs, would require a system-level understanding to both modify and extend the existing terrestrial network infrastructure. A vital goal while planning any AG-HetNet is to ensure ubiquitous data coverage with broadband rates. To achieve this goal, we define and simulate an AG-HetNet system model for an urban environment with public safety LTE band class 14, as illustrated in Fig. 1.3. The proposed AG-HetNet model leverages on 3GPP Release 8 CRE, 3GPP Release 10/11 ICIC, 3GPP Release 12 three-dimensional (3D) beamforming (3DBF), and 3GPP Release 15 enhanced support for UAVs. Consequently, to assess the performance of this AG-HetNet, we consider both coverage probability and fifth percentile spectral efficiency as the key performance indicators (KPIs). To maximize the two KPIs of the system model, we jointly optimize the UABS locations in 2D and ICIC and CRE network parameters using a brute-force technique, genetic algorithm (GA), and elitist harmony search algorithm based on the genetic algorithm (eHSGA), while mitigating intercell interference. To reduce the complexity of the optimization algorithms, the deployment height of UABS is not considered during joint optimization. However, we do investigate the impact of UABS height on the overall performance of the wireless network by manually varying the deployment heights.

\subsection{System Model}

We consider a three-tier AG-HetNet deployment, where all the MBS, PBS and UABS locations (in 3D) are captured in matrices $\mathbf{X}_{\mathrm{mbs}} \in \mathbb{R}^{N_{\mathrm{mbs}} \times 3}, \mathbf{X}_{\mathrm{pbs}} \in \mathbb{R}^{N_{\mathrm{pbs}} \times 3}$, 
and $\mathbf{X}_{\text {uabs }} \in \mathbb{R}^{N_{\text {uabs }} \times 3}$, respectively, with $N_{\text {mbs }}, N_{\text {pbs }}$ and $N_{\text {uabs }}$ denoting the number of MBSs, PBSs, and UABSs within the simulation area $\left(\mathrm{A}_{\text {sim }}\right)$. Similarly, the 3D distribution of GUEs and AUEs are respectively captured in matrices $\mathbf{X}_{\text {gue }}$ and $\mathbf{X}_{\text {aue }}$. Assuming a fixed antenna height, the location of wireless nodes MBS, PBS, GUE, and AUE are modeled using a 2D Poisson point process (PPP), with densities $\lambda_{\text {mbs }}$, $\lambda_{\text {pbs }}, \lambda_{\text {gue }}$ and $\lambda_{\text {aue }}$, respectively. On the other hand, UABS locations are either optimized using an eHSGA or GA or deployed on a fixed hexagonal grid at lowaltitude and medium-altitude [282]. The densities and deployment heights each of the wireless nodes are specified in Table 7.2.

Let $N_{\text {ue }}$ be the total numbers of UEs (AUEs + GUEs) to be scheduled, then the nearest distance of an arbitrary $n$th UE from any macrocell of interest (MOI), picocell of interest (POI), and UABS-cell of interest (UOI) is given by $d_{o n}, d_{p n}$, and $d_{u n}$, respectively. Then assuming Nakagami-m fading channel, the reference symbol received power from MOI, POI, and UOI is given by

$$
\begin{aligned}
& R_{\mathrm{mbs}}\left(d_{o n}\right)=\frac{P_{\mathrm{mbs}} A_{E}(\phi, \theta) H}{10^{\varphi\left(d_{o n}\right) / 10}}, \\
& R_{\mathrm{pbs}}\left(d_{p n}\right)=\frac{P_{\mathrm{pbs}} A_{E}(\phi, \theta) H}{10^{\varphi\left(d_{p n}\right) / 10}}, \\
& R_{\text {uabs }}\left(d_{u n}\right)=\frac{P_{\mathrm{uabs}} A_{E}(\phi, \theta) H}{10^{\varphi\left(d_{u n}\right) / 10}},
\end{aligned}
$$

where variables $\varphi\left(d_{o n}\right), \varphi\left(d_{p n}\right)$, and $\varphi\left(d_{u n}\right)$ are path-loss respectively observed from MBS, PBS, and UABS in dB. And random variable $H$ accounts for Nakagamim fading, whose probability density function is given by [234]

$$
f_{N}(\omega, m)=\frac{m^{m} \omega^{m-1}}{\Gamma(m)} \exp (-m \omega)
$$

where $m$ is the shaping parameter, $\omega$ is the channel amplitude and $\Gamma(m)$ is the standard Gamma function given as $\Gamma(m)=\int_{0}^{\infty} \exp (-u) u^{m-1} \mathrm{~d} u$. Through shaping 
parameter $m$, received signal power can be approximated to variable fading conditions. The value $m>1$ approximates to Rician fading along line-of-sight (LOS) and $m=1$ approximates to Rayleigh fading along non-LOS (NLOS). Furthermore, using the definition of zenith $(\theta)$ and azimuth $(\phi)$ spherical angles and spherical unit vectors in a Cartesian coordinate, we define variable $A_{E}(\phi, \theta)$ as the transmitter antenna's 3DBF element and is defined in [283] as

$$
\begin{array}{r}
A_{E}(\phi, \theta)=G_{E, \max }-\min \left\{-\left(A_{H}(\phi)+A_{V}(\theta)\right), A_{m}\right\}, \\
A_{m}-30 \mathrm{~dB}, G_{E, \max }=8 \mathrm{dBi},
\end{array}
$$

where antenna element for horizontal $\left(A_{H}(\phi)\right)$ and vertical $\left(A_{E}(\theta)\right)$ radiation pattern, respectively is given by

$$
\begin{array}{r}
A_{H}(\phi)=-\min \left[12\left(\frac{\phi}{\phi_{3 \mathrm{~dB}}}\right)^{2}, A_{m}\right], \phi_{3 \mathrm{~dB}}=65^{\circ}, \\
A_{E}(\theta)=-\min \left[12\left(\frac{\theta-\theta_{\mathrm{tilt}}}{\theta_{3 \mathrm{~dB}}}\right)^{2}, S L A V\right], \theta_{\mathrm{tilt}}=90^{\circ}, \\
S L A V=30, \theta_{3 \mathrm{~dB}}=65^{\circ} .
\end{array}
$$

Using 3DBF, the power transmission from $\operatorname{MBS}\left(P_{\mathrm{mbs}}\right)$, PBS $\left(P_{\mathrm{pbs}}\right)$, and UABS $\left(P_{\text {uabs }}\right)$ can be controlled at UEs in cell-edge/CRE region. Thus limiting the power transmission into adjacent cells which causes inter-cell interference and subsequently improving signal-to-interference ratio (SIR) of the desired signal [284].

\subsubsection{Path Loss Model}

In an urban environment, based on the type of communication link, i.e., groundto-ground (GTG), any-to-air (ATA), and air-to-ground (ATG) between a UE and 
base-station (BS) of interest, we consider distinct path-loss models for accurate analysis of signal reliability for the proposed AG-HetNet.

\section{GTG Communication Link}

We consider Okumura-Hata Path Loss (OHPL) while estimating the GTG communication link between GUE and terrestrial MBS and PBS. The OHPL is better suited to an urban terrestrial environment, in which the base-station height does not vary $[36,274]$ significantly. When a GUE camps on a terrestrial base-station of interest (MOI or POI), OHPL is given by

$$
\varphi(d)=74.52+26.16 \log \left(f_{\mathrm{c}}\right)-20.37 \log \left(h_{\mathrm{bs}}\right)-3.2\left(\log \left(11.75 h_{\text {gue }}\right)\right)^{2}+38.35 \log (d)
$$

where $f_{\mathrm{c}}$ is the carrier frequency in $\mathrm{MHz}, h_{\text {gue }}$ is the height of GUE in meter, and $h_{\mathrm{bs}}$ is the height of terrestrial base-station in meter i.e., height of MBS is given by $h_{\mathrm{mbs}}$ and PBS by $h_{\mathrm{pbs}}$.

\section{ATA Communication Link}

Whenever, an AUE camps on any nearest base-station, we consider a 3D channel model for an urban-macro with aerial (UMa-AV) scenario defined in 3GPP Release15 [285]. The UMa-AV LOS and NLOS path loss, respectively are given by

$$
\varphi(d)=\left\{\begin{aligned}
\varphi^{\operatorname{LOS}}(d) & =28.0+22 \log _{10}\left(d_{3 \mathrm{D}}\right)+20 \log _{10}\left(f_{\mathrm{c}}\right) \\
\varphi^{\operatorname{NLOS}}(d) & =-17.5+\left(46-7 \log _{10}\left(h_{\mathrm{aue}}\right)\right) 10 \log _{10}\left(d_{3 \mathrm{D}}\right) \\
& +20 \log _{10}\left(\frac{40 \pi f_{\mathrm{c}}}{3}\right)
\end{aligned}\right.
$$

where $f_{\mathrm{c}}$ is the carrier frequency in $\mathrm{MHz}, d_{3 \mathrm{D}}$ is the $3 \mathrm{D}$ distance between AUE and the base-station of interest, and $h_{\text {aue }}$ is the height of AUE in meter such that $22.5 \mathrm{~m}<\mathrm{h}_{\text {aue }} \leq 300 \mathrm{~m}$ for $\varphi^{\operatorname{LOS}}(d)$ and $10.0 \mathrm{~m}<\mathrm{h}_{\text {aue }} \leq 100 \mathrm{~m}$ for $\varphi^{\mathrm{NLOS}}(d)$. 
The LOS probabilities for the ATA communication link defined in 3GPP Release15 [285] is given

$$
\mathbb{P}_{\mathrm{LOS}}(\varphi)= \begin{cases}1, & d_{2 \mathrm{D}} \leq d_{1} \\ \frac{d_{1}}{d_{2 \mathrm{D}}}+\exp \left(\frac{-d_{2 \mathrm{D}}}{p_{1}}\right)\left(1-\frac{d_{1}}{d_{2 \mathrm{D}}}\right), & d_{2 \mathrm{D}}>d_{1}\end{cases}
$$

where is $d_{2 \mathrm{D}}$ is the $2 \mathrm{D}$ distance between AUE and the base-station of interest such that $d_{2 \mathrm{D}} \leq 4 \mathrm{~km}$, and the factors $p_{1}$ and $d_{1}$ (in meters) are given by

$$
\begin{aligned}
& p_{1}=4300 \log _{10}\left(h_{\text {aue }}\right)-3800, \\
& d_{1}=\max \left(460 \log _{10}\left(h_{\text {aue }}\right)-700,18\right) .
\end{aligned}
$$

Using this model, we calculate the average path loss over the probabilities of LOS and NLOS communication link between AUE and the camping base-station. Then using (7.7) and (7.8), the average path loss is given by

$$
\mathrm{PL}_{\mathrm{avg}}=\mathbb{P}_{\mathrm{LOS}} \times \varphi^{\mathrm{LOS}}+\left(1-\mathbb{P}_{\mathrm{LOS}}\right) \times \varphi^{\mathrm{NLOS}}
$$

\section{ATG Communication Link}

Whenever, a GUE camps on a UOI, we consider a more conventionally used LOS/NLOS path loss model defined in the literature $[217,286,287]$ and is given by

$$
\varphi_{\mathrm{uabs}}(d)=\prod_{x=0}^{y}\left[1-\exp \left(-\frac{\left[h_{\mathrm{uabs}}-\frac{(x+1 / 2)\left(h_{\mathrm{uabs}}-h_{\mathrm{gue}}\right)}{y+1}\right]^{2}}{2 \Omega^{2}}\right)\right],
$$

where $h_{\mathrm{uabs}}$ is the deployment height of UABS, $y=$ floor $(r \sqrt{\zeta \xi}-1)$, r is the ground distance between the UABS and GUE, $\zeta$ is the ratio of built-up land area to the total land area, $\xi$ is the mean number of buildings per unit area (buildings $/ \mathrm{km}^{2}$ ), and $\Omega$ characterizes the building height (denoted by $H_{\mathrm{B}}$ ) distribution in meters and is based on a Rayleigh distribution: $f\left(H_{\mathrm{B}}\right)=\frac{H_{\mathrm{B}}}{\Omega^{2}} \exp \left(\frac{-H_{\mathrm{B}}^{2}}{2 \Omega^{2}}\right)$. Furthermore, we consider LOS probability $\mathbb{P}_{\mathrm{LOS}}\left(\varphi_{\text {uabs }}\right)$ as a continuous function of $\theta$ and environment factors. By 


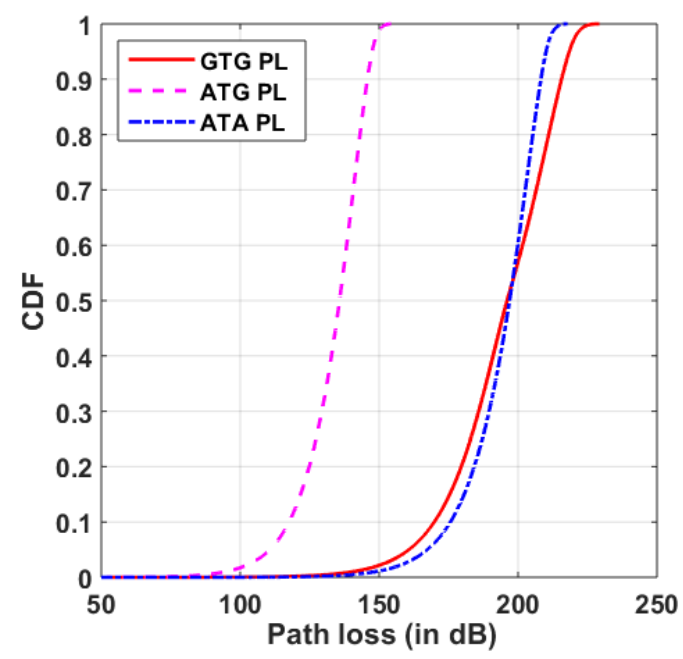

Figure 7.1: The CDF of path loss observed for the communication link between UEs (AUE and GUE) and base-stations (MBS, PBS, and UABS) in a three-tier AG-HetNet.

approximating environment factors to a simple modified Sigmoid function (S-curve), the simplified LOS probability is given by

$$
\mathbb{P}_{\text {LOS }}\left(\varphi_{\text {uabs }}, \theta\right)=\frac{1}{1+a \exp (-b[\theta-a])}
$$

where $a$ and $b$ are the S-curve parameters.

Fig. 7.1 illustrates the empirical path loss cumulative distribution functions (CDFs), calculated for all distances between base stations $\left(\mathbf{X}_{\mathrm{mbs}}, \mathbf{X}_{\mathrm{pbs}}\right.$, and $\left.\mathbf{X}_{\mathrm{uabs}}\right)$ and UEs $\left(\mathbf{X}_{\text {gue }}\right.$ and $\left.\mathbf{X}_{\text {aue }}\right)$, using conditions defined in previous paragraph. Inspection of Fig. 7.1 reveals that the maximum allowable path loss is diverse for GTG, ATG, and ATA communication links. This variation is primarily due to the environmental factors and LOS/NLOS probability of communication link. Nevertheless, maximum allowable path-loss for the models used in GTG, ATA, and ATG link is approximately $255 \mathrm{~dB}, 216 \mathrm{~dB}$, and $154 \mathrm{~dB}$, respectively. 


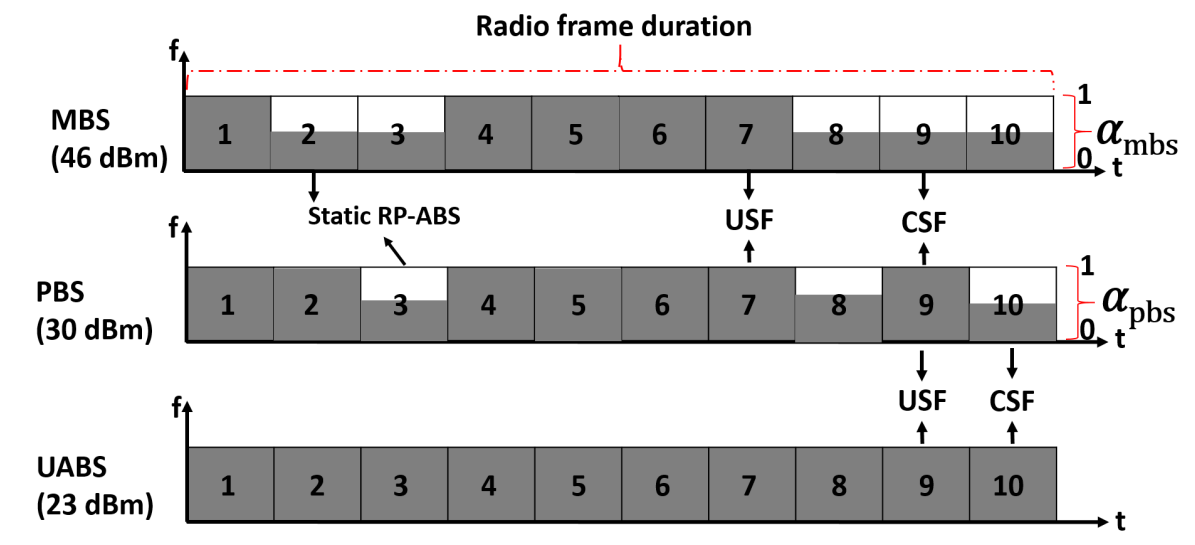

Figure 7.2: The proposed three-tier reduced power USF/CSF LTE subframes of MBS, PBS, and UABS. Certain UABS subframes are protected from both MBS and PBS, while certain PBS subframes are protected from MBS.

\subsubsection{Spectral Efficiency with 3GPP Release-10/11 ICIC}

Due to their low transmission power, the small cells such as the PBS and UABS are unable to associate a substantial number of UEs compared to that of MBSs. Therefore, we consider the CRE technique defined in 3GPP Release- 8 at small cells to extend the network coverage and increase capacity by offloading traffic from congested cells. Although an adverse side effect of CRE includes increased interference at UEs in the cell-edge or CRE region. To address this intercell interference, both MBS and PBS are capable of using ICIC techniques defined in 3GPP Release10/11 [36], wherein both MBS and PBS can transmit radio frames at reduced power levels as shown in Fig.7.2.

The radio subframes with reduced power are termed as coordinated subframes (CSF) and full power as uncoordinated subframes (USF). The power reduction factor of radio subframes at MBS is given by $\alpha_{\mathrm{mbs}}$ and $\alpha_{\mathrm{pbs}}$ at PBS. In particular, $\alpha_{\mathrm{mbs}}=$ $\alpha_{\mathrm{pbs}}=0$ corresponds to Release-10 almost blank subframes (ABS) eICIC, $\alpha_{\mathrm{mbs}}=$ $\alpha_{\mathrm{pbs}}=1$ corresponds to no ICIC, and otherwise corresponds to reduced power FeICIC defined in Release-11. As illustrated in Fig.7.2, using reduced power FeICIC, 
Table 7.1: Signal-to-interference ratio and spectral efficiency definitions.

\begin{tabular}{|c|c|}
\hline Signal-to-interference ratio & $\mathrm{SE}$ in USF / CSF radio frames \\
\hline $\begin{array}{l}\frac{R_{\mathrm{mbs}}\left(d_{o n}\right)}{\Gamma_{\mathrm{usf}}^{\mathrm{mbs}}}=\frac{\alpha}{R_{\mathrm{pbs}}\left(d_{p n}\right)+R_{\mathrm{uabs}}\left(d_{u n}\right)+\mathbf{I}_{\mathrm{agg}}} \\
\Gamma_{\mathrm{csf}}^{\mathrm{mbs}}=\frac{\alpha R_{\mathrm{mbs}}\left(d_{o n}\right)}{\alpha_{\mathrm{pbs}} R_{\mathrm{pbs}}\left(d_{p n}\right)+R_{\text {uabs }}\left(d_{u n}\right)+\mathbf{I}_{\mathrm{agg}}}\end{array}$ & $\begin{aligned} C_{\mathrm{usf}}^{\mathrm{mbs}} & =\frac{\beta_{\mathrm{mbs}} \log _{2}\left(1+\Gamma_{\mathrm{usf}}^{\mathrm{mbs}}\right)}{N_{\mathrm{usf}}^{\mathrm{mbs}}} \\
C_{\mathrm{csf}}^{\mathrm{mbs}} & =\frac{\left(1-\beta_{\mathrm{mbs}}\right) \log _{2}\left(1+\Gamma_{\mathrm{csf}}^{\mathrm{mbs}}\right)}{N_{\mathrm{csf}}^{\mathrm{mbs}}}\end{aligned}$ \\
\hline $\begin{aligned} \Gamma_{\mathrm{usf}}^{\mathrm{pbs}} & =\frac{R_{\mathrm{pbs}}\left(d_{p n}\right)}{R_{\mathrm{mbs}}\left(d_{o n}\right)+R_{\mathrm{uabs}}\left(d_{u n}\right)+\mathbf{I}_{\mathrm{agg}}} \\
\Gamma_{\mathrm{csf}}^{\mathrm{pbs}} & =\frac{\alpha_{\mathrm{pbs}} R_{\mathrm{pbs}}\left(d_{p n}\right)}{\alpha R_{\mathrm{mbs}}\left(d_{o n}\right)+R_{\mathrm{uabs}}\left(d_{u n}\right)+\mathbf{I}_{\mathrm{agg}}}\end{aligned}$ & $\begin{array}{l}C_{\mathrm{usf}}^{\mathrm{pbs}}=\frac{\beta_{\mathrm{pbs}} \log _{2}\left(1+\Gamma_{\mathrm{usf}}^{\mathrm{pbs}}\right)}{N_{\mathrm{usf}}^{\mathrm{pbs}}} \\
C_{\mathrm{csf}}^{\mathrm{uabs}}=\frac{\left(1-\beta_{\mathrm{pbs}}\right) \log _{2}\left(1+\Gamma_{\mathrm{csf}}^{\mathrm{uabs}}\right)}{N_{\text {csf }}^{\mathrm{uue}}}\end{array}$ \\
\hline $\begin{array}{l}\Gamma_{\mathrm{usf}}^{\mathrm{uabs}}=\frac{R_{\mathrm{uabs}}\left(d_{u n}\right)}{R_{\mathrm{mbs}}\left(d_{o n}\right)+R_{\mathrm{pbs}}\left(d_{p n}\right)+\mathbf{I}_{\mathrm{agg}}} \\
\Gamma_{\mathrm{csf}}^{\mathrm{uabs}}=\frac{R_{\mathrm{uabs}}\left(d_{u n}\right)}{\alpha R_{\mathrm{mbs}}\left(d_{o n}\right)+\alpha_{\mathrm{pbs}} R_{\mathrm{pbs}}\left(d_{p n}\right)+\mathbf{I}_{\mathrm{agg}}}\end{array}$ & $\begin{array}{l}C_{\mathrm{usf}}^{\mathrm{mbs}}=\frac{\left(\beta_{\mathrm{mbs}}+\beta_{\mathrm{pbs}}\right) \log _{2}\left(1+\Gamma_{\mathrm{usf}}^{\mathrm{uabs}}\right)}{N_{\mathrm{usf}}^{\text {uae }}} \\
C_{\mathrm{csf}}^{\mathrm{uabs}}=\frac{\left(2-\left(\beta_{\mathrm{mbs}}+\beta_{\mathrm{pbs}}\right)\right) \log _{2}\left(1+\Gamma_{\mathrm{csf}}^{\mathrm{uabs}}\right)}{N_{\mathrm{csf}}^{\text {uue }}}\end{array}$ \\
\hline
\end{tabular}

we protect certain UABS subframes from both MBS and PBS, while certain PBS subframes are protected from MBS. We coordinate the USF/CSF duty cycle using $\beta_{\mathrm{mbs}}$ and $\left(1-\beta_{\mathrm{mbs}}\right)$ at MBS and $\beta_{\mathrm{pbs}}$ and $\left(1-\beta_{\mathrm{pbs}}\right)$ at PBS. The proposed AG-HetNet model assumes that the power reduction pattern and radio subframes duty cycle is shared via the X2 interface, which is a logical interface between the base-stations.

Although applying the ICIC technique at each base-station reduces the intercell interference with adjacent cells, it also reduces the desired SIR at the scheduled UEs. Therefore, to improve the desired SIR, we consider the 3DBF at each transmitting base-station to restrict the beamforming and power transmission to the location of scheduled UE [284].

Given the ICIC framework in 3GPP LTE-Advanced and using a three-tier reduced power USF/CSF structure given in Fig. 7.2, we define the SIR experienced by a $n$th arbitrary UE scheduled in USF/CSF of MOI, POI, and UOI by following an approach similar to that given in [36]. Then, let $\Gamma_{\mathrm{usf}}^{\mathrm{mbs}}, \Gamma_{\mathrm{csf}}^{\mathrm{mbs}}, \Gamma_{\mathrm{usf}}^{\mathrm{pbs}}, \Gamma_{\mathrm{csf}}^{\mathrm{pbs}}, \Gamma_{\mathrm{usf}}^{\mathrm{uabs}}$, and $\Gamma_{\mathrm{csf}}^{\mathrm{uabs}}$ be the SIRs for the UE scheduled in the USF/CSF of MOI, POI, and UOI, respectively and is defined in Table 7.1 . Wherein, $\mathbf{I}_{\text {agg }}$ is the aggregate interference at the UE from all the base-stations, except the MOI, POI, and UOI. 


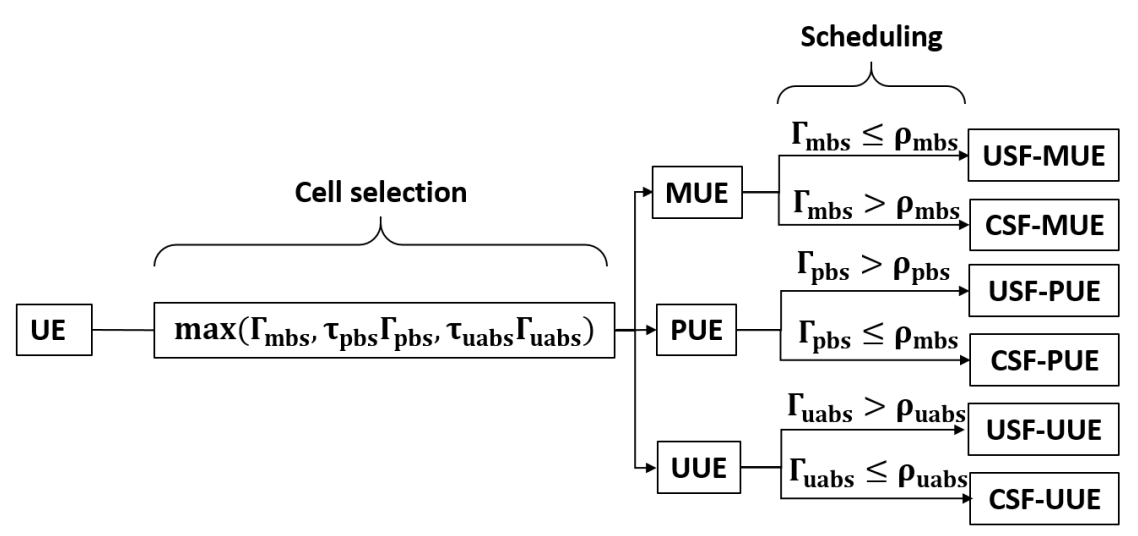

Figure 7.3: Cell selection and UE association in USF/CSF subframes of MBS, PBS, and UABS in three-tier AG-HetNet.

The cell selection process relies on the definition of MOI, POI, and UOI SIRs given in Table 7.1, as well as the CRE $\tau_{\text {pbs }}$ at PBSs and $\tau_{\text {uabs }}$ at UABSs. Using positive biased CRE $\tau_{\text {pbs }}$ at PBSs and $\tau_{\text {uabs }}$ at UABSs, the small cells can further expand their SIR coverage. Consequently, during the cell selection process, a UE always camps on an MOI/POI/UOI that yields the best SIR. After cell selection, an MBS-UE (MUE), PBS-UE (PUE), and UABS-UE (UUE) would be scheduled in either USF/CSF radio subframes based on the scheduling threshold of MBS $\left(\rho_{\text {mbs }}\right)$ ), PBS $\left(\rho_{\text {pbs }}\right)$, and UABS $\left(\rho_{\text {uabs }}\right)$. This strategy of cell selection and UE scheduling in $\mathrm{USF} / \mathrm{CSF}$ of MOI/POI/UOI is similar to that of [36] and is summarized in Fig. 7.3.

Once the $n$th arbitrary UE is assigned to an MOI/POI/UOI and scheduled within the USF/CSF, then using the SIR definitions, the SE of a UE scheduled in the three-tier USF/CSF subframes is defined by $C_{\mathrm{usf}}^{\mathrm{mbs}}, C_{\mathrm{csf}}^{\mathrm{mbs}}, C_{\mathrm{usf}}^{\mathrm{pbs}}, C_{\mathrm{csf}}^{\mathrm{pbs}}, C_{\mathrm{usf}}^{\mathrm{uabs}}$, and $C_{\mathrm{csf}}^{\text {uabs }}$ and is given in Table 7.1. Where $N_{\mathrm{usf}}^{\text {mue }}, N_{\mathrm{csf}}^{\text {mue }}, N_{\mathrm{usf}}^{\text {pue }}, N_{\mathrm{csf}}^{\mathrm{pue}}, N_{\mathrm{usf}}^{\text {uue }}$, and $N_{\mathrm{csf}}^{\text {uue }}$ are the number of MBS-UE, PBS-UE, and UABS-UE scheduled in USF/CSF of the MBS/PBS/UABS. 


\subsection{UABS Locations and ICIC Parameter Optimization in AG-HetNet}

In the proposed system model of three-tier AG-HetNet, 5pSE corresponds to the worst fifth percentile UE capacity amongst all of the scheduled UEs. On the other hand, we define the coverage probability of the network as the percentage of an area having broadband rates and capacity larger than a threshold of $T_{C_{\mathrm{SE}}}$. The primary goal of this simulation study is to maximize these two KPIs while obtaining the best fit ICIC network configuration and optimal UABS locations using a brute force algorithm, genetic algorithm, and elitist harmony search based on the genetic algorithm.

Consider individual locations $\left(x_{i}, y_{i}\right)$ of each UABS $i \in\left\{1,2, \ldots, N_{\text {uabs }}\right\}$ and $\mathbf{X}_{\text {uabs }}$ would be the matrix representing these locations in 3D deployed over a geographical area of interest. The UABSs are placed within the rectangular simulation area regardless of the existing MBS $\left(\mathbf{X}_{\mathrm{mbs}}\right)$ and PBS locations $\left(\mathbf{X}_{\mathrm{pbs}}\right)$. Given the locations of base-station $\left(\mathbf{X}_{\mathrm{mbs}}, \mathbf{X}_{\mathrm{pbs}}\right.$, and $\left.\mathbf{X}_{\mathrm{uabs}}\right)$, we capture individual ICIC parameters for each MBS in a matrix $\mathbf{S}_{\mathrm{mbs}}^{\mathrm{ICIC}}=\left[\boldsymbol{\alpha}_{\boldsymbol{m b s}}, \boldsymbol{\beta}_{\boldsymbol{m b s}}, \boldsymbol{\rho}_{\boldsymbol{m} \boldsymbol{b} \boldsymbol{s}}\right] \in \mathbb{R}^{N_{\mathrm{mbs}} \times 3}$, individual ICIC parameters for each PBS in matrix $\mathbf{S}_{\mathrm{pbs}}^{\mathrm{ICIC}}=\left[\boldsymbol{\alpha}_{\boldsymbol{p} \boldsymbol{b} \boldsymbol{s}}, \boldsymbol{\beta}_{\boldsymbol{p b s}}, \boldsymbol{\rho}_{\boldsymbol{p} \boldsymbol{b} \boldsymbol{s}}, \boldsymbol{\tau}_{\boldsymbol{p} \boldsymbol{b} \boldsymbol{s}}\right] \in \mathbb{R}^{N_{\mathrm{pbs}} \times 4}$, and $\mathbf{S}_{\text {uabs }}^{\text {ICIC }}=\left[\boldsymbol{\tau}_{\text {uabs }}, \boldsymbol{\rho}_{\text {uabs }}\right] \in \mathbb{R}^{N_{\text {uabs }} \times 2}$ is a matrix that captures individual ICIC parameters for each UABS. The vectors $\boldsymbol{\alpha}_{\boldsymbol{m} \boldsymbol{b} \boldsymbol{s}}=\left[\alpha_{1}, \ldots, \alpha_{N_{\mathrm{mbs}}}\right]^{T}, \boldsymbol{\beta}_{\boldsymbol{m} \boldsymbol{b} \boldsymbol{s}}=\left[\beta_{1}, \ldots, \beta_{N_{\mathrm{mbs}}}\right]^{T}$, and $\boldsymbol{\rho}_{\boldsymbol{m b s}}=\left[\rho_{1}, \ldots, \rho_{N_{\mathrm{mbs}}}\right]^{T}$ capture the power reduction factors, USF duty cycle, and scheduling thresholds, respectively, for each MBS. On the other hand, for each PBS, $\boldsymbol{\alpha}_{\boldsymbol{p} \boldsymbol{b} \boldsymbol{s}}=\left[\alpha_{1}, \ldots, \alpha_{N_{\mathrm{pbs}}}\right]^{T}, \boldsymbol{\beta}_{\boldsymbol{p} \boldsymbol{b} \boldsymbol{s}}=\left[\beta_{1}, \ldots, \beta_{N_{\mathrm{pbs}}}\right]^{T}, \boldsymbol{\rho}_{\boldsymbol{p} \boldsymbol{b} \boldsymbol{s}}=\left[\rho_{1}, \ldots, \rho_{N_{\mathrm{pbs}}}\right]^{T}$, and $\boldsymbol{\tau}_{\boldsymbol{p} \boldsymbol{b s}}=\left[\tau_{1}, \ldots, \tau_{N_{\mathrm{pbs}}}\right]^{T}$ capture the power reduction, USF duty cycle, scheduling threshold, and range expansion, respectively. Whereas, $\boldsymbol{\rho}_{\boldsymbol{u a b s}}=\left[\rho_{1}, \ldots, \rho_{N_{\mathrm{uabs}}}\right]^{T}$ and $\boldsymbol{\tau}_{\text {uabs }}=\left[\tau_{1}, \ldots, \tau_{N_{\text {uabs }}}\right]^{T}$ capture the scheduling threshold and range expansion, re- 


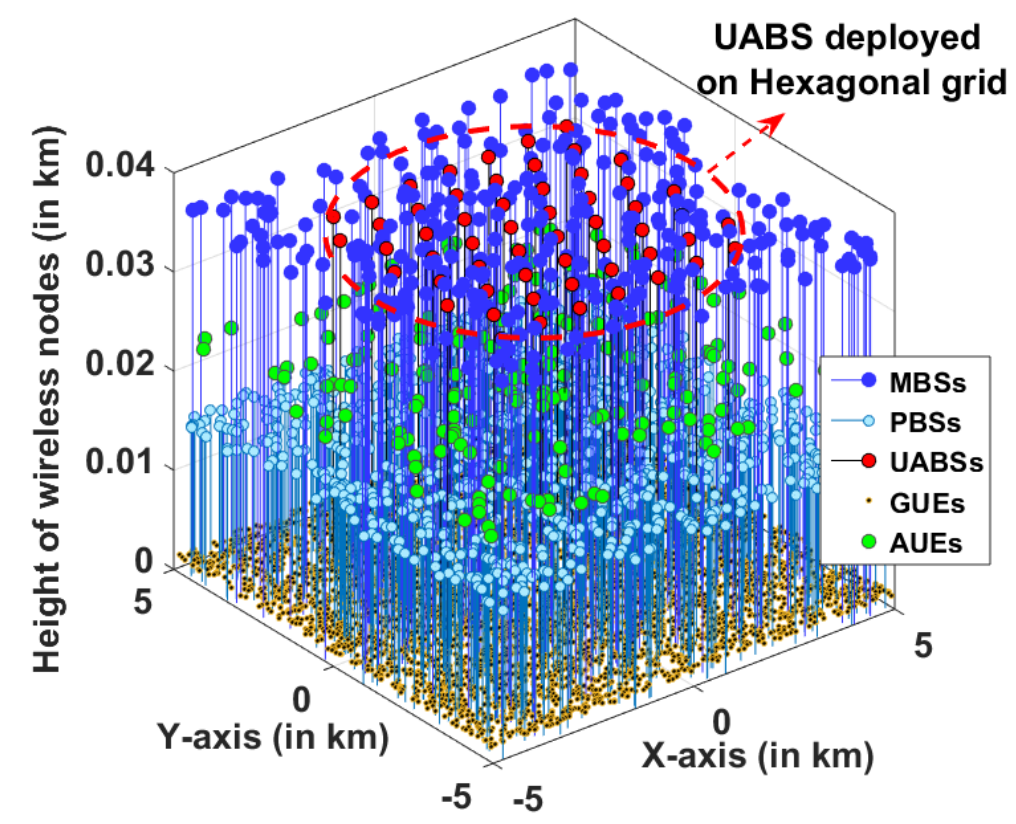

(a) UABSs deployed on a fixed hexagonal grid.

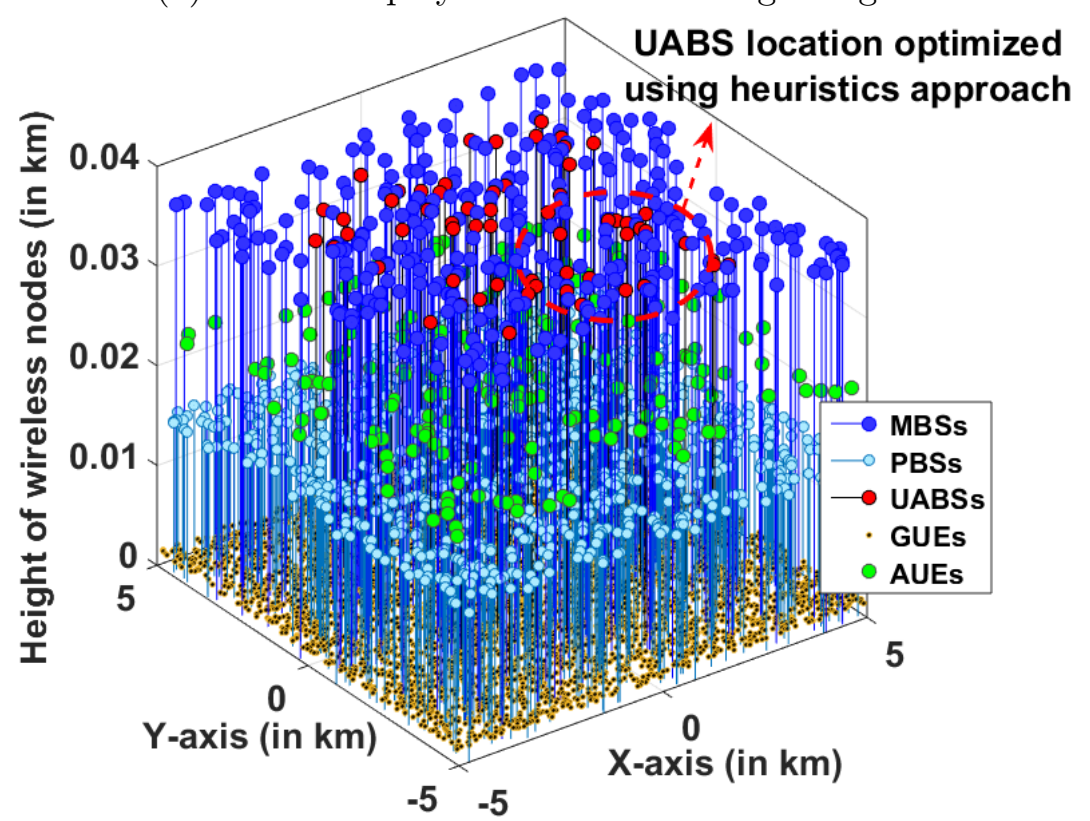

(b) UABS locations optimized using heuristics approach.

Figure 7.4: Three dimensional distribution of ground UEs (GUEs), aerial UEs (AUEs), macro base-stations (MBSs), pico base-stations (PBSs), and unmanned aerial base-stations (UABSs) in three-tier AG-HetNet. The densities and deployment heights each of the wireless nodes are specified in Table 7.2. 


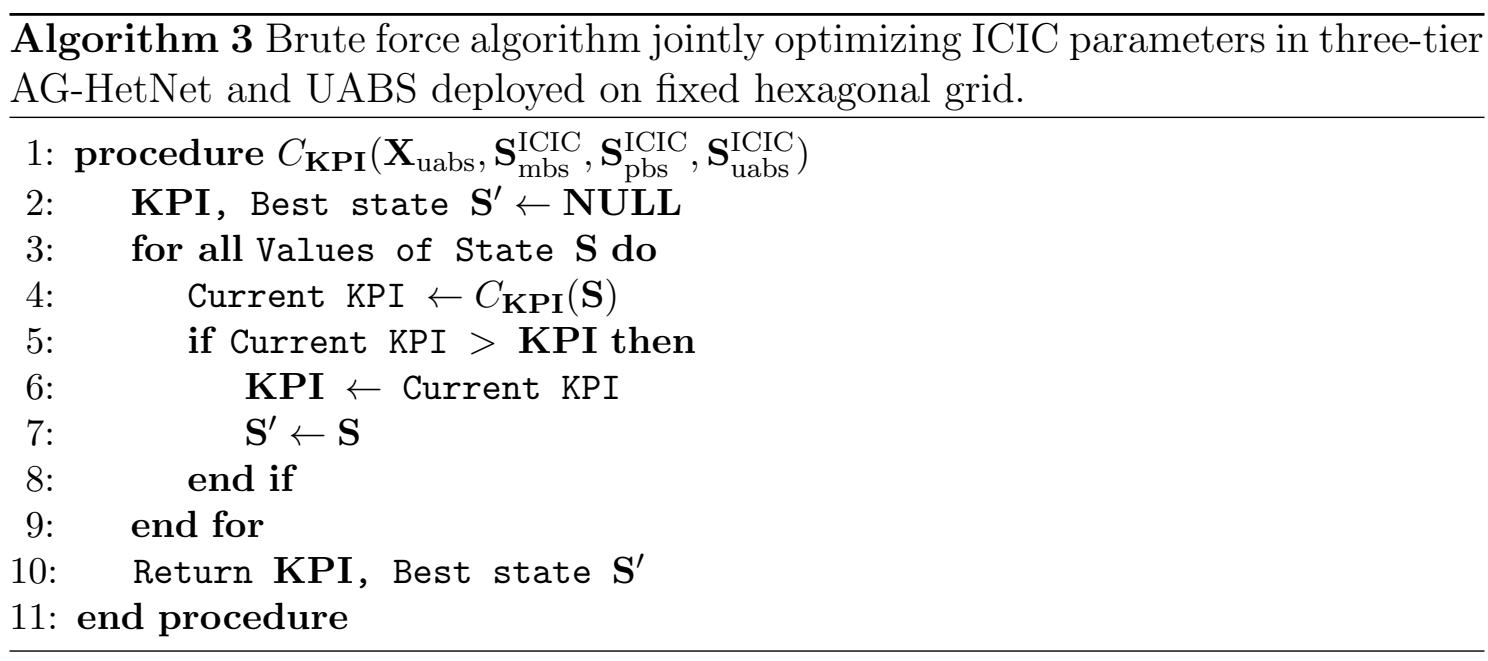

spectively, for each UABS. Using these variable definitions, the initial state of the AG-HetNet can be given as $\mathbf{S}=\left[\mathbf{X}_{\text {uabs }}, \mathbf{S}_{\text {mbs }}^{\text {ICIC }}, \mathbf{S}_{\mathrm{pbs}}^{\mathrm{ICIC}}, \mathbf{S}_{\text {uabs }}^{\mathrm{ICIC}}\right]$. However, to reduce the system complexity and simulation runtime, we apply the same $\mathbf{S}_{\text {mbs }}^{\text {ICIC }}$ parameters across all MBSs, $\mathbf{S}_{\mathrm{pbs}}^{\mathrm{ICIC}}$ across all PBSs, and $\mathbf{S}_{\text {uabs }}^{\mathrm{ICIC}}$ across all UABSs.

Using a brute-force algorithm to search for all possible optimal values in a large search space is computationally infeasible. Therefore the UABSs are initially deployed on a fixed hexagonal grid, as shown in Fig. 7.4(a), and every UABS sends its locations and all the base-stations send the spectral efficiency information of its users (AUE and GUE) to a centralized server. Subsequently, a brute-force technique will be used to optimize the only the ICIC network parameters and evaluate the 5pSE and coverage probability for this fixed AG-HetNet. Then, a centralized server can run any appropriate heuristic algorithm to jointly optimize the UABS locations and ICIC parameters, as illustrated in Fig. 7.4(b). Then, for the proposed AG-HetNet the best state $\left(\mathbf{S}_{\mathbf{K P I}}^{\prime}\right)$ of all the possible states, $\mathbf{S}$ is given as

$$
\mathbf{S}_{\mathbf{K P I}}^{\prime}=\arg \max _{S} C_{\mathbf{K P I}}(\mathbf{S}),
$$




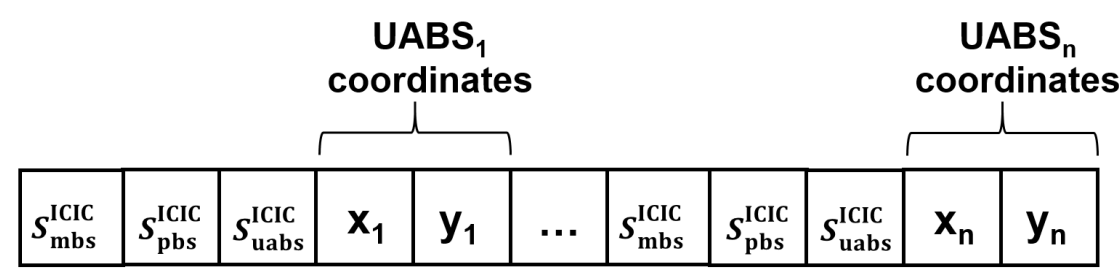

Figure 7.5: An example of a chromosome for ICIC simulation in three-tier AGHetNet, where the UABS locations and ICIC parameters $\mathbf{S}_{\mathrm{mbs}}^{\mathrm{ICIC}}, \mathbf{S}_{\mathrm{pbs}}^{\mathrm{ICIC}}$, and $\mathbf{S}_{\text {uabs }}^{\mathrm{ICIC}}$ are jointly optimized.

where $C_{\mathbf{K P I}}($.$) is an objective function wherein \mathbf{K P I} \in(5 \mathrm{pSE}, \mathrm{COV})$ then $C_{5 \mathrm{pSE}}($. denotes the objective function for $5 \mathrm{pSE}$ and $C_{\mathrm{cov}}($.$) denotes the objective function$ for coverage probability.

Using the brute-force technique described in Algorithm 3 and UABS on a fixed hexagonal grid $\left(\mathbf{X}_{\text {uabs }}\right)$ in a AG-HetNet, the optimal values of the best state $\left(\mathbf{S}^{\prime}{ }_{\mathbf{K P I}}\right)$ of all the possible states $\mathbf{S}$ can be vectorized into $\mathbf{S}_{\mathbf{K P I}}^{\prime}=\left[\mathbf{X}_{\text {uabs }},{\mathbf{\mathbf { S } ^ { \prime }}}_{\mathrm{mbs}}^{\prime \mathrm{ICIC}}, \mathbf{S}_{\mathrm{pbs}}^{\mathbf{I C I C}}, \mathbf{S}_{\text {uabs }}^{\prime \text { ICIC }}\right]$. Whereas, using heuristics algorithm proposed in Algorithm 4 and Algorithm 5, the the optimal values of the best state $\left(\mathbf{S}_{\mathbf{K P I}}^{\prime}\right)$ of all the possible states $\mathbf{S}$ can be vectorized into $\mathbf{S}_{\mathbf{K P I}}^{\prime}=\left[\mathbf{X}_{\text {uabs }}^{\prime}, \mathbf{S}_{\text {mbs }}^{\prime \prime \text { ICIC }}, \mathbf{S}_{\text {pbs }}^{\prime \text { ICIC }}, \mathbf{S}_{\text {uabs }}^{\prime \text { ICIC }}\right]$. Where, $\mathbf{X}_{\text {uabs }}^{\prime}$ is the optimal UABS location and $\mathbf{S}_{\text {mbs }}^{\prime \text { ICIC }}, \mathbf{S}_{\mathrm{pbs}}^{\prime \text { ICIC }}$, and $\mathbf{S}_{\text {uabs }}^{\prime \text { ICIC }}$ are the optimal ICIC values for MBSs, PBSs, and UABSs, respectively.

\subsubsection{Heuristic Algorithms}

With the purpose of improving computational efficiency and obtain the diverse optimal solution, we consider the GA and eHSGA as the heuristic algorithms [35, $36,288]$ to simultaneously optimize UABS locations and ICIC parameters in the large search space.

Genetic algorithm considered in this chapter follows the approach similar to that in $[35,36]$. This technique considers a population of candidate solutions which is 


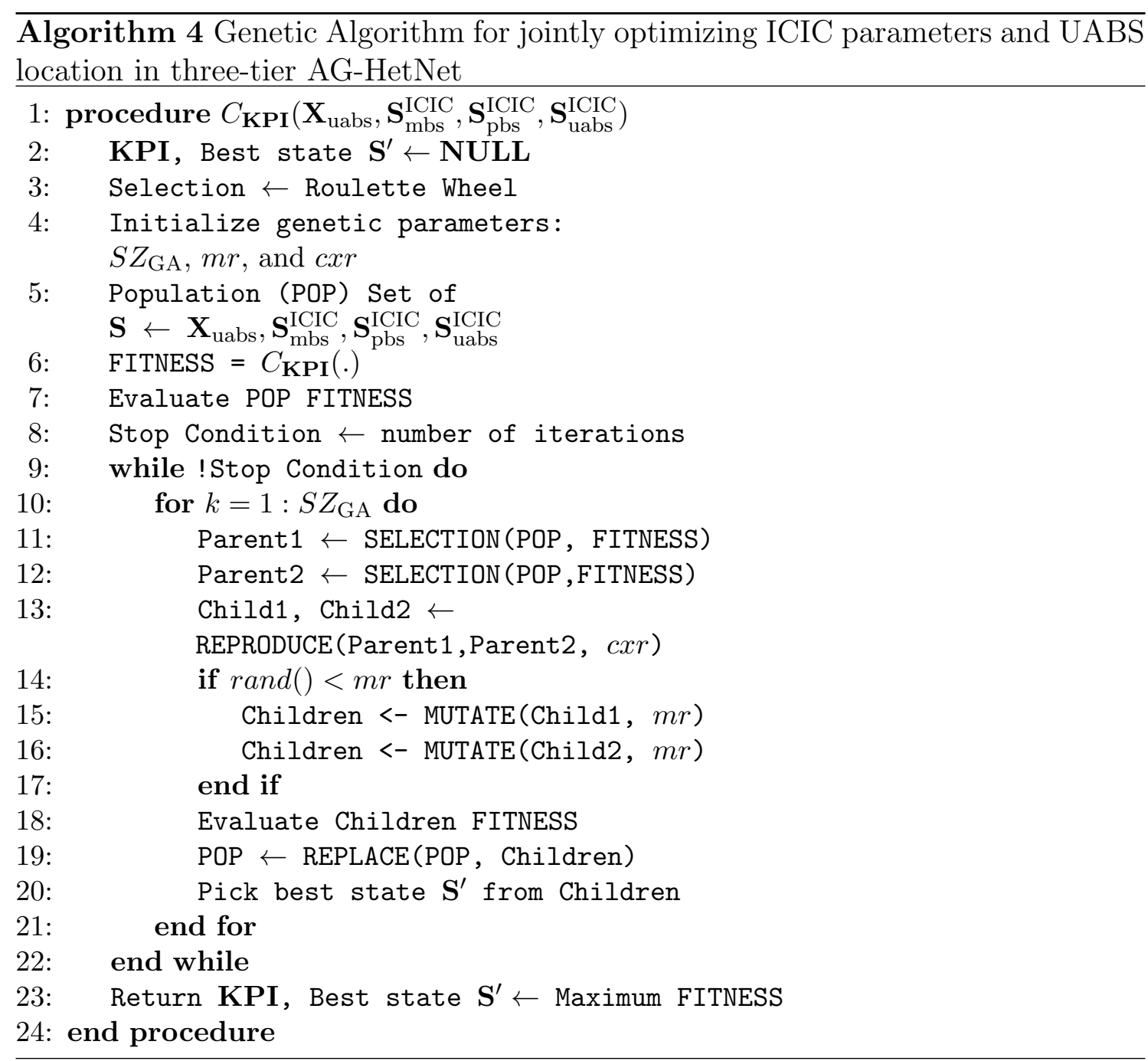

evolved towards an optimal solution or near-optimal solution. Each candidate solution has a set of chromosomes that are evaluated and then altered and mutated to form next-generation offspring [289]. Through an iterative process, adaptive-fit individuals in a population and environment are obtained. In this GA approach, the UABS coordinates $\left(\mathbf{X}_{\text {uabs }}\right)$ and ICIC network parameters $\left(\mathbf{S}_{\mathrm{mbs}}^{\mathrm{ICIC}}, \mathbf{S}_{\mathrm{pbs}}^{\mathrm{ICIC}}, \mathbf{S}_{\text {uabs }}^{\mathrm{ICIC}}\right)$ form the GA population, and a subsequent chromosome is illustrated in Fig. 7.5. With crossover rate of $c x r$ and mutation rate of $m r$ probabilities for a GA population size of $S Z_{\mathrm{GM}}$, the main steps used to optimize the UABS locations and ICIC network parameters while computing the maximum 5pSE and coverage probability is 


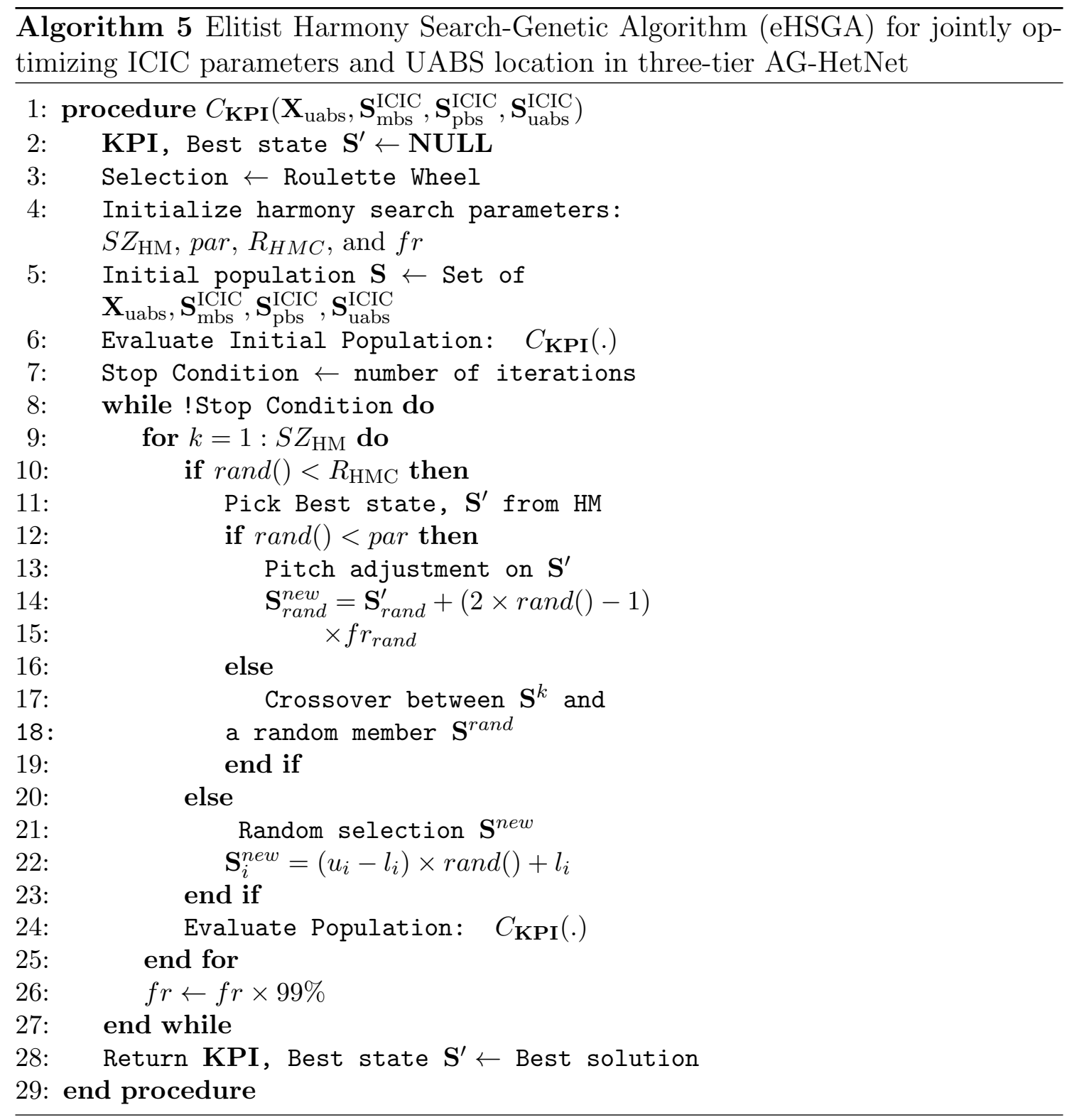

described in Algorithm 4. However, GA has limitations in terms of low convergence speed and requires high computation time.

Further, to obtain possible improvement over GA, we explore elitist harmony search based on the genetic algorithm proposed in [288]. We extend the approach of the proposed hybrid algorithm to optimize the UABS locations and ICIC network parameters. In the main procedure, the initial population generated using GA is 
considered as the harmony memory and the chromosome illustrated in Fig. 7.5 as the harmony. Let $\mathbf{S}_{i}$ be the $i$ th element of harmony $\mathbf{S} ; u_{i}$ and $l_{i}$ are the upper/lower bounds of the $i$ th variable; $\operatorname{rand}()$ is a uniformly-distributed real random number in $[0,1][288]$. Then, we initialize the eHSGA parameters such as the harmony memory size $\left(S Z_{\mathrm{HM}}\right)$, harmony memory consideration rate $\left(R_{\mathrm{HMC}}\right)$, pitch adjustment rate (par), maximum number of improvisation $\left(N_{\mathrm{IMP}}\right)$ and fret width $(f r)$. The $R_{\mathrm{HMC}}$ and par parameters in harmony search are critical to controlling the performance and speed of the convergence of the solution. To guarantee that the hybrid search method can expeditiously detect its way by avoiding local optima and the solution reached is diverse, $R_{\mathrm{HMC}}$ is updated linearly decreasing with the iteration and par is dynamically adapted in linearly increasing rates. Then, we evaluate the fitness of every harmony in the harmony memory and sort the harmony memory in descending order of best fitness. This sorting ensures the harmony memory head always points to the best harmony member. Subsequently, using selection, crossover, and mutation, new harmony memory is generated. A merge rule is applied to sorted harmony memory and new harmony memory to generate an elitist harmony memory. As described in Algorithm 5, through an iterative process, elitism is employed in the search process of obtaining optimal UABS locations and ICIC network parameters.

\subsection{Simulation Results}

In this section, with the help of extensive Matlab-based computer simulation and system parameters set to the values given in Table 7.2 , we conduct a comparative study of the two KPIs of the proposed AG-HetNet, with/without ICIC techniques

for different deployment heights of UABS and while considering brute-force, GA, and eHSGA optimization techniques. In order to reduce the complexity of the opti- 
Table 7.2: System and simulation parameters for three-tier AG-HetNet.

\begin{tabular}{l|l}
\hline Parameters & Value \\
\hline Simulation area $\left(A_{\text {sim }}\right)$ & $100 \mathrm{~km}^{2}$ \\
MBS, PBS, GUE, AUE densities & $4,12,100$, and $1.8 \mathrm{per}^{2}$ \\
Number of UABS & 60 \\
MBS, PBS, and UABS transmit powers & 46,30, and $26 \mathrm{dBm}$ \\
Height of MBS, PBS, and UABS & 36 and $15 \mathrm{~m}$ \\
Height of UABS & 25,36, and $50 \mathrm{~m}$ \\
Height of GUE and AUE & 1.5 and $22.5 \mathrm{~m}$ \\
PSC LTE Band 14 center frequency & $763 \mathrm{MHz}$ for downlink \\
Power reduction factor $\alpha_{\text {mbs }}$ and $\alpha_{\mathrm{pbs}}$ & 0 to 1 \\
USF Duty cycle $\beta_{\text {mbs }}, \beta_{\mathrm{pbs}}$ & 0 to $100 \%$ \\
Scheduling threshold for MUEs $\left(\rho_{\mathrm{mbs}}\right)$ & $20 \mathrm{~dB}$ to $40 \mathrm{~dB}$ \\
Scheduling threshold for PUEs $\left(\rho_{\mathrm{pbs}}\right)$ & $-10 \mathrm{~dB}$ to $10 \mathrm{~dB}$ \\
Scheduling threshold for UUEs $\left(\rho_{\text {uabs }}\right)$ & $-5 \mathrm{~dB}$ to $5 \mathrm{~dB}$ \\
Range expansion bias for $\tau_{\text {uabs }}, \tau_{\text {uabs }}$ & $0 \mathrm{~dB}$ to $12 \mathrm{~dB}$ \\
GA population size $\left(S Z_{\mathrm{GA}}\right)$ and generation number & 60 and 100 \\
GA crossover $(c x r)$ and mutation $(m r)$ probabilities & 0.7 and 0.1 \\
eHSGA population size $\left(S Z_{\mathrm{HM}}\right)$ & 60 \\
Harmonic memory pitch adjustment rate $($ par $)$ & max $=0.8$, min $=0.4$ \\
Harmonic memory consideration rate $\left(R_{\mathrm{HMC}}\right)$ & max $=0.8$, min $=0.2$ \\
Harmonic memory fret $(f r)$ & 1 \\
\hline
\end{tabular}

mization algorithms and simulation runtime, the deployment height of UABS is not considered when optimizing UABS locations, i.e., the UABS locations are optimized in 2D. However, in this chapter, we do investigate and compare the performance of the KPIs by manually deploying UABS at practical heights of $25 \mathrm{~m}, 36 \mathrm{~m}$, and $50 \mathrm{~m}$.

\subsubsection{KPI Optimization using Brute Force Technique}

The 3D surface plot in Fig. 7.6, Fig. 7.7, and Fig. 7.8 illustrates the combined effect of CRE at PBSs and UABSs (along $\mathrm{x}$ - and y-axes) on the coverage probability and 5pSE (along the z-axis) of the wireless network. In an initial inspection of Fig. 7.6, Fig. 7.7 and Fig. 7.8, we can intuitively conclude that FeICIC performs better when 


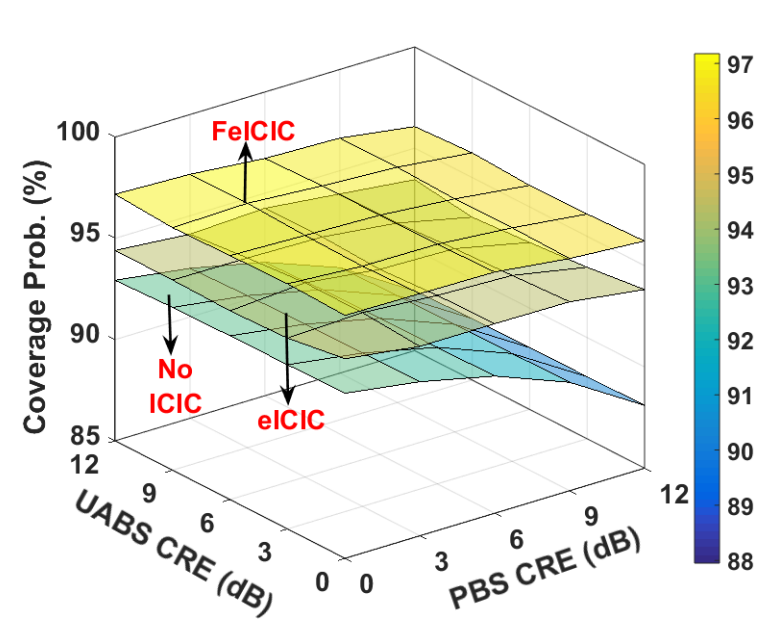

(a) Coverage probability vs. CRE.

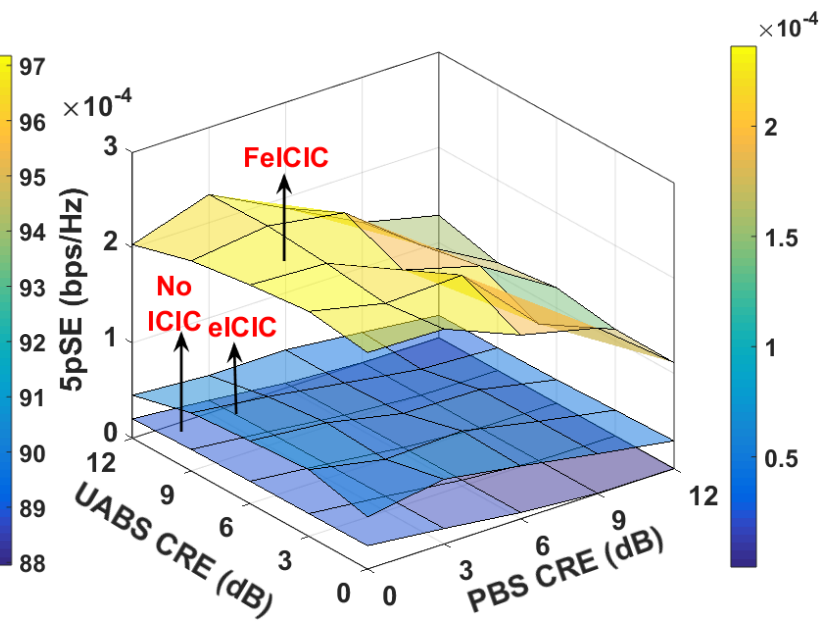

(b) Peak 5pSE vs. CRE.

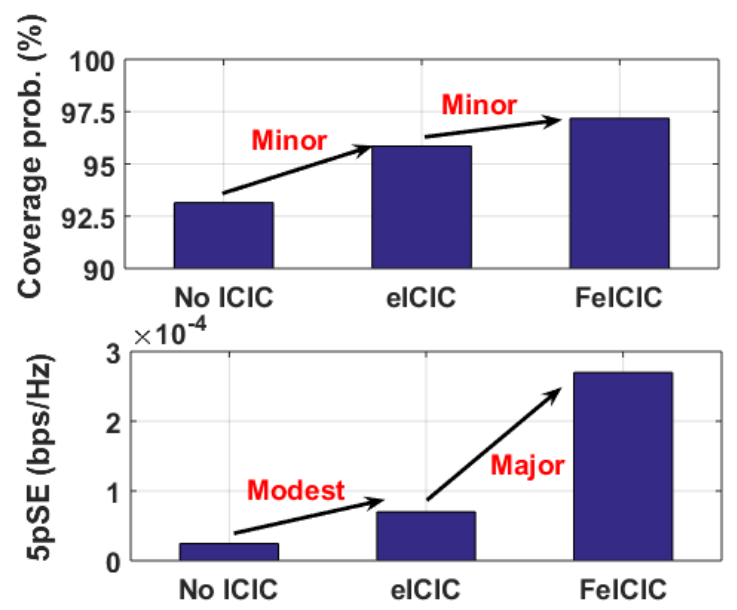

(c) Performance improvement of the two KPIs.

Figure 7.6: The effects of combined CRE at PBS and UABS on the two KPIs of the network, with and without ICIC in three-tier AG-HetNet; when the UABS are deployed at height of $25 \mathrm{~m}$.

compared to eICIC and without any ICIC techniques. The comparative analysis of Fig. 7.6(c), Fig. 7.7(c), and Fig. 7.8(c) reveals that the improvement in coverage probability is less significant, but the $5 \mathrm{pSE}$ improvement is significant.

When UABS is deployed at a height higher than PBS but lower than MBS, i.e., UABS deployment height is $25 \mathrm{~m}$, coverage probability with eICIC sees a minor improvement over the absence of any ICIC, and FeICIC also sees a minor improvement 


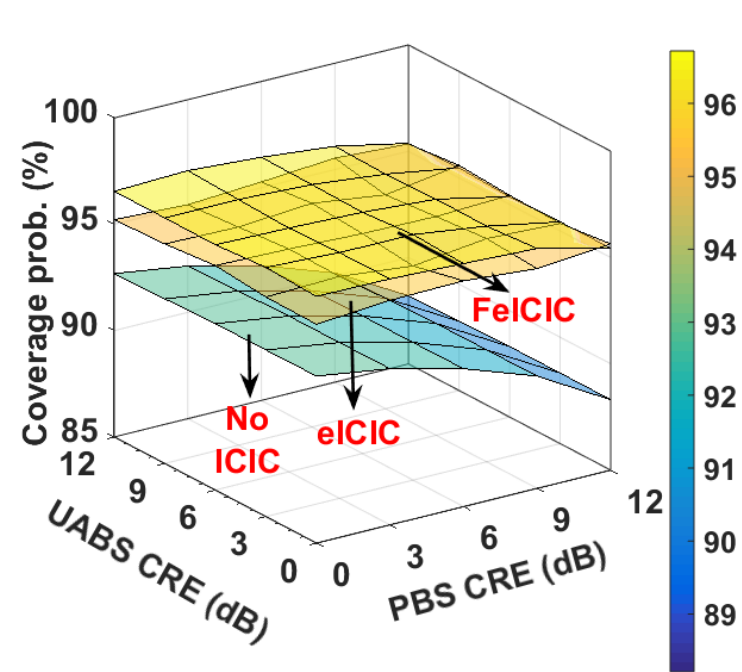

(a) Coverage probability vs. CRE.

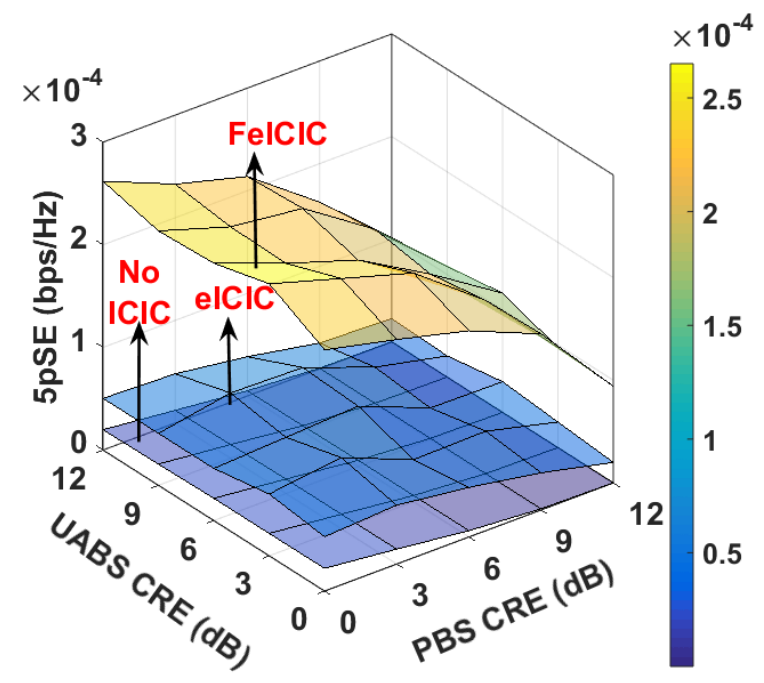

(b) Peak 5pSE vs. CRE.
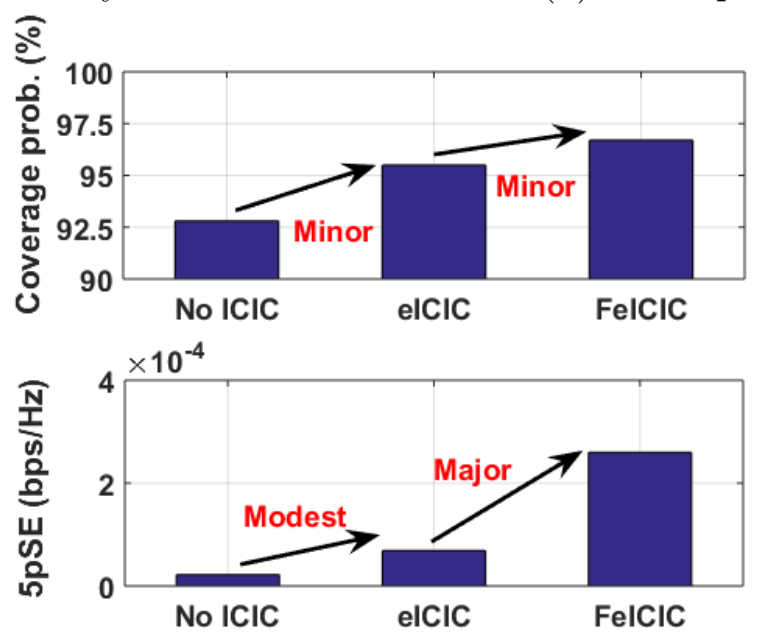

(c) Performance improvement of the two KPIs.

Figure 7.7: The effects of combined CRE at PBS and UABS on the two KPIs of the network, with and without ICIC in three-tier AG-HetNet; when UABS are deployed at height of $36 \mathrm{~m}$.

over eICIC. With increasing CRE of UABS and lower CRE for PBS, the peak values of the coverage probability for the ICIC techniques are observed when the UABS CRE is between $9-12 \mathrm{~dB}$, and PBS CRE varies between $0-3 \mathrm{~dB}$. For 5pSE, eICIC sees modest improvement over the absence of any ICIC, and FeICIC sees a 


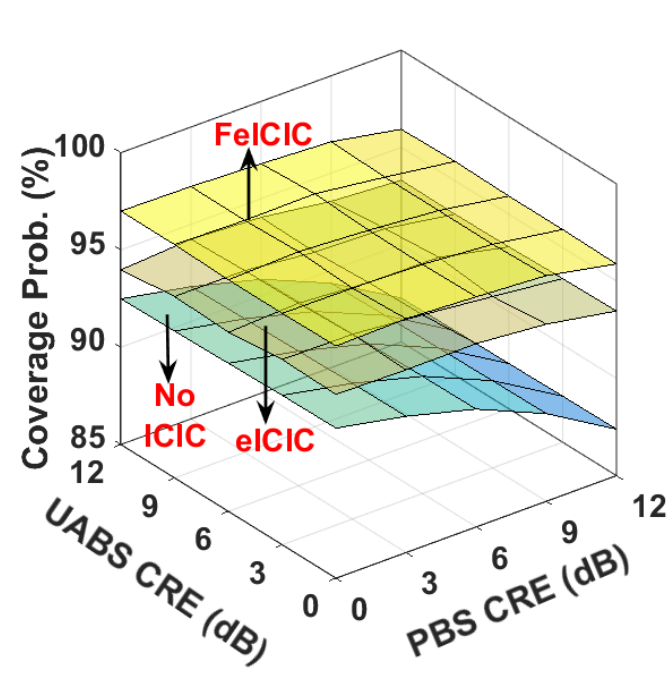

(a) Coverage prob. vs. CRE.

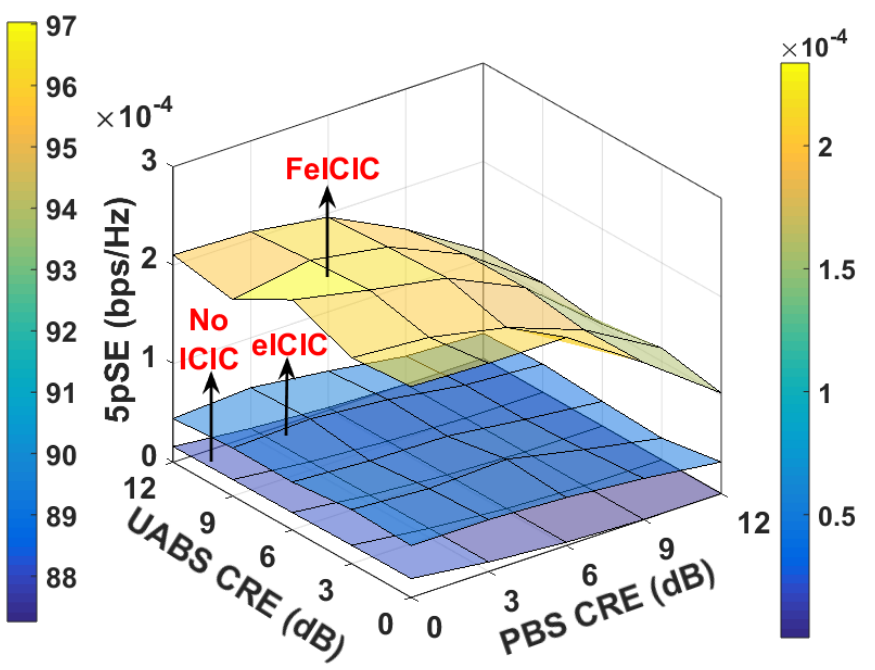

(b) Peak 5pSE vs. CRE.
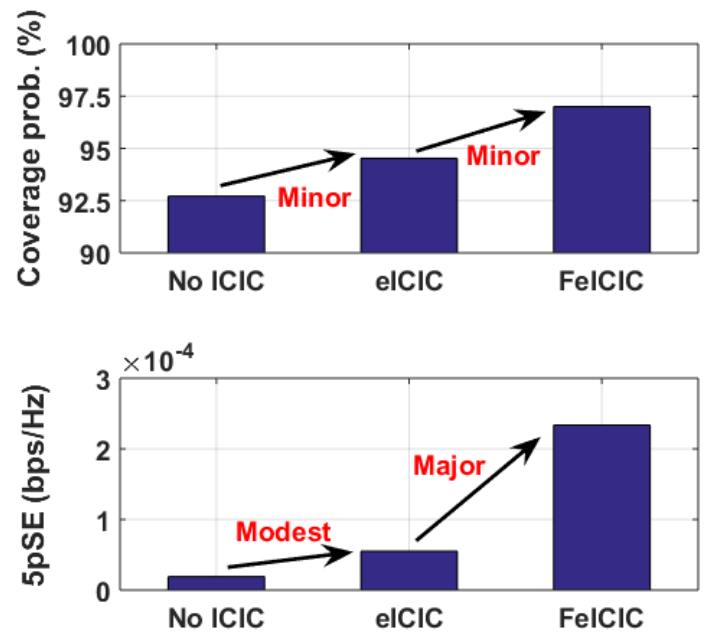

(c) Performance improvements of the two KPIs.

Figure 7.8: The effects of combined CRE at PBS and UABS on the two KPIs of the network, with and without ICIC in three-tier AG-HetNet; when UABS are deployed at height of $50 \mathrm{~m}$.

major improvement over eICIC. The peak values of $5 \mathrm{pSE}$ for the ICIC techniques are observed for lower values of CRE between $3-6 \mathrm{~dB}$ for both UABS and PBS.

Whereas, when UABS are deployed at the same height as MBS, i.e., UABS deployment height is $36 \mathrm{~m}$, coverage probability with eICIC sees a minor improvement over the absence of any ICIC, and FeICIC also sees a minor improvement over eICIC. 
The peak values of coverage probability for the ICIC techniques are observed for lower values of CRE between $3-6 \mathrm{~dB}$ for both UABS and PBS. For 5pSE, eICIC sees modest improvement over the absence of any ICIC, and FeICIC sees a major improvement of over eICIC. With increasing CRE of UABS and lower CRE for PBS, the peak values of the $5 \mathrm{pSE}$ for the ICIC techniques are observed when the UABS CRE is between $9-12 \mathrm{~dB}$, and PBS CRE varies between $0-3 \mathrm{~dB}$.

Finally, when the UABS is deployed at a height higher than MBS, i.e., UABS deployment height is $50 \mathrm{~m}$, coverage probability with eICIC sees a minor improvement over the absence of any ICIC, and FeICIC also sees a minor improvement over eICIC. With increasing CRE of UABS and lower CRE for PBS, the peak values of the coverage probability for the ICIC techniques are observed when the UABS CRE is between $9-12 \mathrm{~dB}$, and PBS CRE varies between $0-3 \mathrm{~dB}$. For $5 \mathrm{pSE}$, eICIC

sees modest improvement over the absence of any ICIC, and FeICIC sees a major improvement of over eICIC. With increasing CRE of UABS and lower CRE for PBS, the peak values of the $5 \mathrm{pSE}$ for the ICIC techniques are observed when the UABS CRE is $9 \mathrm{~dB}$, and PBS CRE varies between $0-3 \mathrm{~dB}$.

Overall, when UABSs are deployed on a fixed hexagonal grid and using the brute-force technique to optimize ICIC parameters, the peak values of 5pSE and coverage probability is observed when UABS is deployed at the low altitude of $25 \mathrm{~m}$ and using Release-11 reduced power FeICIC technique as seen in Fig. 7.6, Fig. 7.7, and Fig. 7.8 .

\subsubsection{KPI Optimization using Genetic Algorithm}

In the following, we discuss the key observations when UABS locations and ICIC network parameters are jointly optimized through GA. In Fig. 7.9 we plot the peak 
coverage probability and 5pSE values (along the z-axis) against the combined effect of CRE at PBSs and UABSs (along $\mathrm{x}$ - and y-axes) of the wireless network, when UABS are deployed at the height of $25 \mathrm{~m}, 36 \mathrm{~m}$, and $50 \mathrm{~m}$ and while considering GA. In an initial inspection of Fig. 7.9(a), Fig. 7.9(b), and Fig. 7.9(c), we can intuitively conclude that FeICIC performs better when compared to eICIC and without any ICIC techniques.

We present the comparative analysis of the peak value observations of the two KPIs in Fig. 7.9(d), Fig. 7.9(e), and Fig. 7.9(f). When UABS is deployed at a height higher than PBS but lower than MBS, i.e., UABS deployment height is 25 $\mathrm{m}$, coverage probability with eICIC sees modest improvement in the absence of any ICIC, and FeICIC sees a minor improvement over eICIC. The peak values of coverage probability for the ICIC techniques are observed for CRE values between $3-6 \mathrm{~dB}$ for UABS, and PBS CRE varies between $0-12 \mathrm{~dB}$. For 5pSE, eICIC sees a major improvement in the absence of any ICIC, and FeICIC sees a modest improvement over eICIC. The peak values of 5pSE for the ICIC techniques are observed for moderate values of CRE between $3-6 \mathrm{~dB}$ for UABS and is $0-3 \mathrm{~dB}$ for PBS.

Whereas, when UABS are deployed at the same height as MBS, i.e., UABS deployment height is $36 \mathrm{~m}$, coverage probability with eICIC sees modest improvement in the absence of any ICIC, and FeICIC sees a minor improvement over eICIC. The peak values of coverage probability for the ICIC techniques are observed for larger values of CRE between $6-12 \mathrm{~dB}$ for UABS, and PBS CRE varies between $0-12$ dB. For 5pSE, eICIC sees a major improvement over the absence of any ICIC, and FeICIC sees a minor improvement over eICIC. The peak values of 5pSE for the ICIC techniques are observed for moderate values of CRE between $3-6 \mathrm{~dB}$ for UABS and is $0-3 \mathrm{~dB}$ for PBS. 

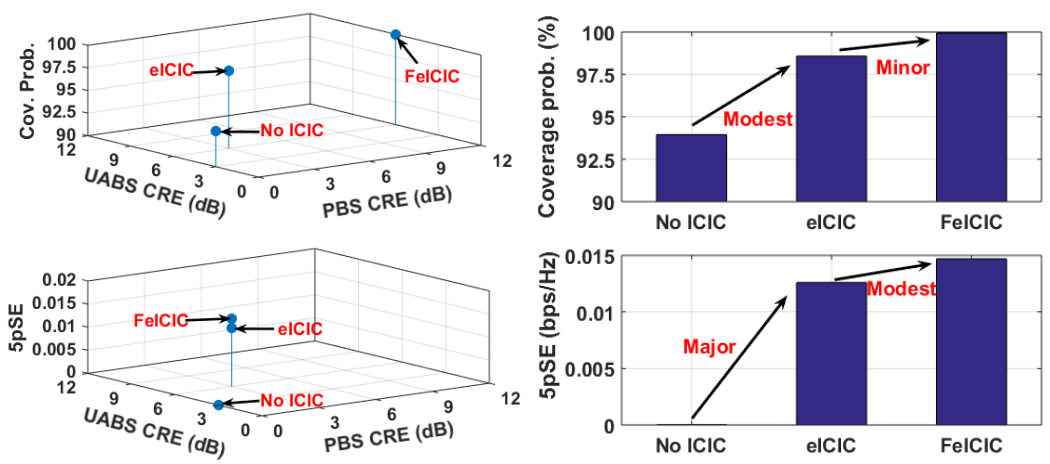

(a) Peak value observation (b) Performance improvement when UABS are deployed at the when UABS are deployed at the height of $25 \mathrm{~m}$.

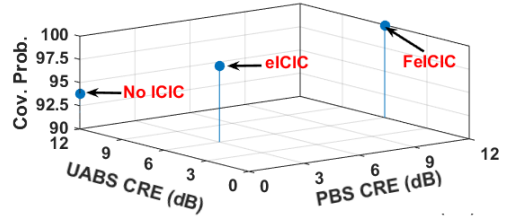
height of $25 \mathrm{~m}$.
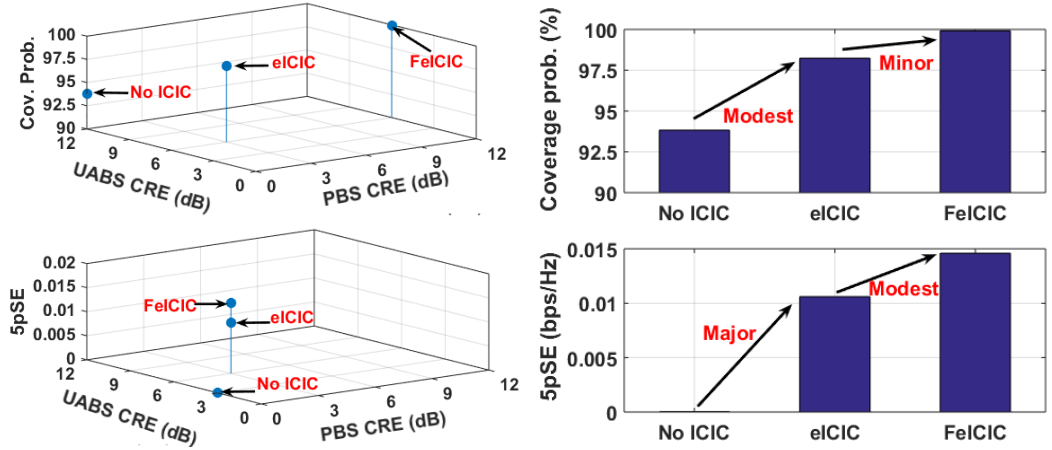

(c) Peak value observation (d) Performance improvement when UABS are deployed at the when UABS are deployed at the height of $36 \mathrm{~m}$. height of $36 \mathrm{~m}$.
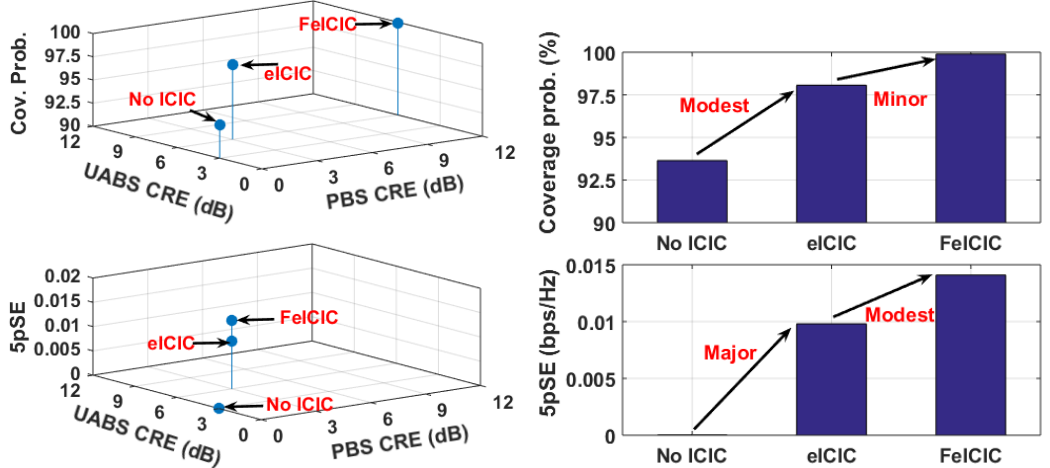

(e) Peak value observation (f) Performance improvements when UABS are deployed at the when UABS are deployed at the height of $50 \mathrm{~m}$. height of $50 \mathrm{~m}$.

Figure 7.9: A combined effect of CRE at PBS and UABS on the two KPIs of the network, with and without ICIC in three-tier AG-HetNet. When UABS are deployed at the height of $25 \mathrm{~m}, 36 \mathrm{~m}$, and $50 \mathrm{~m}$ and while considering GA. 
Finally, when the UABS is deployed at a height higher than MBS, i.e., UABS deployment height is $50 \mathrm{~m}$, coverage probability with eICIC sees modest improvement over the absence of any ICIC, and FeICIC sees a minor improvement over eICIC. The peak values of coverage probability for the ICIC techniques are observed for moderate values of CRE between 3-6 dB for UABS, and PBS CRE varies between 0-12 dB. For 5pSE, eICIC sees a major improvement over the absence of any ICIC, and FeICIC sees a minor improvement over eICIC. The peak values of 5pSE for the ICIC techniques are observed for moderate values of CRE between $3-6 \mathrm{~dB}$ for UABS and is $0-3 \mathrm{~dB}$ for PBS.

Overall, using GA to joint optimize the UABS locations and ICIC parameters, the peak values of $5 \mathrm{pSE}$ and coverage probability are observed when UABS is deployed at a low altitude of $25 \mathrm{~m}$ and using Release-11 reduced power FeICIC technique as seen in Fig. 7.9.

\subsubsection{KPI optimization using eHSGA}

In the following, we discuss the key observations when UABS locations and ICIC network parameters are jointly optimized through the eHSGA algorithm. In Fig. 7.10 we plot the peak coverage probability and $5 \mathrm{pSE}$ values (along the z-axis) against the combined effect of CRE at PBSs and UABSs (along x- and y-axes) of the wireless network, when UABS are deployed at the height of $25 \mathrm{~m}, 36 \mathrm{~m}$, and $50 \mathrm{~m}$. In an initial inspection of Fig. 7.10(a), Fig. 7.10(b), and Fig. 7.10(c), we can intuitively conclude that FeICIC performs better when compared to eICIC and without any ICIC techniques.

Whereas, in Fig. 7.10(d), Fig. 7.10(e), and Fig. 7.10(f) we compare the peak value of the two KPIs. When UABS is deployed at a height higher than PBS but 
lower than MBS, i.e., UABS deployment height is $25 \mathrm{~m}$, coverage probability with eICIC sees modest improvement over the absence of any ICIC, and FeICIC sees a minor improvement over eICIC. The peak values of coverage probability for the ICIC techniques are observed for higher values of CRE between $9-12 \mathrm{~dB}$ for UABS and moderate CRE values between 3-9 dB for PBS. For 5pSE, eICIC sees a major improvement over the absence of any ICIC, and FeICIC sees a modest improvement over eICIC. The peak values of 5pSE for the ICIC techniques observed for the CRE values between $3-12 \mathrm{~dB}$ for UABS and lower CRE values between $3-6 \mathrm{~dB}$ for PBS.

Whereas, when UABS are deployed at the same height as MBS, i.e., UABS deployment height is $36 \mathrm{~m}$, coverage probability with eICIC sees modest improvement in the absence of any ICIC, and FeICIC sees a minor improvement over eICIC. The peak values of coverage probability for the ICIC techniques are observed for higher values of CRE between $9-12 \mathrm{~dB}$ for UABS and moderate CRE values between $3-6 \mathrm{~dB}$ for PBS. For 5pSE, eICIC sees a major improvement over the absence of any ICIC, and FeICIC sees a minor improvement over eICIC. The peak values of $5 \mathrm{pSE}$ for the ICIC techniques are observed for CRE values between $0-9 \mathrm{~dB}$ for UABS and is $3 \mathrm{~dB}$ for PBS.

Finally, when the UABS is deployed at a height higher than MBS, i.e., UABS deployment height is $50 \mathrm{~m}$, coverage probability with eICIC sees modest improvement in the absence of any ICIC, and FeICIC sees a minor improvement over eICIC. The peak values of coverage probability for the ICIC techniques are observed for higher values of CRE between $9-12 \mathrm{~dB}$ for UABS and moderate CRE values between $3-6 \mathrm{~dB}$ for PBS. For 5pSE, eICIC sees a major improvement in the absence of any ICIC, and FeICIC sees a minor improvement over eICIC. The peak values of 5pSE 

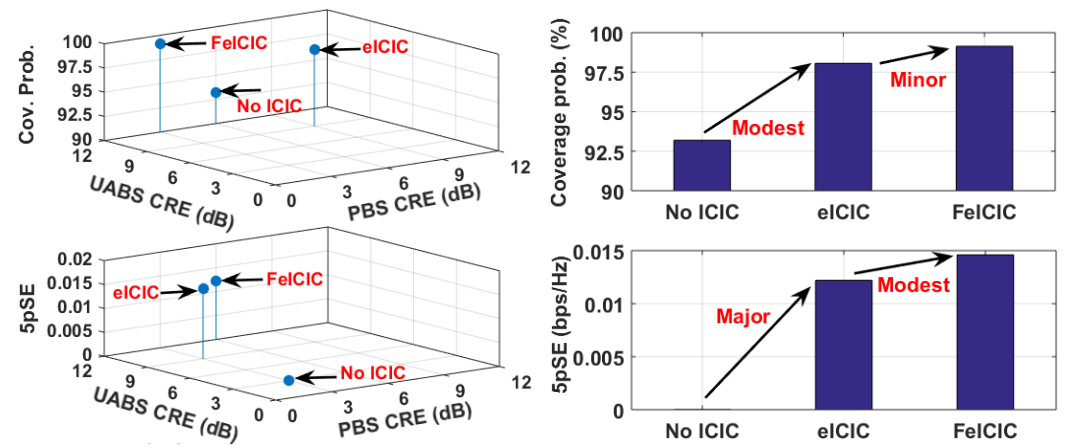

(a) Peak value observation (b) Performance improvement when UABS are deployed at the when UABS are deployed at the height of $25 \mathrm{~m}$.

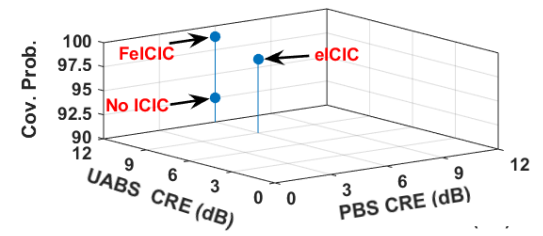
height of $25 \mathrm{~m}$.
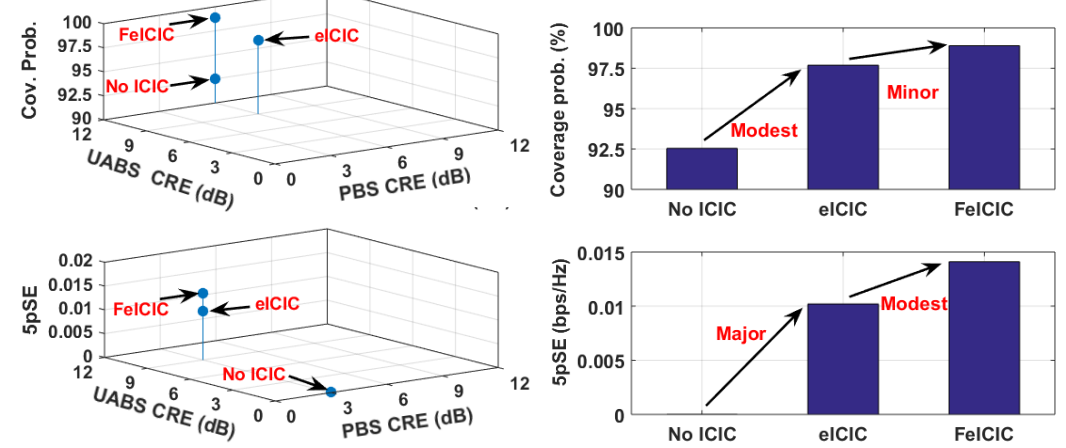

(c) Peak value observation (d) Performance improvement when UABS are deployed at the when UABS are deployed at the height of $36 \mathrm{~m}$.
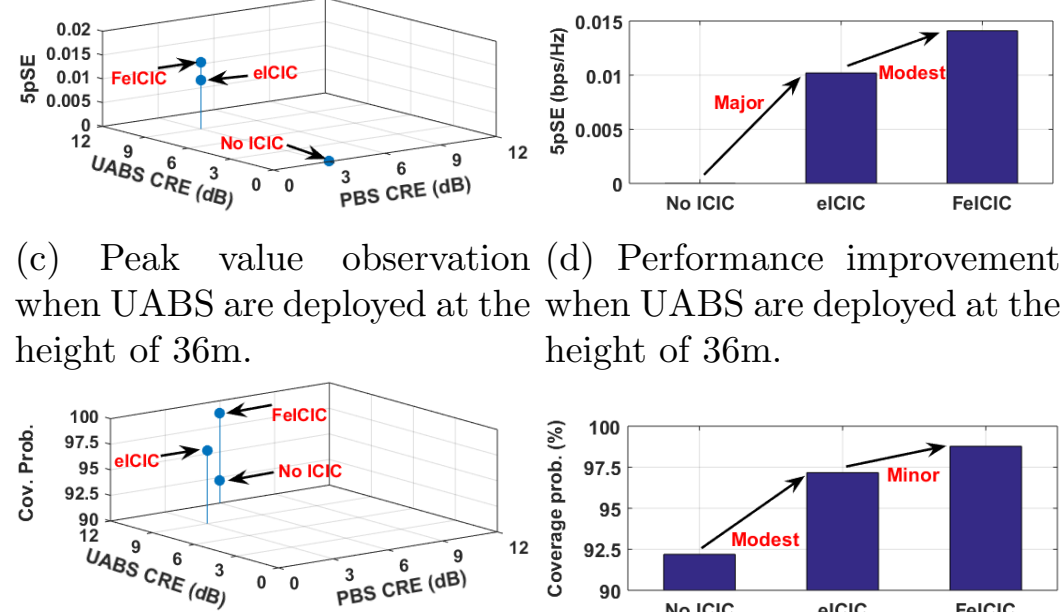
height of $36 \mathrm{~m}$.
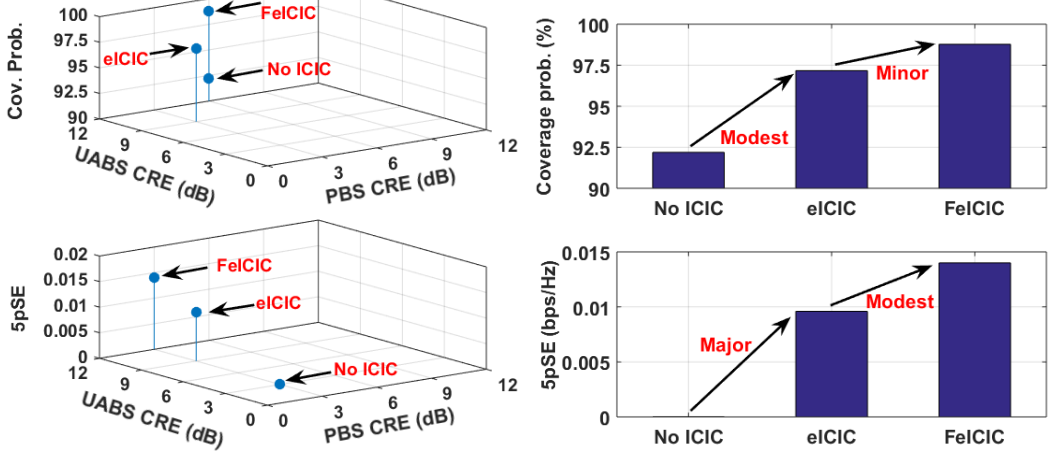

(e) Peak value observation (f) Performance improvements when UABS are deployed at the when UABS are deployed at the height of $50 \mathrm{~m}$.

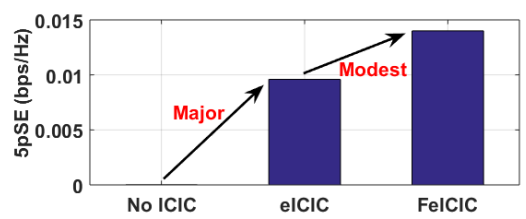
height of $50 \mathrm{~m}$.

Figure 7.10: A combined effect of CRE at PBS and UABS on the two KPIs of the network, with and without ICIC in three-tier AG-HetNet. When UABS are deployed at the height of $25 \mathrm{~m}, 36 \mathrm{~m}$, and $50 \mathrm{~m}$ and while considering eHSGA. 
for the ICIC techniques are observed for CRE values between $3-12 \mathrm{~dB}$ for UABS and is $3 \mathrm{~dB}$ for PBS.

Overall, using eHSGA to joint optimize the UABS location and ICIC parameters, the peak values of 5pSE and coverage probability are higher when UABS is deployed at a low altitude of $25 \mathrm{~m}$ and using Release-11 reduced power FeICIC technique as seen in Fig. 7.10.

\subsubsection{Performance Comparison of the Algorithms}

We summarize our key results from earlier simulations and compare the computation time when using brute-force, GA, and eHSGA techniques with/without ICIC optimization.

\section{Comparison of KPIs}

From the simulation results given in Fig. 7.6, Fig. 7.7, Fig. 7.8, Fig. 7.9, and Fig. 7.10, we observe reduced power FeICIC in Release-11 is seen to outperform Release-10 eICIC and without ICIC in terms of the overall 5pSE and coverage probability of the AG-HetNet. Further inspection reveals that the heuristic techniques (GA and eHSGA) outperform the brute-force technique and observe significant improvement in terms of overall 5pSE and coverage probability of the AG-HetNet. In particular, GA meta-heuristic technique achieved a marginal 5pSE and coverage probability gains of upto $3 \%$ over the hybrid eHSGA optimization technique.

We also observe that the performance of the wireless network is optimal when UABSs are deployed at a height of $25 \mathrm{~m}$. However, as the UABS deployment height increases to $36 \mathrm{~m}$ and $50 \mathrm{~m}$, a gradual decrease in the 5pSE and coverage probability of the wireless network is observed. The higher deployment heights of the UABS 
Table 7.3: Coverage probability peak value observations in $\%$.

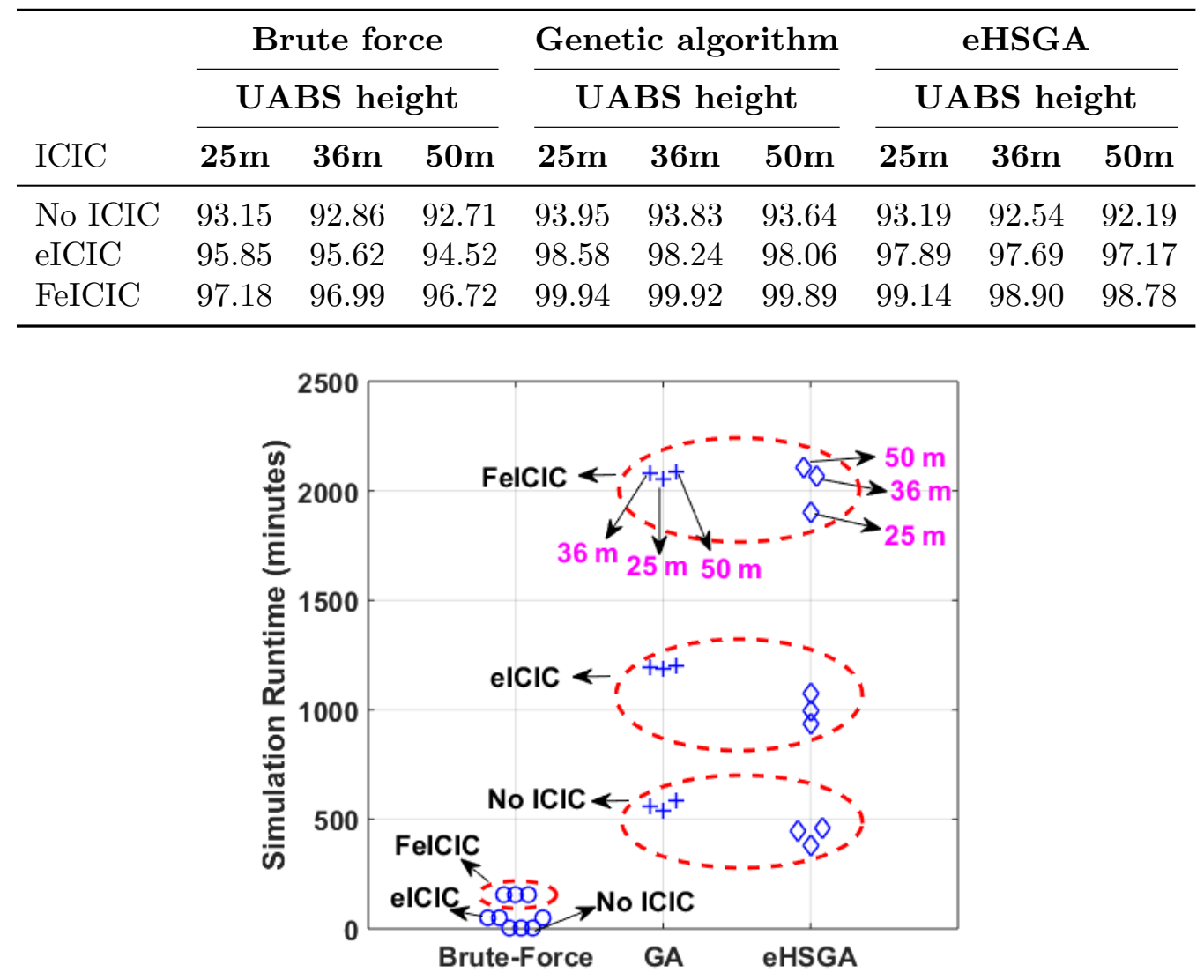

Figure 7.11: Simulation runtime for evaluating a single KPI with/without ICIC techniques in three-tier AG-HetNet; when UABSs are deployed at different heights and using different optimization techniques.

improve LOS to the UEs but also increases interference probability with UEs in cell-edge/CRE, thus degrading the overall performance of the AG-HetNet.

We summarize the peak values observed for coverage probability and 5pSE for with/without ICIC techniques for different deployment heights of UABS; while using brute force, genetic algorithm, and elitist hybridization between harmonic search and genetic algorithm in Table 7.4 and Table 7.3. 


\section{Comparison of Computational Complexity Gains}

In this subsection, we compare the computational complexity gains for brute-force, GA, and eHSGA techniques with/without ICIC optimization and when UABSs are deployed at $25 \mathrm{~m}, 36 \mathrm{~m}$, and $50 \mathrm{~m}$. Using the NCSU high-performance computing server, and Monte-Carlo experimental approach, we calculate the mean runtime for Matlab simulation while evaluating an individual KPI. In Fig. 7.11, we plot the mean runtime required for calculating the peak KPI value with optimal ICIC network parameters and UABS locations using (7.12), brute-force given in Algorithm 3, GA given in Algorithm 4, eHSGA given in Algorithm 5, and the simulation values defined in Table 7.2.

In an initial inspection of Fig. 7.11, we observe reduced power FeICIC technique requires significantly higher computational time when compared to the ABS eICIC and without ICIC techniques, for different deployment heights of UABS (25 m, 36 $\mathrm{m}$, and $50 \mathrm{~m}$ ) and optimization techniques (brute-force, GA, and eHSGA). The reduced power FeICIC technique requires significantly higher computational time because the optimization of power reduction parameters $\left(\alpha_{\mathrm{mbs}}\right.$ and $\left.\alpha_{\mathrm{pbs}}\right)$ of the three-tier LTE subframes, increases the scope of search space when compare to ABS eICIC and without ICIC techniques. Further analysis of Fig. 7.11 reveals that optimization (ICIC parameters and UABS locations) using GA and eHSGA both require significantly more computational time when compared to the brute-force technique. In particular, for lower complexity eICIC and without ICIC techniques, eHSGA observes substantial computational complexity gains between 10.65-29.14\% over GA. Whereas with higher complexity reduced power FeICIC technique, eHSGA observes marginal computational complexity gains of upto 7\% over GA.

Whereas, the UABS deployment height of $50 \mathrm{~m}$ observes a sparse increment in the computation time when compared to $36 \mathrm{~m}$ and $25 \mathrm{~m}$, while jointly optimizing 
the ICIC parameters and UABS locations in the same search space. The higher deployment heights of the UABS improve LOS to the UEs but also increases interference probability with UEs in cell-edge/CRE and consequently increases the computation time to optimize the ICIC parameters and UABS locations.

To summarize, although the computational complexity required to optimize the ICIC network parameters and UAV locations using heuristic techniques (GA and eHSGA) is higher, but is effective in achieving broadband rates. In particular, the heuristic techniques can meet the public safety network requirement of $95 \%$ geographical coverage with broadband rates [59]. Furthermore, from Fig. 7.11, Table 7.3, and Table 7.4, we observe hybrid eHSGA achieves marginal computational complexity gain over meta-heuristic GA technique. Whereas, optimization using GA marginally improves KPI gains when compared to optimization using eHSGA. Hence the determination of an appropriate heuristic algorithm, which achieves the trade-off between computational complexity and finding optimum or close to the optimum solution of a search problem in the real world, requires further investigation. 


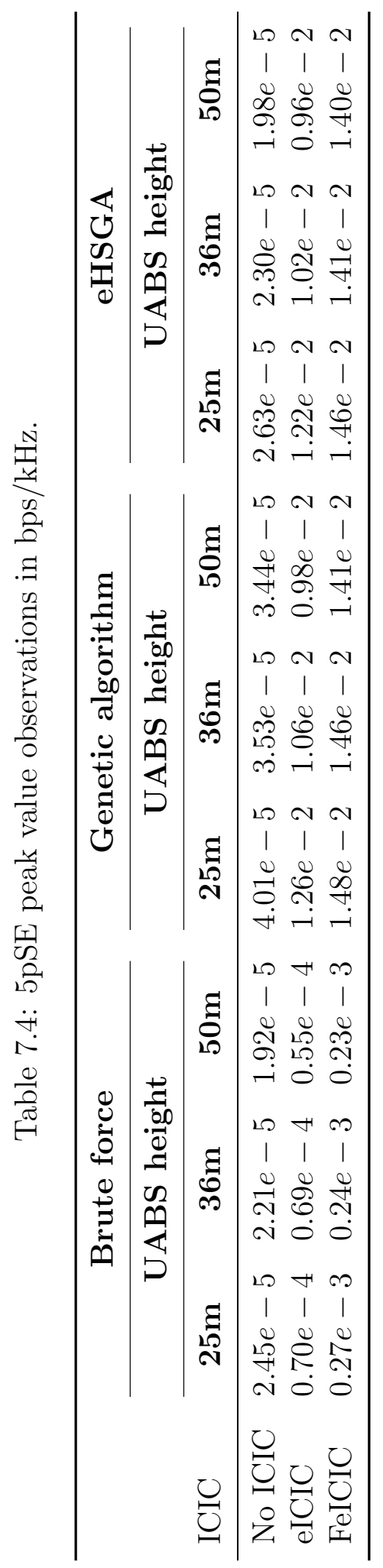




\section{CHAPTER 8}

\section{CONCLUDING REMARKS AND FUTURE WORKS}

Ensuring ubiquitous mission-critical public safety communications to all the first responders in the public safety network is crucial at an emergency site. LTE/LTEAdvanced has emerged as a broadband communication technology that has a potential to transform the capabilities of public safety technologies by providing broadband, ubiquitous, and mission-critical voice and data support. In this research, we studied the legacy and LTE-Advanced public safety networks and addressed some of the important challenges which can be categorized into capacity and coverage enhancements, interference coordination, location optimization of UABSs, and energy efficiency enhancements. In this chapter, we present the concluding remarks on different research tasks explained earlier.

In Chapter 3, an overview of legacy and emerging public safety communication technologies is presented along with the spectrum allocation for public safety usage across all the frequency bands in the United States. As a case study discuss the architecture of FirstNet which is a nationwide coast-to-coast public safety network based of LTE broadband technology. Furthermore, the challenges involved with PSN operations are described, and the benefits of LTE-based PSN over LMRS are also discussed. The comparative survey of legacy and the LTE-based public safety networks, concludes that in the upcoming years, LMRS is bound to stay as the primary PSC solution for mission-critical voice connectivity, alongside LTE providing the much need mission-critical real-time data. The technological advancement achieved through LTE, LTE-Advanced, and $5 \mathrm{G}$ will continue to enhance and transform the PSC capabilities in the future.

In Chapter 4, a simulation study of LMRS and LTE band class 14 technologies is provided using the NS-3 open source tool. From the comparison of the simulation 
results summarized in Table 4.2, it can be deduced that LMRS public safety devices experience larger cell coverage when compared to the band class 14 LTE devices. This volume of cell coverage is critical during the emergency scenario and is necessary to cover the maximum amount emergency prone area. Another rising factor in mission-critical PSC is real-time video communications, high data rate is needed to support real-time multimedia applications. However, the aggregated throughput of LMRS infrastructure is less when compared to the aggregated throughput of LTE band class 14 infrastructure. These data rates experienced by the LTE UEs can definitely support the much needed real-time video communications in several of the PSC scenarios. An adaptation of successful edition of mission-critical PTT over LTE would take some time. In the meantime, applying the individual strength of LMRS and LTE into a converged public safety device to mission-critical voice and much needed mission-critical real-time data support can be beneficial to PSC.

In Chapter 5, an experimental study of capturing APCO-25 and LTE band class 14 signals is conducted using software-defined radio. The capturing of LTE band class 14 signals can assist PSC researchers in detecting coverage holes in PSN, gain knowledge on the quality of service experienced by the UEs and enhance the ability to design better PSN.

In Chapter 6, we show that the mission-critical communications could be maintained and restored by deploying UABSs in the event of any damage to the public safety infrastructure. Through simulations, we compare and analyze the 5pSE of the network for different path-loss models and different UABS deployment strategies. With SPLM, our analysis shows that deployment of the UABSs on a hexagonal grid is close to optimal when the observed interference is limited. In the presence of substantial interference, the GA approach is more effective for deploying UABSs. On the other hand, with OHPLM, the network observes high path-loss when com- 
pared to SPLM. To subdue the effects of high path-loss, the GA approach is shown to be more effective. Our simulation shows that optimizing UABSs locations and ICIC parameters using GA yields significant improvement when compared to the deployment of the UABSs on a hexagonal grid. Finally, we observe that the HetNets with reduced power subframes (FeICIC) yield better 5pSE than that with almost blank subframes (eICIC). In a simulated network with SPLM and when 60 UABS locations are optimized using the GA, the FeICIC observes a modest improvement over eICIC: approximately $17 \%$ and $15 \%$ when $50 \%$ and $97.5 \%$ of the MBSs are destroyed, respectively. On the other hand, with OHPLM and when 60 UABS locations are optimized using the GA, the FeICIC yields a significant improvement over eICIC: approximately $66 \%$ and $51 \%$ when $50 \%$ and $97.5 \%$ of the MBSs are destroyed, respectively. GA is a suitable meta-heuristic technique that relies on bio-inspired approach for finding optimum or close to optimum solution of a search problem. However, the computational complexity required to optimize the considered UAV deployment optimization problems in real world using the GA techniques require further investigations.

In Chapter 7, we provide system-level insights into the LTE-Advanced AGHetNet and evaluate the network performance in terms of coverage probability and 5pSE. In particular, we integrate low-altitude unmanned vehicles as both AUE and UABS into an existing terrestrial network. While considering key design parameters such as the base-station heights, antenna 3DBF, path loss model specific to UE camping, interference coordination, and altitude variation of unmanned vehicles. Using these design considerations and through Monte-Carlo simulations, we maximized the coverage probability and 5pSE of the overall network, while mitigating intercell interference and optimizing ICIC parameters and UABS locations using brute-force and heuristics approach. Finally, our analysis shows that the 
AG-HetNet with reduced power subframes (FeICIC) yields better coverage probability and 5pSE than that with almost blank subframes (eICIC) and without any ICIC. Our simulations results show that the heuristic algorithms (GA and eHSGA) outperform the brute-force technique and achieve effective peak values of coverage probability and 5pSE. In particular, optimization of higher complexity FeICIC using the GA technique achieves marginally better peak KPI values but requires slightly more computational time when compared to hybrid eHSGA. Although the trade-off exists between KPI gains and computational complex gains, simulation results show that hybrid eHSGA can be feasible and effective. We also found that the wireless networks performed sparely better when UABSs are deployed at the height of $25 \mathrm{~m}$ compared to $36 \mathrm{~m}$ and $50 \mathrm{~m}$ deployment height 


\section{BIBLIOGRAPHY}

[1] Anritsu Corp., "Effectively Testing $700 \mathrm{MHz}$ Public safety LTE Broadband and P25 Narrowband Networks," tech. rep., Jan. 2013.

[2] Motorola Solutions, "Motorola Solutions Inc., APCO-25 Products," tech. rep.

[3] Kenwood, "Kenwood USA, LMR Products," tech. rep.

[4] Harris Corp., "Harris Corp., Products and Services for Public Safety and Professional Communications," tech. rep.

[5] Motorola Solutions, "Motorola Solutions Inc., TETRA Public Safety," tech. rep.

[6] Sepura, "Sepura plc, TETRA Products," tech. rep.

[7] Hytera, "Hytera, TETRA Products," tech. rep.

[8] FCC, "FCC website," tech. rep.

[9] FCC, "Improving Public Safety Communications in the $800 \mathrm{MHz}$ Band," tech. rep., Aug. 2004. Release FCC 04-168.

[10] U.S. Department Of Commerce, National Telecommunications and Information Administration. Office of Spectrum Management, "United States Frequency Allocations: The Radio Spectrum Chart," Aug. 2011.

[11] H. Schulzrinne, "It's all about spectrum," Feb. 2012. 2nd Annual MobileGov Summit.

[12] E. Olbrich, "Public Safety Communications Research Public Safety LTE," 2013. 
[13] FirstNet, "FirstNet website," tech. rep.

[14] Harris Corporation, "Converging Technologies," Channels Magazine, vol. 12, Aug. 2012.

[15] 3GPP, "Delivering Public Safety Communications with LTE," Jul. 2013.

[16] Centre Tecnològic de Telecomunicacions de Catalunya, "LENA Documentation," Dec. 2013.

[17] SDRSharp, "SDRSharp webpage," tech. rep.

[18] Great Scott Gadgets, "Great Scott Gadgets," tech. rep.

[19] Realtek, "Realtek," tech. rep.

[20] Evrytania LLC, "Evrytania LLC webpage," tech. rep.

[21] T. L. Doumi, "Spectrum Considerations for Public Safety in the United States," IEEE Commun. Mag., vol. 44, no. 1, pp. 30-37, 2006.

[22] M. M. Buddhikot and K. Ryan, "Spectrum Management in Coordinated Dynamic Spectrum Access Based Cellular Networks," in Proc. IEEE Int. Symp., New Frontiers in Dynamic Spectrum Access Networks, pp. 299-307, Nov. 2005.

[23] T. Kamakaris, M. M. Buddhikot, and R. Iyer, "A Case for Coordinated Dynamic Spectrum Access in Cellular Networks," in Proc. IEEE Int. Symp., New Frontiers in Dynamic Spectrum Access Networks, pp. 289-298, Nov. 2005.

[24] J. M. Peha, "Fundamental reform in public safety communications policy," Fed. Comm. LJ, vol. 59, p. 517, 2006. 
[25] N. Jesuale and B. C. Eydt, "A Policy Proposal to Enable Cognitive Radio for Public Safety and Industry in the Land Mobile Radio Bands," in Proc. IEEE Int. Symp., New Frontiers in Dynamic Spectrum Access Networks., pp. 66-77, Apr. 2007.

[26] T. A. Horan and B. L. Schooley, "Time-critical Information Services," Communications of the ACM, vol. 50, no. 3, pp. 73-78, 2007.

[27] J. Wang, M. Ghosh, and K. Challapali, "Emerging cognitive radio applications: A survey," IEEE Commun. Mag., vol. 49, no. 3, pp. 74-81, 2011.

[28] B. Le, F. A. Rodriguez, Q. Chen, B. P. Li, F. Ge, M. ElNainay, T. W. Rondeau, and C. W. Bostian, "A Public Safety Cognitive Radio Node," in Proc. SDR Forum Technical Conference (SDRF), Nov. 2007.

[29] A. K. Salkintzis, "Evolving Public Safety Communication Systems by Integrating WLAN and TETRA Networks," IEEE Commun. Mag., vol. 44, no. 1, pp. 38-46, 2006.

[30] A. Kumbhar, F. Koohifar, I. Guvenc, and B. Mueller, "A survey on legacy and emerging technologies for public safety communications," IEEE Commun. Survery Tuts., vol. 18, pp. 97-124, Sep. 2016.

[31] D. Athukoralage, I. Guvenc, W. Saad, and M. Bennis, "Regret based learning for UAV assisted LTE-U/WiFi public safety networks," in Proc. IEEE Global Commun. Conf. (GLOBECOM), (Washington, DC), pp. 1-7, Dec. 2016.

[32] J. Atkinson, "Motorola Solutions and EE confirmed for new UK Emergency Services Network," Wireless Magazine, Dec. 2015. 
[33] A. Kumbhar and I. Güvenç, "A comparative study of Land Mobile Radio and LTE-based public safety communications," in Proc. IEEE SoutheastCon 2015, (Fort Lauderdale, Florida), pp. 1-8, Jun. 2015.

[34] S. Chandrasekharan, K. Gomez, A. Al-Hourani, S. Kandeepan, T. Rasheed, L. Goratti, L. Reynaud, D. Grace, I. Bucaille, T. Wirth, et al., "Designing and implementing future aerial communication networks," IEEE Commun. Mag., vol. 54, no. 5, pp. 26-34, 2016.

[35] A. Merwaday, A. Tuncer, A. Kumbhar, and I. Guvenc, "Improved Throughput Coverage in Natural Disasters: Unmanned Aerial Base Stations for PublicSafety Communications," IEEE Vehic. Technol. Mag., vol. 11, pp. 53-60, Dec. 2016.

[36] A. Kumbhar, I. Güvenç, S. Singh, and A. Tuncer, "Exploiting LTE-Advanced HetNets and FeICIC for UAV-assisted public safety communications," IEEE Access, vol. 6, pp. 783-796, 2018.

[37] AT\&T, "Flying cow connects puerto rico," tech. rep., Nov. 2017.

[38] The Drive, "AT\&T and Verizon Test 4G LTE Drones in New Jersey," tech. rep., Jun. 2018.

[39] CNBC, "AT\&T and Verizon drones provide cell service in natural disasters," tech. rep., Aug. 2018.

[40] The Drive, "Drone Saves Man's Life From Kilauea Volcano Disaster in Hawaii," tech. rep., Jun. 2018. 
[41] H. Menouar, I. Guvenc, K. Akkaya, A. S. Uluagac, A. Kadri, and A. Tuncer, "UAV-enabled intelligent transportation systems for the smart city: Applications and challenges," IEEE Commun. Mag., vol. 55, no. 3, pp. 22-28, 2017.

[42] F. Koohifar, A. Kumbhar, and I. Guvenc, "Receding horizon multi-uav cooperative tracking of moving rf source," IEEE Commun. Let., vol. 21, no. 6, pp. 1433-1436, 2017.

[43] N. Saputro, K. Akkaya, and S. Uluagac, "Supporting Seamless Connectivity in Drone-assisted Intelligent Transportation Systems," in Proc. IEEE Local Computer Networks Workshops (LCN Workshops), (Chicago, IL), pp. 110116, Oct. 2018.

[44] H. Niu, N. Gonzalez-Prelcic, and R. W. Heath, "A uav-based traffic monitoring system-invited paper," in Proc. IEEE Vehic. Tech. Conf. (VTC Spring), (Porto, Portugal), pp. 1-5, Jun. 2018.

[45] B. Van der Bergh, A. Chiumento, and S. Pollin, "LTE in the sky: Trading off propagation benefits with interference costs for aerial nodes," IEEE Commun. Mag., vol. 54, no. 5, pp. 44-50, 2016.

[46] R. Amorim, H. Nguyen, J. Wigard, I. Z. Kovács, T. B. Sørensen, D. Z. Biro, M. Sørensen, and P. Mogensen, "Measured uplink interference caused by aerial vehicles in LTE cellular networks," IEEE Wireless Commun. Let., vol. 7, no. 6, pp. $958-961,2018$.

[47] A. Kumbhar, H. Binol, I. Guvenc, and K. Akkaya, "Interference Coordination for Aerial and Terrestrial Nodes in Three-Tier LTE-Advanced HetNet," in Proc. IEEE Radio Wireless Symp. (RWS), (Orlando, FL), pp. 1-4, Jan. 2019. 
[48] V. A. Dambal, S. Mohadikar, A. Kumbhar, and I. Guvenc, "Improving lora signal coverage in urban and sub-urban environments with uavs," in Proc. IEEE 2019 International Workshop on Antenna Technology (iWAT), (Miami, FL), pp. 210-213, 2019.

[49] V. Acuna, A. Kumbhar, E. Vattapparamban, F. Rajabli, and I. Guvenc, "Localization of wifi devices using probe requests captured at unmanned aerial vehicles," in Proc. IEEE Wireless Commun. Netw. Conf. (WCNC), (San Francisco, CA), pp. 1-6, Mar. 2017.

[50] A. Kumbhar, S. Singh, and I. Guvenc, "Uav assisted public safety communications with lte-advanced hetnets and feicic," in Proc. IEEE 28th Annual International Symposium on Personal, Indoor, and Mobile Radio Communications (PIMRC), pp. 1-7, 2017.

[51] A. Kumbhar, H. Binol, S. Singh, I. Guvenc, and K. Akkaya, "Heuristic Approach for Jointly Optimizing FeICIC and UAV Locations in Multi-Tier LTEAdvanced Public Safety HetNet," arXiv preprint arXiv:, 2019. Submitted to IET Cyber-systems and Robotics.

[52] S. Singh, A. Kumbhar, I. Güvenç, and M. L. Sichitiu, "Distributed Approaches for Inter-cell Interference Coordination in UAV-based LTE-Advanced HetNets," in Proc. IEEE 88th Vehic. Technol. Conf. (VTC-Fall), (Chicago, IL), pp. 1-6, Aug. 2019.

[53] Federal Communications Commission (FCC), "WT Docket 06-150, PS Docket 06-229, and WP Docket No. 07-100. In the Matter of Service Rules for the 698-746, 747-762 and 777-792 MHz Bands. Implementing a Nation- 
wide,Broadband, Interoperable PublicSafety Network in the $700 \mathrm{MHz}$ Band. Amendment of Part 90 of the Commission's Rules," tech. rep., June 2011.

[54] M. Yuksel, I. Guvenc, W. Saad, and N. Kapucu, "Pervasive spectrum sharing for public safety communications," IEEE Commun. Mag., vol. 54, no. 3, pp. 22-29, 2016.

[55] C. Gibbs, "Verizon claims largest small cell deployment in the U.S," Fierce Wireless, Mar. 2017.

[56] Y. Heisler, "AT\&T wants to use drones to improve its LTE network," Yahoo Tech. News, July. 2016.

[57] T. Nakamura, S. Nagata, A. Benjebbour, Y. Kishiyama, T. Hai, S. Xiaodong, Y. Ning, and L. Nan, "Trends in small cell enhancements in LTE advanced," IEEE Commun. Mag., vol. 51, no. 2, pp. 98-105, 2013.

[58] A. Merwaday, S. Mukherjee, and I. Güvenç, "Capacity analysis of LTEAdvanced HetNets with reduced power subframes and range expansion," EURASIP J. Wireless Commun. Netw., pp. 1-19, Nov. 2014.

[59] L. K. Moore, The first responder network (FirstNet) and next-generation communications for public safety: Issues for congress. Congressional Research Service, 2014.

[60] A. Z. Shifat, M. Z. Chowdhury, and Y. M. Jang, "Game-Based Approach for QoS Provisioning and Interference Management in Heterogeneous Networks," IEEE Access, vol. PP, no. 99, 2017. 
[61] A. Paulson and T. Schwengler, "A Review of Public Safety Communications, from LMR to voice over LTE (VoLTE)," in Proc. IEEE Personal Indoor and Mobile Radio Comm. (PIMRC), pp. 3513-3517, Sep. 2013.

[62] G. Baldini, S. Karanasios, D. Allen, and F. Vergari, "Survey of Wireless Communication Technologies for Public Safety," IEEE Commun. Surv. Tut., vol. 16, no. 2, pp. 619-641, 2014.

[63] B. Haupt, N. Kapucu, M. Yuksel, I. Guvenc, and W. Saad, "On the Evolution of Wireless Communication Technologies and Spectrum Sharing for Public Safety," Risks, Hazards, and Crisis in Public Policy, 2016.

[64] T. Doumi, M. F. Dolan, S. Tatesh, A. Casati, G. Tsirtsis, K. Anchan, and D. Flore, "LTE for public safety networks," IEEE Commun. Mag., vol. 51, no. 2, pp. 106-112, 2013.

[65] "LTE: The Technology Driver for Future Public Safety Communications,"

[66] Shared Spectrum Company, "Cognitive Radio Technology," Air Force SBIR/STTR Innovation Story, Jan. 2012.

[67] Australian Communications and Media Authority (ACMA), "Spectrum after scarcity: joint ACMA \& Swinburne event," Spectrum tune-up: Spectrum after scarcity, Oct. 2015.

[68] K. Pretz, "Overcoming Spectrum Scarcity," IEEE, The Institute, Aug. 2012.

[69] A. Merwaday, M. Yuksel, T. Quint, I. Guvenc, W. Saad, and N. Kapucu, "Incentivizing Spectrum Sharing via Subsidy Regulations," arXiv preprint arXiv:1411.5302, 2014. 
[70] M. Yuksel, T. Quint, I. Guvenc, W. Saad, and N. Kapucu, "Fostering wireless spectrum sharing via subsidization," in Proc. IEEE Allerton Conf. Commun., pp. 1192-1199, Oct. 2013.

[71] "Auction of $700 \mathrm{MHz}$ band licenses scheduled for January 16, 2008 comment sought on competitive bidding procedures for Auction 73," tech. rep., Aug. 2007.

[72] D. Wyllie, "The D-Block, public safety, and a tipping point," FireRescue1, Sep. 2010.

[73] R. Hallahan and J. M. Peha, "Enabling Public Safety Priority Use of Commercial Wireless Networks," Homeland Security Affairs, vol. 9, p. 13, 2013.

[74] FCC, "Third Report and Order and Fourth Further Notice of Proposed Rulemaking, WT Docket No. 06-150, PS Docket No. 06-229, WP Docket No. 07100," Comments Of The National Public Safety Telecommunications Council, Jan. 2011.

[75] C. Essid, "Nationwide Public Safety Broadband Network," Homeland Security, Office Of Emergency Communications, Jun. 2012.

[76] FCC, "Public Safety And Homeland Security Bureau," tech. rep.

[77] R. Mussenden, "VHF/UHF Narrowbanding Information for Public Safety Licensees," Public Safety and Homeland Security Bureau (PSHSB), Workshop on VHF/UHF narrowbanding, Jan. 2011.

[78] FCC, "FCC rule part 90 private land mobile radio services," tech. rep. e-CFR Data is current as of February 5, 2015.

[79] Columbia University Amateur Radio Club, "FCC band plan," tech. rep. 
[80] RadioReference.com, "State of Florida Radio Reference - Trunked Systems," tech. rep.

[81] NPSTC, "T-Band Report," A NPSTC Public Safety Communications Report, Mar. 2013.

[82] Parker Fire Protection District, "Colorado 4.9 GHz Project," Apr. 2006.

[83] Mission Critical Solutions (MCS), "Hollywood, FL WiFi Project,"

[84] Rinehart, Bette and Eierman, David, " 4.9 GHz Public Safety Broadband Spectrum. Overview of Technical Rules And Step-By-Step Licensing Instructions ," tech. rep., Motorola, Inc, Jul. 2010.

[85] J. M. Peha, "Regulatory and Policy Issues Protecting Public Safety with Better Communications Systems," IEEE Commun. Mag., vol. 43, no. 3, pp. 10-11, 2005.

[86] J. Peha, W. Johnston, P. Amodio, and T. Peters, "The Public Safety Nationwide Interoperable Broadband Network: A New Model for Capacity, Performance and Cost," tech. rep., 2010.

[87] G. Iapichino, D. Câmara, C. Bonnet, and F. Filali, Public Safety Networks. IGI Global, 2011.

[88] ETSI, "Terrestrial Trunked Radio (TETRA); Release 2; Designer's Guide; TETRA High-Speed Data (HSD); TETRA Enhanced Data Service (TEDS)," Oct. 2007. ETSI TR 102580 V1.1.1 (2007-10).

[89] ETSI, "Electromagnetic compatibility and Radio spectrum Matters (ERM); TETRA Air-Ground-Air services (AGA); System reference document," May. 2006. ETSI TR 102459 V1.1.1 (2006-05). 
[90] J. M. Vanderau, "NTIA Technical Report TR-99-358 Delivered Audio Quality Measurements on Project 25 Land Mobile Radios," tech. rep., Nov. 1998.

[91] R. I. Desourdis, Emerging public safety wireless communication systems. Artech House, 2002.

[92] M. Portmann and A. A. Pirzada, "Wireless Mesh Networks for Public Safety and Crisis Management Applications," IEEE Internet Computing, vol. 12, no. 1, pp. 18-25, 2008.

[93] W. R. M. III, "How Project 25 two-slot TDMA works," Urgent Communications, Jan. 2008.

[94] TIA STANDARD, "Project 25 FDMA - Common Air Interface," New Technology Standards Project - Digital Radio Technical Standards, Sept. 2003. TIA-102.BAAA-A Revision of TIA-102.BAAA.

[95] Aeroflex, Inc., "Application Note: Understanding P25 Modulation Fidelity," tech. rep., Jan. 2010.

[96] Aeroflex Inc., "Understanding Advanced P25 Control Channel Functions," tech. rep., Aug. 2006.

[97] RadioReference.com, "APCO Project 25,"

[98] Washington Update, "PAHPA, Senate Passes, and E.M.S. Supporters.," Apr. 2012. Middle Class Tax Relief and Job Creation Act of 2012, (H.R. 3630).

[99] A. M. Seybold, "Commercial Networks and First Responders," Andrew Seybold Media, Feb. 2007. 
[100] D. Jackson, "Obama makes it official, signs D Block legislation," Urgent Communications, Feb. 2012.

[101] Australian Communications and Media Authority (ACMA), "Spectrum for public safety radiocommunications Current ACMA initiatives and decisions," Oct. 2012 .

[102] J. Atkinson, "WRC-15 recommends reserving spectrum for public safety broadband," Wireless Magazine, Nov. 2015.

[103] European Conference of Postal and Telecommunications Administrations (CEPT) Electronic Communications Committee (ECC), "User requirements and spectrum needs for future European broadband PPDR systems (Wide Area Networks)," ECC Report 199, May 2013.

[104] Industry Canada, "Gazette Notice SMSE-007-12 - Consultation on a Policy, Technical and Licensing Framework for Use of the Public Safety Broadband Spectrum in the Bands 758-763 MHz and 788-793 MHz (D Block) and 763-768 MHz and 793-798 MHz (PSBB Block)," Spectrum Management and Telecommunications, Aug. 2012.

[105] FCC News Release, "FCC Takes Action to Promote Interoperability in the Lower 700 MHz Band," Oct. 2013.

[106] G. Araniti, C. Campolo, M. Condoluci, A. Iera, and A. Molinaro, "LTE for Vehicular Networking: A Survey," IEEE Commun. Mag., vol. 51, no. 5, pp. 148$157,2013$.

[107] K. Balachandran, K. C. Budka, T. P. Chu, T. L. Doumi, and J. H. Kang, "Mobile Responder Communication Networks for Public Safety," IEEE Commun. Mag., vol. 44, no. 1, pp. 56-64, 2006. 
[108] D. FREDEZ, "Voice over LTE: PTT, Timing and Reality," The International Wireless Communications Expo (IWCE), Mar. 2013. Thales Communications, Inc.

[109] M. Solanki, S. Salehi, and A. Esmailpour, "LTE Security: Encryption Algorithm Enhancements," in Proc. ASEE Northeast Section Conf., Mar. 2013.

[110] M. Simsek, A. Merwaday, N. Correal, and I. Guvenc, "Device-to-Device Discovery based on 3GPP System Level Simulations," in Proc. IEEE Globecom Workshops (GC Wkshps), pp. 555-560, Dec. 2013.

[111] L. Babun, "Extended Coverage for Public Safety and Critical Communications Using Multi-hop and D2D Communications," Master's thesis, Florida International University, 2015.

[112] L. Babun, M. Simsek, and I. Guvenc, "Intercell Interference Coordination for D2D Discovery in LTE-A HetNets," in Proc. IEEE Wireless Communications and Networking Conference (WCNC), pp. 2202-2207, Apr. 2014.

[113] K. C. Budka, T. Chu, T. L. Doumi, W. Brouwer, P. Lamoureux, and M. E. Palamara, "Public Safety Mission Critical Voice Services over LTE," Bell Labs Technical Journal, vol. 16, no. 3, pp. 133-149, 2011.

[114] K. Georgiev and D. Dimitrova, "Impact of Relaying on Inter-cell Interference in Mobile Cellular Networks," in Proc. European Wireless Conference (EW), pp. 398-405, Apr. 2010.

[115] M. Castaneda, M. T. Ivrlač, J. Nossek, I. Viering, and A. Klein, "On Downlink Intercell Interference in a Cellular System," in Proc. IEEE Symp. Personal, Indoor and Mobile Radio Communications (PIMRC), pp. 1-5, Sep. 2007. 
[116] V. Pauli and E. Seidel, "Inter-Cell Interference Coordination for LTE-A," White Paper, Nomor Research GmbH, Munich, Germany, 2011.

[117] S. Wendelken, "Mission-Critical PTT Set for LTE Release 13, Release 12 Freeze Likely in 2015," Sep. 2014. Radio Resource Magazine.

[118] D. Flore, "Evolution of LTE in Release 13," Feb. 2015.

[119] Motorola, Inc., "Why migrate to P25 digital LMR. building safer, more resilient communities in a new era of LMR intelligence," tech. rep., 2014.

[120] Motorola Solutions, Inc., "VALR ${ }^{\mathrm{TM}}$ Mission Critical Architecture," tech. rep., 2013.

[121] W. Wang, Y. Chen, Q. Zhang, and T. Jiang, "A software-defined wireless networking enabled spectrum management architecture," IEEE Commun. Mag., vol. 54, no. 1, pp. 33-39, 2016.

[122] RTL-SDR.COM, "RTL-SDR Tutorial: Decoding Digital Voice (P25, DMR, NXDN, D-STAR) with DSD," Apr. 2013.

[123] F. Franz, "SDR for Public Safety," tech. rep., L-3 Communications.

[124] D. Mohney, "SDR can improve public-safety radios," tech. rep., Urgent Communications, Oct. 2005.

[125] A. A. Abidi, "The Path to the Software-Defined Radio Receiver," IEEE J. Solid-State Circuits, vol. 42, no. 5, pp. 954-966, 2007.

[126] G. Youngblood, "A Software-Defined Radio for the Masses, Part 1," tech. rep., American Radio Relay League. 
[127] R. FerrÃos and O. Sallent, Mobile Broadband Communications for Public Safety: The Road Ahead Through LTE Technology. John Wiley \& Sons, 2015.

[128] A. M. Wyglinski, D. P. Orofino, M. N. Ettus, and T. W. Rondeau, "Revolutionizing software defined radio: case studies in hardware, software, and education," IEEE Commun. Mag., vol. 54, no. 1, pp. 68-75, 2016.

[129] Proxmark, "HackRF Tutorial," tech. rep.

[130] I. Güvenç, U. C. Kozat, M.-R. Jeong, F. Watanabe, and C.-C. Chong, "Reliable multicast and broadcast services in relay-based emergency communications," IEEE Wireless Commun., vol. 15, no. 3, pp. 40-47, 2008.

[131] J. Ross, "Public Safety Technology: The Truth About LTE," tech. rep., NPSTC.

[132] W. Brouwer, "eMBMS," tech. rep., May 2013.

[133] J. F. Monserrat, J. Calabuig, A. Fernandez-Aguilella, and D. GomezBarquero, "Joint delivery of unicast and E-MBMS services in LTE networks," IEEE Trans. Broadcast., vol. 58, no. 2, pp. 157-167, 2012.

[134] 3GPP, "Multimedia Broadcast/Multicast Service (MBMS) user services; Stage 1," TS 22.246, Ver.12.0.0, Ver.13.0.0, 3rd Generation Partnership Project (3GPP).

[135] L. Frenzel, "Millimeter Waves Will Expand The Wireless Future," tech. rep., Electronic Design, Mar. 2013.

[136] A. Bleicher, "Millimeter Waves May Be the Future of 5G Phones," IEEE Spectrum, pp. 11-12, 2013. 
[137] T. Rappaport, W. Roh, and K. Cheun, "Smart Antennas Could Open Up New Spectrum For 5G," IEEE Spectrum, pp. 11-12, Aug. 2014.

[138] A. I. Sulyman, A. T. Nassar, M. K. Samimi, G. R. MacCartney, T. S. Rappaport, and A. Alsanie, "Radio Propagation Path Loss Models for 5G Cellular Networks in the $28 \mathrm{GHz}$ and $38 \mathrm{GHz}$ Millimeter-Wave Bands," IEEE Commun. Mag., vol. 52, no. 9, pp. 78-86, 2014.

[139] Y. Niu, Y. Li, D. Jin, L. Su, and A. V. Vasilakos, "A survey of millimeter wave communications (mmWave) for 5G: opportunities and challenges," Wireless Networks, vol. 21, no. 8, pp. 2657-2676, 2015.

[140] V. Thomas, M. El-Hajjar, and L. Hanzo, "Millimeter-wave radio over fiber optical upconversion techniques relying on link non-linearity," IEEE Commun. Surv. Tut., vol. 18, no. 1, pp. 29-53, 2016.

[141] S. Kutty and D. Sen, "Beamforming for Millimeter Wave Communications: An Inclusive Survey," IEEE Commun. Surv. Tut., 2015.

[142] M. R. Akdeniz, Y. Liu, M. K. Samimi, S. Sun, S. Rangan, T. S. Rappaport, and E. Erkip, "Millimeter Wave Channel Modeling and Cellular Capacity Evaluation," IEEE J. Sel. Areas Commun., vol. 32, no. 6, pp. 1164-1179, 2014.

[143] H. Mopidevi, D. Rodrigo, O. Kaynar, L. Jofre, and B. A. Cetiner, "Compact and broadband antenna for LTE and public safety applications," IEEE Antennas and Wireless Propag. Lett., vol. 10, pp. 1224-1227, 2011.

[144] T. L. Marzetta, "Noncooperative Cellular Wireless with Unlimited numbers of Base Station Antennas," IEEE Trans. Wireless Commun., vol. 9, no. 11, pp. $3590-3600,2010$. 
[145] J. Jose, A. Ashikhmin, P. Whiting, and S. Vishwanath, "Channel Estimation and Linear Precoding in Multiuser Multiple-Antenna TDD Systems," IEEE Trans. Vehic. Technol., vol. 60, no. 5, pp. 2102-2116, 2011.

[146] B. Gopalakrishnan and N. Jindal, "An Analysis of Pilot Contamination on Multi-user MIMO Cellular Systems with many Antennas," in Proc. IEEE Int. Workshop on Signal Processing Advances in Wireless Communications (SPAWC), pp. 381-385, Jun. 2011.

[147] O. Elijah, C. Leow, T. Rahman, S. Nunoo, and S. Iliya, "A Comprehensive Survey of Pilot Contamination in Massive MIMO-5G System," IEEE Commun. Surv. Tut., vol. 99, Nov. 2015.

[148] E. G. Larsson, O. Edfors, F. Tufvesson, and T. L. Marzetta, "Massive MIMO for Next Generation Wireless Systems," arXiv preprint arXiv:1304.6690, 2013.

[149] T. Nakamura, S. Nagata, A. Benjebbour, Y. Kishiyama, T. Hai, S. Xiaodong, Y. Ning, and L. Nan, "Trends in Small Cell Enhancements in LTE Advanced," IEEE Commun. Mag., vol. 51, no. 2, pp. 98-105, 2013.

[150] A. Merwaday and I. Guvenc, "UAV Assisted Heterogeneous Networks for Public Safety Communications," in Proc. IEEE Wireless Commun. and Networking Conference Workshops (WCNCW), pp. 329-334, Mar. 2015.

[151] Qualcomm Incorporated, "A Comparison of LTE Advanced HetNets and WiFi," tech. rep., Oct. 2011.

[152] B. A. Bjerke, "LTE-Advanced and the Evolution of LTE Deployments," IEEE Wireless Commun., vol. 18, no. 5, pp. 4-5, 2011. 
[153] 3GPP, RWS-120045, "Summary of 3GPP TSG-RAN Workshop on Release 12 and Onward," Jun. 2012.

[154] Qualcomm, "Extending benefits of LTE Advanced to Unlicensed Spectrum," Apr. 2014.

[155] Y. Sui, I. Guvenc, and T. Svensson, "Interference management for moving networks in ultra-dense urban scenarios," EURASIP Journal on Wireless Communications and Networking, no. 1, pp. 1-32, 2015.

[156] W. C. Bennett, Civilian Drones, Privacy, and the Federal-State Balance. 2014.

[157] M. Hainzl, "Integrating UAVs into Public Safety LTE Networks," tech. rep., 2014.

[158] X. Li, D. Guo, H. Yin, and G. Wei, "Drone-assisted Public Safety Wireless Broadband Network," in Proc. IEEE Wireless Communications and Networking Conf. Workshops (WCNCW), pp. 361-368, Mar. 2015.

[159] K. Gomez, T. Rasheed, L. Reynaud, and I. Bucaille, "Realistic deployments of LTE-based Hybrid Aerial-Terrestrial Networks for public safety," in Proc. IEEE 18th International Workshop on Computer Aided Modeling and Design of Communication Links and Networks (CAMAD), pp. 233-237, Sep. 2013.

[160] V. Wolfe, W. Frobe, V. Shrinivasan, T.-Y. Hsieh, and H. M. Gates, "Feasibility Study of Utilizing 4G LTE Signals in Combination with Unmanned Aerial Vehicles for the Purpose of Search and Rescue of Avalanche Victims (Increment 1)," Research Report, University of Colorado at Boulder, Boulder, CO., 2014. 
[161] K. Gomez, T. Rasheed, L. Reynaud, and S. Kandeepan, "On the Performance of Aerial LTE base-stations for Public Safety and Emergency Recovery," in Proc. IEEE Globecom Workshops, pp. 1391-1396, Dec. 2013.

[162] A. Merwaday, A. Tuncer, A. Kumbhar, and I. Guvenc, "Unmanned Aerial Base Stations for Public Safety Communications," IEEE Vehic. Technol. Mag., 2016.

[163] K. Gomez, A. Hourani, L. Goratti, R. Riggio, S. Kandeepan, and I. Bucaille, "Capacity evaluation of Aerial LTE base-stations for public safety communications," in Proc. IEEE European Conference on Networks and Communications (EuCNC), pp. 133-138, Jun. 2015.

[164] L. Gupta, R. Jain, and G. Vaszkun, "Survey of Important Issues in UAV Communication Networks," IEEE Commun. Surv. Tut., vol. 99, 2015.

[165] Y. Saleem, M. H. Rehmani, and S. Zeadally, "Integration of cognitive radio technology with unmanned aerial vehicles: issues, opportunities, and future research challenges," Journal of Network and Computer Applications, vol. 50, pp. 15-31, 2015.

[166] W. Khawja, I. Guvenc, and D. W. Matolak, "UWB Channel Sounding and Modeling for UAV Air-to-Ground Propagation Channels," in Proc. IEEE Global Telecommun. Conf. (GLOBECOM), Washington, DC, Dec. 2016.

[167] N. Rupasinghe, A. S. Ibrahim, and I. Guvenc, "Optimum Hovering Locations with Angular Domain User Separation for Cooperative UAV Networks," in Proc. IEEE Global Telecommun. Conf. (GLOBECOM), Washington, DC, Dec. 2016. 
[168] J. Fingas, "Alcatel-Lucent flies Parrot AR.Drone 2.0 over 3,280 feet using LTE: reach out and buzz someone (video)," tech. rep., Engadget, Oct. 2012.

[169] K. Daniel, B. Dusza, A. Lewandowski, and C. Wietfeld, "AirShield: A systemof-systems MUAV Remote Sensing Architecture for Disaster Response," in Proc. IEEE Annual Systems Conf., pp. 196-200, Mar. 2009.

[170] Z. Alazawi, S. Altowaijri, R. Mehmood, and M. B. Abdljabar, "Intelligent Disaster Management System Based on Cloud-Enabled Vehicular Networks," in Proc. IEEE Int. Conf. ITS Telcommun. (ITST), pp. 361-368, Aug. 2011.

[171] M. Eltoweissy, S. Olariu, and M. Younis, "Towards Autonomous Vehicular Clouds," in Ad hoc Networks, pp. 1-16, Springer, 2010.

[172] The FirstNet CTO Devices Team, "Tech Talk: Updated Vehicular Network System Vision," tech. rep., FirstNet, Apr. 2016.

[173] Nokia Networks, "LTE-Advanced Evolution in Releases 12 - 14. New services to pave the way to $5 \mathrm{G}$," tech. rep., Nokia.

[174] W. Chen, Vehicular Communications and Networks: Architectures, Protocols, Operation and Deployment. Elsevier, 2015.

[175] 3GPP, "Study on LTE support for V2X services," Tech. Rep. SP-150051, 3rd Generation Partnership Project (3GPP).

[176] 3GPP, "Outgoing Liaison Statement on V2X study in 3GPP," Tech. Rep. SP-150166, 3rd Generation Partnership Project (3GPP).

[177] Qualcomm Incorporated, "V2X Communication in 3GPP," Tech. Rep. S1144374, 3rd Generation Partnership Project (3GPP). 
[178] S. Antipolis, "A look at the Requirements for LTE in the Unlicensed Bands," 3GPP TSG RAN Workshop on LTE in unlicensed spectrum, RWS-140006, Jun. 2014. Source: InterDigital Communications.

[179] NTT DOCOMO, "Views on licensed-assisted access (LAA) for unlicensed spectrum - scenarios and initial evaluation results," 3GPP News/New Opportunities for 3GPP in Rel-12, Jun. 2014.

[180] N. Rupasinghe and I. Guvenc, "Reinforcement Learning for Licensed-Assisted Access of LTE in the Unlicensed Spectrum," in Proc. IEEE Wireless Communications and Networking Conference (WCNC), pp. 1279-1284, Mar. 2015.

[181] N. Rupasinghe and I. Guvenc, "Licensed-Assisted Access for WiFi-LTE Coexistence in the Unlicensed Spectrum," in Proc. IEEE Globecom Workshops (GC Wkshps), pp. 894-899, Dec. 2014.

[182] Huawei Technologies Co., Ltd, "LTE Small Cell v.s. WiFi User Experience," tech. rep., 2013.

[183] D. Athukorala, I. Guvenc, W. Saad, and M. Bennis, "Regret Based Learning for UAV assisted LTE-U/WiFi Public Safety Networks," in Proc. IEEE Global Telecommun. Conf. (GLOBECOM), Washington, DC, Dec. 2016.

[184] S. Antipolis, "Use Cases and Scenarios for Licensed Assisted Access," 3GPP TSG RAN Workshop on LTE in unlicensed spectrum, RWS-140020, Jun. 2014. Source: Verizon, CMCC, Huawei, Ericsson.

[185] S. Antipolis, "Extending the Benefits of LTE to Unlicensed Spectrum," $3 G P P$ TSG RAN Workshop on LTE in unlicensed spectrum, RWS-140008, Jun. 2014. Source: Qualcomm. 
[186] S. Antipolis, "Requirements and Proposed Coexistence Topics for the LTE-U Study," 3GPP TSG RAN Workshop on LTE in unlicensed spectrum, RWS140010, Jun. 2014. Source: Sony Mobile Communications.

[187] S. Antipolis, "Co-existence Considerations for LTE-U," 3GPP TSG RAN Workshop on LTE in unlicensed spectrum, RWS-140025, Jun. 2014. Source: Cisco Systems.

[188] S. Antipolis, "Consideration of LTE in Unlicensed Spectrum," 3GPP TSG RAN Workshop on LTE in unlicensed spectrum, RWS-140028, Jun. 2014. Source: Fujitsu.

[189] S. Antipolis, "LTE in Unlicensed Spectrum," 3GPP TSG RAN Workshop on LTE in unlicensed spectrum, RWS-140018, Jun. 2014. Source: Intel Corporation.

[190] M. Yuksel, I. Guvenc, W. Saad, and N. Kapucu, "Pervasive Spectrum Sharing for Public Safety Communications," IEEE Commun. Mag., 2016.

[191] S. Ghafoor, P. Sutton, C. Sreenan, and K. Brown, "Cognitive radio for disaster response networks: survey, potential, and challenges," IEEE Wireless Commun., vol. 21, no. 5, pp. 70-80, 2014.

[192] F. Akhtar, M. H. Rehmani, and M. Reisslein, "White space: Definitional perspectives and their role in exploiting spectrum opportunities," Telecommunications Policy, vol. 40, no. 4, pp. 319-331, 2016.

[193] A. H. Sakr and E. Hossain, "Cognitive and energy harvesting-based d2d communication in cellular networks: Stochastic geometry modeling and analysis," IEEE Trans. Commun., vol. 63, no. 5, pp. 1867-1880, 2015. 
[194] E. Hossain, D. Niyato, and Z. Han, Dynamic spectrum access and management in cognitive radio networks. Cambridge university press, 2009.

[195] E. Hossain and V. K. Bhargava, Cognitive wireless communication networks. Springer Science \& Business Media, 2007.

[196] L. Gomez, A. Laube, and C. Ulmer, "Secure sensor networks for public safety command and control system," in Proc. IEEE Conf. Technologies for Homeland Security (HST), pp. 59-66, May 2009.

[197] L. Gomez and C. Ulmer, "Secure sensor networks for critical infrastructure protection," in Proc. IEEE Int. Conf. Sensor Technologies and Applications (SENSORCOMM), pp. 144-150, Jul. 2010.

[198] O. B. Akan, O. B. Karli, and O. Ergul, "Cognitive radio sensor networks," IEEE Network, vol. 23, no. 4, pp. 34-40, 2009.

[199] G. Han, J. Jiang, C. Zhang, T. Duong, M. Guizani, and G. Karagiannidis, "A survey on mobile anchor node assisted localization in wireless sensor networks," IEEE Commun. Surv. Tut., vol. 99, 2016.

[200] Y. Gu, F. Ren, Y. Ji, and J. Li, "The evolution of sink mobility management in wireless sensor networks: A survey," IEEE Commun. Surv. Tut., vol. 18, no. 1, pp. 507-524, 2016.

[201] S. H. R. Bukhari, M. H. Rehmani, and S. Siraj, "A Survey of Channel Bonding for Wireless Networks and Guidelines of Channel Bonding for Futuristic Cognitive Radio Sensor Networks," IEEE Commun. Surv. Tut., vol. 99, 2015.

[202] P. Smedley, "Examining IoT in Public Safety," Oct. 2014. 
[203] J. Teel, "Next-Gen Public Safety Technologies Using IoT," Wireless Design and Development Magazine, May 2015.

[204] B. Kantarci and H. T. Mouftah, "Trustworthy Sensing for Public Safety in Cloud-Centric Internet of Things," IEEE Internet of Things Journal, vol. 1, no. 4, pp. 360-368, 2014.

[205] J. Gubbi, R. Buyya, S. Marusic, and M. Palaniswami, "Internet of Things (IoT): A Vision, Architectural Elements, and Future Directions," Future Generation Computer Systems, vol. 29, no. 7, pp. 1645-1660, 2013.

[206] L. Atzori, A. Iera, and G. Morabito, "The Internet of Things: A Survey," Computer Networks, vol. 54, no. 15, pp. 2787-2805, 2010.

[207] Q.-B. Sun, J. Liu, S. Li, C.-x. FAN, and J.-j. SUN, "Internet of Things: Summarize on Concepts, Architecture and Key Technology Problem [J]," Journal of Beijing University of Posts and Telecommunications, vol. 3, no. 3, pp. 1-9, 2010.

[208] C. Neagle, "How the Internet of Things is Transforming Law Enforcement," NetworkWorld, Nov. 2014.

[209] A. R. McGee, M. Coutière, and M. E. Palamara, "Public Safety Network Security Considerations," Bell Labs Technical Journal, vol. 17, no. 3, pp. 79$86,2012$.

[210] N. Hastings and J. Franklin, "Consideration for Identity Management in Public Safety Mobile Networks," tech. rep., Mar. 2015.

[211] H. Ghafghazi, A. El Mougy, H. T. Mouftah, and C. Adams, "Classification of technological privacy techniques for LTE-based public safety networks," 
in Proc. 10th ACM symposium on QoS and security for wireless and mobile networks, pp. 41-50, ACM, Sep. 2014.

[212] E. Vattapparamban, I. Guvenc, A. Yurekli, K. Akkaya, and S. Uluagac, "Drones for Smart Cities: Issues in Cybersecurity, Privacy, and Public Safety," in Proc. IEEE IWCMC Smart Cities and Platforms, Cyprus, 2016.

[213] J. Wang, Y. Wu, N. Yen, S. Guo, and Z. Cheng, "Big Data Analytics for Emergency Communication Networks: A Survey," IEEE Commun. Surv. Tut., vol. 99, 2016.

[214] N. Saquib, E. Hossain, and D. I. Kim, "Fractional frequency reuse for interference management in LTE-advanced hetnets," IEEE Wireless Commun., vol. 20, no. 2, pp. 113-122, 2013.

[215] S. Deb, P. Monogioudis, J. Miernik, and J. P. Seymour, "Algorithms for enhanced inter-cell interference coordination (eICIC) in LTE hetnets," IEEE/ACM Trans. Nwk., vol. 22, no. 1, pp. 137-150, 2014.

[216] S. Mukherjee and I. Güvenç, "Effects of range expansion and interference coordination on capacity and fairness in heterogeneous networks," in Proc. IEEE Asilomar Conf. on Signals, Systems and Computers, pp. 1855-1859, 2011.

[217] A. Al-Hourani, S. Kandeepan, and S. Lardner, "Optimal LAP altitude for maximum coverage," IEEE Wireless Commun. Lett., vol. 3, no. 6, pp. 569$572,2014$.

[218] R. I. Bor-Yaliniz, A. El-Keyi, and H. Yanikomeroglu, "Efficient 3-D placement of an aerial base station in next generation cellular networks," in Proc. IEEE Intl. Conf. Commun. (ICC), (Kuala Lumpur, Malaysia), pp. 1-5, 2016. 
[219] M. Mozaffari, W. Saad, M. Bennis, and M. Debbah, "Optimal transport theory for power-efficient deployment of unmanned aerial vehicles," in Proc. IEEE Intl. Conf. Commun. (ICC), (Kuala Lumpur, Malaysia), pp. 1-6, 2016.

[220] V. Sharma, M. Bennis, and R. Kumar, "UAV-assisted heterogeneous networks for capacity enhancement," IEEE Commun. Lett., vol. 20, no. 6, pp. 12071210, 2016.

[221] M. Narang, S. Xiang, W. Liu, J. Gutierrez, L. Chiaraviglio, A. Sathiaseelan, and A. Merwaday, "UAV-assisted edge infrastructure for challenged networks,"

[222] E. Christy, R. P. Astuti, B. Syihabuddin, B. Narottama, O. Rhesa, and F. Rachmawati, "Optimum UAV flying path for Device-to-Device communications in disaster area," in Proc. IEEE Int. Conf. Sig Sys. (ICSigSys), (Bali, Indonesia), pp. 318-322, 2017.

[223] M. Mozaffari, W. Saad, M. Bennis, and M. Debbah, "Drone small cells in the clouds: Design, deployment and performance analysis," in Proc. IEEE Global Commun. Conf. (GLOBECOM), (San Diego, CA), pp. 1-6, 2015.

[224] Z. Kaleem and K. Chang, "Public Safety Priority-Based User Association for Load Balancing and Interference Reduction in PS-LTE Systems," IEEE Access, vol. 4, pp. 9775-9785, 2016.

[225] N. Rupasinghe, Y. Yapıcı, I. Güvenç, and Y. Kakishima, "Non-orthogonal multiple access for mmwave drone networks with limited feedback," IEEE Trans. Commun., vol. 67, no. 1, pp. 762-777, 2019. 
[226] Y. Sun, T. Wang, and S. Wang, "Location Optimization for Unmanned Aerial Vehicles Assisted Mobile Networks," in Proc. IEEE Intl. Conf. Commun. (ICC), (Kansas City, MO), pp. 1-6, May 2018.

[227] S. Hanna, H. Yan, and D. Cabric, "Distributed UAV Placement Optimization for Cooperative Line-of-sight MIMO Communications," in Proc. IEEE Intl. Conf. Acoustics, Speech, Signal Process. (ICASSP), (Brighton, UK), pp. 46194623, May 2019.

[228] U. Challita, W. Saad, and C. Bettstetter, "Interference Management for Cellular-Connected UAVs: A Deep Reinforcement Learning Approach," IEEE Trans. Wireless Commun., vol. 18, no. 4, pp. 2125-2140, 2019.

[229] Q. Zhang, M. Mozaffari, W. Saad, M. Bennis, and M. Debbah, "Machine learning for predictive on-demand deployment of UAVs for wireless communications," in Proc. IEEE Global Commun. Conf. (GLOBECOM), (Abu Dhabi, United Arab Emirates), pp. 1-6, Dec. 2018.

[230] Q. Zhang, W. Saad, M. Bennis, X. Lu, M. Debbah, and W. Zuo, "Predictive Deployment of UAV Base Stations in Wireless Networks: Machine Learning Meets Contract Theory," arXiv preprint arXiv:1811.01149, 2018.

[231] S. Sharafeddine and R. Islambouli, "On-demand deployment of multiple aerial base stations for traffic offloading and network recovery," Elsevier Computer Networks, vol. 156, pp. 52-61, 2019.

[232] P. Li and J. Xu, "Placement Optimization for UAV-Enabled Wireless Networks with Multi-Hop Backhauls," Springer J. Commun. Information Netw., vol. 3, no. 4, pp. 64-73, 2018. 
[233] A. Fouda, A. S. Ibrahim, Í. Güvenç, and M. Ghosh, "Interference management in uav-assisted integrated access and backhaul cellular networks," IEEE Access, vol. 7, pp. 104553-104566, 2019.

[234] M. M. Azari, F. Rosas, A. Chiumento, and S. Pollin, "Coexistence of terrestrial and aerial users in cellular networks," in Proc. IEEE Global Commun. Conf., (Singapore), pp. 1-6, Dec. 2017.

[235] P. Lunness, "P25 Radio Systems Training Guide," TG-001, pp. 1-0.

[236] Mesa, "MESA website," tech. rep.

[237] TCCA: The Critical Communications Association, "TETRA+Critical Communication Association website," tech. rep.

[238] J. Dunlop, D. Girma, and J. Irvine, Digital mobile communications and the TETRA system. John Wiley \& Sons, 2013.

[239] Acterna corp., "Tetra introduction," 2001. White paper.

[240] P. Mogensen, W. Na, I. Z. Kovács, F. Frederiksen, A. Pokhariyal, K. I. Pedersen, T. Kolding, K. Hugl, and M. Kuusela, "LTE capacity compared to the Shannon Bound," in Proc. IEEE Vehic. Technol. Conf. (VTC), pp. 1234-1238, Apr. 2007.

[241] E. Galloix, "On public safety LTE network: Simulation results in uplink from a video surveillance use case," in Proc. IEEE Int. Conf. 5G for Ubiquitous Connectivity (5GU), pp. 297-299, Nov. 2014.

[242] Cellular/Mobile Telecommunications, "LTE Frequency Bands and Spectrum Allocations," tech. rep., Radio-Electronics.com. 
[243] "Middle Class Tax Relief and Job Creation Act of 2012," Public Law 112-96, Feb. 2012.

[244] Etherstack, "Etherstack website," tech. rep.

[245] M. Sauter, From GSM to LTE: an introduction to mobile networks and mobile broadband. John Wiley \& Sons, 2010.

[246] J. Seefeldt, "LTE Comparison with Legacy Voice Land Mobile Radio (LMR) OEC/ICTAP Office of Emergency Communications," tech. rep., Aug. 2011. AR - System Comparison.

[247] DHS Office of Emergency Communications, "Guidelines for Encryption in Land Mobile Radio Systems," tech. rep., Sept. 2013.

[248] T. Nakamura, S. Abeta, H. Takahashi, and S. Nagata, "Advanced Technologies in LTE/LTE-Advanced," NTT DOCOMO Technical Journal, vol. 15, no. 2, pp. 4-8, 2013.

[249] K. Mallinson, "Mallinson: The 2020 vision for LTE," tech. rep., FierceWirelessEurope.com, 2012.

[250] J. G. Andrews, S. Buzzi, W. Choi, S. Hanly, A. Lozano, A. C. Soong, and J. C. Zhang, "What will 5G be?," IEEE J. Sel. Areas Commun., vol. 32, p. 1065, Jun. 2014.

[251] D. Worth, "V3mobility: University of Surrey achieves 5G speeds of 1Tbps," tech. rep., Feb. 2015.

[252] L. Greenemeier, "Will Millimeter Waves Maximize 5G Wireless?," Scientific American, Jun. 2015. 
[253] S. Deng, M. K. Samimi, and T. S. Rappaport, "28 GHz and 73 GHz MillimeterWave Indoor Propagation Measurements and Path Loss Models," in Proc. IEEE Conf. Commun. Workshop (ICCW), pp. 1244-1250, Jun. 2015.

[254] J. Hoydis, S. Ten Brink, and M. Debbah, "Massive MIMO: How Many Antennas do we need?," in Proc. IEEE Allerton Conf. Commun., pp. 545-550, Sep. 2011.

[255] W. Sievers, "Carriers Taking an Inside Look at LTE coverage," Wireless Week Magazine, Jan. 2016.

[256] T. Parker, "Hurricane Sandy exposed flaw in public-safety LTE plan," tech. rep., Dec. 2012.

[257] Ericsson, "LTE Licensed Assisted Access," tech. rep., Jan. 2015.

[258] Cisco, "Public Safety, Justice, and the Internet of Everything," tech. rep., 2014.

[259] Wind River, "Security in the Internet of Things," tech. rep., 2015.

[260] International Telecommunication Union (ITU), "Provisional Final Acts," World Radiocommunication Conference (WRC-15), pp. 1-452, Nov. 2015.

[261] UK Home Office, "Emergency Service Network," Dec. 2015.

[262] U.K. Home Office, "U.K. Preps for Broadband Mission-Critical Network," MissionCritical Communications magazine, Aug. 2013.

[263] Business Wire, "Calgary Police on Front Line of Public Safety Innovation," Business Wire, Oct. 2015. 
[264] Huawei Technologies Co. Ltd, "eLTE Global Cases Briefing," eLTE Ensuring Mission Critical Smartly, Critical Communications World (CCW), May 2014.

[265] P. Agrawal, J.-H. Yeh, J.-C. Chen, and T. Zhang, "IP Multimedia Subsystems in 3GPP and 3GPP2: Overview and Scalability Issues," IEEE Commun. Mag., vol. 46, pp. 138-145, Jan. 2008.

[266] M. L. Rocca, "RSRQ to SINR," tech. rep., Apr. 2015.

[267] "When to expect mission-critical voice services with LTE networks?," tech. rep., Alcatel-Lucent, Apr. 2014.

[268] Wikipedia, "SDR wiki," tech. rep.

[269] A. Kumbhar, "Overview of ISM bands and Software-defined Radio Experimentation," Wireless Personal Communications, vol. 97, no. 3, pp. 3743-3756, 2017.

[270] F. J. Harris, "On the use of Windows for Harmonic Analysis with the Discrete Fourier Transform," Proc. IEEE, vol. 66, pp. 51-83, Jan. 1978.

[271] R. K. Ganti, F. Baccelli, and J. G. Andrews, "A new way of computing rate in cellular networks," in Proc. IEEE Int. Conf. Commun. (ICC), (Kyoto, Japan), pp. 1-5, June 2011.

[272] S. Newman, J. M. Peha, and J. A. Manner, "The FCC plan for a public safety broadband wireless network," 2010.

[273] M. Tsai, "Path-loss and shadowing (large-scale fading)," tech. rep., National Taiwan University, Oct. 2011.

[274] Xiro Online, "Okumura-Hata," tech. rep., 2017. 
[275] S. Ranvier, "Path loss models," tech. rep., Helsinki University of Technology, Nov. 2004.

[276] M. S. Mollel and M. Kisangiri, "Comparison of Empirical Propagation Path Loss Models for Mobile Communication," Comp. Eng. Intelligent Sys., vol. 5, pp. $1-10,2014$.

[277] A. F. Molisch, Wireless communications, vol. 34. John Wiley \& Sons, 2012.

[278] Y. A. Alqudah, B. Sababha, A. Elnashar, and S. H. Sababha, "On the validation of path loss models based on field measurements using $800 \mathrm{mhz}$ lte network," in Proc. IEEE Annual Sys. Conf. (SysCon), (Orlando,FL), pp. 1-5, 2016.

[279] H. Holma, A. Toskala, and J. Reunanen, LTE Small Cell Optimization: 3GPP Evolution to Release 13. John Wiley \& Sons, Jan. 2016.

[280] B. Soret and K. I. Pedersen, "Macro transmission power reduction for hetnet co-channel deployments," in Proc. IEEE Global Commun. Conf. (GLOBECOM), (Anaheim, CA), pp. 4126-4130, Dec. 2012.

[281] M. S. Ali, “An Overview on Interference Management in 3GPP LTE-Advanced Heterogeneous Networks," Int. J. Future Generation Commun. Netw., vol. 8, pp. 55-68, June 2015.

[282] C. Liu, M. Ding, C. Ma, Q. Li, Z. Lin, and Y.-C. Liang, "Performance analysis for practical unmanned aerial vehicle networks with LoS/NLoS transmissions," in Proc. IEEE Intl. Conf. Commun. Workshops (ICC Workshops), (Kansas City, MO), pp. 1-6, May 2018. 
[283] 3GPP, "Study on 3D channel model for LTE (Release 12)," Technical Specification Group Radio Access Network 36.873, 3rd Generation Partnership Project (3GPP), Dec. 2017. Version 12.7.0.

[284] A. Kammoun, H. Khanfir, Z. Altman, M. Debbah, and M. Kamoun, "Preliminary results on 3D channel modeling: From theory to standard," IEEE J. Sel. Areas Commun. (JSAC), vol. 32, no. 6, pp. 1219-1229, 2014.

[285] 3GPP, "Study on Enhanced LTE Support for Aerial Vehicles (Release 15)," Technical Specification Group Radio Access Network 36.777, 3rd Generation Partnership Project (3GPP), Dec. 2017. Version 15.0.0.

[286] ITU, "Propagation data and prediction methods required for the design of terrestrial broadband millimetric radio access systems," Technical specification ITU Radiocommunication Assembly P.1410-2, International Telecommunication Union (ITU), Apr. 2003.

[287] W. Khawaja, I. Guvenc, D. Matolak, U.-C. Fiebig, and N. Schneckenberger, "A survey of air-to-ground propagation channel modeling for unmanned aerial vehicles," IEEE Commun. Survey Tuts., vol. 21, pp. 2361-2391, May 2019.

[288] H. Binol, I. Guvenc, E. Bulut, and K. Akkaya, "Hybrid evolutionary search method for complex function optimisation problems," IET Electronics Letters, vol. 54, no. 24, pp. 1377-1379, 2018.

[289] H. Binol, E. Bulut, K. Akkaya, and I. Guvenc, "Time optimal multi-uav path planning for gathering its data from roadside units," in Proc. IEEE 88th Vehicular Technology Conference (VTC-Fall), (Chicago, IL), pp. 1-5, Aug. 2018. 
VITA

ABHAYKUMAR KUMBHAR

2004-2008

B.E., Electronics and Communication

Engineering

KLS Gogte Institute of Technology

Belagavi, India

2009-2011

M.S., Computer Science

Villanova University

Villanova, Pennsylvania

\section{PUBLICATIONS AND PRESENTATIONS}

Kumbhar, A. and Damian, M., (2011) Undirected Connectivity of Sparse Yao Graphs. In Proc. Sigma Xi Research Poster Symposium, Villanova Chapter.

Damian, M. and Kumbhar, A., (2011, June). Undirected connectivity of sparse yao graphs. In Proc. 7th ACM SIGACT/SIGMOBILE International Workshop on Foundations of Mobile Computing (pp. 25-32).

Kumbhar, A., (2011) Investigation of Topologies Induced by Smart Antennas. Master's Thesis Villanova University.

Kumbhar, A. and Güvenç, I., (2015, April). A Comparative Study of Land Mobile Radio and LTE-based Public Safety Communications. In Proc. IEEE SoutheastCon 2015 (pp. 1-8).

Koohifar, F., Kumbhar, A. and Guvenc, I., (2016). Receding horizon multi-UAV cooperative tracking of moving RF source. IEEE Communications Letters, 21(6), pp.1433-1436.

Kumbhar, A., Koohifar, F., Güvenç, I. and Mueller, B., (2016). A Survey on Legacy and Emerging Technologies for Public Safety Communications. IEEE Communications Surveys \& Tutorials, 19(1), pp.97-124.

Merwaday, A., Tuncer, A., Kumbhar, A. and Guvenc, I., (2016). Improved throughput coverage in natural disasters: Unmanned aerial base stations for public-safety 
communications. IEEE Vehicular Technology Magazine, 11(4), pp.53-60.

Acuna, V., Kumbhar, A., Vattapparamban, E., Rajabli, F. and Guvenc, I., (2017, March). Localization of wif devices using probe requests captured at unmanned aerial vehicles. In Proc. IEEE Wireless Communications and Networking Conference (WCNC) (pp. 1-6).

Kumbhar, A., Singh, S. and Guvenc, I., (2017, October). UAV assisted public safety communications with LTE-Advanced HetNets and FeICIC. In Proc. IEEE 28th Annual International Symposium on Personal, Indoor, and Mobile Radio Communications (PIMRC) (pp. 1-7).

Kumbhar, A., (2017). Overview of ISM bands and Software-defined Radio Experimentation. Wireless Personal Communications, 97(3), pp.3743-3756.

Kumbhar, A., Güvenç, I., Singh, S. and Tuncer, A., (2017). Exploiting LTEAdvanced HetNets and FeICIC for UAV-assisted public safety communications. IEEE Access, 6, pp.783-796.

Singh, S., Kumbhar, A., Güvenç, I. and Sichitiu, M.L., (2018, August). Distributed Approaches for Inter-Cell Interference Coordination in UAV-Based LTE-Advanced HetNets. In Proc. IEEE 88th Vehicular Technology Conference (VTC-Fall) (pp. $1-6)$.

Kumbhar, A., Binol, H., Guvenç, İ. and Akkaya, K., (2019, January). Interference Coordination for Aerial and Terrestrial Nodes in Three-Tier LTE-Advanced HetNet. In Proc. IEEE Radio and Wireless Symposium (RWS) (pp. 1-4).

Dambal, V.A., Mohadikar, S., Kumbhar, A. and Guvenc, I., (2019, March). Improving LoRa Signal Coverage in Urban and Sub-Urban Environments with UAVs. In Proc. International Workshop on Antenna Technology (iWAT) (pp. 210-213).

Kumbhar, A., Binol, H., Singh, S., Guvenç, İ. and Akkaya, K., (2019). Heuristic Approach for Jointly Optimizing FeICIC and UAV Locations in Multi-Tier LTEAdvanced Public Safety HetNet. Submitted IET Cyber-systems and Robotics. 